\title{
A COMPILATION OF DATA ON SOME SPECTRA \\ OF THORIUM
}

G. W. Charles

DATE ISSUED

MAY 22, 1958

OAK RIDGE NATIONAL LABORATORY

Oak Ridge, Tennesseo

operated by

UNION CARBIDE CORPORATION

for the

U.S. ATOMIC ENERGY COMMISSION 


\section{DISCLAIMER}

This report was prepared as an account of work sponsored by an agency of the United States Government. Neither the United States Government nor any agency Thereof, nor any of their employees, makes any warranty, express or implied, or assumes any legal liability or responsibility for the accuracy, completeness, or usefulness of any information, apparatus, product, or process disclosed, or represents that its use would not infringe privately owned rights. Reference herein to any specific commercial product, process, or service by trade name, trademark, manufacturer, or otherwise does not necessarily constitute or imply its endorsement, recommendation, or favoring by the United States Government or any agency thereof. The views and opinions of authors expressed herein do not necessarily state or reflect those of the United States Government or any agency thereof. 


\section{DISCLAIMER}

Portions of this document may be illegible in electronic image products. Images are produced from the best available original document. 


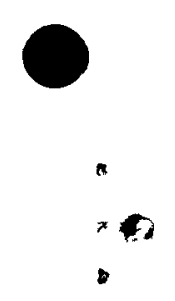

$n$ 


\section{CONTENTS}

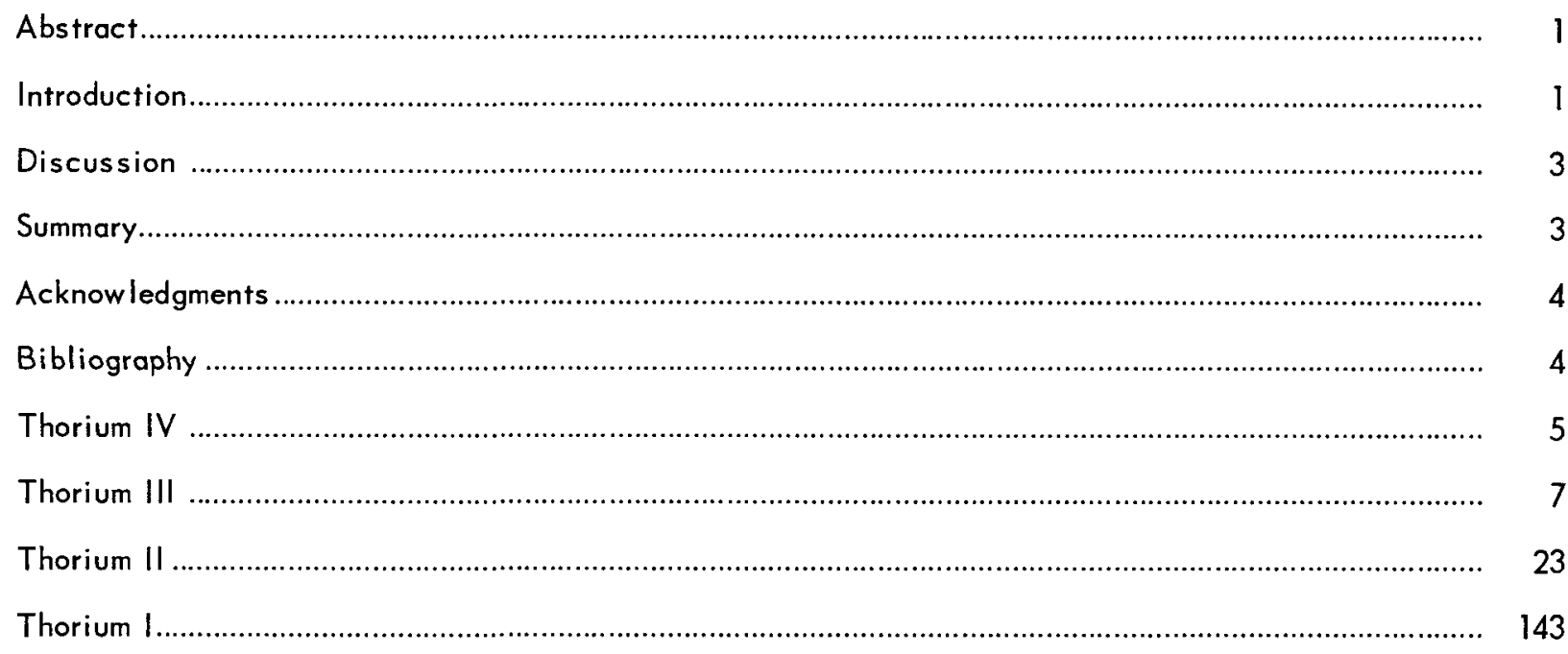

LIST OF TABLES

Table 1. Energy Levels of Th IV ................................................................................................

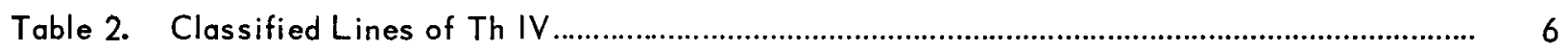

Table 3. Energy Levels of Th III ................................................................................................ 8

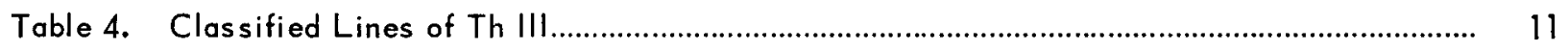

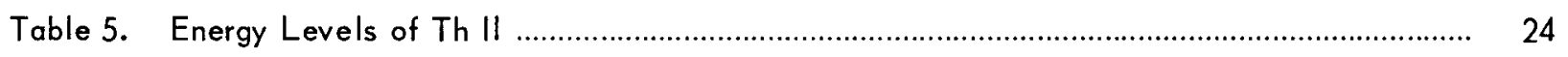

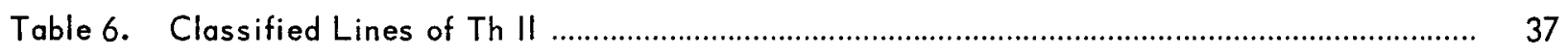

Table 7. Zeeman Data of Unclassified Th II Lines ............................................................................ 138

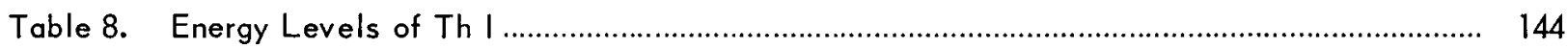

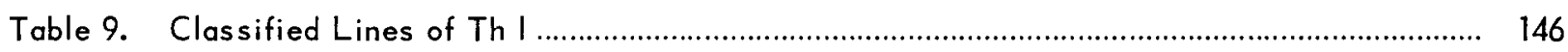

Table 10. Zeeman Data of Unclassified Th I Lines .................................................................... 151

Table 11. I sotope Shift Data of Unclassified Lines .......................................................................... 153

Table 12. Unclassified Furnace Lines of Thorium .................................................................... 155 


\title{
A COMPILATION OF DATA ON SOME SPECTRA OF THORIUM
}

\author{
G. W. Charles \\ Physics Division
}

\begin{abstract}
A compilation of available data on the spectra of thorium is presented, including energy levels and classified lines with Zeeman effects and isotope shifts, as well as certain data on unclassified lines. The present status of knowledge of these spectra is discussed.
\end{abstract}

\section{INT RODUCTION}

Over a period of many years, much information has been amassed on the spectra of thorium. Most of this information has been published. It is the purpose of this report to present a compilation of the available data on thorium spectra exclusive of a list of unclassified lines. ${ }^{1}$ The spectra will be considered in the order Th IV, III, II, and I.

\section{Th iv}

The first classifications in this one-electron spectrum were made by Lang (1). Lier reported Zeeman observations on one line in his dissertation (2); additional Zeeman work was done by de Bruin and Klinkenberg (3). Improved measurements and a more complete description were reported by Klinkenberg and Lang (4) and by Klinkenberg (5). Table 1 gives the known energy levels of Th IV in the form that has been adopted for the National Bureau of Standards compilation contained in NBS Circular 467 . In the references to this and the similar tables (Tables 3,5, and 8) the following notation is employed:

$$
\begin{aligned}
T & =\text { term, } \\
C L & =\text { classified lines, } \\
Z E & =\text { Zeeman effect observations, } \\
G D & =\text { Grotrian diagram, } \\
I S & =\text { isotope shift data, } \\
T h & =\text { theory, } \\
I P & =\text { ionization potential data. }
\end{aligned}
$$

\footnotetext{
${ }^{2}$ No list of unclassified lines has been included, partly because of considerations of space and partly because the list of $C$. C. Kiess of the National Bureau of Standards is more comprehensive.
}

Table 2 lists the classified lines of Th IV, taken from the reports of Klinkenberg and Lang (4) and Klinkenberg (5). The observed $g$ values in Table 1 have been estimated by the author from published Zeeman data $(4,5)$. In Table 2, an asterisk in the wave length column indicates that the wave length was measured at the Massachusetts Institute of Technology (MIT). $\Delta \sigma_{\text {vac }}$ is the difference between observed frequency and calculated frequency. In the column labelled "Zeeman patterns," the complete observed patterns are listed, with figures in parentheses giving $\pi$ components and the other figures giving $\sigma$ components (measured in Lorentz units - 1 Lorentz unit being $4.669 \times 10^{-5}$ times the magnetic field strength, $H$, in oersteds). When more than one component of a given kind is listed, the strongest component is underlined.

\section{Th III}

The earliest work on this spectrum was also reported by Lang (6). Zeeman effects of several lines were reported by Lier (2). Extensions of the analysis were reported by de Bruin and Klinkenberg (3) and de Bruin, Klinkenberg, and Schuurmans (7). Later, Klinkenberg reported a revised and extended analysis (8). Racah has compared the experimental energy levels of Klinkenberg with the predictions of theory (9). The energy levels of Th 111, taken from Klinkenberg (8), are listed in Table 3. The quantity $X$ has not been definitely established; Klinkenberg has calculated it to be $810 \mathrm{~cm}^{-1}$ from two intercombination lines (8), while Racah has stated that it must be small (9).

Table 4 lists the classified lines of Th III, taken from the report of Klinkenberg (5) except for 
some lines for which wave lengths and Zeeman data are taken from the MIT list. The complete observed Zeeman patterns are listed and the strongest components are underlined. The edge of an unresolved group of $\pi$ components is denoted by $E_{\pi}$, while $G_{\sigma}$ denotes the centroid of an unresolved $\sigma$ group.

\section{Th II}

This spectrum has been analyzed by McNally, Harrison, and Park (10) and by de Bruin, Schuurmans, and Klinkenberg $(11,12)$. Earlier work was done by Lier (2). Two sets of energy levels were found with no connection established between them. The connection between the two sets of levels was established by McNally in a study of the infrared spectrum of thorium (13). This confirmed the preliminary results of de Bruin et al. (12). A theoretical study of the low, even configurations $6 d^{2} 7 s, 6 d 7 s^{2}$, and $6 d^{3}$ was made by Eisenberg (14). A study of the isotope shift between $\mathrm{Th}^{232}$ and $\mathrm{Th}^{230}$ lines was carried out by Stukenbroeker and McNally (15).

The energy levels of Th 11 are listed in Table 5. The assignment of levels to the configurations $6 d^{2} 7 s, 6 d 7 s^{2}$, and $6 d^{3}$ is confirmed by the theoretical study of Eisenberg (14), while the assignment of levels to the configurations $5 f 7 s^{2}$ and $5 f 6 d 7 s$ is confirmed by unpublished theoretical work of Racah. Other levels and configuration assignments are taken from the papers of McNally (13) and of de Bruin et al. $(11,12)$. The remainder of the configuration assignments are tentative suggestions of the author. Table 5 contains 45 energy levels not previously published. These have been communicated to the author by Schuurmans and Klinkenberg and by Klinkenberg. It al so contains 43 previously unpublished $g$ values, of which 24 were determined by the author. In addition, $g$ values have been improved for 20 levels by the author from unpublished MIT Zeeman data.

The elassified lines of Th 11 are listed in Table 6. The total number of lines listed here is more than 2850, compared with 1091 lines listed by MeNally, Harrison, and Park (10) in the only published list of classified lines of Th 11 , and with 1931 lines in an unpublished list of Klinkenberg. The data for Table 6 were obtained in the following manner. A list of all known energy levels of Th 11 was submitted to the IBM group at the Oak Ridge Gaseous Diffusion Plant. This list contained 165 odd and 191 even levels. Wave lengths of approximately 9600 allowed lines of Th 11 were calculated from the levels. This list of lines was compared with a list of observed lines that included ones taken from the MIT card file on thorium (symbol $M$ ), from three University of Chicago MS theses $(16,17,18)$ (symbol C), from the paper of McNally (13) (symbol Mc), from the Vatican wave length data (19) (symbol V), from Klinkenberg's list of unpublished Th II classified lines (symbol K), and a few from Schuurmans' dissertation (20) (symbol S). All coincidences between the two lists within $0.2 \mathrm{~cm}^{-1}$ were recorded.

As in Table 2, $\Delta \sigma_{\text {vac }}$ signifies the difference between observed frequency and frequency calculated from the energy levels. In order to save space, the shorthand notation taken from the paper of McNally, Harrison, and Park (10) for the description of Zeeman patterns has been used in Table 6. The data given are type of pattern, strongest $\pi$ component (in parentheses), strongest $\sigma$ component, and $g$ values of the two levels in the same order in which the levels appear in the transition column. In the interest of uniformity and simplicity, the odd level is stated first, followed by the even level. Three types of patterns are distinguished by the numbers 4,5 , and 6 . Types 4 and 5 are $J_{1} \neq J_{2}$, shade out and shade in, respectively, while Type 6 is $J_{1} \neq J_{2}$, symmetrical. Zeeman data are taken from the paper of McNally, Harrison, and Park and from unpublished MIT data (symbol M), from Klinkenberg's list (symbol K), and from Lier's dissertation (symbol $L$ ). A $g$ value given in parentheses was assumed because the Zeeman data for the line were insufficient to calculate $g$ values for both levels.

Table 7 gives Zeeman effect data for some unclassified lines of Th 11 taken from the MIT unpubli shed data.

\section{Th I}

The spectrum of Th I is by far the most poorly analyzed of all the thorium spectra. The only published works are Schuurmans' dissertation (20), which lists some energy levels, and Lier's dissertation (2), which gives some Zeeman data. The known energy levels are given in Table 8, which is taken from Schuurmans' dissertation and from an unpublished list of McNally. Where a $g$ value is listed by McNally, it has been adopted. Data taken from Schuurmans are indicated by an (S). 
Table 9 lists the classified lines of Th I. A list was calculated by the author from the energy levels and then compared with the data. The reference symbols are the same as those used in Table 6. Under Zeeman data, the three types here are designated 1,2, and 3. Type 1 and Type 2 are $J_{1} \neq$ $J_{2}$, shade out and shade in, respectively, while Type 3 is $J_{1}=J_{2}$, symmetrical. Table 10 gives Zeeman data for several unclassified lines of integral $J$, which are mostly Th 1 .

\section{Miscellaneous Data}

In Table 11 are collected isotope shift data of unclassified thorium lines, consisting of the unpublished data of Stukenbroeker and McNally. An attempt has been made to identify the emitter of the line as either Th I or Th II. The intensities are from the MIT list. Table 12 lists the remaining unclassified lines in the furnace spectrum. It will be observed that most of the se appear to be Th 1. The furnace spectrum is contained in Fred's list (21). Table 13 contains the unclassified absorption lines. The absorption spectrum, observed with the under-water spark and with the exploding wire, was reported by de Bruin and Lier (22). Five degrees of absorption were distinguished. Those lines showing the heaviest absorption are designated by the numeral 1 in Table 13. Again the intensities are from the MIT list. The notation "Zeeman Effect" in the column labelled "Probable Spectrum" signifies that Zeeman data are available on the line. An asterisk is placed in this column to denote that the line has been classified, but that the absorption seems too strong to be consistent with the classification.

\section{DISCUSSION}

An evaluation of the state of knowledge of the thorium spectra follows. Every line that has been ascribed to Th IV has been classified. Any extension of this spectrum therefore depends on improved observations, which is likely to be a fairly difficult task.

The energy level scheme of Th III is quite complete in so far as first series members are concerned. The agreement between theory and observation with regard to relative positions of levels and to $g$ values is very good. The principal lines of endeavor to improve knowledge of this spectrum would seem to be the definite establish. ment of the connection between the two sets of energy levels and the finding of higher series members. All the lines that can be ascribed to Th 111 are listed in the paper by Klinkenberg (5); not many of them remain unclassified. An extension of the Th III spectrum would al so require new observations.

Although there are about 350 energy levels of Th II known; knowledge of this spectrum is not complete. Theoretical studies are needed to establish the configurations of energy levels already known. In addition, there are many remaining unclassified lines of Th II and undoubtedly many more could be observed under suitable conditions.

Compared with the remainder of the thorium spectra, that of Th 1 is practically unknown, although it is expected to be the most complex of all. This is probably due to the difficulty of exciting this spectrum in the arc. More work is needed on every phase of this spectrum. A more intensive study of the furnace spectrum, especially at wave lengths greater than $5000 \AA$, should be profitable.

\section{SUMMARY}

A compilation of data on the known spectra of thorium is presented. Included are energy levels and classified lines with Zeeman effects and isotope shifts for Th IV, Th III, Th II, and Th I. Data are also included on unclassified lines embracing Zeeman effects, isotope shifts, appearance in furnace spectrum, and appearance in absorption spectrum. The principal results of this investigation are:

1. the assembling in one document of all the available data on classified thorium spectra,

2. a significant extension of the knowledge of $g$ values of Th II,

3. the collection of some significant data for a further extension of classification of the thorium spectra.

The status of knowledge of each of these spectro is discussed. It is concluded that $T h$ IV and Th III can only be extended by new observations; both these spectra are found to be in reasonably satisfactory condition. Some extension of Th II can be made with presently available data. A theoretical study is needed on configuration assignments of known Th II levels. The knowledge of Th $\mathrm{l}$ is concluded to be in a very unsatisfactory condition, with more work needed on all phases of this spectrum. 


\section{ACKNOWLEDGMENTS}

The author is indebted to all who have made available unpublished data used in this compilation. These include G. R. Harrison, Dean of Science at MIT; for wave length data and Zeeman measurements, P. F. A. Klinkenberg for lists of classified Th II lines; P. F. A. Klinkenberg and Ph. Schuurmans for Th 11 energy levels; G. Racah for the results on energy levels from the configura- tions $5 f 7 s^{2}$ and $5 f 6 d 7 s$; and J. R. McNally for the Th I levels and isotope shift data. The assistance of $B$. Carter of the IBM group at the Oak Ridge Gaseous Diffusion Plant with the calculations of Th 11 wave lengths is gratefully acknowledged. Special thanks are due to J. R. McNally, Jr., who initiated this project, for suggesting it to the author, and for his constant interest and encouragement.

\section{BIBLIOGRAPHY}

1. R. J. Lang, Can. J. Research A14, 43 (1936).

2. J. N. Lier, Doctoral Dissertation, Amsterdam (1939).

3. T. L. de Bruin and P. F. A. Klinkenberg, Proc. Acad. Sci. Amsterdam 43, 581 (1940).

4. P. F. A. Klinkenberg and R. J. Lang, Physica 15, 774 (1949).

5. P. F. A. Klinkenberg, Pbysica 16, 185 (1950).

6. R. J. Lang, Phys. Rev. 56, 272 (1939).

7. T. L. de Bruin, P. F. A. Klinkenberg, and Ph. Schuurmans, Z. Physik. 118, 58 (1941).

8. P. F. A. Klinkenberg, Physica 16, 618 (1950).

9. G. Racah, Physica 16, 651 (1950).

10. J. R. McNally, Jr., G. R. Harri son, and H. B. Park, J. Opt. Soc. Am. 32, 334 (1942).

11. T. L. de Bruin, Ph. Schuurmans, and P. F. A. Klinkenberg, Z. Physik. 121, 667 (1943).

12. T. L. de Bruin, P. F. A. Klinkenberg, and Ph. Schuurmans, Z. Physik. 122, 23 (1943).

13. J. R. McNally, Jr., J. Opt. Soc. Am. 35, 390 (1945).

14. Y. Ei senberg, Pbysica 18, 177 (1952).

15. G. L. Stukenbroeker and J. R. McNally, Jr., J. Opt. Soc. Am. 43, 36 (1953).

16. M. Fred, MS Thesis, University of Chicago (1934).

17. M. Ference, Jr., MS Thesis, University of Chicago (1934).

18. R. B. Bowersox, MS Thesis, University of Chicago (1934).

19. A. Gatterer and J. Junkes, Spektren der Seltenen Erden, Specola Vaticana, Vatican City, 1945, p 329347.

20. Ph. Schuurmans, Doctoral Dissertation, Amsterdam (1946).

21. M. Fred, Astrophys. J. 87, 176 (1938).

22. T. L. de Bruin and J. N. Lier, Proc. Acad. Sci. Amsterdam 41, 956 (1938). 


\section{THORIUM}

Th IV

87 Electrons

Ground State: $1 s^{2} 2 s^{2} 2 p^{6} 3 s^{2} 3 p^{6} 3 d^{10} 4 s^{2} 4 p^{6} 4 d^{10} 4 f^{14} 5 s^{2} 5 p^{6} 5 d^{10} 6 s^{2} 6 p^{6} 5 f^{2} F_{21 / 2}^{\circ}$

$5 f^{2} \mathrm{~F}_{21 / 2}^{\circ}=231900 \mathrm{~cm}^{-1}$

I. P. $=28.6$ volts

Twelve levels have been given by Klinkenberg and Lang, extending and revising the level scheme originally proposed by Lang and confirmed in part by the Zeeman effect of $7^{2} S_{1 / 2}-7^{2} P_{1 / 2}$ observed by Lier. The major correction pertained to the position of $5^{2} \mathrm{~F}$ which was found to be the ground state. The observed $g$ values listed in the table have been calculated by $G$. W. Charles from published data on Zeeman patterns.

\section{REFERENCES}

R. J. Lang, Can. J. Res. A 14, 43 (1936). (CL) (T)

J. N. Lier, Doctoral Dissertation, Amsterdam (1939). (ZE)

T. L. De Bruin and P. F. A. Klinkenberg, Proc. Acad. Sci. Amsterdam 43, 581 (1940). (ZE)

P. F. A. Klinkenberg and R. J. Long, Pbysica 15, 774 (1949). (CL) (T) (GD) (ZE) (IP)

P. F. A. Klinkenberg, Physica 16, 185 (1950). (T) (CL) (ZE) (GD)

Table 1. Energy Levels of Th IV

\begin{tabular}{|c|c|c|c|c|c|}
\hline Configuration & Designation & $J$ & $\begin{array}{l}\text { Level } \\
\left(\mathrm{cm}^{-1}\right)\end{array}$ & $\begin{array}{l}\text { Interval } \\
\left(\mathrm{cm}^{-1}\right)\end{array}$ & Observed $g$ Value \\
\hline $5 f$ & $5 f^{2} \mathrm{~F}^{\circ}$ & $\begin{array}{l}21 / 2 \\
31 / 2\end{array}$ & $\begin{array}{r}0.00 \\
4325.74\end{array}$ & 4325.74 & $0.80 ?$ \\
\hline $6 d$ & $6 d^{2} \mathrm{D}$ & $\begin{array}{l}11 / 2 \\
21 / 2\end{array}$ & $\begin{array}{r}9192.84 \\
14486.32\end{array}$ & 5293.48 & $1.14 ?$ \\
\hline $7 s$ & $7 s^{2} s$ & $1 / 2$ & 23130.3 & & 2.024 \\
\hline $7 p$ & $7 p^{2} \mathrm{P}^{\circ}$ & $\begin{array}{r}1 / 2 \\
11 / 2\end{array}$ & $\begin{array}{l}60238.9 \\
73055.6\end{array}$ & 12816.7 & 0.666 \\
\hline $8 s$ & $8 s^{2} S$ & $1 / 2$ & 119621.2 & & 2.03 \\
\hline $7 d$ & $7 d^{2} \mathrm{D}$ & $\begin{array}{l}11 / 2 \\
21 / 2\end{array}$ & $\begin{array}{l}119684.3 \\
121426.6\end{array}$ & 1742.3 & $\begin{array}{l}0.81 \\
1.23\end{array}$ \\
\hline $8 p$ & $8 p^{2} p^{0}$ & $\begin{array}{l}1 / 2 \\
11 / 2\end{array}$ & $\begin{array}{l}134516.1 \\
139870.4\end{array}$ & 5354.3 & $\begin{array}{l}0.66 \\
1.33\end{array}$ \\
\hline
\end{tabular}


Table 2. Classified Lines of Th IV

\begin{tabular}{|c|c|c|c|c|}
\hline$\lambda(\stackrel{\circ}{\AA})$ & $\begin{array}{c}\sigma_{\text {vac }} \\
\left(\mathrm{cm}^{-1}\right)\end{array}$ & $\begin{array}{l}\Delta \sigma_{\mathrm{vac}} \\
\left(\mathrm{cm}^{-1}\right)\end{array}$ & Classification & Observed Zeeman Pattern \\
\hline 10875.05 & 9192.8 & 0.0 & $5^{2} F_{21 / 2}^{O}-6{ }^{2} D_{1 / 2}$ & \\
\hline 9839.25 & 10160.59 & +0.01 & $5^{2} F_{31 / 2}^{\circ}-6^{2} D_{21 / 2}$ & \\
\hline 6901.16 & 14486.33 & +0.01 & $5^{2} \mathrm{~F}_{21 / 2}^{\mathrm{O}}-6^{2} \mathrm{D}_{21 / 2}$ & $(0.47)(\underline{0.85}) 0.60, \underline{0.98}, 1.34$ \\
\hline 6740.37 & 14831.89 & +0.1 & $7^{2} D_{1 / 2}-8^{2} P_{1 / 2}^{\circ}$ & (0) 0.87 \\
\hline 6711.87 & 14894.87 & 0.0 & $8^{2} S_{1 / 2}-8{ }^{2} P_{1 / 2}^{\circ}$ & $(0.70) 1.36$ \\
\hline 5420.38 & 18443.78 & 0.0 & $7^{2} \mathrm{D}_{2 y_{2}}-8^{2} \mathrm{P}_{1 / 2}^{\circ}$ & (0) 1.08 \\
\hline 4952.52 & 20186.12 & 0.0 & $7^{2} \mathrm{D}_{1 / 1 / 2}-8^{2} \mathrm{P}_{1 / 2}^{\circ}$ & $(0.79) 0.90$ \\
\hline 4937.09 & 20249.21 & 0.0 & $8^{2} S_{1 / 2}-8{ }^{2} P_{1 / 2}^{0}$ & $(0.34) 1.01,1.67$ \\
\hline $2693.995^{\star}$ & 37108.57 & 0.0 & $7^{2} \mathrm{~S}_{1 / 2}-7^{2} \mathrm{P}_{1 / 2}^{\circ}$ & $(0.679) 1.345^{*}$ \\
\hline 2296.81 & 43525.2 & & $6^{2} \mathrm{~F}_{3 Y_{2}}^{\circ}-8^{2} \mathrm{D}_{2 Y_{2}}{ }^{2}$ & \\
\hline 2261.26 & 44209.4 & & $6^{2} F_{21 / 2}^{\circ}-8^{2} D_{1 / 2} ?$ & \\
\hline 2242.11 & 44586.9 & & $6^{2} F_{21 / 2}^{O}-8^{2} D_{21 / 2} ?$ & \\
\hline 2146.81 & 46566.1 & +0.5 & $7^{2} P_{1 / 2}^{\circ}-8{ }^{2} S_{1 / 2}$ & \\
\hline 2143.91 & 46629.1 & +0.4 & $7^{2} \mathrm{P}_{1 / 2}^{\circ}-7^{2} \mathrm{D}_{1 / 2}$ & \\
\hline 2066.70 & 48371.0 & 0.0 & $7^{2} \mathrm{P}_{1 / / 2}^{\circ}-7^{2} \mathrm{D}_{21 / 2}$ & \\
\hline 2002.34 & 49925.3 & 0.0 & $7^{2} S_{1 / 2}-7^{2} P_{1 / 2}^{0}$ & \\
\hline $1959.02^{\star *}$ & 51045.9 & -0.2 & $6^{2} D_{1 / 2}-7{ }^{2} P_{y_{2}}^{\circ}$ & \\
\hline 1707.37 & 58569.7 & -0.4 & $6^{2} \mathrm{D}_{21 / 2}-7^{2} \mathrm{P}_{11 / 2}^{\circ}$ & \\
\hline 1684.01 & 59382.0 & -0.3 & $7^{2} \mathrm{P}_{1 / 2}^{\circ}-8^{2} \mathrm{~S}_{1 / 2}$ & \\
\hline 1682.22 & 59445.3 & -0.1 & $7^{2} \mathrm{P}_{1 / 2}^{\circ}-7^{2} \mathrm{D}_{1 / 2}$ & \\
\hline 1565.85 & 63863.0 & +0.2 & $6^{2} \mathrm{D}_{11 / 2}-7^{2} \mathrm{P}_{11 / 2}^{0}$ & \\
\hline 854.02 & 117093 & -7.9 & $5^{2} \mathrm{~F}_{31 / 2}^{\circ}-7^{2} \mathrm{D}_{21 / 2} ?$ & \\
\hline 835.55 & 119682 & -2.3 & $5^{2} \mathrm{~F}_{21 / 2}^{\circ}-7^{2} \mathrm{D}_{1 / 2}$ & \\
\hline 797.53 & 125387 & +2.9 & $6^{2} \mathrm{D}_{2 y_{2}}-8^{2} \mathrm{P}_{1 / 2}^{\circ}$ & \\
\hline
\end{tabular}

*MIT measurement. Other data are from Klınkenberg and Lang (1949) and from Klınkenberg (1950).

* Wave lengths above $2000 \mathrm{~A}$ are in air, while those below $2000 \mathrm{~A}$ are in vacuo. 


\section{THORIUM}

\section{Th III}

88 Electrons

$Z=90$

Ground State: $1 s^{2} 2 s^{2} 2 p^{6} 3 s^{2} 3 p^{6} 3 d^{10} 4 s^{2} 4 p^{6} 4 d^{10} 4 f^{14} 5 s^{2} 5 p^{6} 5 d^{10} 6 s^{2} 6 p^{6} 6 d^{2}{ }^{3} \mathrm{~F}_{2}$ $6 d^{2}{ }^{3} F_{2}=161000 \mathrm{~cm}^{-1}$

I. $P_{.}=19.9$ volts

The energy levels are taken from the paper by Klinkenberg, who has revised and extended earlier analyses. Two groups of levels are known. The quantity $X$ is found to be $810 \mathrm{~cm}^{-1}$ from two intercombination lines, but this has not been definitely established. The ionization potential is provisionally taken to be 19.9 volts. An obvious typographical error in the original paper in the value of $6 d 7 s^{3} D_{1}$ has been corrected.

\section{REFERENCES}

R. J. Lang, Phys. Rev. 56, 272 (1939). (T) (CL)

J. N. Lier, Doctoral Dissertation, Amsterdam (1939). (ZE)

T. L. de Bruin and P. F. A. Klinkenberg, Proc. Acad. Sct. Amsterdam 43, 581 (1940). (T) (CL) (ZE)

T. L. de Bruin, P. F. A. Klinkenberg, and Ph. Schuurmans, Z. Physik. 118, 58 (1941). (T) (CL) (ZE)

P. F. A. Klinkenberg, Pbystca 16, 618 (1950). (T) (CL) (ZE) (GD) (IP)

G. Racah, Pbysica 16, 651 (1950). 
Table 3. Energy Levels of Th III

\begin{tabular}{|c|c|c|c|c|c|}
\hline Configuration & Designation & $J$ & $\begin{array}{l}\text { Level } \\
\left(\mathrm{cm}^{-1}\right)\end{array}$ & $\begin{array}{l}\text { Interval } \\
\left(\mathrm{cm}^{-1}\right)\end{array}$ & Observed $g$ Value \\
\hline \multicolumn{6}{|c|}{ Group B } \\
\hline \multirow[t]{3}{*}{$6 d^{2}$} & $6 d^{3} \mathrm{~F}$ & 2 & 0.0 & \multirow{3}{*}{$\begin{array}{l}3992.7 \\
2482.2\end{array}$} & 0.744 \\
\hline & & 3 & 3992.7 & & 1.083 \\
\hline & & 4 & 6474.9 & & $1.20_{5}$ \\
\hline $6 d^{2}$ & $6 d^{l} \mathrm{D}$ & 2 & 4613.3 & & 1.020 \\
\hline \multirow[t]{3}{*}{$6 d^{2}$} & $6 d^{3} \mathrm{P}$ & 0 & 5027.0 & \multirow{3}{*}{$\begin{array}{l}2785.5 \\
2564.6\end{array}$} & \\
\hline & & 1 & 7812.5 & & $1.50_{5}$ \\
\hline & & 2 & 10377.1 & & 1.367 \\
\hline \multirow[t]{3}{*}{$6 d 7 s$} & $7 s^{3} \mathrm{D}$ & 1 & 5460.8 & \multirow{3}{*}{1652.2} & $0.50_{5}$ \\
\hline & & 2 & 7113.0 & & 1.180 \\
\hline & & 3 & 9890.6 & & 1.339 \\
\hline $6 d^{2}$ & $6 d^{\mathrm{T}} \mathrm{G}$ & 4 & 10479.3 & & 1.05 \\
\hline $7 s^{2}$ & $7 s^{1} \mathrm{~s}$ & 0 & 11897.9 & & \\
\hline $6 d 7 s$ & $7 s^{1} \mathrm{D}$ & 2 & 15974.4 & & $1.00_{0}$ \\
\hline $6 d^{2}$ & $6 d^{1} \mathrm{~S}$ & 0 & 18929.6 & & \\
\hline \multirow[t]{2}{*}{$6 d 7 p$} & $7 p(3 / 2,1 / 2)^{\circ}$ & 1 & 39217.7 & \multirow[b]{2}{*}{-2000.5} & 0.900 \\
\hline & & 2 & 37217.2 & & 0.795 \\
\hline \multirow[t]{3}{*}{$7 s 7 p$} & $7 p^{3} p^{0}$ & 0 & 42196.3 & \multirow{3}{*}{$\begin{array}{l}2804.4 \\
8087.6\end{array}$} & \\
\hline & & 1 & 45000.7 & & 1.120 \\
\hline & & 2 & 53088.3 & & 1.36 \\
\hline \multirow[t]{2}{*}{$6 d 7 p$} & $7 p(5 / 2,1 / 2)^{\circ}$ & 2 & 44024.9 & \multirow[b]{2}{*}{377.1} & 1.200 \\
\hline & & 3 & 44402.0 & & 1.125 \\
\hline \multirow[t]{4}{*}{$6 d 7 p$} & $7 p(3 / 2,3 / 2)^{\circ}$ & 0 & 51682.1 & \multirow{4}{*}{$\begin{array}{r}-752.8 \\
-3312.6 \\
2300.7\end{array}$} & \\
\hline & & 1 & 50929.3 & & 1.22 \\
\hline & & 2 & 47616.7 & & 1.02 \\
\hline & & 3 & 49917.4 & & 1.19 \\
\hline \multirow[t]{4}{*}{$6 d 7 p$} & $7 p(5 / 2,3 / 2)^{\circ}$ & 1 & 53876.0 & \multirow{4}{*}{$\begin{array}{r}1459.8 \\
153.3 \\
-2499.9\end{array}$} & 1.25 \\
\hline & & 2 & 55335.8 & & $\sim 1.33$ \\
\hline & & 3 & 55489.1 & & 1.10 \\
\hline & & 4 & 52989.2 & & 1.27 \\
\hline \multirow[t]{2}{*}{$7 s 7 p$} & $7 p^{1} \mathrm{P}^{\circ}$ & 1 & 68937.1 & & \\
\hline & \multicolumn{5}{|c|}{ Group A } \\
\hline \multirow[t]{3}{*}{$5 f 6 d$} & $6 d^{3} \mathrm{H}^{\circ}$ & 4 & $0.00+X$ & \multirow{3}{*}{$\begin{array}{l}4489.6 \\
3947.3\end{array}$} & $0.88_{5}$ \\
\hline & & 5 & $4489.56+X$ & & 1.04 \\
\hline & & 6 & $8436.9+X$ & & $1.16_{7}$ \\
\hline
\end{tabular}


Table 3 (continued)

\begin{tabular}{|c|c|c|c|c|c|}
\hline Configuration & Designation & $J$ & $\begin{array}{l}\text { Level } \\
\left(\mathrm{cm}^{-1}\right)\end{array}$ & $\begin{array}{l}\text { Interval } \\
\left(\mathrm{cm}^{-1}\right)\end{array}$ & Observed $g$ Value \\
\hline \multicolumn{6}{|c|}{ Group A (continued) } \\
\hline \multirow[t]{3}{*}{$5 / 6 d$} & $6 d^{3} F^{\circ}$ & 2 & $510.69+X$ & \multirow{3}{*}{$\begin{array}{l}4316.12 \\
4153.74\end{array}$} & 0.739 \\
\hline & & 3 & $4286.81+X$ & & 1.003 \\
\hline & & 4 & $8980.55+X$ & & 1.188 \\
\hline \multirow[t]{3}{*}{$5 / 7 s$} & $7 s^{3} \mathrm{~F}^{\circ}$ & 2 & $3181.40+X$ & \multirow{3}{*}{$\begin{array}{r}-654.38 \\
3783.71\end{array}$} & 0.725 \\
\hline & & 3 & $2527.02+X$ & & 1.071 \\
\hline & & 4 & $6310.73+X$ & & $1.21_{8}$ \\
\hline $5 f 6 d$ & $6 d^{1} G^{\circ}$ & 4 & $3188.26+X$ & & 0.976 \\
\hline \multirow[t]{3}{*}{$5 f 6 d$} & $6 d^{3} G^{\circ}$ & 3 & $5060.62+X$ & \multirow{3}{*}{$\begin{array}{l}3081.02 \\
3135.01\end{array}$} & 0.869 \\
\hline & & 4 & $8141.64+X$ & & 1.103 \\
\hline & & 5 & $11276.65+X$ & & 1.186 \\
\hline $5 / 6 d$ & $6 d^{1} \mathrm{D}^{\circ}$ & 2 & $6288.21+X$ & & 0.908 \\
\hline $5 / 7 s$ & $7 s^{1} F^{\circ}$ & 3 & $7500.48+X$ & & 1.027 \\
\hline \multirow[t]{3}{*}{$5 f 6 d$} & $6 d^{3} D^{\circ}$ & 1 & $7921.01+X$ & \multirow{3}{*}{$\begin{array}{r}2259.8 \\
560.3\end{array}$} & 0.622 \\
\hline & & 2 & $10180.8+X$ & & $1.19_{3}$ \\
\hline & & 3 & $10741.07+X$ & & 1.224 \\
\hline \multirow[t]{3}{*}{$5 f 6 d$} & $6 d^{3} \mathrm{P}^{\circ}$ & 0 & $11232.82+X$ & \multirow{3}{*}{$\begin{array}{r}-109.62 \\
2085.09\end{array}$} & \\
\hline & & 1 & $11123.20+X$ & & 1.352 \\
\hline & & 2 & $13208.29+X$ & & 1.432 \\
\hline $5 / 6 d$ & $6 d^{l} \mathrm{~F}^{\circ}$ & 3 & $15453.44+X$ & & $1.07_{0}$ \\
\hline $5 / 6 d$ & $6 d^{1} H^{\circ}$ & 5 & $19009.83+X$ & & $1.01_{5}$ \\
\hline $5 / 6 d$ & $6 d^{1} \mathrm{P}^{\circ}$ & 1 & $20225.2+X$ & & 1.009 \\
\hline \multirow[t]{3}{*}{$5 f^{2}$} & $5 f^{3} \mathrm{H}$ & 4 & $15148.5+X$ & \multirow{3}{*}{$\begin{array}{l}2739.0 \\
2883.5\end{array}$} & $0.81_{0}$ \\
\hline & & 5 & $17887.5+X$ & & 1.01 \\
\hline & & 6 & $20771.0+X$ & & 1.16 \\
\hline \multirow[t]{3}{*}{$5 f^{2}$} & $5 f^{3} \mathrm{~F}$ & 2 & $18863.82+X$ & \multirow{3}{*}{$\begin{array}{r}1976.66 \\
943.35\end{array}$} & 0.694 \\
\hline & & 3 & $20840.48+X$ & & 1.096 \\
\hline & & 4 & $21783.83+X$ & & $1.18_{0}$ \\
\hline $5 f^{2}$ & $5 f^{1} \mathrm{G}$ & 4 & $25972.12+X$ & & 1.072 \\
\hline $5 f^{2}$ & $5 f^{7} \mathrm{D}$ & 2 & $28232.42+X$ & & 1.126 \\
\hline \multirow[t]{3}{*}{$5 f^{2}$} & $5 f^{3} \mathrm{P}$ & 0 & $29299.50+X$ & \multirow{3}{*}{$\begin{array}{l}1103.5 \\
2464.3\end{array}$} & \\
\hline & & 1 & $30402.98+X$ & & 1.494 \\
\hline & & 2 & $32867.3+X$ & & 1.344 \\
\hline $5 f^{2}$ & $5 f^{l}$ & 6 & $30220.0+X$ & & \\
\hline
\end{tabular}


Table 3 (continued)

\begin{tabular}{|c|c|c|c|c|c|}
\hline Configuration & Designation & $J$ & $\begin{array}{l}\text { Level } \\
\left(\mathrm{cm}^{-1}\right)\end{array}$ & $\begin{array}{l}\text { Interval } \\
\left(\mathrm{cm}^{-1}\right)\end{array}$ & Observed $g$ Value \\
\hline \multicolumn{6}{|c|}{ Group A (continued) } \\
\hline \multirow[t]{2}{*}{$5 f 7 p$} & $7 p(5 / 2,1 / 2)$ & 2 & $34996.07+X$ & \multirow[b]{2}{*}{-1433.69} & $0.82_{3}$ \\
\hline & & 3 & $33562.38+X$ & & 0.849 \\
\hline \multirow[t]{2}{*}{$5 / 7 p$} & $7 p(7 / 2,1 / 2)$ & 3 & $38431.66+X$ & \multirow{2}{*}{148.89} & 1.170 \\
\hline & & 4 & $38580.55+X$ & & 1.105 \\
\hline \multirow[t]{4}{*}{$5 / 7 p$} & $7 p(5 / 2,3 / 2)$ & 1 & $44603.10+X$ & \multirow{4}{*}{$\begin{array}{r}-843.7 \\
-1446.3 \\
1388.5\end{array}$} & 0.495 \\
\hline & & 2 & $43759.4+X$ & & 0.988 \\
\hline & & 3 & $42313.10+X$ & & 0.971 \\
\hline & & 4 & $43701.56+X$ & & 1.069 \\
\hline \multirow[t]{4}{*}{$5 / 7 p$} & $7 p(7 / 2,3 / 2)$ & 2 & $49805.5+X$ & \multirow{4}{*}{$\begin{array}{r}-2334.1 \\
-210.5 \\
160.9\end{array}$} & ${ }^{1.03} 0$ \\
\hline & & 3 & $47471.44+x$ & & 1.207 \\
\hline & & 4 & $47260.9+X$ & & 1.14 \\
\hline & & 5 & $47421.8+X$ & & 1.207 \\
\hline $5 f^{2}$ & $5 f^{1} s$ & 0 & $50666.0+X$ & & \\
\hline
\end{tabular}


Table 4. Classified Lines of Th III

\begin{tabular}{|c|c|c|c|c|}
\hline$\lambda(\stackrel{\circ}{)}$ & $\begin{array}{c}\sigma_{\text {vac }} \\
\left(\mathrm{cm}^{-1}\right)\end{array}$ & $\begin{array}{l}\Delta \sigma_{\text {vac }} \\
\left(\mathrm{cm}^{-1}\right)\end{array}$ & Classification & Observed Zeeman Pattern \\
\hline 10702.62 & 9340.95 & +0.5 & $f d^{1} H_{5}^{\circ}-d^{2}{ }^{1} G_{4}$ & \\
\hline 10529.18 & 9494.82 & +0.4 & $f^{2}{ }^{3} \mathrm{H}_{6}-f d^{3} \mathrm{G}_{5}^{0}$ & \\
\hline 10257.59 & 9746.21 & +0.3 & $f^{2}{ }^{3} \mathrm{H}_{5}-f d^{3} \mathrm{G}_{4}^{\circ}$ & \\
\hline 9909.50 & 10088.56 & +0.7 & $f^{2}{ }^{3} \mathrm{H}_{4}-f d^{3} \mathrm{G}_{3}^{\circ}$ & \\
\hline 9685.1 & 10322.3 & +0.6 & $f^{2}{ }^{3} \mathrm{H}_{4}-f d^{3} \mathrm{~F}_{3}^{\circ}$ & \\
\hline 9514.6 & 10507.3 & +0.2 & $f^{2}{ }^{3} \mathrm{~F}_{4}-f d^{3} \mathrm{G}_{5}^{\circ}$ & \\
\hline $9504.11^{b}$ & 10518.88 & +0.20 & $f^{2}{ }^{1} G_{4}-f d{ }^{1} F_{3}^{\circ}$ & \\
\hline $9378.45^{b}$ & 10659.82 & +0.1 & $f^{2}{ }^{3} F_{3}-f d{ }^{3} D_{2}^{\circ}$ & \\
\hline 9135.8 & 10943.0 & +0.2 & $f^{2}{ }^{3} \mathrm{~F}_{2}-f d^{3} \mathrm{D}_{1}^{\circ}$ & \\
\hline 9053.0 & 11043.0 & +0.2 & $f^{2}{ }^{3} \mathrm{~F}_{4}-f d^{3} \mathrm{D}_{3}^{\circ}$ & \\
\hline 8917.64 & 11210.65 & +0.5 & $f^{2}{ }^{{ }^{1} \mathrm{I}_{6}}-f d{ }^{1} \mathrm{H}_{5}^{\circ}$ & \\
\hline 8429.01 & 11860.53 & +0.60 & $f^{2}{ }^{3} \mathrm{~F}_{3}-f d^{3} \mathrm{~F}_{4}^{\circ}$ & \\
\hline 8358.50 & 11960.59 & +0.4 & $f^{2}{ }^{3} \mathrm{H}_{4}-f d^{1} \mathrm{G}_{4}^{\circ}$ & \\
\hline 8342.51 & 11983.51 & +0.4 & $d s^{1} \mathrm{D}_{2}-f s^{3} \mathrm{~F}_{2}^{\circ}$ & \\
\hline 8105.14 & 12334.46 & +0.4 & $f^{2}{ }^{3} \mathrm{H}_{6}-f d^{3} \mathrm{H}_{6}^{\circ}$ & \\
\hline 7920.30 & 12622.32 & +0.8 & $f^{2}{ }^{3} \mathrm{H}_{4}-f s^{3} \mathrm{~F}_{3}^{\circ} ?$ & \\
\hline 7872.26 & 12699.34 & +0.50 & $f^{23} F_{3}-f d^{3} G_{4}^{\circ}$ & \\
\hline 7822.90 & 12779.47 & +0.49 & $f^{2}{ }^{1} \mathrm{D}_{2}-f d^{1} \mathrm{~F}_{3}^{\circ}$ & \\
\hline 7808.04 & 12803.79 & +0.51 & $f^{2}{ }^{3} \mathrm{~F}_{4}-f{ }^{3} \mathrm{~F}_{4}^{\circ}$ & \\
\hline 7493.67 & 13340.93 & +0.93 & $f^{2}{ }^{3} \mathrm{~F}_{3}-f s^{1} \mathrm{~F}_{3}^{\circ} ?$ & \\
\hline 7461.59 & 13398.29 & +0.4 & $f^{2}{ }^{3} \mathrm{H}_{5}-f d^{3} \mathrm{H}_{5}^{\circ}$ & (0) 0.99 \\
\hline 7328.27 & 13642.03 & -0.16 & $f^{2}{ }^{3} F_{4}-f d^{3} G_{4}^{0} ?$ & \\
\hline 7242.47 & 13803.65 & +0.45 & $f^{2}{ }^{3} \mathrm{~F}_{2}-f d^{3} \mathrm{G}_{3}^{\circ}$ & \\
\hline 7121.78 & 14037.57 & +0.56 & $f^{2}{ }^{3} \mathrm{~F}_{2}-f d^{3} \mathrm{~F}_{3}^{\circ}$ & (0) 1.53 \\
\hline 6998.88 & 14284.07 & +0.72 & $f^{2}{ }^{3} \mathrm{~F}_{4}-f s^{1} \mathrm{~F}_{3}^{\circ}$ & (0) 1.54 \\
\hline 6880.15 & 14530.57 & +0.82 & $f^{2}{ }^{3} \mathrm{~F}_{3}-f s^{3} \mathrm{~F}_{4}^{\circ}$ & (0) 1.46 \\
\hline
\end{tabular}

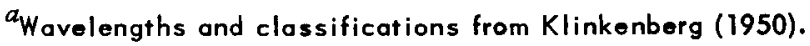

${ }^{b}$ Data from MIT measurements. 
Table 4 (continued)

\begin{tabular}{|c|c|c|c|c|}
\hline$\lambda(\stackrel{\circ}{A})$ & $\begin{array}{c}\sigma_{\mathrm{vac}} \\
\left(\mathrm{cm}^{-1}\right)\end{array}$ & $\begin{array}{l}\Delta \sigma_{\text {vac }} \\
\left(\mathrm{cm}^{-1}\right)\end{array}$ & Classification & Observed Zeeman Pattern \\
\hline 6869.54 & 14553.01 & +0.74 & $f^{2}{ }^{3} \mathrm{~F}_{3}-f d^{1} \mathrm{D}_{2}^{\circ}$ & (0) 1.33 \\
\hline 6802.81 & 14695.76 & +0.29 & $f^{2}{ }^{1} G_{4}-f d^{3} G_{5}^{0} ?$ & (0) 1.44 \\
\hline 6800.96 & 14699.76 & +0.6 & $f^{2}{ }^{3} \mathrm{H}_{5}-f d^{3} \mathrm{G}_{4}^{\circ}$ & (0) 1.18 \\
\hline 6654.00 & 15024.41 & +0.28 & $f^{2}{ }^{\mathrm{I}} \mathrm{D}_{2}-f d^{3} \mathrm{P}_{2}^{\circ}$ & $(0.60) 1.06$ \\
\hline 6599.39 & 15148.74 & +0.2 & $f^{2}{ }^{3} \mathrm{H}_{4}-f d^{3} \mathrm{H}_{4}^{\circ}$ & $(0.23) 0.85$ \\
\hline $6460.72^{c}$ & 15473.88 & +0.78 & $f^{23} \mathrm{~F}_{4}-f^{3} \mathrm{~F}_{4}^{\circ}$ & (0) 1.18 \\
\hline 6374.839 & 15682.34 & -0.08 & $f^{2}{ }^{3} \mathrm{~F}_{2}-f^{3}{ }^{3} \mathrm{~F}_{2}^{\circ}$ & $(0.06) 0.71$ \\
\hline 6335.416 & 15779.93 & +0.07 & $f^{2}{ }^{3} F_{3}-f d^{3} G_{3}^{\circ}$ & $\begin{array}{c}(0.226)^{b}(0.440)^{b} \\
\underline{0.869},{ }^{b} \underline{1.096},{ }^{b} \underline{1.323},^{b} 1.550^{b}\end{array}$ \\
\hline 6242.946 & 16013.66 & -0.01 & $f^{2}{ }^{3} \mathrm{~F}_{3}-f d^{3} \mathrm{~F}_{3}^{\circ}$ & $(0.272) 1.07$ \\
\hline 6140.333 & 16281.26 & -0.2 & $f^{2}{ }^{3} \mathrm{H}_{6}-f d^{3} \mathrm{H}_{5}^{\circ}$ & (0) $1.75, E_{\pi}=0.52$ \\
\hline 6119.465 & 16336.78 & -0.02 & $f^{2}{ }^{3} \mathrm{~F}_{2}-f s^{3} \mathrm{~F}_{3}^{\circ}$ & (0) $(0.39)(0.72) 1.45, \underline{1.78}$ \\
\hline 5895.632 & 16957.02 & 0.00 & $f^{2}{ }^{3} \mathrm{~F}_{4}-f d^{3} \mathrm{~F}_{3}^{\circ}$ & \\
\hline 5883.643 & 16991.57 & 0.00 & $f^{2} \mathrm{I}_{\mathrm{G}_{4}}-f d^{3} \mathrm{~F}_{4}^{\circ}$ & $(0.34)(0.47) 1.23$ \\
\hline 5843.183 & 17109.23 & +0.01 & $f^{2}{ }^{\mathrm{I}_{\mathrm{D}}} \mathrm{D}_{2}-d^{3} \mathrm{P}_{\mathrm{I}}^{\circ}$ & (0) $(0.23) 0.94$ \\
\hline 5814.168 & 17194.61 & -0.09 & $f^{2}{ }^{3} \mathrm{P}_{1}-f d^{3} \mathrm{P}_{2}^{\circ}$ & (0) $1.36, E_{\pi}=0.04$ \\
\hline 5740.955 & 17413.89 & 0.0 & $f^{2}{ }^{3} \mathrm{P}_{2}-f d^{1} \mathrm{~F}_{3}^{\circ}$ & (0) $(0.26) 0.59$ \\
\hline 5715.522 & 17491.37 & +0.02 & $f^{2}{ }^{1} \mathrm{D}_{2}-f d^{3} \mathrm{D}_{3}^{\circ}$ & (0) $(0.10) \underline{1.42,}, E_{\pi}=0.23$ \\
\hline 5606.830 & 17830.45 & -0.04 & $f^{2}{ }^{I} G_{4}-f d^{3} G_{4}^{\circ}$ & (0) 1.07 \\
\hline 5538.106 & 18051.71 & +0.1 & $f^{2}{ }^{1} D_{2}-f d^{3} D_{2}^{\circ}$ & $(0.10) 1.15$ \\
\hline 5500.131 & 18176.35 & +0.04 & $f^{2}{ }^{3} \mathrm{P}_{0}-f d^{3} \mathrm{P}_{1}^{\circ}$ & (0) $1.350, E_{\pi}=0.03$ \\
\hline 5458.969 & 18313.40 & -0.07 & $f^{2}{ }^{3} F_{3}-f s^{3} F_{3}^{\circ}$ & $(0.067) 1.063$ \\
\hline 5447.182 & 18353.03 & -0.11 & $f^{2}{ }^{3} F_{2}-f d^{3} F_{2}^{o}$ & $(0.080) 0.711$ \\
\hline 5412.250 & 18471.48 & -0.17 & $f^{2}{ }^{1} G_{4}-f s^{1} F_{3}^{\circ}$ & (0) 1.16 \\
\hline 5376.132 & 18595.58 & 0.00 & $f^{2}{ }^{3} F_{4}-f d^{1} G_{4}^{\circ}$ & $\underline{(0.80)} 0.58,0.76, \underline{0.98}, \underline{1.20}, 1.38,1.58$ \\
\hline 5214.992 & 19170.16 & -0.01 & $f^{2}{ }^{3} \mathrm{P}_{1}-f d^{3} \mathrm{P}_{0}^{\circ}$ & (0) $1.494, E_{\pi}=0.03$ \\
\hline
\end{tabular}


Table 4 (continued)

\begin{tabular}{|c|c|c|c|c|}
\hline$\lambda(\stackrel{\circ}{A})$ & $\begin{array}{c}\sigma_{v a c} \\
\left(\mathrm{~cm}^{-1}\right)\end{array}$ & $\begin{array}{l}\Delta \sigma_{\text {vac }} \\
\left(\mathrm{cm}^{-1}\right)\end{array}$ & Classification & Observed Zeeman Pattern \\
\hline 5191.519 & 19256.84 & +0.03 & $f^{2}{ }^{3} \mathrm{~F}_{4}-f s^{3} \mathrm{~F}_{3}^{\circ}$ & (0) 1.40 \\
\hline 5185.363 & 19279.70 & -0.08 & $f^{2}{ }^{3} \mathrm{P}_{1}-f d^{3} \mathrm{P}_{1}^{\circ}$ & $(0.142) 1.490$ \\
\hline 5115.76 & 19542.01 & $-0.62^{d}$ & $f p(5 / 2,1 / 2)_{2}-f d^{1} F_{3}^{\circ}$ & \\
\hline 5108.262 & 19570.69 & -0.05 & $f p(7 / 2,1 / 2)_{4}-f d^{1} \mathrm{H}_{5}^{\circ}$ & (0) 0.63 \\
\hline 5085.297 & 19659.07 & +0.1 & $f^{2}{ }^{3} \mathrm{P}_{2}-f{ }^{3} \mathrm{P}_{2}^{\circ}$ & $(0.15) \quad 1.37$ \\
\hline 5084.704 & 19661.36 & -0.03 & $f^{2}{ }^{1} G_{4}-f s^{3} F_{4}^{\circ}$ & $(0.51) 1.21$ \\
\hline 4943.702 & 20222.13 & -0.1 & $f^{2}{ }^{3} \mathrm{P}_{1}-f d^{3} \mathrm{D}_{2}^{\circ}$ & $\underline{(0)}(0.32) \underline{0.89}, 1.21$ \\
\hline 4927.65 & 20288.0 & -0.1 & $d p(3 / 2,1 / 2)_{\uparrow}^{\circ}-d^{2}{ }^{1} \mathrm{~s}_{0}$ & \\
\hline 4917.501 & 20329.87 & 0.06 & $f^{23} \mathrm{~F}_{3}-f d^{3} \mathrm{~F}_{2}^{\circ}$ & \\
\hline 4822.021 & 20732.42 & 0.48 & $f^{2}{ }^{1} \mathrm{D}_{2}-f s^{\top} \mathrm{F}_{3}^{\circ} ?$ & (ㅇ) 0.91 \\
\hline 4780.734 & 20911.46 & -0.06 & $f^{2}{ }^{I} G_{4}-f d^{3} G_{3}^{\circ}$ & (0) 1.58 \\
\hline 4727.850 & 21145.37 & 0.06 & $f^{2}{ }^{1} \mathrm{G}_{4}-f d{ }^{3} \mathrm{~F}_{3}^{\circ}$ & (0) 1.18 \\
\hline 4676.307 & 21378.43 & -0.08 & $f^{2}{ }^{3} \mathrm{P}_{0}-f d^{3} \mathrm{D}_{1}^{\circ}$ & (0) $0.615, E_{\pi}=0.02$ \\
\hline 4597.669 & 21744.08 & 0.0 & $f^{2}{ }^{3} \mathrm{P}_{2}-f d^{3} \mathrm{P}_{1}^{\circ}$ & (0) $1.340, E_{\pi}=0.02$ \\
\hline 4589.280 & 21783.82 & 0.0 & $f^{2}{ }^{3} \mathrm{~F}_{4}-f d^{3} \mathrm{H}_{4}^{\circ}$ & $\begin{array}{l}(0.31)(0.59) 0,0.32,0.59,(0.88)(\underline{1.18)} \\
\underline{0.89}, \underline{1.18}, 1.48,1.77,2.07\end{array}$ \\
\hline 4555.734 & 21944.23 & +0.02 & $f^{2}{ }^{1} \mathrm{D}_{2}-f d^{l} \mathrm{D}_{2}^{\circ}$ & $(0.22)(\underline{0.46)} 0.65, \underline{0.89}, \underline{1.14}, 1.38$ \\
\hline 4518.294 & 22126.06 & $-\sigma .2$ & $f^{2}{ }^{3} \mathrm{D}_{2}-f d^{3} \mathrm{D}_{3}^{\circ}$ & $(0)(0.11) \underline{(0.22)} \underline{1.00}$ \\
\hline 4446.817 & 22481.71 & -0.26 & $f^{2}{ }^{3} \mathrm{P}_{1}-f d^{3} \mathrm{D}_{1}^{\circ}$ & $\underline{(0.84)} 0.63,1.47$ \\
\hline 4406.66 & 22686.6 & +0.1 & $f^{2}{ }^{3} \mathrm{P}_{2}-f d^{3} \mathrm{D}_{2}^{\circ}$ & $(0.30) \quad 1.42$ \\
\hline 4387.840 & 22783.88 & +0.02 & $f^{2}{ }^{1} G_{4}-f d{ }^{1} G_{4}^{\circ}$ & $(0.29)(\underline{0.38)} 1.02$ \\
\hline 4350.818 & 22977.75 & -0.47 & $f p(7 / 2,1 / 2)_{3}-f d^{1} F_{3}^{\circ} ?$ & \\
\hline 4322.724 & 23127.08 & -0.05 & $f p(7 / 2,1 / 2)_{4}-f d^{1} F_{3}^{\circ}$ & (0) 1.20 \\
\hline 4314.319 & 23172.13 & 0.33 & $f^{2} \mathrm{I}_{2}-f d^{3} \mathrm{G}_{3}^{\circ} ?$ & \\
\hline 4048.795 & 24691.76 & 0.00 & $f p(5 / 2,3 / 2)_{4}-/ d^{1} \mathrm{H}_{5}^{\circ}$ & (0) $0.76, E_{\pi}=0.25$ \\
\hline 4028.707 & 24814.87 & -0.4 & $f p(5 / 2,1 / 2)_{2}-f d^{3} \mathrm{D}_{2}^{\circ} ?$ & \\
\hline 3990.730 & 25051.01 & -0.04 & $f^{2}{ }^{1} \mathrm{D}_{2}-f s^{3} \mathrm{~F}_{2}^{\circ}$ & 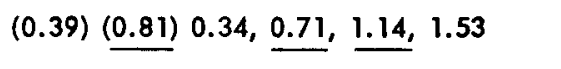 \\
\hline
\end{tabular}




\begin{tabular}{|c|c|c|c|c|}
\hline$\lambda(\AA)$ & $\begin{array}{c}\sigma_{\text {vac }} \\
\left(\mathrm{cm}^{-1}\right)\end{array}$ & $\begin{array}{l}\Delta \sigma_{\text {vac }} \\
\left(\mathrm{cm}^{-1}\right)\end{array}$ & Classification & Observed Zeeman Pattern \\
\hline 3963.460 & 25223.37 & -0.03 & $f p(7 / 2,1 / 2)_{3}-f d^{3} \mathrm{P}_{2}^{\circ}$ & (0) $(0.26)(0.51) \underline{0.61}, 0.87,1.15,1.42$ \\
\hline 3889.153 & 25705.28 & -0.12 & $f^{2}{ }^{1} \mathrm{D}_{2}-f s^{3} \mathrm{~F}_{3}^{\circ}$ & \\
\hline 3849.190 & 25972.15 & +0.03 & $f^{2}{ }^{1} \mathrm{G}_{4}-f d^{3} \mathrm{H}_{4}^{\circ}$ & $g_{1}=1.076, g_{2}=0.987$ \\
\hline 3834.60 & 26071.0 & -0.1 & $s p^{3} \mathrm{P}_{1}^{\circ}-d^{2}{ }^{1} \mathrm{~S}_{0}$ & \\
\hline 3692.392 & 27075.06 & -0.03 & $f p(5 / 2,1 / 2)_{2}-f d^{3} \mathrm{D}_{1}^{\circ}$ & $\underline{(0)}(0.21) 0.62,0.82, \underline{1.03}$ \\
\hline 3665.427 & 27274.21 & 0.01 & $f p(5 / 2,1 / 2)_{3}-f d^{1} \mathrm{D}_{2}^{\circ}$ & (0) 0.77 \\
\hline 3661.440 & 27303.91 & +0.01 & $f p(7 / 2,1 / 2)_{4}-f d^{3} G_{5}^{\circ}$ & (0) $1.50, E_{\pi}=0.32, G_{\sigma}=1.33$ \\
\hline 3610.325 & 27690.47 & -0.15 & $f p(7 / 2,1 / 2)_{3}-f d^{3} D_{3}^{\circ}$ & $(0.21) 1.22$ \\
\hline 3606.255 & 27721.72 & -0.04 & $f^{2}{ }^{1} \mathrm{D}_{2}-f d^{3} \mathrm{~F}_{2}^{\circ}$ & 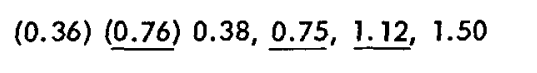 \\
\hline 3595.253 & 27806.55 & -0.1 & $f^{2}{ }^{3} \mathrm{P}_{2}-f d^{3} \mathrm{G}_{3}^{\circ}$ & \\
\hline 3591.003 & 27839.46 & -0.05 & $f p(7 / 2,1 / 2)_{4}-f d^{3} D_{3}^{\circ}$ & (0) 0.97 \\
\hline 3565.251 & 28040.54 & 0.0 & $f^{2}{ }^{3} \mathrm{P}_{2}-f d^{3} \mathrm{~F}_{3}^{\circ}$ & (0) 0.29 \\
\hline 3563.968 & 28050.63 & +0.1 & $d p(5 / 2,1 / 2)_{2}^{\circ}-d s^{1} \mathrm{D}_{2}$ & $(0.18)(\underline{0.40)} 0.80,1.01,1.21,1.40$ \\
\hline 3538.72 & 28250.8 & -0.1 & $f p(7 / 2,1 / 2)_{3}-f d{ }^{3} \mathrm{D}_{2}^{0}$ & (0) 1.13 \\
\hline 3538.69 & 28251.0 & -0.1 & $f p(7 / 2,3 / 2)_{4}-f d^{1} \mathrm{H}_{5}^{\circ}$ & \\
\hline 3531.861 & 28305.62 & -0.4 & $f p(5 / 2,3 / 2)_{2}-f d^{\top} \mathrm{F}_{3}^{\circ}$ & (0) 0.94 \\
\hline 3518.68 & 28411.7 & -0.3 & $f p(7 / 2,3 / 2)_{5}-f d^{1} \mathrm{H}_{5}^{\circ} ?$ & \\
\hline 3516.724 & 28427.46 & -0.1 & $d p(5 / 2,1 / 2)_{3}^{\circ}-d s^{1} D_{2}$ & $\underline{(0)}(0.13)(0.25) \quad \underline{1.34}$ \\
\hline 3507.537 & 28501.91 & +0.12 & $f p(5 / 2,1 / 2)_{3}-f d^{3} G_{3}^{\circ}$ & $(0.063) 0.857$ \\
\hline 3482.381 & 28707.80 & -0.06 & $f p(5 / 2,1 / 2)_{2}-f d^{\top} \mathrm{D}_{2}^{\circ}$ & \\
\hline 3479.049 & 28735.29 & -0.28 & $f p(5 / 2,1 / 2)_{3}-f d{ }^{3} \mathrm{~F}_{3}^{\circ}$ & $(0.49) 0.88$ \\
\hline 3444.192 & 29026.10 & -0.2 & $s p{ }^{3} \mathrm{P}_{1}^{\circ}-d s^{1} \mathrm{D}_{2}$ & (0) 0.97 \\
\hline 3399.796 & 29405.13 & +0.4 & $d p(3 / 2,1 / 2)_{2}^{\circ}-d^{2}{ }^{3} \mathrm{P}_{1} ?$ & \\
\hline 3394.493 & 29451.06 & -0.05 & $f p(7 / 2,1 / 2)_{3}-f d^{3} F_{4}^{\circ}$ & (0) 1.215 \\
\hline 3379.666 & 29580.26 & 0.0 & $f p(7 / 2,3 / 2)_{2}-f d^{I} \mathrm{P}_{1}^{\circ}$ & (0) 1.033 \\
\hline 3377.405 & 29600.06 & +0.03 & $f p(7 / 2,1 / 2)_{4}-f d^{3} F_{4}^{\circ}$ & $(0.285) \quad 1.13$ \\
\hline 3367.672 & 29685.61 & -0.3 & $f^{2}{ }^{3} \mathrm{P}_{2}-f s^{3} \mathrm{~F}_{2}^{\circ} ?$ & \\
\hline 3339.543 & 29935.64 & +0.16 & $f p(5 / 2,1 / 2)_{2}-f d^{3} \mathrm{G}_{3}^{\circ}$ & (0) 0.899 \\
\hline
\end{tabular}


Table 4 (continued)

\begin{tabular}{|c|c|c|c|c|}
\hline$\lambda(\AA)$ & $\begin{array}{c}\sigma_{\mathrm{vac}} \\
\left(\mathrm{cm}^{-1}\right)\end{array}$ & $\begin{array}{l}\Delta \sigma_{\mathrm{vac}} \\
\left(\mathrm{cm}^{-1}\right)\end{array}$ & Classification & Observed Zeeman Pattern \\
\hline 3320.843 & 30104.20 & 0.0 & $d p(3 / 2,1 / 2)_{2}^{\circ}-d s^{3} \mathrm{D}_{2}$ & $(0.36)(0.72) 0.86,1.22$ \\
\hline 3313.680 & 30169.28 & +0.02 & $f p(5 / 2,1 / 2)_{2}-f d^{3} \mathrm{~F}_{3}^{\circ}$ & $\underline{(0)}^{b}(0.175)^{b}(0.341)^{b} \underline{1.366}^{b} 1.013^{b}$ \\
\hline 3300.472 & 30290.00 & -0.02 & $f p(7 / 2,1 / 2)_{3}-f d^{3} G_{4}^{\circ}$ & (0) $E_{\pi}=0.21,1.005$ \\
\hline 3290.587 & 30381.00 & -0.01 & $f p(5 / 2,1 / 2)_{3}-f s^{3} \mathrm{~F}_{2}^{\circ}$ & $(0)(0.125)(0.25) 0.935, \underline{1.06}$ \\
\hline 3284.320 & 30438.96 & +0.05 & $f p(7 / 2,1 / 2)_{4}-f d^{3} \mathrm{G}_{4}^{o}$ & \\
\hline 3284.125 & 30440.77 & 0.0 & $f^{2}{ }^{1} \mathrm{~S}_{0}-f d{ }^{1} \mathrm{P}_{1}^{\circ}$ & (0) 1.009 \\
\hline 3272.279 & 30550.97 & -0.1 & $f p(5 / 2,3 / 2)_{2}-f d^{3} \mathrm{P}_{2}^{\circ}$ & $g_{1}=1.445, g_{2}=0.995$ \\
\hline 3232.061 & 30931.11 & -0.07 & $f p(7 / 2,1 / 2)_{3}-f^{1} \mathrm{~F}_{3}^{\circ}$ & $(0.14)(0.28)(0.427) 1.105$ \\
\hline 3221.206 & 31035.34 & -0.05 & $f p(5 / 2,1 / 2)_{3}-f s^{3} \mathrm{~F}_{3}^{\circ}$ & $\begin{array}{l}(0.22)(0.439)^{b} \underline{(0.655)} 0.4366^{b} \\
0.627, b \underline{0.839},{ }^{b} \underline{1.075},^{b} 1.299^{b}\end{array}$ \\
\hline 3216.571 & 31080.06 & -0.01 & $f p(7 / 2,1 / 2)_{4}-f s^{1} \mathrm{~F}_{3}^{\circ}$ & (0) $1.256, E_{\pi}=0.30$ \\
\hline 3166.446 & 31572.04 & +0.01 & $f p(5 / 2,3 / 2)_{3}-f d^{3} \mathrm{D}_{3}^{\circ}$ & $(0.71) 1.19$ \\
\hline 3159.400 & 31642.45 & +0.2 & $d p(3 / 2,3 / 2)_{2}^{\circ}-d s^{1} \mathrm{D}_{2}$ & (0) 1.08 \\
\hline $3148.077^{b}$ & 31756.23 & -0.2 & $d p(3 / 2,1 / 2)_{2}^{\circ}-d s^{3} \mathrm{D}_{1}$ & $\underline{(0)}^{b}(0.273)^{b} 0.498, b 0.779,^{b} \underline{1.060}^{b}$ \\
\hline 3142.985 & 31807.71 & +0.2 & $f p(7 / 2,3 / 2)_{4}-f d^{1} \mathrm{~F}_{3}^{\circ}$ & (0) 1.22 \\
\hline 3142.304 & 31814.60 & -0.07 & $f p(5 / 2,1 / 2)_{2}-f s^{3} \mathrm{~F}_{2}^{\circ}$ & $(0.176) 0.78$ \\
\hline 3122.341 & 32018.00 & 0.00 & $f p(7 / 2,3 / 2)_{3}-f d^{1} \mathrm{~F}_{3}^{\circ}$ & $(0.36) 1.09$ \\
\hline 3113.897 & 32104.81 & +0.1 & $d p(3 / 2,1 / 2)_{1}^{\circ}-d s^{3} \mathrm{D}_{2}$ & (0) $(0.280) 0.90,1.18, \underline{1.455}$ \\
\hline 3112.338 & 32120.90 & -0.03 & $f p(7 / 2,1 / 2)_{3}-f s^{3} \mathrm{~F}_{4}^{\circ}$ & (0) $1.306, E_{\pi}=0.20$ \\
\hline 3110.152 & 32143.48 & +0.03 & $f p(7 / 2,1 / 2)_{3}-/ d{ }^{l} \mathrm{D}_{2}^{\circ}$ & \\
\hline 3097.977 & 32269.79 & -0.03 & $f p(7 / 2,1 / 2)_{4}-f s^{3} \mathrm{~F}_{4}^{\circ}$ & $(0.43) 1.24$ \\
\hline 3089.642 & 32356.84 & +0.2 & $f^{2}{ }^{3} \mathrm{P}_{2}-f d^{3} \mathrm{~F}_{2}^{\circ}$ & \\
\hline 3083.157 & 32424.90 & -0.01 & $f p(5 / 2,3 / 2)_{4}-f d^{3} \mathrm{G}_{5}^{\circ}$ & (0) $1.64, E_{\pi}=0.50, G_{\sigma}=1.41$ \\
\hline 3078.964 & 32469.05 & 0.00 & $f p(5 / 2,1 / 2)_{2}-/ s^{3} F_{3}^{\circ}$ & (0) $(0.24)(0.49) 1.27,1.53$ \\
\hline 3066.230 & 32603.89 & 0.0 & $d p(3 / 2,1 / 2)_{2}^{\circ}-d^{2}{ }^{1} \mathrm{D}_{2}$ & $(0.23) \underline{(0.46)} 0.55, \underline{0.78}, \underline{1.01}, 1.23$ \\
\hline 3063.194 & 32636.20 & 0.0 & $f p(5 / 2,3 / 2)_{2}-f d^{3} \mathrm{P}_{1}^{\circ}$ & $g_{1}=1.31, g_{2}=0.95$ \\
\hline 3033.053 & 32960.51 & +0.02 & $f p(5 / 2,3 / 2)_{4}-f d^{3} \mathrm{D}_{3}^{\circ}$ & (0) $0.76, E_{\pi}=0.46$ \\
\hline
\end{tabular}


Table 4 (continued)

\begin{tabular}{|c|c|c|c|c|}
\hline$\lambda(\stackrel{\circ}{\AA})$ & $\begin{array}{c}\sigma_{\mathrm{vac}} \\
\left(\mathrm{cm}^{-1}\right)\end{array}$ & $\begin{array}{l}\Delta \sigma_{\text {vac }} \\
\left(\mathrm{cm}^{-1}\right)\end{array}$ & Classification & Observed Zeeman Pattern \\
\hline 3027.753 & 33018.21 & -0.1 & $f p(5 / 2,3 / 2)_{2}-f d^{3} \mathrm{D}_{3}^{\circ}$ & (0) $(0.252)(0.50) 1.25,1.50,1.750$ \\
\hline 3020.039 & 33102.54 & -0.3 & $s p^{3} \mathrm{P}_{1}^{\circ}-s^{2}{ }^{1} \mathrm{~S}_{0}$ & (0) 1.10 \\
\hline 2999.200 & 33332.53 & -0.02 & $f p(5 / 2,3 / 2)_{3}-f d^{3} \mathrm{~F}_{4}^{\circ}$ & (0) 1.77 \\
\hline $2995.837^{b}$ & 33370.07 & -0.21 & $f p(5 / 2,3 / 2)_{1}-f d^{3} \mathrm{P}_{0}^{\circ}$ & (0) 0.53 \\
\hline $2995.75^{b}$ & 33370.9 & -0.14 & $f p(7 / 2,1 / 2)_{3}-f d^{3} \mathrm{G}_{3}^{\circ}$ & $(0.57)(\underline{0.880})$ \\
\hline 2985.998 & 33479.90 & 0.00 & $f p(5 / 2,3 / 2)_{1}-f d^{3} \mathrm{P}_{i}^{\circ}$ & $(0.874) 0.48,1.36$ \\
\hline 2982.398 & 33520.32 & +0.39 & $f p(7 / 2,1 / 2)_{4}-f d^{2} G_{3}^{\circ}$ & (0) 1.35 \\
\hline 2978.662 & 33562.35 & -0.03 & $f p(5 / 2,1 / 2)_{3}-f d^{3} \mathrm{H}_{4}^{\mathrm{O}}$ & (0) $0.941, E_{\pi}=0.14$ \\
\hline 2977.225 & 33578.52 & -0.1 & $f p(5 / 2,3 / 2)_{2}-f d^{3} \mathrm{D}_{2}^{\circ}$ & $(0.21)(0.42) 0.98,1.19$ \\
\hline 2974.896 & 33604.84 & -0.01 & $f p(7 / 2,1 / 2)_{3}-f d^{3} \mathrm{~F}_{3}^{\circ}$ & $\begin{array}{l}(0.451)^{b} 0.687,^{b} 0.832,^{b} 0.982, \\
\underline{1.138},{ }^{b} 1.280,^{b} 1.427^{b}\end{array}$ \\
\hline 2971.08 & 33648.0 & +0.2 & $d p(5 / 2,1 / 2)_{2}^{\circ}-d^{2}{ }^{3} P_{2}$ & $\underline{(0.36)}$ \\
\hline 2961.768 & 33753.78 & +0.04 & $f p(7 / 2,1 / 2)_{4}-f d^{3} F_{3}^{\circ}$ & (0) $1.39, E_{\pi}=0.30$ \\
\hline 2961.485 & 33757.01 & +0.1 & $d p(3 / 2,1 / 2)_{1}^{\circ}-d s^{3} \mathrm{D}_{1}$ & $(0.412)^{b} 0.494^{b} 0.896^{b}$ \\
\hline 2946.94 & 33923.6 & +0.9 & $d p(5 / 2,1 / 2)_{3}^{\circ}-d^{2}{ }^{1} G_{4} ?$ & \\
\hline 2945.32 & 33942.3 & -0.7 & $d p(3 / 2,3 / 2)_{3}^{\circ}-d s^{1} \mathrm{D}_{2} ?$ & (0) 1.23 \\
\hline 2938.178 & 34024.77 & -0.1 & $d p(5 / 2,1 / 2)_{3}^{\circ}-d^{2}{ }^{3} \mathrm{P}_{2}$ & $\underline{(0)}(0.24)(0.48) \underline{0.66,0.90}$ \\
\hline 2932.470 & 34091.00 & +0.01 & $f p(7 / 2,1 / 2)_{4}-f d^{3} \mathrm{H}_{5}^{\circ}$ & (0) $0.86, E_{\pi}=0.25$ \\
\hline 2928.73 & 34134.5 & +0.2 & $d p(5 / 2,1 / 2)_{2}^{\circ}-d s^{3} \mathrm{D}_{3}$ & $(0)(0.16)(0.32) 1.24,1.40,1.56$ \\
\hline $2925.58^{b}$ & 34171.3 & -0.16 & $f p(5 / 2,3 / 2)_{3}-f d^{3} G_{4}^{\circ}$ & $\underline{(0)}^{b}(0.128)^{b}(0.277)^{b} 1.405,{ }^{b}{\underline{1.507^{b}}}^{b}$ \\
\hline 2923.91 & 34190.8 & +0.1 & $d p(3 / 2,1 / 2)_{1}^{\circ}-d^{2}{ }^{3} p_{0}$ & (0) $0.900, E_{\pi}=\mathbf{0 . 0 7}$ \\
\hline $2917.75^{b}$ & 34263.0 & -0.15 & $f p(7 / 2,3 / 2)_{3}-f d^{3} \mathrm{P}_{2}^{\circ}$ & $\underline{(0)}(0.25)(0.50) \underline{0.76}, 1.01,1.26$ \\
\hline $2910.19^{b}$ & 34352.0 & -0.1 & $f p(7 / 2,3 / 2)_{2}-f^{\top}{ }^{\top} \mathrm{F}_{3}^{\circ}$ & (0) $1.121, E_{\pi}=0.09$ \\
\hline 2907.51 & 34383.7 & -0.1 & $s p^{3} \mathrm{P}_{0}^{0}-d^{2}{ }^{3} \mathrm{P}_{1}$ & (0) $1.516, E_{\pi}=0.04$ \\
\hline $2904.254^{b}$ & 34422.16 & -0.1 & $f p(5 / 2,3 / 2) 1-f d^{3} \mathrm{D}_{2}^{\circ}$ & (0) 1.87 \\
\hline $2898.93^{b}$ & 34485.4 & 0.0 & $f p(5 / 2,1 / 2)_{2}-f d^{3} \mathrm{~F}_{2}^{\circ}$ & $(0.110)^{b} 0.792^{b}$ \\
\hline 2896.74 & 34511.5 & +0.1 & $d p(5 / 2,1 / 2)_{3}^{\circ}-d s^{3} D_{3}$ & $\begin{array}{l}(0.21)(0.43)(\underline{0.643})^{b} 0.92,1.130 \\
\underline{1.344}^{b} 1.56\end{array}$ \\
\hline
\end{tabular}


Table 4 (continued)

\begin{tabular}{|c|c|c|c|c|}
\hline$\lambda(\AA)$ & $\begin{array}{c}\sigma_{\text {vac }} \\
\left(\mathrm{cm}^{-1}\right)\end{array}$ & $\begin{array}{l}\Delta \sigma_{\text {vac }} \\
\left(\mathrm{cm}^{-\mathrm{I}}\right)\end{array}$ & Classification & Observed Zeeman Pattern \\
\hline 2888.96 & 34604.4 & 0.0 & $d p(3 / 2,1 / 2)_{1}^{\circ}-d^{2}{ }^{1} \mathrm{D}_{2}$ & (0) $E_{\pi}=0.11,1.087$ \\
\hline 2887.36 & 34623.6 & 0.0 & $s p{ }^{3} \mathrm{P}_{1}^{\circ}-d^{2}{ }^{3} \mathrm{P}_{2}$ & \\
\hline $2871.69^{b}$ & 34812.5 & -0.1 & $f p(5 / 2,3 / 2)_{3}-f s^{1} \mathrm{~F}_{3}^{\circ}$ & $(0.142) 0.999$ \\
\hline 2860.02 & 34954.6 & -0.3 & $d p(3 / 2,3 / 2)_{1}^{\circ}-d s^{1} D_{2} ?$ & \\
\hline 2836.58 & 35243.4 & 0.0 & $f p(7 / 2,1 / 2)_{3}-f d^{1} \mathrm{G}_{4}^{\circ}$ & (0) 1.36 \\
\hline 2824.66 & 35392.1 & -0.2 & $f p(7 / 2,1 / 2)_{4}-f d^{1} \mathrm{G}_{4}^{\circ}$ & $(0.521) 1.07$ \\
\hline 2811.32 & 35560.0 & +0.1 & $f p(5 / 2,3 / 2)_{4}-f d^{3} G_{4}^{\circ}$ & $(0.113) 1.084$ \\
\hline 2789.47 & 35838.6 & +0.2 & $f p(5 / 2,3 / 2)_{2}-f d^{3} D_{1}^{\circ}$ & $\underline{(0)}(0.356) 1.014, \underline{1.370}$ \\
\hline 2784.35 & 35904.5 & -0.1 & $f p(7 / 2,1 / 2)_{3}-f s^{3} \mathrm{~F}_{3}^{\circ}$ & $(0.286) 1.12$ \\
\hline 2778.20 & 35983.9 & -0.3 & $f p(7 / 2,3 / 2)_{4}-f d^{3} \mathrm{G}_{5}^{\circ}$ & $\underline{(0)} 1.35, E_{\pi}=0.27$ \\
\hline 2776.80 & 36002.1 & -0.3 & $f p(5 / 2,3 / 2)_{3}-f s^{3} F_{4}^{o}$ & $\underline{(0)}(0.26)(0.51)(0.76) 1.70, \underline{1.96}$ \\
\hline 2775.06 & 36024.6 & -0.3 & $f p(5 / 2,3 / 2)_{3}-f d^{1} \mathrm{D}_{2}^{\circ}$ & (0) $1.037, E_{\pi}=0.12$ \\
\hline 2772.82 & 36053.7 & +0.2 & $f p(7 / 2,1 / 2)_{4}-f s^{2} \mathrm{~F}_{3}^{\circ}$ & \\
\hline 2765.81 & 36145.1 & 0.0 & $f p(7 / 2,3 / 2)_{5}-f d^{3} \mathrm{G}_{5}^{\circ}$ & (0) $1.198, E_{\pi}=0.09$ \\
\hline $2761.55^{b}$ & 36200.9 & -0.2 & $f p(5 / 2,3 / 2)_{4}-f s^{1} \mathrm{~F}_{3}^{\circ}$ & (0) $1.134, E_{n}=0.07$ \\
\hline 2760.66 & 36212.5 & +0.1 & $d p(5 / 2,1 / 2)_{2}^{\circ}-d^{2}{ }^{3} \mathrm{P}_{1}$ & $\begin{array}{l}\underline{(0)}^{b}(0.302)^{b} \underline{0.892, b} 1.191, b g_{2}= \\
1.192, b g_{1}=1.494^{b}\end{array}$ \\
\hline 2757.12 & 36258.8 & -0.1 & $f p(5 / 2,3 / 2)_{2}-f s^{1} \mathrm{~F}_{3}^{\circ}$ & (0) $1.07, E_{\pi}=0.09$ \\
\hline 2731.61 & 36597.6 & +0.4 & $f p(7 / 2,3 / 2)_{2}-f d^{3} \mathrm{P}_{2}^{\circ}$ & $\underline{(0.74)} \underline{1.045}, 1.415$ \\
\hline 2725.32 & 36682.1 & 0.0 & $f p(5 / 2,3 / 2)_{1}-f d^{3} \mathrm{D}_{1}^{\circ}$ & $(0.130) 0.562$ \\
\hline $2721.82^{b}$ & 36729.3 & -1.1 & $f p(7 / 2,3 / 2)_{3}-f d^{3} \mathrm{D}_{3}^{\circ} ?$ & (0) $1.25, E_{\pi}=0.11$ \\
\hline 2721.35 & 36735.6 & +0.1 & $s p^{3} P_{0}^{\circ}-d s^{3} D_{1}$ & (0) $0.51, E_{\pi}=0.04$ \\
\hline 2708.33 & 36912.2 & +0.3 & $d p(5 / 2,1 / 2)_{2}^{\circ}-d s^{3} \mathrm{D}_{2}$ & (0) $1.19, E_{\pi}=0.10$ \\
\hline 2688.30 & 37187.2 & -1.0 & $s p{ }^{3} P_{1}^{0}-d^{2}{ }^{3} P_{1}$ & $(0.378) 1.14,1.53$ \\
\hline 2686.13 & 37217.2 & 0.0 & $d p(3 / 2,1 / 2)_{2}^{\circ}-d^{2}{ }^{3} \mathrm{~F}_{2}$ & (0) $0.771, E_{\pi}=0.11$ \\
\hline $2680.965^{b}$ & 37288.92 & -0.1 & $d p(5 / 2,1 / 2)_{3}^{\circ}-d s^{3} \mathrm{D}_{2}$ & (0) $1.075, E_{\pi}=0.11$ \\
\hline 2680.90 & 37289.8 & -0.8 & $f p(7 / 2,3 / 2)_{3}-f d^{3} \mathrm{D}_{2}^{\circ} ?$ & \\
\hline
\end{tabular}


Table 4 (continued)

\begin{tabular}{|c|c|c|c|c|}
\hline$\lambda(\stackrel{\circ}{A})$ & $\begin{array}{c}\sigma_{\text {vac }} \\
\left(\mathrm{cm}^{-1}\right)\end{array}$ & $\begin{array}{l}\Delta \sigma_{\text {vac }} \\
\left(\mathrm{cm}^{-1}\right)\end{array}$ & Classification & Observed Zeeman Pattern \\
\hline $2673.677^{b}$ & 37390.56 & -0.27 & $f p(5 / 2,3 / 2)_{4}-f s^{3} \mathrm{~F}_{4}^{\circ}$ & $(0.41)(\underline{0.55)} 1.15$ \\
\hline 2667.92 & 37471.3 & +0.1 & $f p(5 / 2,3 / 2)_{2}-f d^{1} \mathrm{D}_{2}^{\circ}$ & $(0.132) 0.96$ \\
\hline 2666.84 & 37486.4 & +0.1 & $f p(5 / 2,3 / 2)_{3}-f d^{3} \mathrm{~F}_{3}^{\circ}$ & $(0.080) 0.987$ \\
\hline 2638.59 & 37887.8 & +0.1 & $s p{ }^{3} \mathrm{P}_{1}^{0}-d s^{3} \mathrm{D}_{2}$ & (0) $1.211, E_{\pi}=0.07$ \\
\hline 2637.63 & 37901.5 & -0.1 & $d p(5 / 2,3 / 2)_{1}^{\circ}-d s^{1} \mathrm{D}_{2}$ & $\underline{(0)}^{b}(0.236)^{b} \underline{0.764}^{b} 1.001^{b}$ \\
\hline 2636.25 & 37921.4 & +0.4 & $f p(7 / 2,1 / 2)_{3}-f d^{3} \mathrm{~F}_{2}^{\circ}$ & \\
\hline $2635.865^{b}$ & 37926.90 & -0.2 & $d p(5 / 2,1 / 2)_{3}^{0}-d^{2}{ }^{3} F_{4}$ & (0) $1.325, E_{\pi}=0.21$ \\
\hline 2609.16 & 38315.1 & +0.2 & $f p(5 / 2,3 / 2)_{1}-f d^{1} \mathrm{D}_{2}^{\circ}$ & $(0)^{b}(0.403)^{b} 0.512,^{b} 0.936,{ }^{b} 1.315^{b}$ \\
\hline 2601.24 & 38431.7 & 0.0 & $f p(7 / 2,1 / 2)_{3}-f d^{3} \mathrm{H}_{4}^{\mathrm{o}}$ & \\
\hline 2600.59 & 38441.3 & +0.1 & $f p(7 / 2,3 / 2)_{5}-f d^{3} \mathrm{~F}_{4}^{\circ}$ & (0) $1.244, E_{\pi}=0.07$ \\
\hline 2597.26 & 38490.6 & -0.3 & $f p(7 / 2,3 / 2)_{3}-f d^{3} \mathrm{~F}_{4}^{\circ}$ & (0) $1.159, E_{\pi}=0.07$ \\
\hline 2592.29 & 38564.4 & +0.3 & $d p(5 / 2,1 / 2)_{2}^{\circ}-d s^{3} \mathrm{D}_{1}$ & \\
\hline 2591.20 & 38580.6 & 0.0 & $f p(7 / 2,1 / 2)_{4}-f d^{3} \mathrm{H}_{4}^{\circ}$ & $\underline{(0.95)} 1.00$ \\
\hline 2587.25 & 38639.5 & -1.4 & $f p(5 / 2,3 / 2)_{4}-f d^{3} G_{3}^{\circ} ?$ & \\
\hline 2584.40 & 38682.1 & -0.2 & $f p(7 / 2,3 / 2)_{2}-f d^{3} \mathrm{P}_{1}^{\circ}$ & \\
\hline 2583.31 & 38698.5 & -0.3 & $f p(5 / 2,3 / 2)_{2}-f d^{3} G_{3}^{\circ}$ & (0) $\underline{0.64,} E_{\pi}=0.33, G_{\sigma}=0.73$ \\
\hline $2571.612^{b}$ & 38874.47 & -0.28 & $f p(5 / 2,3 / 2)_{4}-f d^{3} F_{3}^{\circ}$ & (0) 1.22 \\
\hline 2567.80 & 38932.2 & -0.4 & $f p(5 / 2,3 / 2)_{2}-f d^{3} \mathrm{~F}_{3}^{\circ}$ & (0) $1.018, E_{\pi}=0.10$ \\
\hline 2564.33 & 38984.9 & 0.0 & $f p(7 / 2,3 / 2)_{5}-f d^{3} \mathrm{H}_{6}^{\circ}$ & (0) 1.067 \\
\hline 2561.25 & 39031.7 & +0.3 & $d p(3 / 2,3 / 2) \stackrel{\rho}{\mathrm{j}}-s^{2}{ }^{1} \mathrm{~S}_{0}$ & (0) 1.24 \\
\hline 2559.11 & 39064.4 & 0.0 & $f p(7 / 2,3 / 2)_{2}-f d^{3} \mathrm{D}_{3}^{\circ}$ & $\underline{(0)}^{b}(0.175)^{b}(0.370)^{b} \underline{1.576},^{b}$ \\
\hline 2555.17 & 39124.6 & -0.2 & $f p(5 / 2,3 / 2)_{3}-f d^{1} G_{4}^{0}$ & (0) $0.983, E_{\pi}=0.05$ \\
\hline 2554.73 & 39131.4 & -0.3 & $f p(5 / 2,3 / 2)_{3}-f s^{3} \mathrm{~F}_{2}^{\circ}$ & $\begin{array}{l}\underline{(0)}^{b}(0.249)^{b}(0.477)^{b} 0.494,^{b} \\
0.732,^{b} 0.979, b 1.218, b \underline{1.448}^{b}\end{array}$ \\
\hline 2549.47 & 39212.1 & +0.1 & $f p(5 / 2,3 / 2)_{4}-f d^{3} \mathrm{H}_{5}^{\circ}$ & (0) $0.95, E_{\pi}=0.10$ \\
\hline 2549.11 & 39217.6 & -0.1 & $d p(3 / 2,1 / 2)_{1}^{\circ}-d^{2}{ }^{3} F_{2}$ & (0) $0.651, E_{\pi}=0.21$ \\
\hline
\end{tabular}

${ }^{b}$ Data from MIT measurements. 
Table 4 (continued)

\begin{tabular}{|c|c|c|c|c|}
\hline$\lambda(\stackrel{\circ}{A})$ & $\begin{array}{c}\sigma_{\text {vac }} \\
\left(\mathrm{cm}^{-1}\right)\end{array}$ & $\begin{array}{l}\Delta \sigma_{\text {vac }} \\
\left(\mathrm{cm}^{-1}\right)\end{array}$ & Classification & Observed Zeeman Pattern \\
\hline 2545.06 & 39280.0 & -0.2 & $f p(7 / 2,3 / 2)_{5}-f d^{3} G_{4}^{O}$ & (0) $1.51, E_{\pi}=0.45$ \\
\hline 2541.86 & 39329.5 & -0.3 & $f p(7 / 2,3 / 2)_{5}-f d^{3} G_{4}^{\circ}$ & (0) $0.89, E_{\pi}=0.34$ \\
\hline 2539.80 & 39361.4 & 0.0 & $d p(5 / 2,3 / 2)_{2}^{\circ}-d s^{1} D_{2}$ & \\
\hline 2536.57 & 39411.5 & -0.1 & $d p(5 / 2,1 / 2)_{2}^{\circ}-d^{2}{ }^{1} \mathrm{D}_{2}$ & $(0.372)^{b} 0.840,{ }^{b} \underline{1.014},^{b} 1.194,^{b} 1.371^{b}$ \\
\hline 2534.89 & 39437.6 & -0.5 & $d p(3 / 2,3 / 2)_{3}^{\circ}-d^{2}{ }^{1} G_{4}$ & \\
\hline 2529.95 & 39514.6 & -0.1 & $d p(5 / 2,3 / 2)_{3}^{\circ}-d s^{1} \mathrm{D}_{2}$ & (0) $1.238, E_{\pi}=0.20$ \\
\hline 2528.33 & 39539.9 & 0.0 & $s p{ }^{3} \mathrm{P}_{1}^{\circ}-d s^{3} \mathrm{D}_{1}$ & $(0.620)^{b} 0.547,^{b} 1.110^{b}$ \\
\hline $2528.31^{b}$ & 39540.2 & 0.0 & $d p(3 / 2,3 / 2)_{3}^{\circ}-d^{2}{ }^{3} \mathrm{P}_{2}$ & \\
\hline 2522.86 & 39625.6 & +0.9 & $f p(7 / 2,3 / 2)_{2}-f d^{3} D_{2}^{\circ} ?$ & \\
\hline 2514.31 & 39760.4 & 0.0 & $f p(7 / 2,3 / 2)_{4}-/ s^{1} \mathrm{~F}_{3}^{\circ}$ & (0) $1.50, E_{\pi}=0.40, G_{\sigma}=1.40$ \\
\hline 2512.69 & 39786.0 & -0.1 & $f p(5 / 2,3 / 2)_{3}-f s^{3} \mathrm{~F}_{3}^{\circ}$ & $(0.256) 1.000$ \\
\hline 2512.54 & 39788.4 & -0.3 & $d p(5 / 2,1 / 2)_{3}^{\circ}-d^{2}{ }^{1} \mathrm{D}_{2}$ & (0) $1.30, E_{\pi}=0.13$ \\
\hline 2511.55 & 39804.1 & -0.1 & $d p(3 / 2,3 / 2)_{2}^{\mathrm{o}}-d^{2}{ }^{3} \mathrm{P}_{1}$ & \\
\hline 2501.08 & 39970.7 & -0.3 & $f p(7 / 2,3 / 2)_{3}-f s^{1} \mathrm{~F}_{3}^{\circ}$ & $\begin{array}{l}(0.18)(0.36)\left(\frac{0.540}{0}\right) \\
1.180^{b}, 1.346^{b}\end{array}$ \\
\hline 2497.56 & 40027.0 & +0.2 & $d p(3 / 2,3 / 2)_{3}^{\circ}-d s^{3} \mathrm{D}_{3}$ & $(0.36) 1.25$ \\
\hline 2497.24 & 40032.1 & -0.1 & $d p(5 / 2,1 / 2)_{2}^{\circ}-d^{2}{ }^{3} \mathrm{~F}_{3}$ & (0) $0.96, E_{\pi}=0.26$ \\
\hline 2475.27 & 40387.4 & 0.0 & $s p{ }^{3} \mathrm{P}_{1}^{\circ}-d^{2}{ }^{1} \mathrm{D}_{2}$ & (0) $0.95, E_{\pi}=0.05$ \\
\hline 2473.93 & 40409.3 & 0.0 & $d p(5 / 2,1 / 2)_{3}^{\circ}-d^{2}{ }^{3} \mathrm{~F}_{3}$ & $(0.106) 1.103$ \\
\hline 2468.19 & 40503.3 & -0.4 & $d p(3 / 2,3 / 2)_{2}^{\circ}-d s^{3} \mathrm{D}_{2}$ & \\
\hline 2467.57 & 40513.5 & +0.2 & $f p(5 / 2,3 / 2)_{4}-f d^{1} \mathrm{G}_{4}^{\circ}$ & $(0.321) 0.97$ \\
\hline 2465.18 & 40552.7 & +0.5 & $d p(3 / 2,3 / 2)_{1}^{\circ}-d^{2}{ }^{3} \mathrm{P}_{2} ?$ & \\
\hline 2463.66 & 40577.8 & -0.2 & $f p(5 / 2,3 / 2)_{2}-f s^{3} \mathrm{~F}_{2}^{\circ}$ & $\begin{array}{l}(0.290)^{b} \\
\underline{0.975},{ }^{b}{\frac{(0.523}{1.223^{b}}}^{b} 0.479, b \underline{0.725}, b\end{array}$ \\
\hline 2441.24 & 40950.3 & +0.1 & $f p(7 / 2,3 / 2)_{4}-f s^{3} \mathrm{~F}_{4}^{\circ}$ & $(0.39) 1.18$ \\
\hline 2431.68 & 41111.3 & +0.2 & $f p(7 / 2,3 / 2)_{5}-f s^{3} \mathrm{~F}_{4}^{\circ}$ & (0) 1.21 \\
\hline $2428.79^{b}$ & 41160.3 & -0.4 & $f p(7 / 2,3 / 2)_{3}-f s^{3} \mathrm{~F}_{4}^{\circ}$ & (0) 1.24 \\
\hline
\end{tabular}


Table 4 (continued)

\begin{tabular}{|c|c|c|c|c|}
\hline$\lambda(\stackrel{\circ}{\AA})$ & $\begin{array}{c}\sigma_{\text {vac }} \\
\left(\mathrm{cm}^{-1}\right)\end{array}$ & $\begin{array}{l}\Delta \sigma_{\text {vac }} \\
\left(\mathrm{cm}^{-\mathrm{I}}\right)\end{array}$ & Classification & Observed Zeeman Pattern \\
\hline 2427.94 & 41174.6 & +0.1 & $f p(5 / 2,3 / 2)_{4}-f s^{3} F_{3}^{\circ}$ & (0) $1.091^{b}$ \\
\hline 2424.54 & 41232.4 & 0.0 & $f p(5 / 2,3 / 2)_{2}-f s^{3} \mathrm{~F}_{3}^{\circ}$ & (0) 1.06 \\
\hline $2413.49^{b}$ & 41421.2 & -0.5 & $f p(5 / 2,3 / 2){ }_{1}-f s^{3} F_{2}^{\circ}$ & (0) 0.94 \\
\hline 2391.48 & 41802.4 & 0.0 & $f p(5 / 2,3 / 2)_{3}-f d^{3} \mathrm{~F}_{2}^{\circ}$ & $\left(\underline{(0)}^{b}(0.209)^{b}(0.419)^{b} 1.002, b 1.196,^{b} \underline{1.384^{b}}\right.$ \\
\hline 2386.81 & 41884.2 & -0.3 & $f p(7 / 2,3 / 2)_{2}-f d^{3} \mathrm{D}_{1}^{\circ} ?$ & \\
\hline 2381.47 & 41978.0 & -0.1 & $d p(5 / 2,3 / 2)_{1}^{\circ}-s^{2} \mathrm{l}_{0}$ & (0) 1.24 \\
\hline 2371.42 & 42155.9 & 0.0 & $d p(3 / 2,3 / 2)_{2}^{\circ}-d s^{3} \mathrm{D}_{1}$ & $\underline{(0)}^{b}(0.515)^{b} 0.507,^{b} 1.019,^{b}{\underline{1.525^{b}}}^{b}$ \\
\hline 2368.91 & 42200.6 & +0.3 & $f p(7 / 2,3 / 2)_{4}-f d^{3} G_{3}^{\circ}$ & (0) 1.44 \\
\hline 2363.06 & 42305.0 & 0.0 & $f p(7 / 2,3 / 2)_{2}-f s^{1} \mathrm{~F}_{3}^{\circ}$ & (0) 1.00 \\
\hline 2362.61 & 42313.1 & 0.0 & $f p(5 / 2,3 / 2)_{3}-f d^{3} \mathrm{H}_{4}^{\circ}$ & \\
\hline 2357.17 & 42410.7 & -0.1 & $f p(7 / 2,3 / 2)_{3}-f d^{3} G_{3}^{\circ}$ & $(0.92) 1.06$ \\
\hline 2355.86 & 42434.4 & +0.3 & $f p(7 / 2,3 / 2)_{4}-f d^{3} \mathrm{~F}_{3}^{\circ}$ & (0) 1.24 \\
\hline 2351.68 & 42509.8 & -0.1 & $d p(5 / 2,3 / 2)_{4}^{\circ}-d^{2}{ }^{1} G_{4}$ & $(0.71) 1.07$ \\
\hline 2344.26 & 42644.3 & -0.3 & $f p(7 / 2,3 / 2)_{3}-f d^{3} \mathrm{~F}_{3}^{\circ} ?$ & (0) 1.11 \\
\hline 2340.58 & 42711.3 & +0.1 & $s p{ }^{3} \mathrm{P}_{2}^{\circ}-d^{2}{ }^{3} \mathrm{P}_{2}$ & (0) 1.36 \\
\hline 2337.29 & 42771.5 & +0.2 & $f p(7 / 2,3 / 2)_{4}-f d^{3} \mathrm{H}_{5}^{\circ}$ & \\
\hline 2335.50 & 42804.3 & -0.1 & $d p(3 / 2,3 / 2)_{3}^{\circ}-d s{ }^{3} \mathrm{D}_{2}$ & (0) 1.22 \\
\hline 2328.54 & 42932.2 & 0.0 & $f p(7 / 2,3 / 2)_{5}-f d^{3} \mathrm{H}_{5}^{\circ}$ & $(0.60)$ \\
\hline 2324.68 & 43003.4 & 0.0 & $d p(3 / 2,3 / 2)_{2}^{\circ}-d^{2}{ }^{\mathrm{I}} \mathrm{D}_{2}$ & (0) 1.03 \\
\hline 2319.52 & 43099.1 & +0.5 & $d p(5 / 2,3 / 2)_{4}^{\circ}-d s^{3} D_{3}$ & (0) 7.16 \\
\hline 2318.57 & 43116.8 & 0.0 & $d p(3 / 2,3 / 2)_{1}^{\circ}-d^{2}{ }^{3} p_{1}$ & \\
\hline 2314.19 & 43198.3 & +0.6 & $s p{ }^{3} \mathrm{P}_{2}^{\circ}-d s^{3} \mathrm{D}_{3}$ & \\
\hline 2311.51 & 43248.5 & -0.2 & $f p(5 / 2,3 / 2)_{2}-f d^{3} \mathrm{~F}_{2}^{\circ}$ & $(0.480)^{b} 0.81$ \\
\hline 2301.18 & 43442.6 & +0.1 & $d p(3 / 2,3 / 2)_{3}^{\circ}-d^{2}{ }^{3} \mathrm{~F}_{4}$ & (0) $1.180^{b}$ \\
\hline 2298.21 & 43498.7 & -0.2 & $d p(5 / 2,3 / 2)_{1}^{\circ}-d^{2}{ }^{3} \mathrm{P}_{2}$ & (0) 1.21 \\
\hline 2297.23 & 43517.3 & 0.0 & $f p(7 / 2,3 / 2)_{2}-f d^{1} D_{2}^{\circ}$ & Does not fit? (0) 0.96 \\
\hline
\end{tabular}


Table 4 (continued)

\begin{tabular}{|c|c|c|c|c|}
\hline$\lambda(\AA)$ & $\begin{array}{c}\sigma_{\text {vac }} \\
\left(\mathrm{cm}^{-1}\right)\end{array}$ & $\begin{array}{c}\Delta \sigma_{\mathrm{vac}} \\
\left(\mathrm{cm}^{-1}\right)\end{array}$ & Classification & Observed Zeeman Pattern \\
\hline 2291.59 & 43624.4 & +0.4 & $d p(3 / 2,3 / 2)_{2}^{\circ}-d^{2}{ }^{3} F_{3}$ & $(0)^{b} 1.177^{b}$ \\
\hline 2287.53 & 43701.8 & +0.2 & $f p(5 / 2,3 / 2)_{4}-f d^{3} \mathrm{H}_{4}^{\circ}$ & $(0.85) 0.95$ \\
\hline 2281.53 & 43816.7 & +0.4 & $d p(3 / 2,3 / 2)_{1}^{\circ}-d s^{3} \mathrm{D}_{2}$ & $(0)^{b} 1.147^{b}$ \\
\hline 2278.78 & 43869.5 & -0.1 & $d p(3 / 2,3 / 2)_{0}^{\circ}-d^{2}{ }^{3} \mathrm{P}_{1}$ & (0) 1.31 \\
\hline 2270.66 & 44026.4 & +1.5 & $d p(5 / 2,1 / 2)_{2}^{\circ}-d^{2}{ }^{3} F_{2} ?$ & \\
\hline 2267.26 & 44092.4 & 0.0 & $f p(5 / 2,3 / 2)_{1}-f d^{3} \mathrm{~F}_{2}^{\circ}$ & \\
\hline 2257.51 & 44282.9 & -0.3 & $f p(7 / 2,3 / 2)_{3}-f d^{1} G_{4}^{\circ}$ & \\
\hline 2257.17 & 44289.5 & -0.5 & $f p(7 / 2,3 / 2)_{3}-f s^{3} \mathrm{~F}_{2}^{\mathrm{O}}$ & \\
\hline 2251.46 & 44401.8 & -0.2 & $d p(5 / 2,1 / 2)_{3}^{\circ}-d^{2}{ }^{3} \mathrm{~F}_{2}$ & \\
\hline 2234.76 & 44733.6 & -0.3 & $f p(7 / 2,3 / 2)_{4}-f s^{3} F_{3}^{\circ}$ & \\
\hline 2234.21 & 44744.6 & -0.3 & $f p(7 / 2,3 / 2)_{2}-f d^{3} \mathrm{G}_{3}^{\circ}$ & \\
\hline 2224.30 & 44943.9 & -0.5 & $f p(7 / 2,3 / 2)_{3}-f s^{3} \mathrm{~F}_{3}^{\circ}$ & \\
\hline 2223.59 & 44958.3 & -0.4 & $d p(5 / 2,3 / 2)_{2}^{\circ}-d^{2}{ }^{3} P_{2}$ & (0) 1.30 \\
\hline 2222.53 & 44979.7 & +1.0 & $f p(7 / 2,3 / 2)_{2}-f d^{3} \mathrm{~F}_{3}^{\circ} ?$ & \\
\hline 2221.51 & 45000.4 & -0.3 & $s p{ }^{3} \mathrm{P}_{1}^{\circ}-d^{2}{ }^{3} \mathrm{~F}_{2}$ & (0) 0.70 \\
\hline 2221.06 & 45009.5 & -0.3 & $d p(5 / 2,3 / 2)_{3}^{\circ}-d^{2}{ }^{1} G_{4}$ & (0) 0.95 \\
\hline 2216.02 & 45111.9 & -0.1 & $d p(5 / 2,3 / 2)_{3}^{\circ}-d^{2}{ }^{3} P_{2}$ & \\
\hline 2208.02 & 45275.3 & -0.5 & $s p{ }^{3} \mathrm{P}_{2}^{\circ}-d^{2}{ }^{3} \mathrm{P}_{1}$ & \\
\hline 2206.62 & 45303.9 & -0.2 & $d p(3 / 2,3 / 2)_{3}^{\circ}-d^{2}{ }^{1} D_{2}$ & (0) 1.25 \\
\hline 2199.74 & 45445.6 & +0.4 & $d p(5 / 2,3 / 2)_{2}^{\circ}-d s^{3} \mathrm{D}_{3}$ & \\
\hline $2198.64^{b}$ & 45468.4 & -0.1 & $d p(3 / 2,3 / 2) \stackrel{\circ}{1}-d s^{3} \mathrm{D}_{1}$ & $(0.78) 0.85$ \\
\hline $2192.38^{b}$ & 45698.2 & -0.3 & $d p(5 / 2,3 / 2)_{3}^{\circ}-d s^{3} \mathrm{D}_{3}$ & \\
\hline 2177.87 & 45902.0 & -0.3 & $d p(3 / 2,3 / 2){ }_{1}^{\circ}-d^{2}{ }^{3} \mathrm{P}_{0}$ & (0) 1.14 \\
\hline 2176.82 & 45924.1 & -0.6 & $d p(3 / 2,3 / 2)_{3}^{\circ}-d^{2}{ }^{3} \mathrm{~F}_{3}$ & \\
\hline 2174.41 & 45975.0 & -0.3 & $s p{ }^{3} \mathrm{P}_{2}^{\circ}-d s{ }^{3} \mathrm{D}_{2}$ & \\
\hline 2170.27 & 46062.8 & -0.7 & $d p(5 / 2,3 / 2)_{1}^{\circ}-d^{2}{ }^{3} \mathrm{P}_{1} ?$ & \\
\hline
\end{tabular}


Table 4 (continued)

\begin{tabular}{|c|c|c|c|c|}
\hline$\lambda(\stackrel{\circ}{A})$ & $\begin{array}{c}\sigma_{\text {vac }} \\
\left(\mathrm{cm}^{-1}\right)\end{array}$ & $\begin{array}{l}\Delta \sigma_{\text {vac }} \\
\left(\mathrm{cm}^{-1}\right)\end{array}$ & Classification & Observed Zeeman Pattern \\
\hline 2162.82 & 46221.4 & +0.1 & $d p(3 / 2,3 / 2)_{0}^{\circ}-d s^{3} \mathrm{D}_{1}$ & \\
\hline 2149.18 & 46514.6 & +0.3 & $d p(5 / 2,3 / 2)_{4}^{\circ}-d^{2}{ }^{3} \mathrm{~F}_{4}$ & \\
\hline 2144.14 & 46624.0 & -0.1 & $f p(7 / 2,3 / 2)_{2}-f s^{3} F_{2}^{o}$ & \\
\hline 2137.76 & 46763.1 & +0.1 & $d p(5 / 2,3 / 2)_{1}^{\circ}-d s^{3} \mathrm{D}_{2}$ & \\
\hline 2115.26 & 47260.6 & -0.3 & $f p(7 / 2,3 / 2)_{4}-f d^{3} \mathrm{H}_{4}^{\circ}$ & \\
\hline 2114.45 & 47278.5 & 0.0 & $f p(7 / 2,3 / 2)_{2}-f s^{3} F_{3}^{\circ}$ & \\
\hline 2108.03 & 47422.5 & +0.7 & $f p(7 / 2,3 / 2)_{5}-f d^{3} \mathrm{H}_{4}^{\circ}$ & \\
\hline 2105.86 & 47471.4 & 0.0 & $f p(7 / 2,3 / 2)_{3}-f d^{3} \mathrm{H}_{4}^{\circ}$ & \\
\hline 2103.59 & 47522.6 & -0.7 & $d p(5 / 2,3 / 2)_{2}^{\circ}-d^{2}{ }^{3} \mathrm{P}_{1}$ & \\
\hline 2099.44 & 47616.6 & -0.1 & $d p(3 / 2,3 / 2)_{2}^{\circ}-d^{2}{ }^{3} F_{2}$ & \\
\hline 2073.04 & 48223.0 & +0.2 & $d p(5 / 2,3 / 2)_{2}^{\circ}-d s^{3} \mathrm{D}_{2}$ & \\
\hline 2064.80 & 48415.2 & 0.0 & $d p(5 / 2,3 / 2)_{1}^{\circ}-d s^{3} \mathrm{D}_{1}$ & \\
\hline 2062.26 & 48475.0 & 0.0 & $s p{ }^{3} \mathrm{P}_{2}^{\circ}-d^{2}{ }^{1} \mathrm{D}_{2}$ & \\
\hline 2046.47 & 48849.0 & 0.0 & $d p(5 / 2,3 / 2)_{1}^{\circ}-d^{2}{ }^{3} \mathrm{P}_{0}$ & \\
\hline 2040.31 & 48996.3 & -0.2 & $d p(5 / 2,3 / 2)_{4}^{\circ}-d^{2}{ }^{3} F_{3}$ & \\
\hline 2039.55 & 49014.5 & +0.3 & $d p(5 / 2,3 / 2)_{3}^{\circ}-d^{2}{ }^{3} \mathrm{~F}_{4}$ & \\
\hline 2036.21 & 49095.1 & -0.5 & $s p^{3} \mathrm{P}_{2}^{0}-d^{2}{ }^{3} \mathrm{~F}_{3}$ & \\
\hline 2029.29 & 49262.5 & -0.2 & $d p(5 / 2,3 / 2)_{1}^{\circ}-d^{2}{ }^{1} \mathrm{D}_{2}$ & \\
\hline 2027.96 & 49294.7 & -0.1 & $f p(7 / 2,3 / 2)_{2}-f d^{3} F_{2}^{\circ}$ & \\
\hline 2004.36 & 49875.1 & +0.1 & $d p(5 / 2,3 / 2)_{2}^{\circ}-d s^{3} \mathrm{D}_{1}$ & \\
\hline 1999.97 & 50007.4 & -0.1 & $s p{ }^{1} \mathrm{P}_{\mathrm{i}}-d^{2}{ }^{1} \mathrm{~S}_{0}$ & \\
\hline 1971.45 & 50724.1 & +1.6 & $d p(5 / 2,3 / 2)_{2}^{\circ}-d^{2}{ }^{1} \mathrm{D}_{2} ?$ & \\
\hline 1965.58 & 50875.6 & -0.2 & $d p(5 / 2,3 / 2)_{3}^{\circ}-d^{2}{ }^{i} \mathrm{D}_{2}$ & \\
\hline 1963.52 & 50929.1 & -0.2 & $d p(3 / 2,3 / 2)_{1}^{\circ}-d^{2}{ }^{3} F_{2}$ & \\
\hline 1947.70 & 51342.7 & -0.4 & $d p(5 / 2,3 / 2)_{2}^{\circ}-d^{2}{ }^{3} F_{3}$ & \\
\hline 1941.88 & 51496.5 & +0.1 & $d p(5 / 2,3 / 2)_{3}^{\circ}-d^{2}{ }^{3} F_{3}$ & \\
\hline 1888.12 & 52962.6 & -0.1 & $s p^{1} \mathrm{P}_{1}^{\circ}-d s^{1} \mathrm{D}_{2}$ & \\
\hline 1802.15 & 55489.3 & +0.2 & $d p(5 / 2,3 / 2)_{3}^{\circ}-d^{2}{ }^{3} F_{2}$ & \\
\hline
\end{tabular}


Table 4 (continued)

\begin{tabular}{|c|c|c|c|c|}
\hline$\lambda(\stackrel{\circ}{\AA})$ & $\begin{array}{c}\sigma_{\mathrm{vac}} \\
\left(\mathrm{cm}^{-1}\right)\end{array}$ & $\begin{array}{l}\Delta \sigma_{\mathrm{vac}} \\
\left(\mathrm{cm}^{-1}\right)\end{array}$ & Classification & Observed Zeeman Pattern \\
\hline 1753.17 & 57039.6 & +0.4 & $s p^{1} P_{1}^{O}-s^{2}{ }^{1} S_{0}$ & \\
\hline 1707.88 & 58552.1 & -7.9 & $s{ }^{1} \mathrm{P}_{1}^{\circ}-d^{2}{ }^{3} \mathrm{P}_{2} ?$ & \\
\hline 1636.06 & 61122.5 & -2.1 & $s p{ }^{1} \mathrm{P}_{1}^{\circ}-d^{2}{ }^{3} \mathrm{P}_{1} ?$ & \\
\hline 1617.48 & 61824.7 & +0.6 & $s p \mathrm{P}_{1}^{\mathrm{P}}-d s^{3} \mathrm{D}_{2}$ & \\
\hline 1564.71 & 63909.8 & -0.3 & $s p^{1} P_{1}^{O}-d^{2}{ }^{3} P_{0}$ & \\
\hline 1554.64 & 64323.5 & -0.3 & $s p^{1} \mathrm{P}_{1}^{\mathrm{O}}-d^{2}{ }^{1} \mathrm{D}_{2}$ & \\
\hline 1450.60 & 68937.2 & +0.1 & $s p^{1} \mathrm{P}_{1}^{\circ}-d^{2}{ }^{3} \mathrm{~F}_{2}$ & \\
\hline
\end{tabular}

\section{THORIUM}

Th II

89 Electrons

Ground State: $1 s^{2} 2 s^{2} 2 p^{6} 3 s^{2} 3 p^{6} 3 d^{10} 4 s^{2} 4 p^{6} 4 d^{10} 4 f^{14} 5 s^{2} 5 p^{6} 5 d^{10} 6 s^{2} 6 p^{6} 6 d 7 s^{2} \quad{ }^{2} \mathrm{D}_{1 / 2}$

This spectrum has been analyzed by McNally, Harrison, and Park and de Bruin, Klinkenberg, and Schuurmans. The levels are taken mostly from McNally's 1945 paper with a few additions from the papers of de Bruin, Klinkenberg, and Schuurmans and several unpublished levels from Klinkenberg and Schuurmans.

\section{REFERENCES}

T. L. de Bruin, Ph. Schuurmans, and P. F. A. Klinkenberg, Z. Physık. 121, 667 (1943). (T)

J. R. McNally, Jr., G. R. Harrison, and H. B. Park, J. Opt. Soc. Am. 32, 334 (1942). (T) (CL) (ZE)

T. L. de Bruin, P. F. A. Klinkenberg, and Ph. Schuurmons, Z. Physzk. 122, 23 (1944). (T) (CL)

J. R. McNally, Jr., J. Opt. Soc. Am. 35, 390 (1945). (T) (CL)

Ph. Schuurmans and P. F. A. Klinkenberg, unpublished material (Oct. 1945). (T) (ZE)

J. N. Lier, Doctoral Dissertation, Amsterdam (1939). (ZE)

P. F. A. Klinkenberg, unpublished material (1950). (T)

G. Racah, unpublished material (1950). (T) (TH)

Y. Eisenberg, Pbysica 18, 177 (1952). (T) (TH)

G. L. Stukenbroeker and J. R. MeNally, Jr., J. Opt. Soc. Am. 43, 36 (1953). (IS)

G. L. Stukenbroeker and J. R. McNally, Jr., unpublished material (1953). (IS) 
Table 5. Energy Levels of Th II

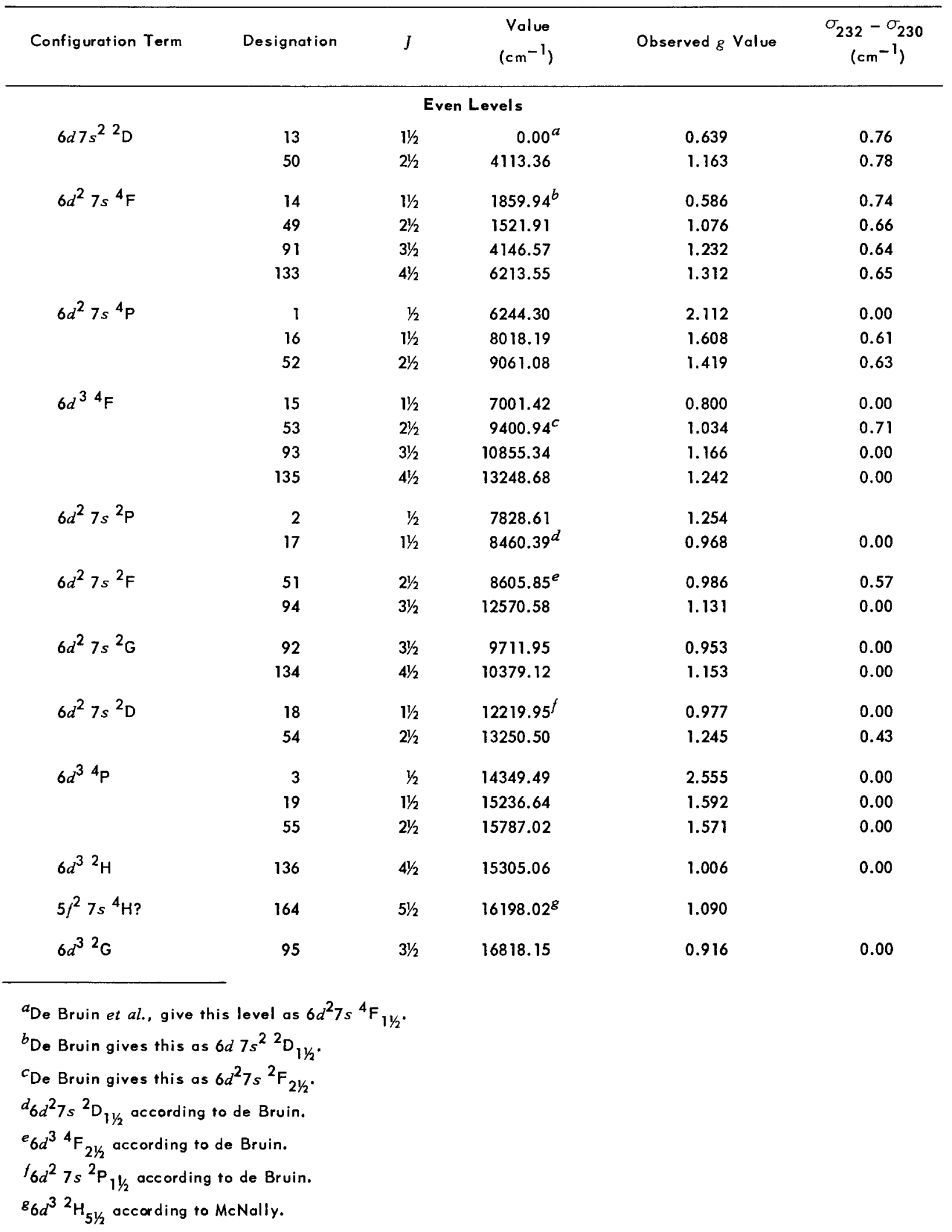


Table 5 (continued)

\begin{tabular}{|c|c|c|c|c|c|}
\hline Configuration Term & Designation & $J$ & $\begin{array}{l}\text { Value } \\
\left(\mathrm{cm}^{-1}\right)\end{array}$ & Observed $g$ Value & $\begin{array}{c}\sigma_{232}-\sigma_{230} \\
\left(\mathrm{~cm}^{-1}\right)\end{array}$ \\
\hline \multicolumn{6}{|c|}{ Even Levels (continued) } \\
\hline \multirow[t]{3}{*}{$5 f^{2} 6 d^{2}{ }^{4} H^{2}$} & 137 & $41 / 2$ & 25246.34 & 0.96 & \\
\hline & 165 & $51 / 2$ & 25629.44 & 1.182 & \\
\hline & 96 & $31 / 2$ & 26072.04 & & \\
\hline \multirow[t]{3}{*}{$5 / 7 s 7 p{ }^{4} \mathrm{G} ?$} & 56 & $2 \frac{1}{2}$ & 26488.64 & 0.776 & \\
\hline & 97 & $31 / 2$ & 27257.19 & 1.032 & 0.55 \\
\hline & 138 & $41 / 2$ & $2752700^{b}$ & 0.93 & \\
\hline \multirow[t]{4}{*}{${ }^{4} \mathrm{~F} ?$} & 20 & $11 / 2$ & 26762.28 & 0.47 & \\
\hline & 57 & $2 \frac{1}{2}$ & 27594.02 & 0.963 & \\
\hline & 99 & $3 \frac{1}{2}$ & $30879.55^{2}$ & 1.213 & \\
\hline & 141 & $4 \frac{1}{2}$ & $31773.09^{1}$ & 1222 & 0.57 \\
\hline \multirow[t]{10}{*}{$5 / 7 s 7 p{ }^{4} \mathrm{~F} ?$} & 21 & $11 / 2$ & 27631.32 & 0.625 & 0.44 \\
\hline & 59 & $2 \frac{1}{2}$ & 28823.68 & 0.987 & 0.53 \\
\hline & 22 & $1 \frac{1}{2}$ & $28011.24^{k}$ & 0.717 & 0.44 \\
\hline & 58 & $2 \frac{1}{2}$ & $28026.38^{l}$ & 1.13 & 0.28 \\
\hline & 60 & $2 \frac{1}{2}$ & 28963.53 & 0.855 & 0.00 \\
\hline & 61 & $21 / 2$ & 29345.93 & 0.935 & \\
\hline & 139 & $4 \frac{1}{2}$ & 29515.19 & 1.030 & 0.30 \\
\hline & 98 & $31 / 2$ & 29873.97 & 1.10 & \\
\hline & 140 & $41 / 2$ & 30452.77 & 1.035 & 0.22 \\
\hline & 62 & $21 / 2$ & 31259.33 & 0.781 & 0.17 \\
\hline \multirow[t]{6}{*}{$5 f 7 s 7 p{ }^{4} G ?$} & 102 & $31 / 2$ & 33209.43 & 1.051 & 0.35 \\
\hline & 145 & $4 \frac{1}{2}$ & 35456.20 & 1.054 & \\
\hline & 171 & $51 / 2$ & 37679.73 & & \\
\hline & 63 & $2 \frac{1}{2}$ & 31754.25 & 0.948 & 0.17 \\
\hline & 100 & $31 / 2$ & 32576.77 & 1.024 & \\
\hline & 166 & $51 / 2$ & 32620.90 & 0.826 & 0.05 \\
\hline \multicolumn{6}{|c|}{$\begin{array}{l}b_{5} f^{2} 6 d{ }^{4} \mathrm{G}_{4 \frac{1}{2}} \text { according to McNally. } \\
{ }^{2} 5 f 7 p 7 s{ }^{4} \mathrm{~F}_{31 / 2} \text { according to de Bruin. } \\
{ }_{5} f_{7 p 7 s}{ }^{4} \mathrm{~F}_{41 / 2} \text { according to de Bruin. } \\
{ }^{k} 5 f 7 p 7 s{ }^{4} \mathrm{~F}_{11 / 2} \text { according to de Bruin. } \\
l_{5} f^{2} 7 s{ }^{2} \mathrm{D}_{21 / 2} \text { according to McNally. }\end{array}$} \\
\hline
\end{tabular}


Table 5 (continued)

\begin{tabular}{|c|c|c|c|c|c|}
\hline Configuration Term & Designation & $J$ & $\begin{array}{l}\text { Value } \\
\left(\mathrm{cm}^{-1}\right)\end{array}$ & Observed $g$ Value & $\begin{array}{c}\sigma_{232}-\sigma_{230} \\
\left(\mathrm{~cm}^{-1}\right)\end{array}$ \\
\hline \multicolumn{6}{|c|}{ Even Levels (continued) } \\
\hline \multirow[t]{2}{*}{$5 / 7 s 7 p^{2} \mathrm{G}^{\circ} ?$} & 101 & $31 / 2$ & 32736.20 & 0.904 & 0.32 \\
\hline & 142 & $4 \frac{1}{2}$ & 33384.46 & 1.14 & \\
\hline \multirow[t]{2}{*}{$5 f 7 s 7 p^{2} \mathrm{D} ?$} & 23 & $1 \frac{1}{2}$ & 32959.61 & 0.874 & 0.12 \\
\hline & 66 & $2 \frac{1}{2}$ & 34543.63 & 1.003 & \\
\hline \multirow[t]{5}{*}{$5 f 6 d 7 p^{4} \mathrm{H}$} & 103 & $31 / 2$ & 33637.26 & 0.830 & 0.19 \\
\hline & 147 & $4 \frac{1}{2}$ & 36125.43 & 1.035 & 0.22 \\
\hline & 173 & $51 / 2$ & $38862.87^{m}$ & 1.083 & 0.19 \\
\hline & 186 & $61 / 2$ & 40724.89 & 1.129 & 0.21 \\
\hline & 64 & $2 \frac{1}{2}$ & 33730.94 & 1.031 & \\
\hline \multirow[t]{5}{*}{$5 f 7 s 7 p^{4} \mathrm{~F} ?$} & 24 & $11 / 2$ & 34019.27 & 0.823 & 0.28 \\
\hline & 69 & $2 \frac{1}{2}$ & 37465.48 & 1.048 & 0.20 \\
\hline & 114 & $31 / 2$ & 39068.75 & 1.114 & 0.25 \\
\hline & 153 & $4 \frac{1}{2}$ & 40367.45 & 1.176 & 0.27 \\
\hline & 65 & $2 \frac{1}{2}$ & 34174.54 & 0.986 & \\
\hline \multirow[t]{8}{*}{$5 f 6 d 7 p^{2} \mathrm{H}$} & 143 & $4 \frac{1}{2}$ & 34270.29 & 0.938 & 0.13 \\
\hline & 168 & $51 / 2$ & 35525.19 & 1.024 & 0.11 \\
\hline & 104 & $3 \frac{1}{2}$ & 34279.34 & 1.047 & \\
\hline & 144 & $4 \frac{1}{2}$ & 34554.37 & 1.069 & \\
\hline & 167 & $51 / 2$ & 34661.76 & 0.998 & 0.19 \\
\hline & 105 & $31 / 2$ & 34726.60 & 0.950 & 0.17 \\
\hline & 4 & $1 / 2$ & $34782.80^{n}$ & 1.67 & \\
\hline & 25 & $11 / 2$ & 35021.39 & 1.042 & \\
\hline \multirow[t]{2}{*}{$5 / 7 s 7 p{ }^{4} \mathrm{D}$} & 5 & $1 / 2$ & 35425.70 & 0.265 & \\
\hline & 28 & $11 / 2$ & 37822.06 & 1.15 & \\
\hline \multirow[t]{4}{*}{$5 f 6 d 7 p^{4} 1$} & 146 & $41 / 2$ & 35545.64 & 0.826 & 0.11 \\
\hline & 170 & $51 / 2$ & 37562.31 & 1.008 & 0.12 \\
\hline & 184 & $61 / 2$ & 38518.10 & 1.111 & 0.17 \\
\hline & 191 & $71 / 2$ & 41099.08 & 1.17 & \\
\hline \multirow[t]{4}{*}{$5 / 6 d 7 p{ }^{4} \mathrm{G} ?$} & 67 & $2 \frac{1}{2}$ & 35741.36 & 0.954 & 0.09 \\
\hline & 107 & $31 / 2$ & 36809.29 & 1.022 & 0.21 \\
\hline & 151 & $41 / 2$ & $39552.29^{\circ}$ & 1.100 & 0.18 \\
\hline & 176 & $51 / 2$ & 40574.81 & 1.140 & 0.22 \\
\hline \multicolumn{6}{|c|}{$\begin{array}{l}m_{5 / 7 s 7 p}{ }^{4} \mathrm{G}_{51 / 2} \text { according to McNally } \\
n_{5} f^{2} 6 d{ }^{4} \mathrm{P}_{1 / 2} \text { according to McNally. } \\
{ }_{5} / 7 s 7 p{ }^{2} \mathrm{G}_{41 / 2} \text { according to McNally. }\end{array}$} \\
\hline
\end{tabular}


Table 5 (continued)

\begin{tabular}{|c|c|c|c|c|c|}
\hline Configuration Term & Designation & $J$ & $\begin{array}{l}\text { Value } \\
\left(\mathrm{cm}^{-1}\right)\end{array}$ & Observed $g$ Value & $\begin{array}{c}\sigma_{232}-\sigma_{230} \\
\left(\mathrm{~cm}^{-1}\right)\end{array}$ \\
\hline \multicolumn{6}{|c|}{ Even Levels (continued) } \\
\hline \multirow[t]{2}{*}{$5 f d 6 d 7 p^{2}$} & 169 & $51 / 2$ & 36310.11 & 0.890 & \\
\hline & 185 & $61 / 2$ & $39085.56^{p}$ & 1.10 & \\
\hline \multirow[t]{3}{*}{$5 f 6 d 7 p{ }^{2} \mathrm{G}$} & 106 & $31 / 2$ & $35878.89^{q}$ & 0.983 & 0.23 \\
\hline & 149 & $4 \frac{1}{2}$ & 37840.91 & 1.07 & 0.11 \\
\hline & 6 & $1 / 2$ & 36040.90 & 1.210 & \\
\hline \multirow[t]{4}{*}{$5 f 6 d 7 p^{2} \mathrm{~F}$} & 68 & $21 / 2$ & 36065.76 & 0.887 & 0.19 \\
\hline & 112 & $31 / 2$ & 38389.43 & 1.088 & 0.34 \\
\hline & 26 & $1 / 2$ & $36328,69^{7}$ & 1.615 & \\
\hline & 7 & $1 / 2$ & 36812.53 & 0.737 & \\
\hline \multirow{2}{*}{$5 f 6 d 7 p^{2} \mathrm{HP}^{2}$} & 148 & $41 / 2$ & $37063.36^{s}$ & 0.999 & 0.22 \\
\hline & 177 & $51 / 2$ & 41223.26 & 1.11 & 0.25 \\
\hline \multirow[t]{2}{*}{$5 / 7 s 7 p^{2} \mathrm{G}^{\mathrm{b}} ?$} & 108 & $31 / 2$ & 37277.39 & 0.881 & 0.15 \\
\hline & 152 & $4 \frac{1}{2}$ & 39895.47 & 1.024 & 0.17 \\
\hline \multirow[t]{4}{*}{$5 f 7 s 7 p{ }^{2} \mathrm{D} ?$} & 27 & $11 / 2$ & 37542.20 & 1.003 & \\
\hline & 74 & $2 \frac{1}{2}$ & 39366.90 & 1.140 & \\
\hline & 183 & $61 / 2$ & 37575.44 & 1.088 & \\
\hline & 109 & $3 \frac{1}{2}$ & 37787.90 & 1.062 & 0.13 \\
\hline \multirow[t]{2}{*}{$5 f 7 s 7 p^{2} \mathrm{~F}$} & 70 & $2 \frac{1}{2}$ & 37945.09 & 0.893 & 0.25 \\
\hline & 116 & $31 / 2$ & 40411.52 & 1.106 & 0.23 \\
\hline${ }^{2} \mathrm{D} ?$ & 71 & $2 \frac{1}{2}$ & 38105.11 & 1.172 & 0.28 or 0.04 \\
\hline \multirow[t]{2}{*}{$5 f 6 d 7 p^{2} \mathrm{G} ?$} & 110 & $31 / 2$ & 38165.40 & 0.981 & 0.27 \\
\hline & 156 & $4 \frac{1}{2}$ & 41909.34 & 1.097 & 0.24 \\
\hline \multirow[t]{2}{*}{$5 f 6 d 7 p^{2} \mathrm{H}$} & 150 & $41 / 2$ & 38179.97 & 0.979 & 0.23 \\
\hline & 175 & $51 / 2$ & 39939.14 & 1.12 & 0.18 \\
\hline \multirow[t]{4}{*}{$5 / 6 d 7 p^{2} G ?$} & 111 & $31 / 2$ & 38291.81 & 0.949 & 0.18 \\
\hline & 157 & $41 / 2$ & 42200.27 & 1.08 & 0.19 \\
\hline & 29 & $11 / 2$ & 38372.19 & 1.200 & 0.15 \\
\hline & 72 & $21 / 2$ & 38728.71 & 1.255 & 0.34 \\
\hline \multicolumn{6}{|c|}{ 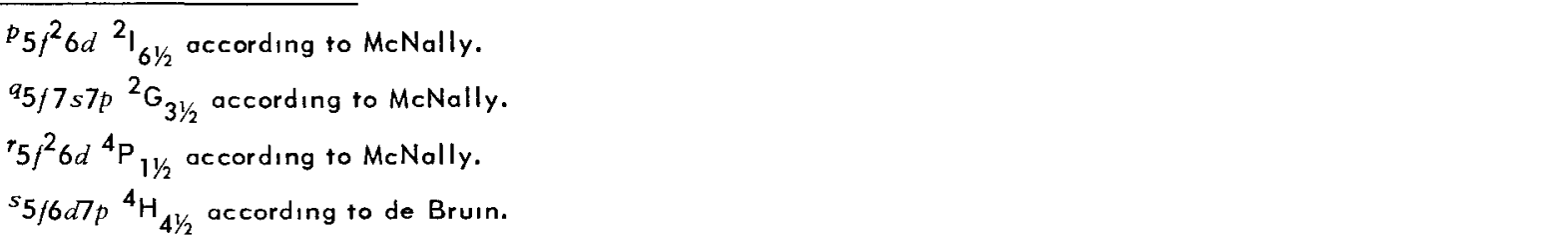 } \\
\hline
\end{tabular}


Table 5 (continued)

\begin{tabular}{|c|c|c|c|c|c|}
\hline Configuration Term & Designation & $J$ & $\begin{array}{l}\text { Value } \\
\left(\mathrm{cm}^{-1}\right)\end{array}$ & Observed $g$ Value & $\begin{array}{c}\sigma_{232}-\sigma_{230} \\
\left(\mathrm{~cm}^{-1}\right)\end{array}$ \\
\hline \multicolumn{6}{|c|}{ Even Levels (continued) } \\
\hline & 172 & $51 / 2$ & 38740.46 & 1.188 & \\
\hline & 30 & $11 / 2$ & 38757.50 & 0.935 & 0.22 \\
\hline \multirow[t]{3}{*}{$5 f 7 s 7 p^{2} \mathrm{D} ?$} & 31 & $1 \frac{1}{2}$ & 38836.24 & 1.013 & 0.23 \\
\hline & 73 & $2 \frac{1}{2}$ & 38863.87 & 0.967 & \\
\hline & 8 & $1 / 2$ & 38867.21 & 0.947 & 0.11 \\
\hline \multirow[t]{3}{*}{$5 f 7 s 7 p{ }^{2} \mathrm{D}$} & 32 & $1 \frac{1}{2}$ & 39150.71 & 0.739 & 0.24 \\
\hline & 82 & $2 \frac{1}{2}$ & 43227.90 & 1.153 & 0.19 \\
\hline & 174 & $51 / 2$ & 39352.30 & 1.05 & 0.13 \\
\hline \multirow[t]{9}{*}{$5 / 6 d 7 p{ }^{4} G ?$} & 115 & $31 / 2$ & 39458.36 & 0.979 & 0.13 \\
\hline & 155 & $4 \frac{1}{2}$ & 41398.58 & 0.983 & 0.12 \\
\hline & 180 & $51 / 2$ & 44177.33 & 1.12 & 0.15 \\
\hline & 75 & $2 \frac{1}{2}$ & 39700.59 & 1.090 & 0.15 \\
\hline & 9 & $1 / 2$ & 39948.52 & 1.542 & \\
\hline & 76 & $2 \frac{1}{2}$ & 40216.41 & 1.024 or 0.412 & $0.26 ?$ \\
\hline & 33 & $1 \frac{1}{2}$ & 40222.94 & 0.738 & \\
\hline & 34 & $11 / 2$ & 40278.15 & 0.705 & 0.00 \\
\hline & 10 & $1 / 2$ & 40523.77 & 0.50 & \\
\hline \multirow[t]{4}{*}{$5 / 6 d 7 p{ }^{4} \mathrm{G} ?$} & 77 & $2 \frac{1}{2}$ & 40644.34 & 0.856 & 0.22 \\
\hline & 117 & $3 \frac{1}{2}$ & 41488.20 & 1.010 & 0.20 \\
\hline & 159 & $41 / 2$ & 43809.23 & 1.102 & 0.25 \\
\hline & 181 & $5 \frac{1}{2}$ & 45735.11 & 1.141 & 0.20 \\
\hline \multirow[t]{3}{*}{$5 / 6 d 7 p^{4} \mathrm{~F} ?$} & 78 & $2 \frac{1}{2}$ & 40923.59 & 0.988 & 0.14 \\
\hline & 121 & $31 / 2$ & 42751.40 & 1.09 & 0.16 \\
\hline & 160 & $4 \frac{1}{2}$ & 45126.29 & 1.21 & 0.12 \\
\hline \multirow[t]{2}{*}{$5 / 0 d 7 p^{2} \mathrm{D} ?$} & 35 & $1 \frac{1}{2}$ & 40991.58 & 1.036 & \\
\hline & 83 & $2 \frac{1 / 2}{2}$ & 44388.70 & 1.158 & 0.05 \\
\hline \multirow[t]{3}{*}{$5 / 6 d 7 p^{2} G ?$} & 154 & $4 \frac{1}{2}$ & 41047.20 & 1.112 & 0.22 \\
\hline & 79 & $2 \frac{1}{2}$ & 41328.38 & 1.101 & 0.31 \\
\hline & 36 & $1 \frac{1}{2}$ & 41676.90 & 1.220 & 0.36 \\
\hline \multirow[t]{2}{*}{$5 / 6 d 7 p^{2} G ?$} & 118 & $31 / 2$ & 41688.48 & 0.940 & 0.14 \\
\hline & 158 & $4 \frac{1}{2}$ & 42955.87 & 1.141 & 0.19 \\
\hline
\end{tabular}


Table 5 (continued)

\begin{tabular}{|c|c|c|c|c|c|}
\hline Configuration Term & Designation & $J$ & $\begin{array}{l}\text { Value } \\
\left(\mathrm{cm}^{-1}\right)\end{array}$ & Observed $g$ Value & $\begin{array}{c}\sigma_{232}-\sigma_{230} \\
\left(\mathrm{~cm}^{-1}\right)\end{array}$ \\
\hline \multicolumn{6}{|c|}{ Even Levels (continued) } \\
\hline & 37 & $11 / 2$ & 41936.60 & 1.095 & \\
\hline & 178 & $51 / 2$ & 42146.57 & 1.158 & 0.18 \\
\hline & 119 & $31 / 2$ & 42222.39 & 0.935 & \\
\hline & 80 & $21 / 2$ & 42352.18 & 1.126 & 0.15 \\
\hline & 120 & $31 / 2$ & 42518.91 & 1.080 & 0.20 \\
\hline & 187 & $61 / 2$ & 42644.94 & 1.10 & 0.22 \\
\hline & 188 & $61 / 2$ & 43014.18 & 0.976 & \\
\hline \multirow{5}{*}{$\begin{array}{c}5 f 6 d 7 p{ }^{4} \mathrm{~F} ? \\
\text { or }{ }^{2} \mathrm{~F} ?\end{array}$} & 81 & $21 / 2$ & 43096.56 & 0.982 & 0.13 \\
\hline & 127 & $31 / 2$ & 45395.10 & 1.216 & \\
\hline & 179 & $51 / 2$ & 43127.24 & 1.037 & 0.23 \\
\hline & 122 & $31 / 2$ & 43246.88 & 1.033 & 0.13 \\
\hline & 123 & $31 / 2$ & 43803.94 & 1.026 & 0.27 \\
\hline \multirow[t]{9}{*}{$5 / 6 d 7 p^{4} \mathrm{D} ?$} & 38 & $11 / 2$ & 43807.65 & 1.211 & \\
\hline & 84 & $21 / 2$ & 44552.68 & 1.182 & \\
\hline & 128 & $31 / 2$ & 46352.29 & & \\
\hline & 11 & $1 / 2$ & 43965.63 & $1.555 ?$ & \\
\hline & 39 & $1 \frac{1}{2}$ & 44300.21 & 1.342 & \\
\hline & 124 & $31 / 2$ & 44503.82 & 1.058 & \\
\hline & 125 & $31 / 2$ & 44807.95 & 1.078 & \\
\hline & 40 & $11 / 2$ & 44889.87 & 1.346 & \\
\hline & 126 & $31 / 2$ & 44898.78 & 1.181 & \\
\hline \multirow[t]{2}{*}{$5 / 6 d 7 p^{2} \mathrm{~F} ?$} & 85 & $21 / 2$ & 45189.70 & 0.674 & \\
\hline & 129 & $3 \frac{1}{2}$ & 46706.20 & 1.081 & \\
\hline \multirow[t]{5}{*}{$5 f 6 d 7 p^{4} \mathrm{~F} ?$} & 86 & $2 \frac{1}{2}$ & 45610.54 & 1.075 & 0.05 \\
\hline & 130 & $31 / 2$ & 47871.40 & 1.194 & \\
\hline & 163 & $41 / 2$ & 50470.20 & 1.18 & \\
\hline & 161 & $4 \frac{1}{2}$ & $45904.41^{t}$ & 1.02 & \\
\hline & 182 & $51 / 2$ & $46216.65^{u}$ & 1.16 & \\
\hline
\end{tabular}


Table 5 (continued)

\begin{tabular}{|c|c|c|c|c|c|}
\hline Configuration Term & Designation & $J$ & $\begin{array}{l}\text { Value } \\
\left(\mathrm{cm}^{-1}\right)\end{array}$ & Observed $g$ Value & $\begin{array}{c}\sigma_{232}-\sigma_{230} \\
\left(\mathrm{~cm}^{-1}\right)\end{array}$ \\
\hline \multicolumn{6}{|c|}{ Even Levels (continued) } \\
\hline \multirow[t]{8}{*}{$5 / 6 d 7 p^{2} \mathrm{D} ?$} & 41 & $1 \frac{1}{2}$ & 46264.18 & 0.891 & 0.08 \\
\hline & 90 & $2 \frac{1}{2}$ & 47324.50 & 1.189 & \\
\hline & 189 & $61 / 2$ & 46378.89 & 1.13 & \\
\hline & 87 & $2 \frac{1}{2}$ & 46581.50 & 1.018 & \\
\hline & 88 & $2 \frac{1}{2}$ & 46603.19 & 1.112 & \\
\hline & 89 & $2 \frac{1 / 2}{2}$ & 46902.54 & 1.143 & \\
\hline & 190 & $61 / 2$ & 46910.85 & 1.18 & 0.17 \\
\hline & 42 & $1 \frac{1}{2}$ & 46935.64 & 0.956 & \\
\hline \multirow[t]{3}{*}{$5 / 6 d 7 p^{2} \mathrm{P} ?$} & 12 & $1 / 2$ & 47145.90 & & \\
\hline & 48 & $1 / 2$ & 51024.73 & 1.270 & \\
\hline & 43 & $11 / 2$ & 47148.76 & & \\
\hline \multirow[t]{7}{*}{$5 f 6 d 7 p^{2} \mathrm{~F} ?$} & 131 & $31 / 2$ & 48298.65 & 1.07 & \\
\hline & 44 & $1 \frac{1}{2}$ & 48689.70 & 0.922 & \\
\hline & 45 & $1 / 2$ & 48817.59 & 0.956 & \\
\hline & 162 & $4 \frac{1}{2}$ & $48854.07^{v}$ & $1.11^{2}$ & \\
\hline & 46 & $11 / 2$ & 49414.54 & 1.003 & \\
\hline & 132 & $31 / 2$ & 49960.54 & 1.248 & \\
\hline & 47 & $11 / 2$ & 50735.65 & & \\
\hline \multicolumn{6}{|c|}{ Odd Levels } \\
\hline \multirow[t]{2}{*}{$5 / 7 s^{2} 2 F^{0}$} & $51^{\circ}$ & $2^{1 / 2}$ & 4490.29 & 0.856 & 0.93 \\
\hline & $94^{\circ}$ & $3 \frac{1}{2}$ & 8378.85 & 1.132 & 0.95 \\
\hline \multirow[t]{4}{*}{$5 / 6 d 7 s^{4} H^{\circ}$} & $93^{\circ}$ & $31 / 2$ & 6168.38 & 0.718 & 0.59 \\
\hline & $130^{\circ}$ & $4 \frac{1}{2}$ & 6700.19 & 1.018 & 0.59 \\
\hline & $151^{\circ}$ & $51 / 2$ & 10189.10 & 1.128 & 0.65 \\
\hline & $162^{\circ}$ & $61 / 2$ & 13406.51 & 1.231 & 0.65 \\
\hline \multirow[t]{4}{*}{$5 / 6 d 7 s^{4} F^{0}$} & $15^{\circ}$ & $1 \frac{1}{2}$ & 6691.44 & 0.492 & 0.54 \\
\hline & $52^{\circ}$ & $2 \frac{1}{2}$ & 7331.51 & 1.061 & 0.56 \\
\hline & $96^{\circ}$ & $31 / 2$ & 9720.30 & 1.173 & 0.59 \\
\hline & $133^{\circ}$ & $41 / 2$ & 12488.34 & 1.245 & 0.65 \\
\hline
\end{tabular}


Table 5 (continued)

\begin{tabular}{|c|c|c|c|c|c|}
\hline Configuration Term & Designation & $J$ & $\begin{array}{l}\text { Value } \\
\left(\mathrm{cm}^{-1}\right)\end{array}$ & Observed $g$ Value & $\begin{array}{c}\sigma_{232}-\sigma_{230} \\
\left(\mathrm{~cm}^{-1}\right)\end{array}$ \\
\hline \multicolumn{6}{|c|}{ Odd Levels (continued) } \\
\hline \multirow[t]{2}{*}{$5 / 6 d 7 s^{2} G^{\circ}$} & $95^{\circ}$ & $3 \frac{1}{2}$ & 9202.29 & וו & 0.62 \\
\hline & $131^{\circ}$ & $4 \frac{1}{2}$ & 9238.04 & 1.086 & 0.60 \\
\hline \multirow[t]{4}{*}{$5 / 6 d 7 s^{4} \mathrm{G}^{\circ}$} & $53^{\circ}$ & $2 \frac{1}{2}$ & 9585.41 & 0.601 & 0.60 \\
\hline & $97^{\circ}$ & $31 / 2$ & 11116.61 & 0.983 & 0.55 \\
\hline & $134^{\circ}$ & $4 \frac{1}{2}$ & 13469.00 & 1.185 & 0.62 \\
\hline & $153^{\circ}$ & $51 / 2$ & 15349.93 & 1.267 & 0.67 \\
\hline \multirow[t]{2}{*}{$5 / 6 d 7 s^{2} H^{\circ}$} & $132^{\circ}$ & $4 \frac{1}{2}$ & 10572.09 & 0.931 & 0.52 \\
\hline & $152^{\circ}$ & $51 / 2$ & 14484.37 & 1.09 & 0.57 \\
\hline \multirow[t]{2}{*}{$5 / 6 d 7 s^{2} \mathrm{~F}^{\circ}$} & $54^{\circ}$ & $2 \frac{1}{2}$ & $10673.16^{w}$ & 1.088 & 0.60 \\
\hline & $100^{\circ}$ & $31 / 2$ & $14791.06^{x}$ & 0.862 & \\
\hline \multirow[t]{2}{*}{$5 / 6 d 7 s^{2} \mathrm{D}^{\circ}$} & $16^{\circ}$ & $1 \frac{1}{2}$ & 11576.43 & 0.832 & 0.55 \\
\hline & $55^{\circ}$ & $2 \frac{1}{2}$ & $12472.18^{y}$ & 0.982 & $0.69 ?$ \\
\hline \multirow[t]{4}{*}{$5 f 6 d 7 s^{4} D^{\circ}$} & $1^{\circ}$ & $1 / 2$ & 11725.55 & 0.255 & \\
\hline & $17^{\circ}$ & $11 / 2$ & 12902.42 & 1.167 & 0.55 \\
\hline & $56^{\circ}$ & $21 / 2$ & 14545.59 & 1.339 & \\
\hline & $101^{\circ}$ & $31 / 2$ & 15453.06 & 1.296 & \\
\hline \multirow[t]{4}{*}{$5 / 6 d^{2}{ }^{4} H^{0}$} & $98^{\circ}$ & $31 / 2$ & 12485.71 & 0.855 & \\
\hline & $136^{\circ}$ & $4 \frac{1}{2}$ & 15242.90 & 1.00 & \\
\hline & $155^{\circ}$ & $51 / 2$ & 17771.10 & 1.10 & \\
\hline & $164^{\circ}$ & $61 / 2$ & 19912.37 & 1.16 & \\
\hline \multirow[t]{2}{*}{$5 f 6 d 7 s^{2} G^{\circ}$} & $99^{\circ}$ & $31 / 2$ & $13818.37^{z}$ & 1.153 & 0.54 \\
\hline & $137^{\circ}$ & $4 \frac{1}{2}$ & 17272.34 & 1.083 & \\
\hline $5 / 6 d^{2} ?{ }^{4} \mathrm{D}^{\circ} ?$ & $2^{\circ}$ & $1 / 2$ & 14101.88 & 0.523 & \\
\hline \multirow[t]{4}{*}{$5 / 6 d^{2} 4 \rho^{\circ}$} & $135^{\circ}$ & $4 \frac{1}{2}$ & 14275.61 & 0.791 & \\
\hline & $154^{\circ}$ & $51 / 2$ & 16564.63 & 0.98 & \\
\hline & $163^{\circ}$ & $61 / 2$ & 18816.93 & 1.10 & \\
\hline & $165^{\circ}$ & $71 / 2$ & 22027.95 & 1.18 & \\
\hline $5 f 6 d 7 s^{2} p^{\circ}$ & $18^{\circ}$ & $1 \frac{1}{2}$ & 15144.78 & 1.366 & \\
\hline $5 / 6 d^{2} ?$ & $57^{\circ}$ & $21 / 2$ & $15173.75^{a a}$ & $1.211 ?$ & \\
\hline $\begin{array}{l}w_{5 / 6 d 7 s}{ }^{2} \mathrm{D}_{21 / 2}^{\circ} \text { accor } \\
x_{5 / 6 d 7 s}{ }^{2} \mathrm{G}_{31 / 2}^{\circ} \text { accor } \\
y_{5 / 6 d 7 s}{ }^{2} \mathrm{~F}_{21 / 2}^{\circ} \text { accor } \\
z_{5 f 6 d 7 s}{ }^{2} \mathrm{~F}_{31 / 2}^{\circ} \text { accor } \\
a a_{5 / 6 d 7 s}{ }^{4} \mathrm{P}_{21 / 2}^{\circ} \text { accor }\end{array}$ & $\begin{array}{l}\text { to McNally. } \\
\text { to McNally. } \\
\text { to McNally. } \\
\text { to McNally an } \\
\text { to MeNally. }\end{array}$ & ruin & & & \\
\hline
\end{tabular}


Table 5 (continued)

\begin{tabular}{|c|c|c|c|c|c|}
\hline Configuration Term & Designation & $J$ & $\begin{array}{l}\text { Value } \\
\left(\mathrm{cm}^{-1}\right)\end{array}$ & Observed $g$ Value & $\begin{array}{c}\sigma_{232}-\sigma_{230} \\
\left(\mathrm{~cm}^{-1}\right)\end{array}$ \\
\hline \multicolumn{6}{|c|}{ Odd Levels (continued) } \\
\hline \multirow[t]{2}{*}{$5 f 6 d 7 s^{4} \mathrm{P}^{0}$} & $3^{\circ}$ & $1 / 2$ & $15324.30^{b b}$ & 2.565 & \\
\hline & $60^{\circ}$ & $21 / 2$ & $17460.70^{c c}$ & 1.51 & 0.55 \\
\hline \multirow[t]{2}{*}{$5 / 6 d 7 s^{2} D^{\circ}$} & $19^{\circ}$ & $11 / 2$ & 15710.92 & $0.643 ?$ & \\
\hline & $58^{\circ}$ & $2 \frac{1}{2}$ & 16033.05 & 1.07 & \\
\hline \multirow[t]{2}{*}{$5 f 6 d^{2}{ }^{4} G^{0}$} & $59^{\circ}$ & $21 / 2$ & $16136.09 ?$ & & \\
\hline & $102^{\circ}$ & $31 / 2$ & 16906.72 & 0.950 & \\
\hline $5 f 6 d^{2}{ }^{4} D^{\circ} ?$ & $20^{\circ}$ & $11 / 2$ & $17121.64^{d d}$ & 1.277 & \\
\hline $5 f 6 d^{2} ?$ & $61^{\circ}$ & $21 / 2$ & 17983.41 & 0.995 & \\
\hline \multirow[t]{4}{*}{$5 f 6 d^{2}{ }^{4} F^{0}$} & $21^{\circ}$ & $1 \frac{1}{2}$ & 18214.47 & 0.876 & \\
\hline & $62^{\circ}$ & $2 \frac{1}{2}$ & 19248.32 & 0.931 & \\
\hline & $108^{\circ}$ & $31 / 2$ & 21682.70 & 1.248 & \\
\hline & $142^{\circ}$ & $4 \frac{1}{2}$ & 23749.76 & 1.34 & \\
\hline $5 / 6 d 7 s^{2} \mathrm{~F}^{\mathrm{O}} ?$ & $103^{\circ}$ & $31 / 2$ & 18973.80 & 1.12 & \\
\hline \multirow[t]{2}{*}{$5 / 6 d^{2}{ }^{2} \mathrm{D}^{\circ} ?$} & $22^{\circ}$ & $11 / 2$ & 19050.87 & 0.888 & \\
\hline & $104^{\circ}$ & $31 / 2$ & 19929.15 & & \\
\hline $5 f 6 d^{2}{ }^{2} \mathrm{G}^{\circ} ?$ & $105^{\circ}$ & $31 / 2$ & $20080.68^{e e}$ & 1.142 & \\
\hline \multirow[t]{5}{*}{$5 f 6 d^{2}{ }^{4} G^{o b}$} & $63^{\circ}$ & $21 / 2$ & 20120.14 & 0.743 & \\
\hline & $109^{\circ}$ & $31 / 2$ & 22685.50 & 0.981 & 0.00 \\
\hline & $144^{\circ}$ & $4 \frac{1}{2}$ & 24757.59 & 1.13 & 0.00 \\
\hline & $158^{\circ}$ & $51 / 2$ & 26770.52 & 1.250 & \\
\hline & $64^{\circ}$ & $2 \frac{1}{2}$ & 20686.23 & 0.945 & \\
\hline $5 f 6 d^{2}{ }^{2} G^{\circ} ?$ & $106^{\circ}$ & $31 / 2$ & 20852.71 & & \\
\hline \multirow[t]{2}{*}{$5 / 6 d 7 s^{2} \mathrm{H}^{\circ}$} & $138^{\circ}$ & $4 \frac{1}{2}$ & 20989.85 & 0.953 & \\
\hline & $156^{\circ}$ & $51 / 2$ & 22014.94 & 1.10 & \\
\hline \multirow[t]{2}{*}{$5 / 6 d^{2}{ }^{2} \mathrm{G}^{\circ} ?$} & $107^{\circ}$ & $31 / 2$ & $21207.61^{f f}$ & & \\
\hline & $65^{\circ}$ & $2 \frac{1}{2}$ & 21297.45 & 0.767 & 0.00 \\
\hline $5 / 6 d^{2}{ }^{2} G^{\circ} ?$ & $139^{\circ}$ & $4 \frac{1}{2}$ & 22139.64 & 1.090 & \\
\hline $\begin{array}{l}{ }^{b b_{5}} d_{6} d^{2}{ }^{4} \mathrm{P}_{1 / 2}^{\circ} \text { accordin } \\
c c_{5 f 6 d^{2}}{ }^{4} \mathrm{P}_{21 / 2}^{\circ} \text { accord } \\
d d_{5 f 6 d 7 s^{2} \mathrm{P}_{11 / 2}^{\circ} \text { accor }} \\
e e_{5 f 6 d^{2}}{ }^{4} \mathrm{~F}_{31 / 2}^{\circ} \text { accord } \\
t f_{5 f 6 d^{2}}{ }^{4} \mathrm{D}_{31 / 2}^{\circ} \text { accord }\end{array}$ & $\begin{array}{l}\text { MeNally. } \\
\text { MeNally. } \\
\text { to MeNally. } \\
\text { MeNally. } \\
\text { MeNally }\end{array}$ & & & & \\
\hline
\end{tabular}


Table 5 (continued)

\begin{tabular}{|c|c|c|c|c|c|}
\hline Configuration Term & Designation & $J$ & $\begin{array}{l}\text { Value } \\
\left(\mathrm{cm}^{-1}\right)\end{array}$ & Observed $g$ Value & $\begin{array}{c}\sigma_{232}-\sigma_{230} \\
\left(\mathrm{~cm}^{-1}\right)\end{array}$ \\
\hline \multicolumn{6}{|c|}{ Odd Levels (continued) } \\
\hline & $66^{\circ}$ & $2 \frac{1}{2}$ & 22513.32 & & \\
\hline \multirow[t]{3}{*}{$5 f 6 d^{2}{ }^{2} \mathrm{H}^{\circ} \mathrm{P}$} & $140^{\circ}$ & $4 \frac{1}{2}$ & 22642.20 & 1.043 & \\
\hline & $159^{\circ}$ & $5 \frac{1}{2}$ & 27378.53 & 1.15 & \\
\hline & $23^{\circ}$ & $11 / 2$ & 23012.03 & 1.017 & 0.10 \\
\hline \multirow[t]{3}{*}{$5 f 6 d^{2}{ }^{4} D^{0}$} & $24^{\circ}$ & $11 / 2$ & 23372.57 & 1.067 & 0.21 \\
\hline & $67^{\circ}$ & $2 \frac{1}{2}$ & 24463.86 & 1.396 & 0.07 \\
\hline & $111^{\circ}$ & $31 / 2$ & 27249.56 & 1.382 & 0.05 \\
\hline \multirow[t]{4}{*}{$5 f 6 d^{2}{ }^{2} \mathrm{G}^{\mathrm{O}_{3}}$} & $157^{\circ}$ & $51 / 2$ & 23624.03 & & \\
\hline & $141^{\circ}$ & $4 \frac{1}{2}$ & $23730.81^{g g}$ & 1.186 & \\
\hline & $25^{\circ}$ & $1 \frac{1}{2}$ & 24132.05 & 1.429 & 0.10 \\
\hline & $26^{\circ}$ & $1 \frac{1}{2}$ & 2441460 & 1.105 & 0.29 \\
\hline $5 f 6 d^{2}{ }^{4} F^{O_{3}}$ & $143^{\circ}$ & $4 \frac{1}{2}$ & 2460468 & & \\
\hline \multirow[t]{3}{*}{$6 d 7 s 7 p^{2}$} & $68^{\circ}$ & $21 / 2$ & 24873.99 & 1.081 & 0.33 \\
\hline & $110^{\circ}$ & $31 / 2$ & 24982.42 & 0.990 & 0.00 \\
\hline & $27^{\circ}$ & $11 / 2$ & 25188.08 & 0.937 & 0.12 \\
\hline \multirow[t]{3}{*}{${ }^{2} \mathrm{PO}^{\mathrm{O}_{3}}$} & $4^{\circ}$ & $1 / 2$ & $25266.58^{b b}$ & 0.725 & \\
\hline & $69^{\circ}$ & $2 \frac{1}{2}$ & 25440.26 & 1.138 & 0.05 \\
\hline & $70^{\circ}$ & $2 \frac{1}{2}$ & $26424.53^{22}$ & 1.169 & 0.03 \\
\hline \multirow[t]{6}{*}{$5 f 6 d^{2}{ }^{4} \mathrm{D}^{\circ} ?$} & $5^{\circ}$ & $1 / 2$ & 26626.45 & 0.645 & 0.18 \\
\hline & $32^{\circ}$ & $11 / 2$ & 28720.86 & 1.162 & \\
\hline & $73^{\circ}$ & $2 \frac{1}{2}$ & 30972.18 & 1.217 & \\
\hline & $113^{\circ}$ & $31 / 2$ & 31800.21 & 1.168 & 0.14 \\
\hline & $30^{\circ}$ & $1 \frac{1}{2}$ & 26965.21 & 1.384 & 0.08 \\
\hline & $31^{\circ}$ & $11 / 2$ & 27403.17 & 1.002 & \\
\hline \multirow[t]{2}{*}{$5 / 6 d^{2}{ }^{2} \mathrm{~F}^{\mathrm{O}_{3}}$} & $71^{\circ}$ & $2 \frac{1}{2}$ & $28243.84^{11}$ & 0.922 & 0.31 \\
\hline & $112^{\circ}$ & $31 / 2$ & 30994.30 & 1.199 & \\
\hline
\end{tabular}

${ }^{88} 5 / 6 d^{2}{ }^{4} G_{41 / 2}^{\circ}$ according to McNally.

${ }^{b b} 7 p 7 s^{2} 2 \mathrm{P}_{1 / 2}^{\circ}$ occording to MeNally.

${ }^{21} 5 / 6 d^{2}{ }^{4} \mathrm{D}_{2 \frac{1}{2}}^{\circ}$ according to McNally.

${ }^{11} 6 d^{2} 7 p{ }^{4} \mathrm{G}_{21 / 2}^{\mathrm{O}}$ according to de Bruin. 
Table 5 (continued)

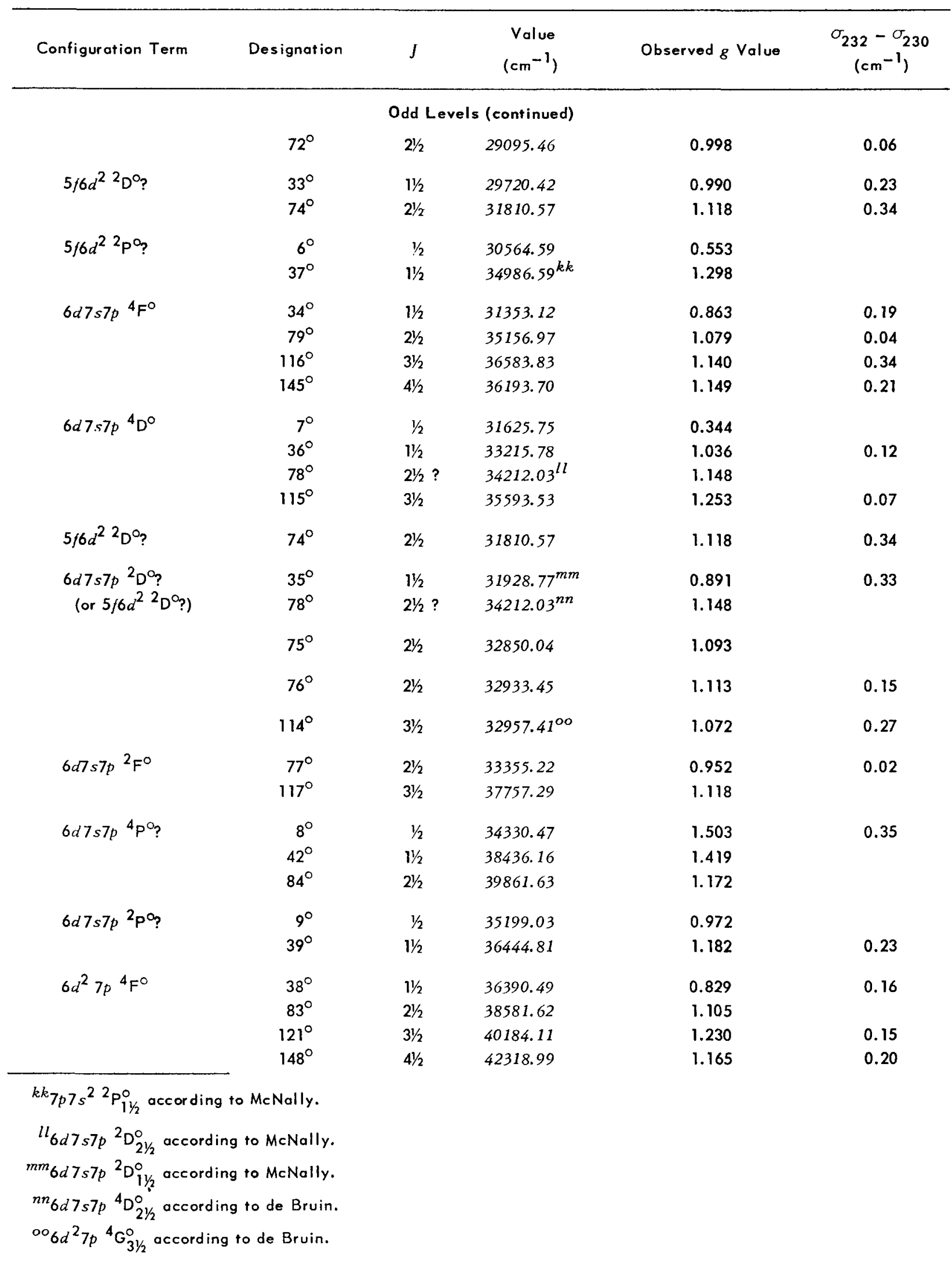


Table 5 (continued)

\begin{tabular}{|c|c|c|c|c|c|}
\hline Configuration Term & Designation & $J$ & $\begin{array}{l}\text { Value } \\
\left(\mathrm{cm}^{-1}\right)\end{array}$ & Observed $g$ Value & $\begin{array}{c}\sigma_{232}-\sigma_{230} \\
\left(\mathrm{~cm}^{-1}\right)\end{array}$ \\
\hline \multicolumn{6}{|c|}{ Odd Levels (continued) } \\
\hline \multirow[t]{2}{*}{$6 d 7 s 7 p^{2} \mathrm{D}^{\mathrm{O}_{p}}$} & $40^{\circ}$ & $1 \frac{1}{2}$ & 36581.57 & 1.107 & 0.15 \\
\hline & $81^{\circ}$ & $2 \frac{1}{2}$ & $37846.15^{p p}$ & 1.165 & \\
\hline \multirow[t]{4}{*}{$6 d^{2} 7 p{ }^{4} \mathrm{G}^{\circ}$} & $80^{\circ}$ & $2 \frac{1}{2}$ & $36687.99^{q q}$ & 1.049 & 0.21 \\
\hline & $118^{\circ}$ & $3 \frac{1}{2}$ & 38764.61 & 1.098 & 0.22 \\
\hline & $147^{\circ}$ & $41 / 2$ & 39443.02 & 1.145 & 0.22 \\
\hline & $161^{\circ}$ & $51 / 2$ & 41448.00 & 1.267 & 0.32 \\
\hline \multirow[t]{4}{*}{$6 d^{2} 7 p^{4} D^{\infty}$} & $10^{\circ}$ & $1 / 2$ & 37130.35 & 0.521 & 0.28 \\
\hline & $43^{\circ}$ & $1 \frac{1}{2}$ & 40472.42 & 1.249 & 0.20 \\
\hline & $86^{\circ}$ & $2 \frac{1}{2}$ & 42219.77 & 1.168 & 0.00 \\
\hline & $126^{\circ}$ & $31 / 2$ & 44650.52 & 1.234 & \\
\hline \multirow[t]{2}{*}{${ }^{4} \mathrm{~F}^{\mathrm{O}}$} & $146^{\circ}$ & $4 \frac{1}{2}$ & 37569.79 & 1.29 & \\
\hline & $41^{\circ}$ & $11 / 2$ & $37601.71^{r r}$ & 1.267 & 0.06 \\
\hline${ }^{2} \mathrm{~S}^{\circ}$ & $11^{\circ}$ & $1 / 2$ & $37608.01^{s s}$ & 2.238 & \\
\hline \multirow[t]{2}{*}{${ }^{2} \mathrm{P}^{\circ}$} & $12^{\circ}$ & $1 / 2$ & $37716.37^{t t}$ & 0.690 & 0.19 \\
\hline & $82^{\circ}$ & $2 \frac{1}{2}$ & $38493.72^{u u}$ & 1.217 & \\
\hline \multirow[t]{2}{*}{${ }^{2} \mathrm{~F}^{\circ}$} & $119^{\circ}$ & $3 \frac{1}{2}$ & 39396.54 & 1.131 & 0.00 \\
\hline & $120^{\circ}$ & $31 / 2$ & 39585.43 & $1.219 ?$ & \\
\hline${ }^{4} G^{\circ} ?$ & $160^{\circ}$ & $51 / 2$ & 39606.17 & 1.259 & 0.23 \\
\hline \multirow[t]{2}{*}{$6 d 7 s 7 p^{2} \mathrm{~F}^{\circ}$} & $85^{\circ}$ & $21 / 2$ & 40654.54 & 0.943 & \\
\hline & $122^{\circ}$ & $31 / 2$ & $40707.40^{v v}$ & 1.073 & 0.13 \\
\hline \multirow[t]{4}{*}{$6 d^{2} 7 p{ }^{4} D^{o b}$} & $13^{\circ}$ & $1 / 2$ & 40825.58 & 0.261 & \\
\hline & $45^{\circ}$ & $11 / 2$ & 42418.19 & 1.304 & \\
\hline & $87^{\circ}$ & $2 \frac{1}{2}$ & 43382.76 & 1.203 & 010 \\
\hline & $127^{\circ}$ & $31 / 2$ & 45689.25 & 1.261 & \\
\hline $6 d^{2} 7 p^{4} \mathrm{~s}^{\circ}$ & $44^{\circ}$ & $11 / 2$ & 42112.93 & 1.467 & \\
\hline 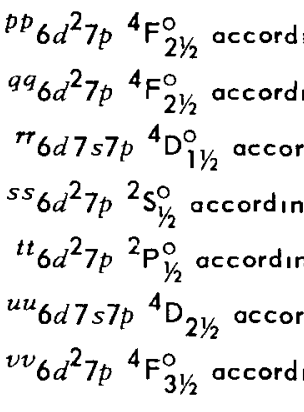 & $\begin{array}{l}\text { McNally. } \\
\text { de Bruin. } \\
\text { to MeNally. } \\
\text { MeNally. } \\
\text { McNally. } \\
\text { to McNally. } \\
\text { McNally. }\end{array}$ & & & & \\
\hline
\end{tabular}


Table 5 (continued)

\begin{tabular}{|c|c|c|c|c|c|}
\hline Configuration Term & Designation & $J$ & $\begin{array}{l}\text { Value } \\
\left(\mathrm{cm}^{-1}\right)\end{array}$ & Observed $g$ Value & $\begin{array}{c}\sigma_{232}-\sigma_{230} \\
\left(\mathrm{~cm}^{-1}\right)\end{array}$ \\
\hline \multicolumn{6}{|c|}{ Odd Levels (continued) } \\
\hline & $123^{\circ}$ & $31 / 2$ & $42944.46^{w w}$ & 1.246 & 0.13 \\
\hline \multirow[t]{5}{*}{$6 d^{2} 7 p^{4} \dot{\mathrm{P}}^{\circ}$} & $14^{\circ}$ & $1 / 2$ & 43744.06 & 2.219 & \\
\hline & $46^{\circ}$ & $1 \frac{1}{2}$ & 43287.63 & 1.564 & \\
\hline & $89^{\circ}$ & $2 \frac{1}{2}$ & 45221.48 & 1.347 & \\
\hline & $124^{\circ}$ & $3 \frac{1}{2}$ & 43773.66 & $1.046^{?}$ & \\
\hline & $125^{\circ}$ & $31 / 2$ & $43774.06^{?}$ & & \\
\hline $6 d^{2} 7 p{ }^{2} \mathrm{~F}^{\mathrm{O}}$ & $88^{\circ}$ & $2 \frac{1}{2}$ & 44821.86 & 0.999 & \\
\hline \multirow[t]{3}{*}{${ }^{2} G^{\circ} ?$} & $149^{\circ}$ & $4 \frac{1}{2}$ & 45489.12 & 1.089 & \\
\hline & $47^{\circ}$ & $1 \frac{1}{2}$ & 45697.84 & 1.18 & \\
\hline & $90^{\circ}$ & $21 / 2$ & 46155.67 & 1.152 & \\
\hline${ }^{2} G^{\circ}$ & $150^{\circ}$ & $4 \frac{1}{2}$ & 46368.03 & 1.088 & \\
\hline \multirow[t]{3}{*}{$2 \mathrm{pop}^{2}$} & $48^{\circ}$ & $1 / 2$ & 46423.77 & 1.118 & \\
\hline & $91^{\circ}$ & $2 \frac{1}{2}$ & 49289.44 & 1.208 & \\
\hline & $49^{\circ}$ & $11 / 2$ & 51528.60 & 0.925 & \\
\hline \multirow[t]{3}{*}{$5 f^{2} 7 p^{2} \mathrm{~F}^{\mathrm{O}}$} & $92^{\circ}$ & $21 / 2$ & 51946.85 & 0.937 & \\
\hline & $129^{\circ}$ & $31 / 2$ & 54497.50 & 1.126 & \\
\hline & $50^{\circ}$ & $1 / 2$ & 53949.20 & & \\
\hline
\end{tabular}

${ }^{w w} 6 d 7 s 7 p{ }^{4} \mathrm{P}_{\mathrm{i} 1 / 2}^{0}$ according to McNally.

The data in Table 6 are taken from the following sources:

C M. Fred, MS Thesis, M. Ference, Jr., MS Thesis, and R. B. Bowersox, MS Thesis, University of Chicago (1934).

E,V J. M. Eder and E. Valenta, Atlas typischer Spektren, Vienna, A. Holder, 1911.

$K$ P. F. A. Klinkenberg, unpublished wavelength list and unpublished Zeeman data.

L J. N. Lier, Doctoral Dissertation, Amsteriam (1939).

Me J. R. MeNally, Jr., J. Opt. Soc, Am. 35, 390 (1945).

M MIT data are taken from either MIT wavelength tables or MIT card files on thorium. MIT Zeeman data are also taken from J. R. McNally, Jr., G. R. Harrison, and H. B. Park, J Opt. Soc. Am. 32, 334 (1942).

S Ph. Schuurmans, Doctoral Dissertation, Amsterdam (1946).

$\checkmark$ Vatican data from A. Gatterer and J. Junkes, Spektren der Seltenen Erden, p 329-347, Specola Vaticana, Vatican City, 1945. 
Table 6. Classified Lines of Th II

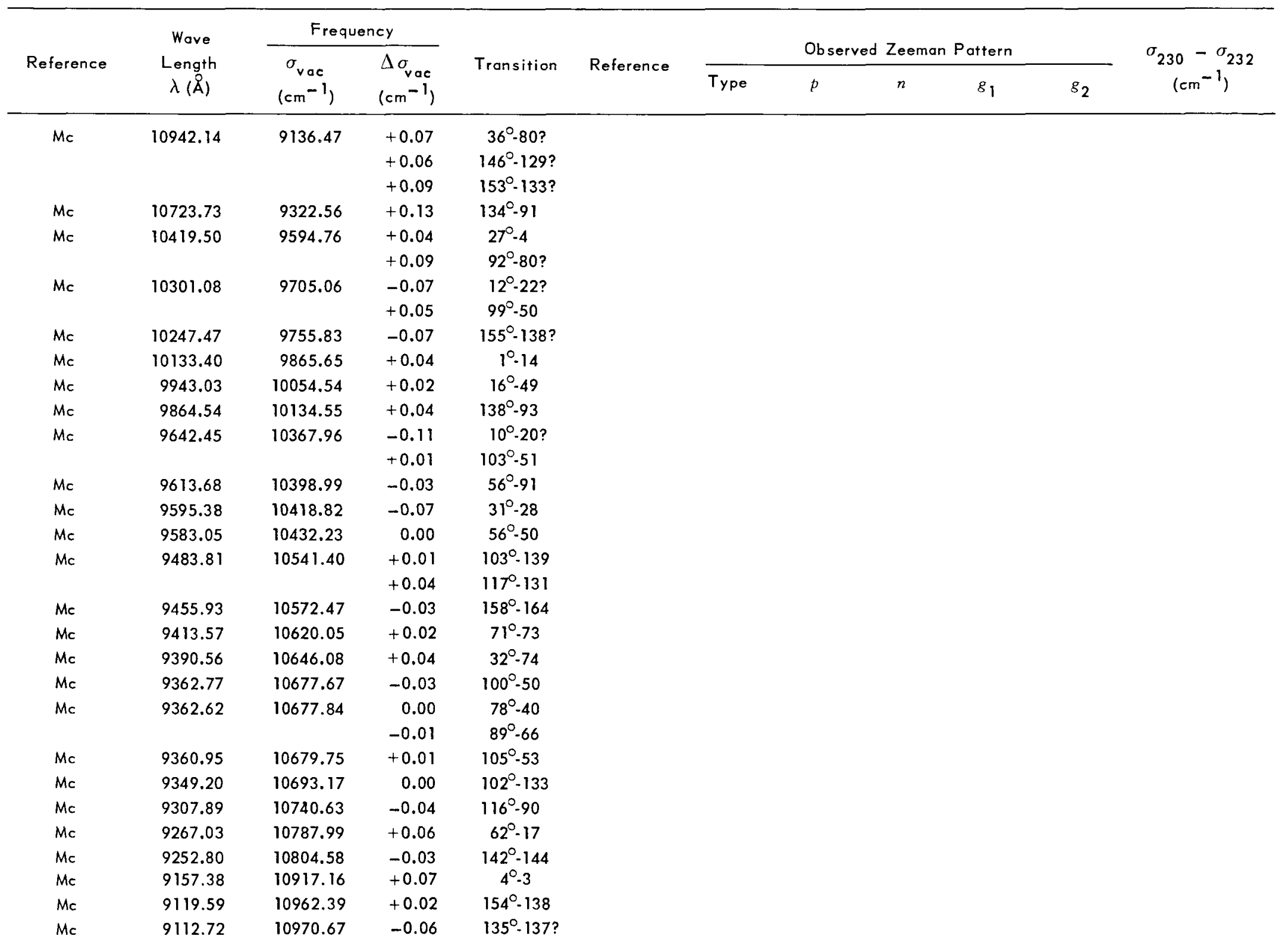


Table 6 (cont inued)

\begin{tabular}{|c|c|c|c|c|c|c|c|c|c|c|c|}
\hline \multirow[b]{2}{*}{ Reference } & \multirow{2}{*}{$\begin{array}{c}\text { Wave } \\
\text { Length } \\
\lambda(\AA)\end{array}$} & \multicolumn{2}{|c|}{ Frequency } & \multirow[b]{2}{*}{ Transition } & \multirow[b]{2}{*}{ Reference } & \multicolumn{5}{|c|}{ Observed Zeeman Pattern } & \multirow[b]{2}{*}{$\begin{array}{c}\sigma_{230}-\sigma_{232} \\
\left(\mathrm{~cm}^{-1}\right)\end{array}$} \\
\hline & & $\begin{array}{c}\sigma_{\text {vac }} \\
\left(\mathrm{cm}^{-1}\right)\end{array}$ & $\begin{array}{l}\Delta \sigma_{\text {vac }} \\
\left(\mathrm{cm}^{-1}\right)\end{array}$ & & & Type & $p$ & $n$ & $g_{1}$ & $g_{2}$ & \\
\hline Me & 9107.56 & 10976.88 & +0.04 & $142^{\circ}-105$ & & & & & & & \\
\hline$M c$ & 9119.59 & 10962.39 & +0.02 & $154^{\circ}-138$ & & & & & & & \\
\hline Me & 9105.20 & 10979.72 & -0.01 & $32^{\circ}-75$ & & & & & & & \\
\hline Me & 9072.23 & 11019.63 & +0.03 & $105^{\circ}-52$ & & & & & & & \\
\hline$M c$ & 9066.07 & 11027.11 & -0.07 & $57^{\circ}-91 ?$ & & & & & & & \\
\hline Me & 9062.57 & 11031.38 & -0.04 & $18^{\circ}-50$ & & & & & & & \\
\hline Mc & 9053.46 & 11042.47 & -0.01 & $17^{\circ}-14$ & & & & & & & \\
\hline Mc & 9040.10 & 11058.79 & 0.00 & $137^{\circ}-133$ & & & & & & & \\
\hline Me & 8957.92 & 11160.23 & 0.00 & $141^{\circ}-94$ & & & & & & & \\
\hline$M c$ & 8949.03 & 11171.33 & -0.04 & $123^{\circ}-141$ & & & & & & & \\
\hline$M c$ & 8842.04 & 11306.51 & +0.02 & $101^{\circ}-91$ & & & & & & & \\
\hline$c$ & 8816.15 & 11339.71 & +0.01 & $101^{\circ}-50$ & & & & & & & \\
\hline$M c$ & 8784.53 & 11380.53 & +0.02 & $17^{\circ}-49$ & & & & & & & \\
\hline Me & 8748.03 & 11428.01 & +0.04 & $99^{\circ} .137 ?$ & & & & & & & \\
\hline Mc & 8709.21 & 11478.95 & -0.02 & $103^{\circ}-140$ & & & & & & & \\
\hline Mc & 8668.10 & 11533.39 & +0.01 & $46^{\circ}-63$ & & & & & & & \\
\hline C & 8629.1 & 11585.5 & 0.0 & $65^{\circ}-92$ & & & & & & & \\
\hline c & 8591.83 & 11635.77 & -0.05 & $156^{\circ}-134$ & & & & & & & \\
\hline C & 8573.10 & 11661.19 & -0.06 & $66^{\circ}-65$ & & & & & & & \\
\hline c & 8450.65 & 11830.16 & 0.00 & $109^{\circ}-93$ & & & & & & & \\
\hline c & 8413.0 & 11883.1 & 0.0 & $19^{\circ}-57$ & & & & & & & \\
\hline c & 8403.79 & 11896.11 & -0.01 & $72^{\circ}-35$ & & & & & & & \\
\hline$M$ & 8275.63 & 12080.35 & -0.03 & $64^{\circ}-51$ & & & & & & & \\
\hline $\mathrm{C}$ & 8252.38 & 12114.39 & 0.00 & $81^{\circ}-132$ & & & & & & & \\
\hline C & 8203.19 & 12187.03 & +0.02 & $144^{\circ}-94$ & & & & & & & \\
\hline \multirow[t]{2}{*}{$c$} & 8166.41 & 12241.92 & +0.02 & $2^{\circ} \cdot 14$ & & & & & & & \\
\hline & & & -0.01 & $82^{\circ}-47$ & & & & & & & \\
\hline \multirow[t]{2}{*}{$\mathrm{C}$} & 8163.11 & 12246.87 & -0.03 & $62^{\circ} \cdot 15$ & & & & & & & \\
\hline & & & +0.01 & $106^{\circ}-51$ & & & & & & & \\
\hline M & 8143.15 & 12276.89 & -0.07 & $5^{\circ}-3 ?$ & & & & & & & \\
\hline c & 8139.88 & 12281.82 & +0.06 & $108^{\circ}-53$ & & & & & & & \\
\hline c & 8048.26 & 12421.65 & -0.03 & $82^{\circ}-96$ & & & & & & & \\
\hline c & 8036.87 & 12439.23 & +0.02 & $102^{\circ}-61$ & & & & & & & \\
\hline
\end{tabular}


Table 6 (cont inued)

\begin{tabular}{|c|c|c|c|c|c|c|c|c|c|c|c|}
\hline \multirow{3}{*}{ Reference } & \multirow{3}{*}{$\begin{array}{c}\text { Wave } \\
\text { Length } \\
\lambda(\AA)\end{array}$} & \multicolumn{2}{|c|}{ Frequency } & \multirow{3}{*}{ Transition } & \multirow{3}{*}{ Reference } & \multirow{2}{*}{\multicolumn{5}{|c|}{ Observed Zeeman Pattern }} & \multirow{3}{*}{$\begin{array}{c}\sigma_{230}-\sigma_{232} \\
\left(\mathrm{~cm}^{-1}\right)\end{array}$} \\
\hline & & \multirow{2}{*}{$\begin{array}{c}\sigma_{\mathrm{Vac}} \\
\left(\mathrm{cm}^{-1}\right)\end{array}$} & \multirow{2}{*}{$\begin{array}{l}\Delta \sigma_{\mathrm{vac}} \\
\left(\mathrm{cm}^{-1}\right)\end{array}$} & & & & & & & & \\
\hline & & & & & & Type & $p$ & $n$ & $g_{1}$ & $g_{2}$ & \\
\hline$M$ & 8032.48 & 12446.04 & 0.00 & $43^{\circ}-58$ & & & & & & & \\
\hline$c$ & 8015.64 & 12472.18 & 0.00 & $55^{\circ}-13$ & & & & & & & \\
\hline$c$ & 8015.65 & 12472.14 & \pm 0.04 & $50^{\circ} \cdot 13$ & & & & & & & \\
\hline$c$ & 7941.77 & 12588.21 & +0.05 & $129^{\circ}-156$ & & & & & & & \\
\hline$c$ & 7928.97 & 12608.52 & +0.05 & $102^{\circ}-139$ & & & & & & & \\
\hline$c$ & 7900.35 & 12654.21 & +0.17 & $68^{\circ}-18 ?$ & & & & & & & \\
\hline$c$ & 7896.46 & 12660.42 & +0.02 & $2^{\circ}-20$ & & & & & & & \\
\hline$c$ & 7893.66 & 12664.91 & +0.06 & $69^{\circ}-71$ & & & & & & & \\
\hline$c$ & 7849.60 & 12736.02 & +0.08 & $100^{\circ}-138$ & & & & & & & \\
\hline$c$ & 7834.45 & 12760.63 & 0.00 & $98^{\circ}-137$ & & & & & & & \\
\hline$K$ & 7808.04 & 12803.79 & -0.01 & $148^{\circ}-139$ & & & & & & & \\
\hline$M$ & 7798.33 & 12819.74 & -0.03 & $31^{\circ} \cdot 33$ & & & & & & & \\
\hline$c$ & 7676.19 & 13023.74 & +0.06 & $56^{\circ}-49$ & & & & & & & \\
\hline$c$ & 7632.14 & 13098.88 & -0.04 & $129^{\circ}-155$ & & & & & & & \\
\hline \multirow[t]{2}{*}{$c$} & 7624.27 & 13112.41 & -0.03 & $66^{\circ}-53$ & & & & & & & \\
\hline & & & -0.06 & $78^{\circ}-90 ?$ & & & & & & & \\
\hline C & 7528.46 & 13279.28 & +0.02 & $65^{\circ}-16$ & & & & & & & \\
\hline $\mathrm{s}$ & 7525.52 & 13284.46 & -0.10 & $109^{\circ}-53$ & $\mathrm{~S}$ & & $(0)$ & 0.88 & & & \\
\hline C & 7490.09 & 13347.28 & -0.06 & $60^{\circ}-50$ & & & & & & & \\
\hline$c$ & 7449.80 & 13419.49 & +0.02 & $90^{\circ}-101$ & & & & & & & \\
\hline$M$ & 7447.84 & 13423.02 & 0.00 & $41^{\circ}-48$ & & & & & & & \\
\hline$c$ & 7437.72 & 13441.29 & +0.03 & $156^{\circ}-145$ & & & & & & & \\
\hline$M$ & 7430.27 & 13454.76 & +0.10 & $45^{\circ} \cdot 60$ & & & & & & & \\
\hline$C$ & 7411.75 & 13488.40 & +0.06 & $64^{\circ}-65$ & & & & & & & \\
\hline$M$ & 7393.433 & 13521.80 & -0.04 & $158^{\circ}-135$ & & & & & & & \\
\hline C & 7380.20 & 13546.04 & -0.01 & $102^{\circ}-140$ & & & & & & & \\
\hline$C$ & 7358.37 & 13586.23 & +0.01 & $17^{\circ} \cdot 56$ & & & & & & & \\
\hline$c$ & 7338.60 & 13622.82 & -0.05 & $18^{\circ}-49$ & & & & & & & \\
\hline$c$ & 7292.70 & 13708.56 & -0.07 & $99^{\circ}-138$ & & & & & & & \\
\hline$M$ & 7288.98 & 13715.57 & -0.03 & $104^{\circ} \cdot 133$ & & & & & & & \\
\hline
\end{tabular}


Table 6 (cont inved)

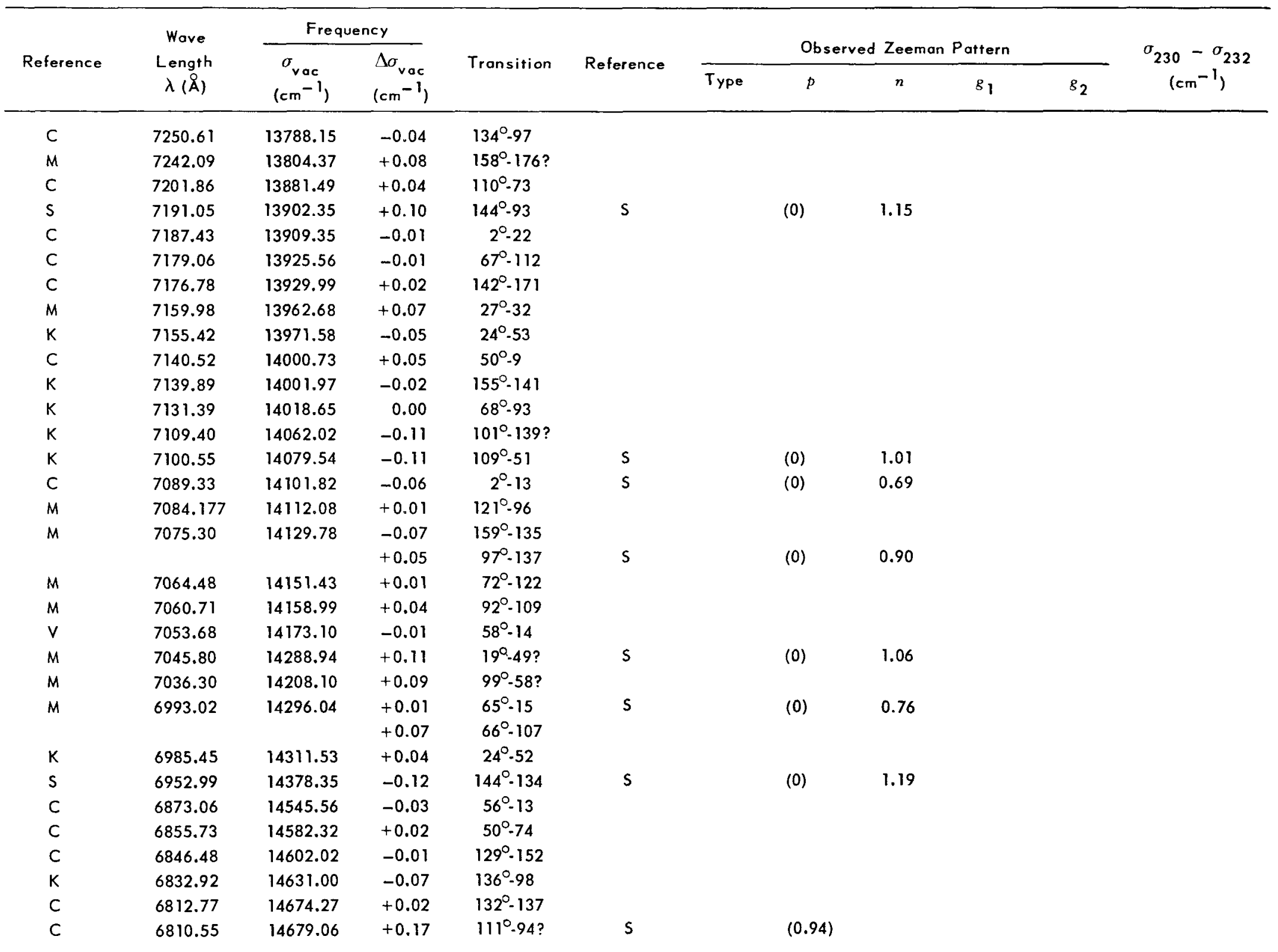


Table 6 (continued)

\begin{tabular}{|c|c|c|c|c|c|c|c|c|c|c|c|}
\hline \multirow{3}{*}{ Reference } & \multirow{3}{*}{$\begin{array}{c}\text { Wave } \\
\text { Length } \\
\lambda(\AA)\end{array}$} & \multicolumn{2}{|c|}{ Frequency } & \multirow{3}{*}{ Transition } & \multirow{3}{*}{ Reference } & \multirow{2}{*}{\multicolumn{5}{|c|}{ Observed Zeeman Pottern }} & \multirow{3}{*}{$\begin{array}{c}\sigma_{230}-\sigma_{232} \\
\left(\mathrm{~cm}^{-1}\right)\end{array}$} \\
\hline & & \multirow{2}{*}{$\begin{array}{c}\sigma_{\mathrm{vac}} \\
\left(\mathrm{cm}^{-1}\right)\end{array}$} & \multirow{2}{*}{$\begin{array}{l}\Delta \sigma_{\text {vac }} \\
\left(\mathrm{cm}^{-1}\right)\end{array}$} & & & & & & & & \\
\hline & & & & & & Type & $p$ & $n$ & $g_{1}$ & $g_{2}$ & \\
\hline K & 6804.75 & 14691.57 & -0.03 & $17^{\circ}-57$ & & & & & & & \\
\hline $\mathrm{K}$ & 6770.13 & 14766.70 & -0.02 & $24^{\circ}-51$ & & & & & & & \\
\hline$c$ & 6765.70 & 14776.37 & +0.07 & $138^{\circ}-133$ & & & & & & & \\
\hline $\mathrm{C}$ & 6760.87 & 14786.92 & +0.05 & $45^{\circ}-21$ & & & & & & & \\
\hline $\mathrm{C}$ & 6758.26 & 14792.63 & +0.01 & $74^{\circ}-88$ & & & & & & & \\
\hline C & 6733.78 & 14846.41 & -0.09 & $58^{\circ}-99$ & & & & & & & \\
\hline $\mathrm{C}$ & 6730.52 & 14853.60 & -0.08 & $143^{\circ} \cdot 115$ & & & & & & & \\
\hline c & 6727.44 & 14860.40 & -0.04 & $103^{\circ}-50$ & & & & & & & \\
\hline $\mathrm{C}$ & 6706.86 & 14906.00 & +0.01 & $113^{\circ}-129$ & & & & & & & \\
\hline KC & 6704.07 & 14912.20 & +0.02 & $24^{\circ}-17$ & & & & & & & \\
\hline & & & -0.01 & $16^{\circ}-56$ & & & & & & & \\
\hline$c$ & 6700.51 & 14920.13 & +0.02 & $140^{\circ}-170$ & & & & & & & \\
\hline $\mathrm{C}$ & 6697.75 & 14926.27 & +0.02 & $62^{\circ}-65$ & & & & & & & \\
\hline $\mathrm{C}$ & 6692.69 & 14937.56 & +0.05 & $22^{\circ}-50$ & & & & & & & \\
\hline$c$ & 6687.58 & 14948.97 & -0.04 & $31^{\circ}-80$ & & & & & & & \\
\hline$M$ & 6658.681 & 15013.85 & +0.19 & $26^{\circ}-53 ?$ & & & & & & & \\
\hline$C, K$ & 6648.51 & 15036.82 & +0.09 & $1^{\circ}-20$ & & & & & & & \\
\hline$M$ & 6646.52 & 15041.32 & +0.02 & $98^{\circ}-138$ & & & & & & & \\
\hline$M, S$ & 6644.645 & 15045.57 & -0.07 & $144^{\circ} .92$ & $S$ & & $(0)$ & 1.57 & & & \\
\hline $\mathrm{K}$ & 6637.48 & 15061.81 & +0.01 & $148^{\circ} .97$ & & & & & & & \\
\hline $\mathrm{K}$ & 6633.42 & 15071.03 & +0.06 & $25^{\circ}-52$ & & & & & & & \\
\hline $\mathrm{K}$ & 6619.88 & 15101.85 & +0.10 & $62^{\circ}-91 ?$ & $S$ & & $(0)$ & 1.85 & & & \\
\hline $\mathrm{K}$ & 6616.99 & 15108.45 & +0.14 & $98^{\circ}-57 ?$ & & & & & & & \\
\hline$M$ & 6616.89 & 15108.68 & -0.14 & $17^{\circ} \cdot 22 ?$ & & & & & & & \\
\hline$M$ & 6605.432 & 15134.89 & -0.07 & $62^{\circ}-50$ & $\mathrm{~S}$ & & $(0.55)$ & 0.93 & & & \\
\hline $\mathrm{K}$ & 6593.58 & 15162.09 & +0.05 & $68^{\circ}-92$ & & & & & & & \\
\hline$M$ & 6577.73 & 15198.60 & -0.11 & $140^{\circ}-149 ?$ & & & & & & & \\
\hline $\mathrm{K}$ & 6572.86 & 15209.89 & +0.02 & $136^{\circ} \cdot 140$ & & & & & & & \\
\hline$M$ & 6560.02 & 15239.66 & +0.08 & $135^{\circ} \cdot 139$ & $M$ & 6 & $(1.063)$ & 0.913 & 0.795 & 1.032 & \\
\hline$K$ & 654458 & 15275.61 & +0.11 & $60^{\circ}-101$ & & & & & & & \\
\hline$c$ & 6540.11 & 15286.05 & +0.06 & $26^{\circ}-75$ & & & & & & & \\
\hline $\mathrm{K}$ & 6532.23 & 15304.49 & +0.06 & $137^{\circ} \cdot 100$ & & & & & & & \\
\hline$K$ & 6522.04 & 15328.40 & +0.02 & $56^{\circ}-98$ & & & & & & & \\
\hline
\end{tabular}


Table 6 (continued)

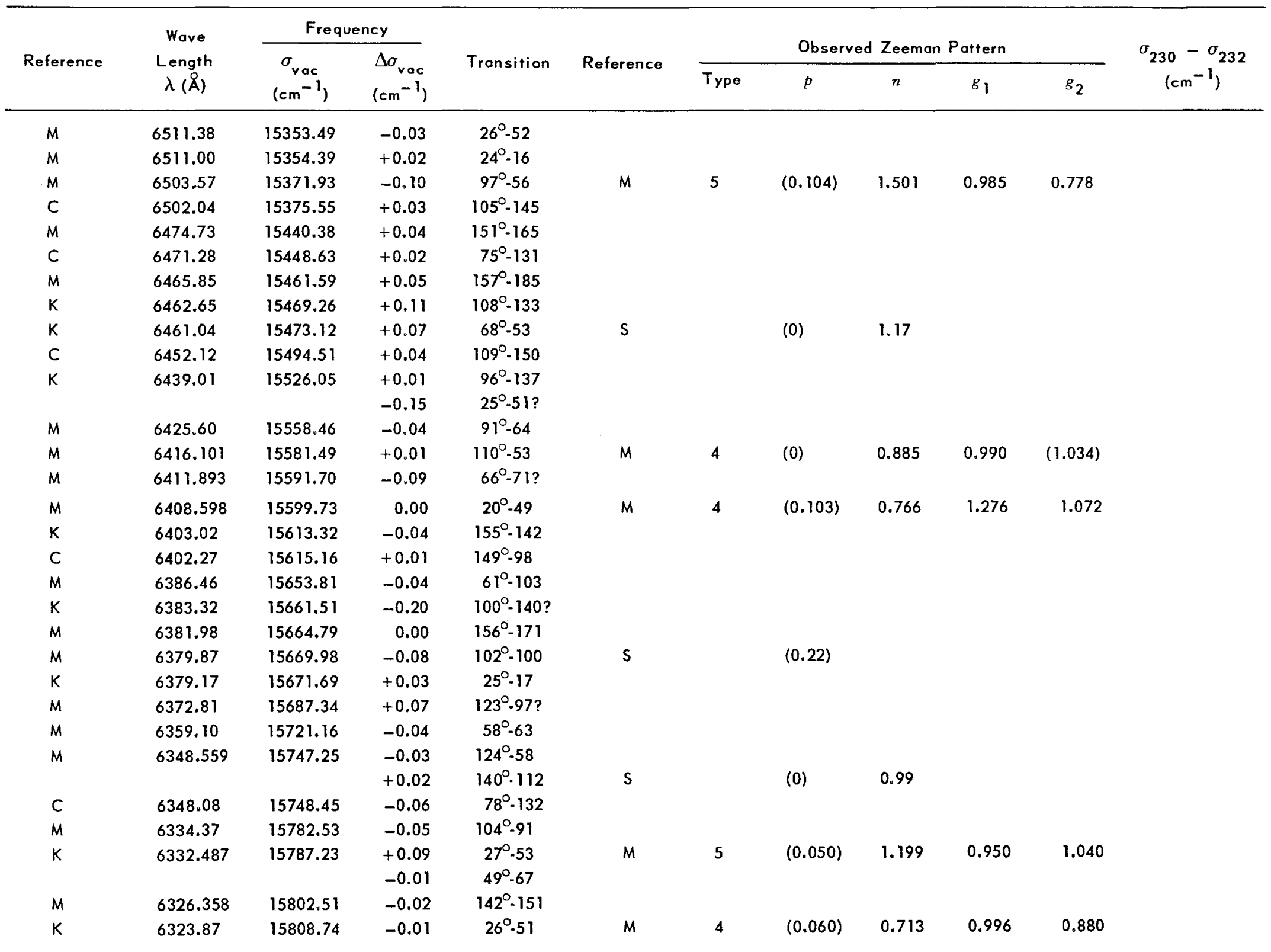


Table 6 (continued)

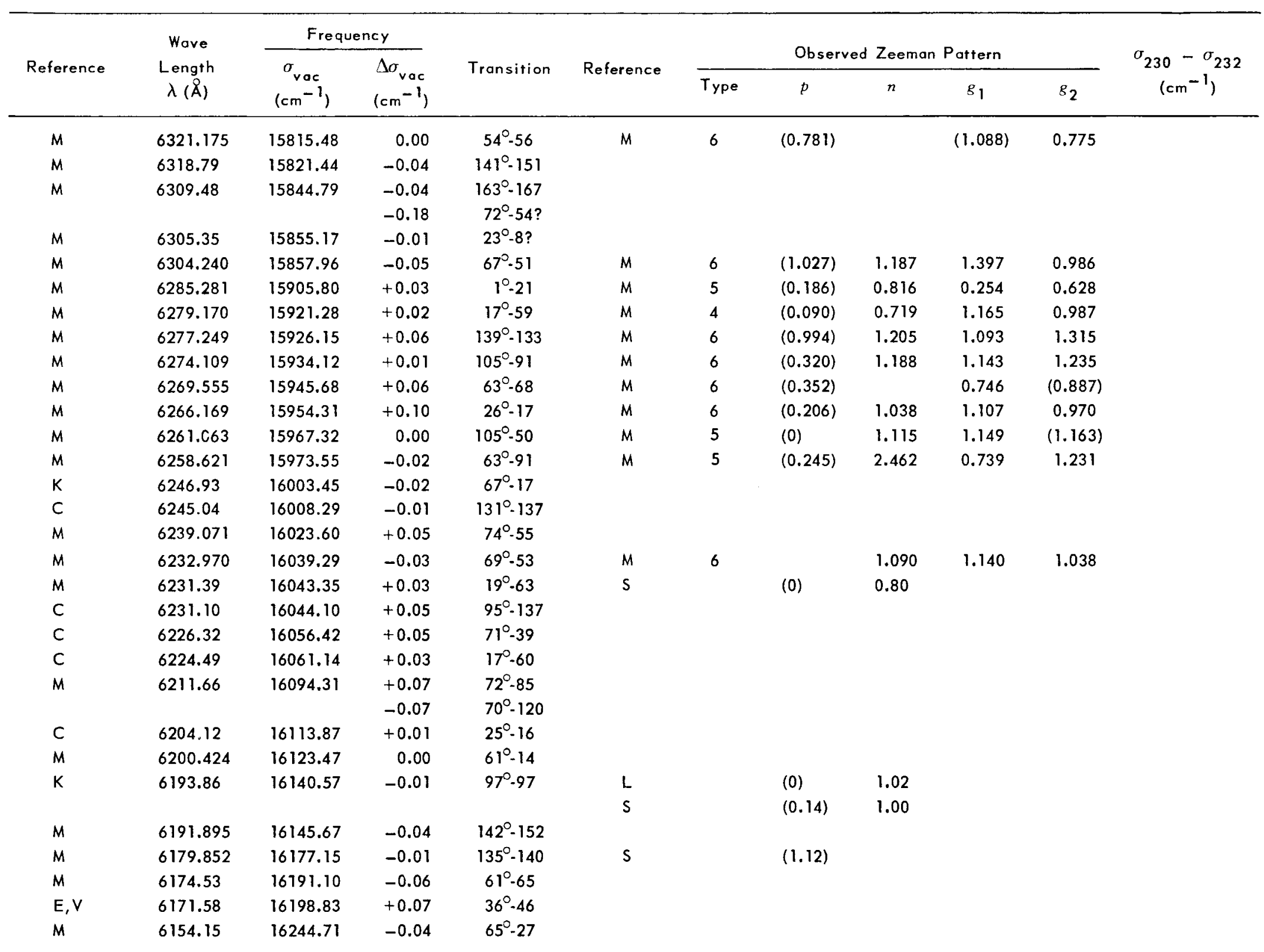




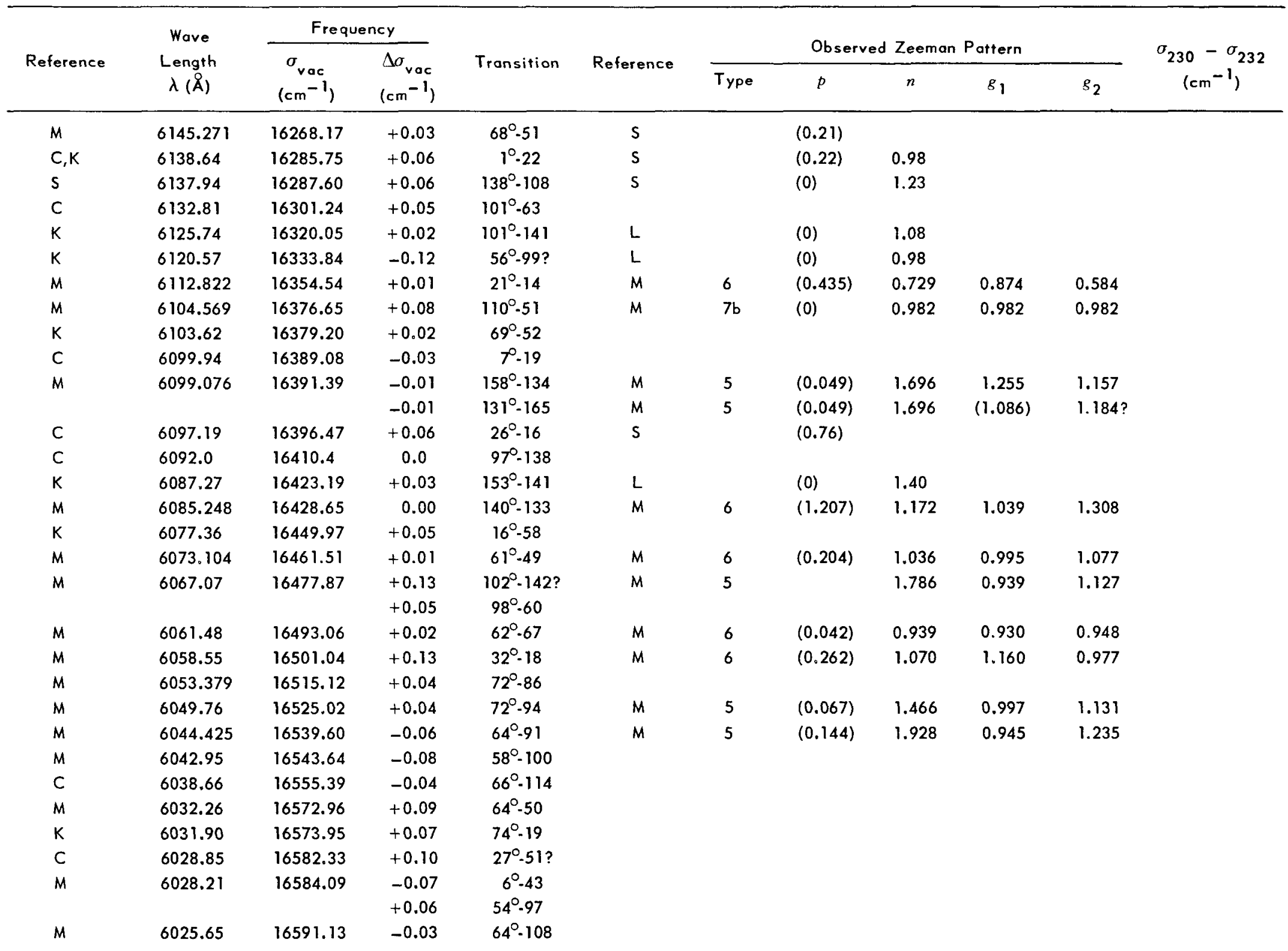


Table 6 (cont inued)

\begin{tabular}{|c|c|c|c|c|c|c|c|c|c|c|c|}
\hline \multirow{3}{*}{ Reference } & \multirow{3}{*}{$\begin{array}{c}\text { Wave } \\
\text { Length } \\
\lambda(\AA)\end{array}$} & \multicolumn{2}{|c|}{ Frequency } & \multirow{3}{*}{ Transition } & \multirow{3}{*}{ Reference } & \multirow{2}{*}{\multicolumn{5}{|c|}{ Observed Zeeman Pattern }} & \multirow{3}{*}{$\begin{array}{c}\sigma_{230}-\sigma_{232} \\
\left(\mathrm{~cm}^{-1}\right)\end{array}$} \\
\hline & & \multirow{2}{*}{$\begin{array}{c}\sigma_{\mathrm{vac}} \\
\left(\mathrm{cm}^{-1}\right)\end{array}$} & \multirow{2}{*}{$\begin{array}{l}\Delta \sigma_{\text {voc }} \\
\left(\mathrm{cm}^{-1}\right)\end{array}$} & & & & & & & & \\
\hline & & & & & & Type & $p$ & $n$ & $g_{1}$ & $g_{2}$ & \\
\hline$M$ & 6021.035 & 16603.84 & -0.10 & $135^{\circ}-99 ?$ & & & & & & & \\
\hline$M$ & 6018.99 & 16609.49 & +0.02 & $18^{\circ}-63$ & $M$ & 4 & $(0.209)$ & 0.329 & 1.370 & 0.952 & \\
\hline C & 6017.8 & 16612.8 & 0.0 & $106^{\circ}-69 ?$ & & & & & & & \\
\hline$s$ & 6011.35 & 16630.60 & +0.03 & $62^{\circ}-106$ & $\mathrm{~S}$ & & $(0)$ & 1.04 & & & \\
\hline C & 6005.16 & 16647.75 & +0.11 & $65^{\circ} .70$ & & & & & & & \\
\hline $\mathrm{K}$ & 5991.74 & 16685.03 & -0.07 & $132^{\circ}-97$ & & & & & & & \\
\hline C & 5989.8 & 16690.4 & -0.1 & $22^{\circ}-67$ & & & & & & & \\
\hline$M$ & 5989.035 & 16692.57 & +0.01 & $21^{\circ}-49$ & $M$ & 5 & $(0.100)$ & 1.374 & 0.876 & 1.075 & \\
\hline $\mathrm{s}$ & 5983.99 & 16706.64 & -0.09 & $108^{\circ}-112$ & $M$ & 6 & $(0.558)$ & 1.162 & 1.242 & 1.083 & \\
\hline$K$ & 5983.42 & 16708.23 & -0.06 & $163^{\circ}-168$ & & & & & & & \\
\hline$K$ & 5981.91 & 16712.45 & -0.08 & $70^{\circ}-92$ & & & & & & & \\
\hline$M$ & 5981.79 & 16712.78 & +0.11 & $91^{\circ}-100 ?$ & & & & & & & \\
\hline$K$ & 5981.39 & 16713.90 & +0.16 & $56^{\circ}-62 ?$ & & & & & & & \\
\hline & & & +0.03 & $60^{\circ}-65$ & & & & & & & \\
\hline$M$ & 5976.439 & 16727.74 & +0.05 & $27^{\circ}-17$ & & & & & & & \\
\hline$M$ & 5961.973 & 16768.34 & 0.00 & $96^{\circ}-56$ & $M$ & 5 & $(0.199)$ & 0.961 & 1.170 & 0.771 & \\
\hline$M$ & 5960.376 & 16772.82 & -0.04 & $109^{\circ}-115$ & & & & & & & \\
\hline V & 5958.1 & 16779.2 & -0.1 & $64^{\circ}-69$ & & & & & & & \\
\hline$M$ & 5948.59 & 16806.06 & -0.13 & $4^{\circ}-17 ?$ & & & & & & & \\
\hline$M$ & 5944.58 & 16817.40 & -0.04 & $62^{\circ}-68$ & & & & & & & \\
\hline$K$ & 5943.75 & 16819.74 & -0.09 & $154^{\circ} \cdot 142$ & & & & & & & \\
\hline$M$ & 5938.582 & 16834.38 & -0.03 & $69^{\circ}-51$ & $M$ & 6 & $(0.382)$ & 1.063 & 1.141 & 0.988 & \\
\hline$K$ & 5931.02 & 16855.85 & +0.05 & $68^{\circ} \cdot 16$ & & & & & & & \\
\hline$M$ & 5929.487 & 16860.19 & -0.03 & $98^{\circ}-6 \mathrm{l}$ & $\mathrm{s}$ & & $(0)$ & 0.63 & & & \\
\hline$M$ & 5927.97 & 16864.51 & -0.01 & $67^{\circ} \cdot 79$ & & & & & & & \\
\hline$M$ & 5925.894 & 16870.42 & -0.02 & $111^{\circ}-134$ & $M$ & 4 & $(0.114)$ & 0.350 & 1.382 & 1.153 & \\
\hline C & 5922.82 & 16879.18 & +0.05 & $74^{\circ}-44$ & & & & & & & \\
\hline$M$ & 5914.395 & 16903.23 & 0.00 & $53^{\circ}-56$ & $M$ & 6 & $(0.438)$ & 0.689 & 0.600 & 0.775 & \\
\hline$M$ & 5906.271 & 16926.48 & -0.08 & $58^{\circ}-23$ & $M$ & 5 & $(0.101)$ & 1.385 & 1.066 & 0.856 & \\
\hline$c$ & 5895.25 & 16958.12 & -0.04 & $54^{\circ}-21$ & & & & & & & \\
\hline$M$ & 5893.498 & 16963.15 & -0.04 & $100^{\circ}-63$ & 5 & & $(0)$ & 0.77 & & & \\
\hline$M$ & 5886.32 & 16983.83 & +0.06 & $134^{\circ} \cdot 140$ & & & & & & & \\
\hline$M$ & 5885.714 & 16985.60 & +0.07 & $31^{\circ}-83$ & & & & & & & \\
\hline
\end{tabular}




\begin{tabular}{|c|c|c|c|c|c|c|c|c|c|c|c|}
\hline \multirow{3}{*}{ Reference } & \multirow{3}{*}{$\begin{array}{c}\text { Wove } \\
\text { Length } \\
\lambda(\AA)\end{array}$} & \multicolumn{2}{|c|}{ Frequency } & \multirow{3}{*}{ Transition } & \multirow{3}{*}{ Reference } & \multirow{2}{*}{\multicolumn{5}{|c|}{ Observed Zeeman Pottern }} & \multirow{3}{*}{$\begin{array}{c}\sigma_{230}-\sigma_{232} \\
\left(\mathrm{~cm}^{-1}\right)\end{array}$} \\
\hline & & $\sigma_{\text {vac }}$ & $\Delta \sigma_{\text {vac }}$ & & & & & & & & \\
\hline & & $\left(\mathrm{cm}^{-1}\right)$ & $\left(\mathrm{cm}^{-1}\right)$ & & & Type & $p$ & $n$ & $g_{1}$ & $g_{2}$ & \\
\hline M & 5881.44 & 16997.93 & -0.02 & $137^{\circ}-143$ & & & & & & & \\
\hline K & 5879.48 & 17003.61 & -0.02 & $34^{\circ}-3$ & & & & & & & \\
\hline M & 5872.559 & 17023.63 & +0.04 & $70^{\circ}-53$ & M & 6 & $(0.349)$ & 1.177 & 1.107 & 1.247 & \\
\hline \multirow[t]{2}{*}{ M } & 5870.572 & 17029.39 & -0.09 & $98^{\circ}-139$ & L & & (0) & 1.67 & & & \\
\hline & & & & & M & 5 & $(0.063)$ & 1.724 & 0.896 & 1.080 & \\
\hline \multirow[t]{2}{*}{$M$} & 5859.688 & 17061.02 & -0.02 & $107^{\circ}-91$ & & & & & & & \\
\hline & & & -0.14 & $99^{\circ}-99$ & L & & $(0.19)$ & 1.20 & & & \\
\hline C & 5858.69 & 17063.94 & -0.01 & $7^{\circ}-44$ & & & & & & & \\
\hline C & 5855.47 & 17073.32 & -0.02 & $59^{\circ} \cdot 102$ & & & & & & & \\
\hline K & 5852.16 & 17082.98 & +0.05 & $60^{\circ}-66$ & & & & & & & \\
\hline$M$ & 5838.951 & 17121.63 & -0.01 & $20^{\circ}-13$ & M & 6 & $(0.958)$ & 0.956 & 1.278 & 0.639 & \\
\hline K & 5835.85 & 17130.72 & +0.09 & $25^{\circ}-15$ & & & & & & & \\
\hline c & 5822.50 & 17170.00 & +0.11 & $27^{\circ}-16 ?$ & & & & & & & \\
\hline M & 5820.32 & 17176.43 & +0.05 & $58^{\circ}-102$ & & & & & & & \\
\hline $\mathrm{k}$ & 5820.14 & 17176.96 & +0.09 & $53^{\circ}-20$ & & & & & & & \\
\hline M & 5817.69 & 17184.20 & +0.11 & $65^{\circ}-50$ & & & & & & & \\
\hline M & 5815.432 & 17190.87 & -0.06 & $22^{\circ}-14$ & M & 6 & $(0.456)$ & 0.738 & 0.887 & 0.586 & \\
\hline k & 5809.38 & 17208.78 & +0.12 & $56^{\circ}-63 ?$ & & & & & & & \\
\hline M & 5808.06 & 17212.68 & -0.01 & $139^{\circ}-174$ & & & & & & & \\
\hline K & 5796.40 & 17247.32 & +0.07 & $16^{\circ} \cdot 59$ & M & 5 & $(0.072)$ & 1.225 & 0.843 & 0.995 & \\
\hline M & 5795.99 & 17248.54 & +0.15 & $4^{\circ} \cdot 16 ?$ & & & & & & & \\
\hline$c$ & 5794.4 & 17253.3 & 0.0 & $140^{\circ}-152 ?$ & & & & & & & \\
\hline$k$ & 5790.12 & 17266.02 & +0.12 & $60^{\circ}-105 ?$ & & & & & & & \\
\hline c & 5788.2 & 17271.8 & 0.0 & $24^{\circ} \cdot 77$ & & & & & & & \\
\hline K & 5786.68 & 17276.29 & +0.03 & $\Upsilon^{\circ}-3$ & & & & & & & \\
\hline $\mathrm{s}$ & 5778.08 & 17301.99 & +0.03 & $138^{\circ}-111$ & $s$ & & $(0)$ & 0.99 & & & \\
\hline M & 5769.67 & 17327.21 & -0.05 & $106^{\circ}-150$ & & & & & & & \\
\hline \multirow[t]{2}{*}{ K } & 5766.10 & 17337.95 & +0.05 & $151^{\circ}-138$ & & & & & & & \\
\hline & & & -0.13 & $54^{\circ}-22 ?$ & & & & & & & \\
\hline $\mathrm{K}$ & 5758.92 & 17359.56 & +0.09 & $27^{\circ}-2$ & & & & & & & \\
\hline \multirow[t]{2}{*}{$K, C$} & 5757.62 & 17363.48 & +0.03 & $70^{\circ}-52$ & & & & & & & \\
\hline & & & -0.09 & $102^{\circ}-143$ & & & & & & & \\
\hline C & 5754.6 & 17372.6 & 0.0 & $102^{\circ}-104$ & & & & & & & \\
\hline
\end{tabular}


Table 6 (cont inued)

\begin{tabular}{|c|c|c|c|c|c|c|c|c|c|c|c|}
\hline \multirow{3}{*}{ Reference } & \multirow{3}{*}{$\begin{array}{c}\text { Wave } \\
\text { Length } \\
\lambda(\AA)\end{array}$} & \multicolumn{2}{|c|}{ Frequency } & \multirow{3}{*}{ Transition } & \multirow{3}{*}{ Reference } & \multirow{2}{*}{\multicolumn{5}{|c|}{ Observed Zeeman Pattern }} & \multirow{3}{*}{$\begin{array}{c}\sigma_{230}-\sigma_{232} \\
\left(\mathrm{~cm}^{-1}\right)\end{array}$} \\
\hline & & \multirow{2}{*}{$\begin{array}{c}\sigma_{v a c} \\
\left(\mathrm{~cm}^{-1}\right)\end{array}$} & \multirow{2}{*}{$\begin{array}{c}\Delta \sigma_{\mathrm{vac}} \\
\left(\mathrm{cm}^{-1}\right)\end{array}$} & & & & & & & & \\
\hline & & & & & & Type & $p$ & $n$ & $g_{1}$ & $g_{2}$ & \\
\hline$K$ & 5750.27 & 17385.68 & +0.05 & $133^{\circ}-98$ & & & & & & & \\
\hline \multirow[t]{2}{*}{$M$} & 5749.379 & 17388.37 & -0.01 & $62^{\circ} \cdot 14$ & $M$ & 5 & $(0.172)$ & 1.450 & 0.936 & 0.592 & \\
\hline & & & -0.13 & $71^{\circ}-93 ?$ & & & & & & & \\
\hline \multirow[t]{2}{*}{$M$} & 5745.665 & 17399.60 & +0.02 & $138^{\circ}-112$ & $M$ & 4 & $(0.064)$ & 0.513 & 0.961 & 1.089 & \\
\hline & & & & & $\mathrm{s}$ & & $(0)$ & 0.62 & & & \\
\hline \multirow[t]{2}{*}{$M, K$} & 5742.085 & 17410.46 & -0.09 & $134^{\circ}-99$ & $L$ & & $(0)$ & 1.10 & & & \\
\hline & & & -0.02 & $157^{\circ}-133$ & & & & & & & \\
\hline$M$ & 5741.362 & 17413.26 & +0.08 & $26^{\circ} \cdot 15$ & $M$ & 6 & $(0.452)$ & 0.924 & 1.078 & 0.774 & \\
\hline \multirow[t]{3}{*}{$M$} & 5738.26 & 17422.06 & -0.01 & $69^{\circ}-16$ & & & & & & & \\
\hline & & & +0.07 & $20^{\circ} \cdot 66$ & & & & & & & \\
\hline & & & 0.00 & $63^{\circ}-27$ & & & & & & & \\
\hline$M$ & 5736.03 & 17428.84 & +0.08 & $36^{\circ}-55$ & & & & & & & \\
\hline$K$ & 5732.05 & 17440.94 & -0.02 & $99^{\circ} \cdot 62$ & & & & & & & \\
\hline$K$ & 5727.69 & 17454.22 & -0.04 & $137^{\circ}=105$ & & & & & & & \\
\hline$M$ & 5725.031 & 17462.32 & -0.12 & $67^{\circ}-15 ?$ & & & & & & & \\
\hline$M$ & 5724.35 & 17464.40 & -0.07 & $34^{\circ}-45$ & & & & & & & \\
\hline$C$ & 5724.21 & 17464.83 & +0.03 & $144^{\circ}-119$ & & & & & & & \\
\hline$M$ & 5712.54 & 17500.50 & +0.03 & $33^{\circ}-18$ & & & & & & & \\
\hline$M$ & 5707.094 & 17517.20 & -0.06 & $141^{\circ} \cdot 133$ & $M$ & 6 & $(0.565)$ & 1.256 & 1.189 & 1.315 & \\
\hline$M$ & 5703.96 & 17526.83 & -0.06 & $27^{\circ} \cdot 67$ & & & & & & & \\
\hline C & 5703.32 & 17528.80 & -0.16 & $22^{\circ}-49 ?$ & & & & & & & \\
\hline \multirow[t]{2}{*}{ M } & 5700.909 & 17536.20 & -0.01 & $142^{\circ}-133$ & & & & & & & \\
\hline & & & +0.07 & $108^{\circ}-91$ & L & & (0) & 1.22 & & & \\
\hline$K$ & 5700.696 & 17536.86 & -0.03 & $96^{\circ} \cdot 97$ & $\mathrm{~s}$ & & $(0.51)$ & & & & \\
\hline$M$ & 5700.451 & 17537.61 & 0.00 & $111^{\circ}-92$ & & & & & & & \\
\hline$c$ & 5698.55 & 17543.47 & +0.15 & $32^{\circ}-41 ?$ & & & & & & & \\
\hline $\mathrm{K}$ & 5692.87 & 17560.97 & 0.00 & $62^{\circ}-107$ & & & & & & & \\
\hline $\mathrm{K}$ & 5690.17 & 17569.31 & -0.03 & $108^{\circ} \cdot 50$ & & & & & & & \\
\hline$c$ & 5683.4 & 17590.2 & 0.0 & $89^{\circ}-21 ?$ & & & & & & & \\
\hline \multirow[t]{3}{*}{$M$} & 5678.93 & 17604.08 & 0.00 & $33^{\circ}-90$ & & & & & & & \\
\hline & & & -0.11 & $58^{\circ}-103 ?$ & & & & & & & \\
\hline & & & +0.11 & $74^{\circ} \cdot 46 ?$ & & & & & & & \\
\hline
\end{tabular}


Table 6 (cont inued)

\begin{tabular}{|c|c|c|c|c|c|c|c|c|c|c|c|}
\hline \multirow{3}{*}{ Reference } & \multirow{3}{*}{$\begin{array}{c}\text { Wave } \\
\text { Length } \\
\lambda(\AA)\end{array}$} & \multicolumn{2}{|c|}{ Frequency } & \multirow{3}{*}{ Transition } & \multirow{3}{*}{ Reference } & \multirow{2}{*}{\multicolumn{5}{|c|}{ Observed Zeeman Pattern }} & \multirow{3}{*}{$\begin{array}{c}\sigma_{230}-\sigma_{232} \\
\left(\mathrm{~cm}^{-1}\right)\end{array}$} \\
\hline & & \multirow{2}{*}{$\begin{array}{c}\sigma_{\text {vac }} \\
\left(\mathrm{cm}^{-1}\right)\end{array}$} & \multirow{2}{*}{$\begin{array}{l}\Delta \sigma_{\text {vac }} \\
\left(\mathrm{cm}^{-1}\right)\end{array}$} & & & & & & & & \\
\hline & & & & & & Type & $p$ & $n$ & $g_{1}$ & $g_{2}$ & \\
\hline C & 5671.0 & 17628.7 & +0.1 & $107^{\circ}-113 ?$ & & & & & & & \\
\hline $\mathrm{C}$ & 5660.0 & 17663.0 & +0.1 & $127^{\circ}-58 ?$ & & & & & & & \\
\hline$M$ & 5657.926 & 17669.43 & -0.08 & $77^{\circ}-48$ & & & & & & & \\
\hline$k$ & 5657.21 & 17671.67 & -0.11 & $53^{\circ}-97 ?$ & & & & & & & \\
\hline$M$ & 5652.902 & 17685.13 & +0.03 & $155^{\circ}-145$ & 5 & & (0) & 1.22 & & & \\
\hline$M$ & 5650.32 & 17693.20 & +0.01 & $94^{\circ}-96$ & & & & & & & \\
\hline K & 5648.86 & 17697.78 & -0.11 & $58^{\circ}-64$ & & & & & & & \\
\hline$M$ & 5645.888 & 17707.10 & +0.03 & $97^{\circ}-59$ & $M$ & $7 b$ & (0) & 0.978 & 0.978 & 0.978 & \\
\hline K & 5641.23 & 17721.72 & +0.04 & $73^{\circ}-54$ & & & & & & & \\
\hline$M$ & 5639.733 & 17726.43 & +0.02 & $62^{\circ}-49$ & $M$ & 6 & $(0.359)$ & 1.004 & 0.931 & 1.076 & \\
\hline$K$ & 5633.65 & 17745.56 & -0.06 & $112^{\circ}-135$ & & & & & & & \\
\hline$M$ & 5626.70 & 17767.49 & +0.13 & $164^{\circ}-171 ?$ & $S$ & & $(0)$ & 1.30 & & & \\
\hline$M$ & 5620.92 & 17785.74 & +0.03 & $100^{\circ}-100$ & & & & & & & \\
\hline$c$ & 5619.95 & 17788.83 & +0.04 & $7^{\circ}-46$ & & & & & & & \\
\hline$M$ & 5615.72 & 17802.21 & +0.01 & $76^{\circ}-47$ & & & & & & & \\
\hline$M$ & 5605.25 & 17835.46 & -0.03 & $103^{\circ}-107$ & & & & & & & \\
\hline$M$ & 5601.620 & 17847.04 & +0.12 & $97^{\circ}-60 ?$ & & & & & & & \\
\hline$M$ & 5599.665 & 17853.25 & -0.01 & $65^{\circ}-32$ & & & & & & & \\
\hline$M$ & 5593.615 & 17872.58 & +0.01 & $68^{\circ}-15$ & $M$ & 5 & $(0.145)$ & 1.503 & 1.077 & 0.793 & \\
\hline $\mathrm{C}$ & 5590.2 & 17883.5 & +0.1 & $50^{\circ}-68 ?$ & & & & & & & \\
\hline$c$ & 5588.87 & 17887.75 & 0.00 & $25^{\circ}-1$ & & & & & & & \\
\hline$M$ & 5583.759 & 17904.11 & -0.02 & $30^{\circ}-52$ & $S$ & & $(0)$ & 1.46 & & & \\
\hline$M$ & 5573.355 & 17937.53 & -0.05 & $26^{\circ}-80$ & & & & & & & \\
\hline$M$ & 5568.019 & 17954.72 & 0.00 & $99^{\circ}-141$ & $S$ & & $(0)$ & 1.47 & & & \\
\hline$M, K$ & 5565.015 & 17964.43 & 0.00 & $133^{\circ}-140$ & & & & & & & \\
\hline & & & +0.14 & $89^{\circ}-97 ?$ & & & & & & & \\
\hline$M$ & 5564.204 & 17967.03 & -0.03 & $98^{\circ}-140$ & $M$ & 5 & $(0.071)$ & 1.739 & 0.865 & 1.060 & \\
\hline$M$ & 5556.17 & 17993.01 & -0.06 & $139^{\circ}-91$ & & & & & & & \\
\hline$M$ & 5539.901 & 18045.86 & -0.05 & $53^{\circ}-21$ & $M$ & 4 & & 0.581 & $(0.601)$ & 0.614 & \\
\hline & & & & & $L$ & & $(0)$ & 0.57 & & & \\
\hline
\end{tabular}


Table 6 (cont inued)

\begin{tabular}{|c|c|c|c|c|c|c|c|c|c|c|c|}
\hline \multirow{3}{*}{ Reference } & \multirow{3}{*}{$\begin{array}{c}\text { Wave } \\
\text { Length } \\
\lambda(\AA)\end{array}$} & \multicolumn{2}{|c|}{ Frequency } & \multirow{3}{*}{ Transition } & \multirow{3}{*}{ Reference } & \multirow{2}{*}{\multicolumn{5}{|c|}{ Observed Zeeman Pattern }} & \multirow{3}{*}{$\begin{array}{c}\sigma_{230}-\sigma_{232} \\
\left(\mathrm{~cm}^{-1}\right)\end{array}$} \\
\hline & & \multirow{2}{*}{$\begin{array}{c}\sigma_{\mathrm{vac}} \\
\left(\mathrm{cm}^{-1}\right)\end{array}$} & \multirow{2}{*}{$\begin{array}{c}\Delta \sigma_{\mathrm{vac}} \\
\left(\mathrm{cm}^{-1}\right)\end{array}$} & & & & & & & & \\
\hline & & & & & & Type & $p$ & $n$ & $g_{1}$ & $s_{2}$ & \\
\hline$M$ & 5537.127 & 18054.89 & -0.01 & $95^{\circ}-97$ & $S$ & & $(0.41)$ & 1.01 & & & \\
\hline$M$ & 5528.003 & 18084.69 & -0.03 & $105^{\circ} \cdot 110$ & & & & & & & \\
\hline$M$ & 5524.212 & 18097.10 & -0.03 & $154^{\circ} \cdot 167$ & 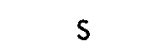 & & $(0)$ & 0.99 & & & \\
\hline \multirow[t]{2}{*}{$M$} & 5518.988 & 18114.23 & +0.02 & $21^{\circ}-26$ & & & & & & & \\
\hline & & & +0.09 & $110^{\circ}-81$ & & & & & & & \\
\hline \multirow[t]{2}{*}{ M } & 5510.678 & 18141.55 & +0.03 & $58^{\circ} \cdot 65$ & & & & & & & \\
\hline & & & -0.01 & $136^{\circ}-142$ & $\mathrm{~S}$ & & $(0.60)$ & & & & \\
\hline$M, K$ & 5507.985 & 18150.43 & -0.09 & $54^{\circ}-59$ & & & & & & & \\
\hline M & 5501.938 & 18170.38 & +0.08 & $26^{\circ} \cdot 1$ & $\mathrm{~S}$ & & $(0.53)$ & 0.59 & & & \\
\hline$M$ & 5495.805 & 18190.64 & +0.03 & $56^{\circ}-101$ & & & & & & & \\
\hline$c$ & 5489.10 & 18212.88 & +0.11 & $108^{\circ}-152 ?$ & & & & & & & \\
\hline$M$ & 5488.622 & 18214.45 & -0.02 & $21^{\circ} \cdot 13$ & & & & & & & \\
\hline$M$ & 5481.48 & 18238.18 & +0.09 & $109^{\circ}-78$ & s & & $(0)$ & 0.97 & & & \\
\hline$M$ & 5480.888 & 18240.15 & +0.03 & $72^{\circ}-93$ & $\mathrm{~s}$ & & (0) & 1.48 & & & \\
\hline$c$ & 5479.06 & 18246.25 & -0.04 & $58^{\circ} \cdot 104$ & & & & & & & \\
\hline \multirow[t]{2}{*}{$c$} & 5477.7 & 18250.8 & 0.0 & $104^{\circ}-150 ?$ & & & & & & & \\
\hline & & & 0.0 & $107^{\circ}-115 ?$ & & & & & & & \\
\hline$K$ & 5477.07 & 18252.88 & +0.03 & $137^{\circ}-168$ & $S$ & & $(0)$ & 0.85 & & & \\
\hline \multirow[t]{2}{*}{$M$} & 5474.862 & 18260.23 & +0.03 & $63^{\circ}-14$ & $M$ & & $(0.080)$ & & 0.746 & $(0.586)$ & \\
\hline & & & & & $S$ & & $(0)$ & 0.90 & & & \\
\hline K & 5468.79 & 18280.51 & -0.15 & $60^{\circ}-67 ?$ & & & & & & & \\
\hline$M$ & 5462.615 & 18301.18 & +0.02 & $135^{\circ}-100$ & $M$ & & $(0.114)$ & & $(0.791)$ & 1.022 & \\
\hline \multirow[t]{2}{*}{$M$} & 5461.738 & 18304.10 & +0.05 & $20^{\circ}-5$ & & & & & & & \\
\hline & & & +0.01 & $134^{\circ}-141$ & $S$ & & $(0)$ & 1.20 & & & \\
\hline \multirow[t]{2}{*}{$M$} & 5449.478 & 18345.30 & +0.01 & $135^{\circ}-166$ & $M$ & 5 & & 0.941 & $(0.791)$ & 0.818 & \\
\hline & & & +0.02 & $61^{\circ}-26$ & & & & & & & \\
\hline$V$ & 5447.33 & 18352.52 & +0.01 & $156^{\circ}-153$ & & & & & & & \\
\hline $\mathrm{K}$ & 5446.11 & 18356.64 & +0.06 & $159^{\circ}-181$ & & & & & & & \\
\hline$M$ & & & -0.10 & $133^{\circ}-99 ?$ & $\mathbf{L}$ & & (0) & 1.31 & & & \\
\hline
\end{tabular}


Table 6 (cont inued)

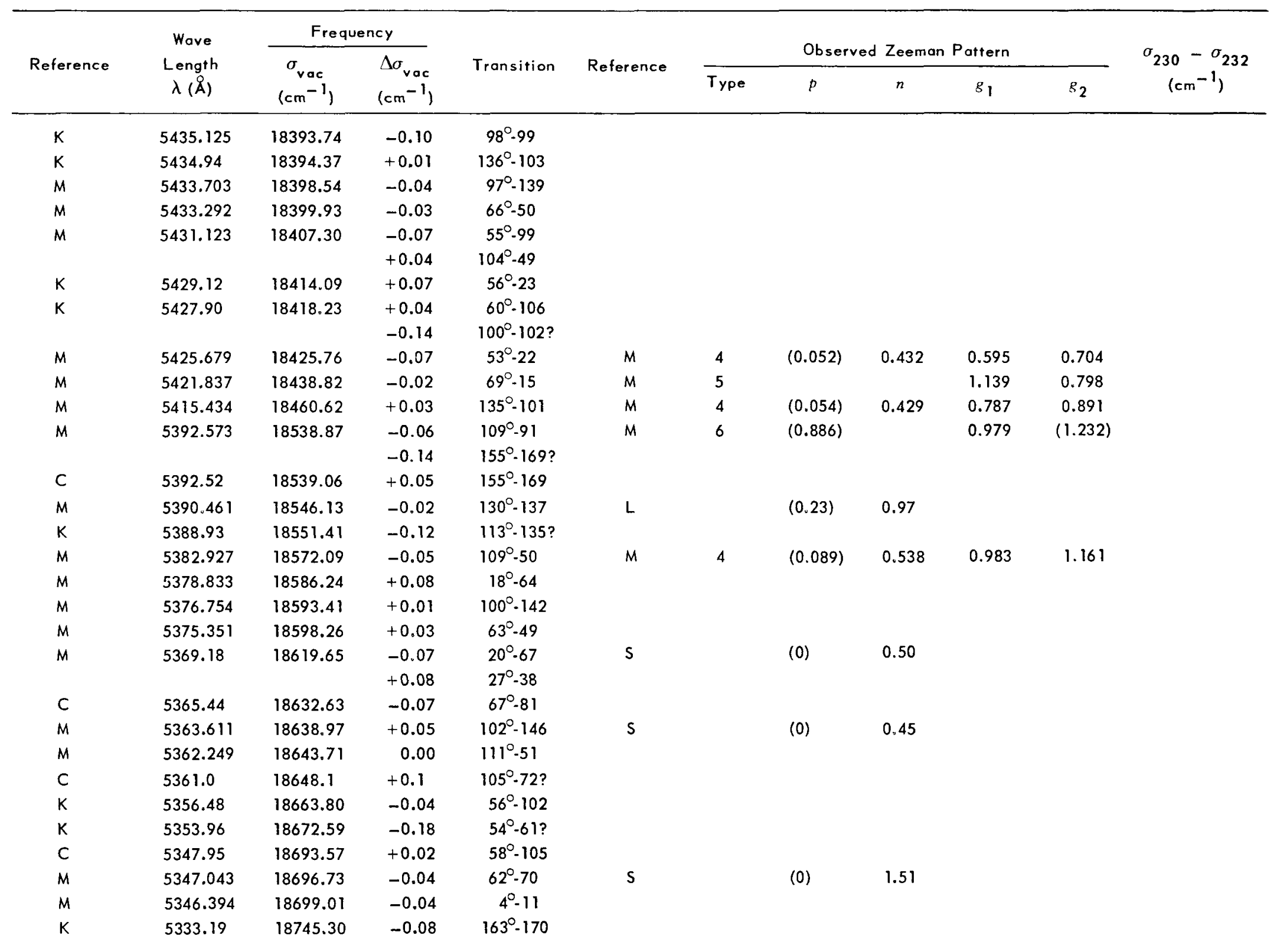


Table 6 (continued)

\begin{tabular}{|c|c|c|c|c|c|c|c|c|c|c|c|}
\hline \multirow{3}{*}{ Reference } & \multirow{3}{*}{$\begin{array}{c}\text { Wave } \\
\text { Length } \\
\lambda(\AA)\end{array}$} & \multicolumn{2}{|c|}{ Frequency } & \multirow{3}{*}{ Transition } & \multirow{3}{*}{ Reference } & \multirow{2}{*}{\multicolumn{5}{|c|}{ Observed Zeeman Pattern }} & \multirow{3}{*}{$\begin{array}{c}\sigma_{230}-\sigma_{232} \\
\left(\mathrm{~cm}^{-1}\right)\end{array}$} \\
\hline & & \multirow{2}{*}{$\begin{array}{c}\sigma_{v o c} \\
\left(\mathrm{~cm}^{-1}\right)\end{array}$} & \multirow{2}{*}{$\begin{array}{l}\Delta \sigma_{\mathrm{vac}} \\
\left(\mathrm{cm}^{-1}\right)\end{array}$} & & & & & & & & \\
\hline & & & & & & Type & $p$ & $n$ & $g_{1}$ & $g_{2}$ & \\
\hline C & 5330.04 & 18756.38 & 0.00 & $140^{\circ}-155$ & & & & & & & \\
\hline C & 5329.74 & 18757.43 & +0.07 & $97^{\circ}-98$ & & & & & & & \\
\hline \multirow[t]{2}{*}{ K } & 5329.43 & 18758.52 & +0.01 & $163^{\circ} \cdot 183$ & & & & & & & \\
\hline & & & +0.12 & $99^{\circ}-100 ?$ & & & & & & & \\
\hline K & 5325.157 & 18773.58 & -0.04 & $98^{\circ} .62$ & $L$ & & (0) & 0.91 & & & \\
\hline$M$ & 5318.423 & 18797.35 & +0.03 & $31^{\circ} \cdot 51$ & $\mathrm{~S}$ & & $(0)$ & 0.97 & & & \\
\hline \multirow[t]{2}{*}{$M$} & 5310.260 & 18826.23 & -0.05 & $64^{\circ} \cdot 14$ & & & & & & & \\
\hline & & & -0.05 & $101^{\circ}-104$ & & & & & & & \\
\hline M & 5305.580 & 18842.84 & -0.06 & $71^{\circ}-53$ & 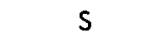 & & $(0.24)$ & 1.00 & & & \\
\hline$M$ & 5304.625 & 18846.23 & +0.03 & $100^{\circ}-103$ & $s$ & & $(0)$ & 0.92 & & & \\
\hline$M$ & 5303.046 & 18551.86 & +0.03 & $17^{\circ}-63$ & $S$ & & $(0)$ & 0.71 & & & \\
\hline$M$ & 5301.406 & 18857.67 & -0.06 & $2^{\circ}-23$ & $M$ & 5 & $(0.175)$ & 1.051 & 0.526 & 0.876 & \\
\hline$M$ & 5295.618 & 18878.29 & -0.05 & $94^{\circ}-97$ & & & & & & & \\
\hline V & 5291.86 & 18891.68 & +0.11 & $154^{\circ}-145 ?$ & & & & & & & \\
\hline$M$ & 5289.895 & 18898.72 & +0.05 & $23^{\circ} \cdot 50$ & $M$ & 5 & & & 1.020 & 1.162 & \\
\hline$M$ & 5284.549 & 18917.83 & 0.00 & $99^{\circ} \cdot 101$ & $S$ & & $(0.78)$ & & & & \\
\hline$M$ & 5280.090 & 18933.80 & -0.02 & $135^{\circ}-102$ & & & & & & & \\
\hline \multirow[t]{2}{*}{$M$} & 5277.502 & 18943.10 & 0.00 & $132^{\circ}-139$ & $M$ & 6 & $(0.446)$ & & $(0.931)$ & 1.030 & \\
\hline & & & & & $L$ & & $(0.41)$ & 0.97 & & & \\
\hline$M$ & 5276.407 & 18947.02 & 0.00 & $30^{\circ}-16$ & & & & & & & \\
\hline$M$ & 5272.646 & 18960.53 & -0.03 & $154^{\circ}-168$ & $S$ & & $(0.21)$ & 0.98 & & & \\
\hline K & 5268.52 & 18975.40 & +0.01 & $78^{\circ} \cdot 19$ & & & & & & & \\
\hline \multirow[t]{2}{*}{$M$} & 5264.96 & 18988.22 & -0.14 & $73^{\circ}-132$ & & & & & & & \\
\hline & & & -0.15 & $105^{\circ}-114$ & & & & & & & \\
\hline$C$ & 5261.45 & 19000.89 & +0.07 & $57^{\circ}-65$ & & & & & & & \\
\hline$M$ & 5255.96 & 19020.72 & +0.13 & $141^{\circ}-121 ?$ & & & & & & & \\
\hline$M$ & 5255.52 & 19022.33 & +0.05 & $4^{\circ} \cdot 1$ & & & & & & & \\
\hline$M$ & 5253.455 & 19029.81 & +0.02 & $18^{\circ}-65$ & & & & & & & \\
\hline $\mathrm{C}$ & 5241.84 & 19071.97 & +0.09 & $19^{\circ} \cdot 4$ & & & & & & & \\
\hline$M$ & 5240.194 & 19077.95 & -0.01 & $93^{\circ}-137$ & $S$ & & $(0)$ & 1.63 & & & \\
\hline
\end{tabular}


Table 6 (cont inued)

\begin{tabular}{|c|c|c|c|c|c|c|c|c|c|c|c|}
\hline \multirow{3}{*}{ Reference } & \multirow{3}{*}{$\begin{array}{c}\text { Wave } \\
\text { Length } \\
\lambda(\AA)\end{array}$} & \multicolumn{2}{|c|}{ Frequency } & \multirow{3}{*}{ Transition } & \multirow{3}{*}{ Reference } & \multirow{2}{*}{\multicolumn{5}{|c|}{ Observed Zeeman Pattern }} & \multirow{3}{*}{$\begin{array}{c}\sigma_{230}-\sigma_{232} \\
\left(\mathrm{~cm}^{-1}\right)\end{array}$} \\
\hline & & \multirow{2}{*}{$\begin{array}{c}\sigma_{\mathrm{vac}} \\
\left(\mathrm{cm}^{-1}\right)\end{array}$} & \multirow{2}{*}{$\begin{array}{c}\Delta \sigma_{\mathrm{vac}} \\
\left(\mathrm{cm}^{-1}\right)\end{array}$} & & & & & & & & \\
\hline & & & & & & Type & $p$ & $n$ & $g_{1}$ & $g_{2}$ & \\
\hline$K$ & 5236.78 & 19090.41 & -0.16 & $101^{\circ}-66 ?$ & & & & & & & \\
\hline$M$ & 5233.226 & 19103.37 & -0.01 & $96^{\circ} .59$ & $M$ & 5 & $(0.095)$ & 1.642 & 1.174 & 0.988 & \\
\hline$M$ & 5232.016 & 19107.77 & 0.00 & $134^{\circ}-100$ & & & & & & & \\
\hline$M$ & 5231.159 & 19110.90 & +0.06 & $150^{\circ}-97$ & & & & & & & \\
\hline C & 5220.95 & 19148.29 & +0.14 & $94^{\circ}-138 ?$ & & & & & & & \\
\hline$M$ & 5218.528 & 19157.17 & +0.04 & $52^{\circ}-56$ & & & & & & & \\
\hline$M$ & 5216.590 & 19164.27 & -0.05 & $64^{\circ}-49$ & $S$ & & $(0.29)$ & 1.04 & & & \\
\hline$M$ & 5214.15 & 19173.25 & +0.05 & $164^{\circ} \cdot 185$ & $s$ & & $(0.35)$ & 1.17 & & & \\
\hline$M$ & 5206.659 & 19200.83 & +0.02 & $54^{\circ}-98$ & $s$ & & $(0)$ & 1.18 & & & \\
\hline \multirow[t]{2}{*}{$M$} & 5205.20 & 19206.22 & +0.05 & $103^{\circ}-150$ & & & & & & & \\
\hline & & & +0.11 & $142^{\circ}-158 ?$ & & & & & & & \\
\hline$M$ & 5198.832 & 19229.73 & +0.10 & $113^{\circ}-94$ & $L$ & & (0) & 1.15 & & & \\
\hline$M$ & 5195.814 & 19240.90 & +0.01 & $108^{\circ}-78$ & & & & & & & \\
\hline$M$ & 5194.22 & 19246.83 & +0.07 & $63^{\circ}-74$ & $M$ & 5 & & $1.042^{\star}$ & & & \\
\hline$M$ & 5193.820 & 19248.31 & -0.01 & $62^{\circ}-13$ & $M$ & & & & 0.930 & $(0.639)$ & \\
\hline$M$ & 5190.870 & 19259.24 & +0.03 & $24^{\circ}-50$ & $M$ & 5 & & & 1.075 & 1.166 & \\
\hline$M$ & 5188.371 & 19268.51 & -0.03 & $98^{\circ}-63$ & & & & & & & \\
\hline$M$ & 5184.726 & 19282.07 & 0.00 & $55^{\circ}-63$ & & & & & & & \\
\hline $\mathrm{K}$ & 5184.011 & 19284.73 & -0.02 & $133^{\circ}-141$ & $L$ & & $(0)$ & 1.29 & & & \\
\hline$K$ & 5179.41 & 19301.86 & -0.02 & $132^{\circ}-98$ & & & & & & & \\
\hline$K$ & 5176.75 & 19311.79 & -0.04 & $153^{\circ}-167$ & & & & & & & \\
\hline$M$ & 5174.198 & 19321.27 & -0.05 & $22^{\circ}-29$ & & & & & & & \\
\hline$K$ & 5172.95 & 19325.96 & -0.13 & $151^{\circ}-139 ?$ & & & & & & & \\
\hline $\mathrm{K}$ & 5170.23 & 19336.13 & -0.03 & $97^{\circ}-140$ & K & & $(0)$ & 1.17 & & & \\
\hline \multirow[t]{2}{*}{$M$} & 5166.902 & 19348.57 & +0.01 & $139^{\circ}-117$ & & & & & & & \\
\hline & & & -0.02 & $60^{\circ}-107$ & & & & & & & \\
\hline$M$ & 5163.446 & 19361.53 & -0.12 & $135^{\circ}-103$ & & & & & & & \\
\hline$M$ & 5160.695 & 19371.84 & -0.01 & $130^{\circ}-96$ & $\mathrm{~L}$ & & $(0)$ & 1.22 & & & \\
\hline$M$ & 5151.620 & 19405.98 & +0.18 & $7^{\circ}-18 ?$ & & & & & & & \\
\hline$K$ & 5148.210 & 19418.83 & -0.03 & $136^{\circ}-167$ & $L$ & & (0) & 1.03 & & & \\
\hline$M$ & 5145.028 & 19430.83 & +0.06 & $52^{\circ}-20$ & & & & & & & \\
\hline
\end{tabular}

*These data are inconsistent with the classifications listed. 
Table 6 (continued)

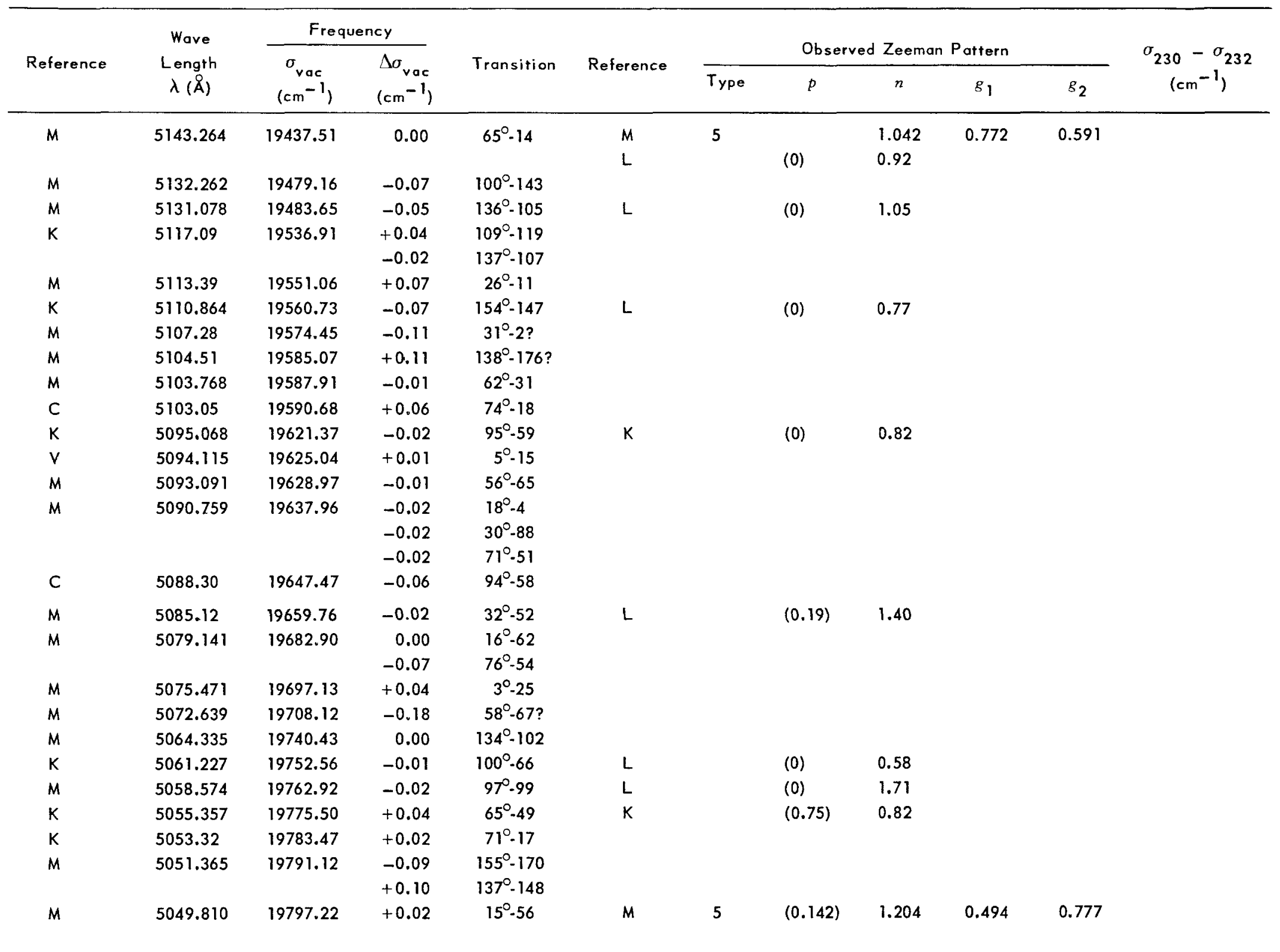


Table 6 (cont inued)

\begin{tabular}{|c|c|c|c|c|c|c|c|c|c|c|c|}
\hline \multirow{3}{*}{ Reference } & \multirow{3}{*}{$\begin{array}{c}\text { Wave } \\
\text { Length } \\
\lambda(\AA)\end{array}$} & \multicolumn{2}{|c|}{ Frequency } & \multirow{3}{*}{ Transition } & \multirow{3}{*}{ Reference } & \multirow{2}{*}{\multicolumn{5}{|c|}{ Observed Zeeman Pattern }} & \multirow{3}{*}{$\begin{array}{c}\sigma_{230}-\sigma_{232} \\
\left(\mathrm{~cm}^{-1}\right)\end{array}$} \\
\hline & & $\sigma_{\text {vor }}$ & $\Delta \sigma_{\text {var }}$ & & & & & & & & \\
\hline & & $\left(\mathrm{cm}^{-1}\right)$ & $\left(\mathrm{cm}^{-1}\right)$ & & & Type & $p$ & $n$ & $g_{1}$ & $g_{2}$ & \\
\hline $\mathrm{K}$ & 5047.429 & 19806.55 & +0.04 & $115^{\circ}-55$ & $\mathrm{k}$ & & (0) & 0.78 & & & \\
\hline M & 5039.231 & 19838.77 & -0.09 & $66^{\circ} \cdot 80$ & & & & & & & \\
\hline \multirow[t]{2}{*}{ M } & 5029.631 & 19876.65 & +0.04 & $18^{\circ}-25$ & & & & & & & \\
\hline & & & -0.08 & $140^{\circ} \cdot 120$ & & & & & & & \\
\hline M & 5028.609 & 19880.69 & +0.01 & $132^{\circ} \cdot 140$ & M & 6 & $(0.476)$ & 0.985 & 0.932 & 1.037 & 0.27 \\
\hline M & 5023.052 & 19902.67 & +0.10 & $102^{\circ}-107$ & & & & & & & \\
\hline M & 5020.541 & 19912.62 & +0.05 & $99^{\circ}-64$ & & & & & & & \\
\hline c & 5019.80 & 19915.57 & +0.11 & $134^{\circ}-142 ?$ & & & & & & & \\
\hline M & 5019.326 & 19917.46 & +0.07 & $2^{\circ}-24$ & & & & & & & \\
\hline M & 5017.247 & 19925.71 & +0.03 & $52^{\circ}-97$ & M & 4 & & 0.986 & $(1.061)$ & 1.040 & \\
\hline M & 5014.757 & 19935.58 & +0.04 & $100^{\circ} \cdot 105$ & & & & & & & \\
\hline M & 5008.194 & 19961.71 & +0.03 & $61^{\circ}-70$ & L & & (0) & 0.94 & & & \\
\hline M & 4999.938 & 19994.69 & +0.01 & $135^{\circ}-143$ & & & & & & & \\
\hline M & 4999.109 & 19997.98 & -0.06 & $56^{\circ}-66$ & & & & & & & \\
\hline c & 4997.83 & 20003.1 & 0.0 & $101^{\circ}-145$ & & & & & & & \\
\hline M & 4997.327 & 20005.12 & +0.07 & $137^{\circ}-108$ & & & & & & & \\
\hline $\mathrm{k}$ & 4993.92 & 20018.8 & +0.1 & $25^{\circ}-50 ?$ & $\mathrm{~K}$ & & (0) & 1. $10^{*}$ & & & \\
\hline M & 4990.031 & 20034.36 & -0.02 & $72^{\circ}-52$ & & & & & & & \\
\hline K & 4987.160 & 20045.92 & -0.02 & $163^{\circ}-173$ & $\mathrm{k}$ & & (0) & 1.19 & & & \\
\hline \multirow[t]{2}{*}{ K } & 4980.954 & 20070.89 & +0.05 & $15^{\circ}-20$ & K & & (0) & 0.58 & & & \\
\hline & & & 0.00 & $106^{\circ}-78$ & & & & & & & \\
\hline M & 4976.597 & 20088.45 & +0.02 & $133^{\circ}-100$ & & & & & & & \\
\hline K & 4975.94 & 20091.1 & +0.1 & $98^{\circ}-100$ & $\mathrm{k}$ & & $(0.58)$ & 0.81 & & & \\
\hline M & 4973.394 & 20101.40 & 0.00 & $3^{0}-5$ & & & & & & & \\
\hline c & 4973.07 & 20102.7 & -0.1 & $63^{\circ}-33$ & & & & & & & \\
\hline \multirow[t]{2}{*}{ M } & 4972.609 & 20104.57 & -0.02 & $55^{\circ} \cdot 100$ & & & & & & & \\
\hline & & & -0.16 & $77^{\circ}-54 ?$ & & & & & & & \\
\hline M & 4972.179 & 20106.30 & +0.03 & $153^{\circ}-145$ & & & & & & & \\
\hline $\mathrm{K}$ & 4970.032 & 20114.98 & -0.03 & $32^{\circ}-51$ & K & & (0) & 0.84 & & & \\
\hline M & 4968.755 & 20120.16 & +0.02 & $63^{\circ} .13$ & & & & & & & \\
\hline c & 4963.78 & 20140.3 & 0.0 & $158^{\circ}-190$ & & & & & & & \\
\hline
\end{tabular}

*These data are inconsistent with the classifications listed. 
Table 6 (continued)

\begin{tabular}{|c|c|c|c|c|c|c|c|c|c|c|c|}
\hline \multirow{3}{*}{ Reference } & \multirow{3}{*}{$\begin{array}{c}\text { Wave } \\
\text { Length } \\
\lambda(\AA)\end{array}$} & \multicolumn{2}{|c|}{ Frequency } & \multirow{3}{*}{ Transition } & \multirow{3}{*}{ Reference } & \multirow{2}{*}{\multicolumn{5}{|c|}{ Observed Zeeman Pattern }} & \multirow{3}{*}{$\begin{array}{c}\sigma_{230}-\sigma_{232} \\
\left(\mathrm{~cm}^{-1}\right)\end{array}$} \\
\hline & & & $\Delta \sigma$ & & & & & & & & \\
\hline & & $\left(\begin{array}{c}v a c \\
\left(\mathrm{~cm}^{-1}\right)\end{array}\right.$ & $\left(\mathrm{cm}^{-1}\right)$ & & & Type & $p$ & $n$ & $g_{1}$ & $g_{2}$ & \\
\hline$M$ & 4963.192 & 20142.70 & -0.02 & $97^{\circ} .62$ & & & & & & & \\
\hline$M$ & 4962.963 & 20143.63 & -0.01 & $95^{\circ}-61$ & & & & & & & \\
\hline$M$ & 4960.494 & 20153.65 & -0.02 & $96^{\circ}-98$ & & & & & & & \\
\hline$M$ & 4958.725 & 20160.85 & +0.06 & $108^{\circ}-49$ & & & & & & & \\
\hline $\mathrm{K}$ & 4956.89 & 20168.3 & +0.1 & $134^{\circ}-103$ & & & & & & & \\
\hline$k$ & 4954.649 & 20177.45 & +0.06 & $152^{\circ} \cdot 167$ & K & & $(0.39)$ & 1.03 & & & \\
\hline \multirow[t]{2}{*}{$\mathrm{K}$} & 4954.569 & 20177.77 & -0.05 & $16^{\circ}-63$ & $M$ & 5 & & 1.132 & 0.877 & 0.977 & \\
\hline & & & & & $\mathrm{L}$ & & $(0)$ & 1.04 & & & \\
\hline $\mathrm{C}$ & 4952.68 & 20185.5 & +0.1 & $156^{\circ}-157$ & & & & & & & \\
\hline$M$ & 4951.391 & 20190.71 & -0.04 & $65^{\circ} \cdot 117$ & & & & & & & \\
\hline K & 4947.567 & 20206.33 & -0.06 & $54^{\circ}-99$ & K & & $(0)$ & 1.49 & & & \\
\hline$M$ & 4946.661 & 20210.01 & -0.03 & $62^{\circ}-115$ & & & & & & & \\
\hline$M$ & 4945.850 & 20213.33 & +0.03 & $136^{\circ}-145$ & & & & & & & \\
\hline$K$ & 4939.834 & 20237.96 & -0.12 & $66^{\circ}-121 ?$ & & & & & & & \\
\hline$K$ & 4936.760 & 20250.57 & +0.08 & $98^{\circ}-101$ & $K$ & & $(0.17)$ & 0.87 & & & \\
\hline$K$ & 4933.852 & 20262.50 & -0.01 & $52^{\circ}-57$ & $K$ & & $(0.22)$ & 0.95 & & & \\
\hline$M$ & 4933.541 & 20263.76 & +0.09 & $151^{\circ}-140$ & $K$ & & $(0)$ & $0.84^{\star}$ & & & \\
\hline $\mathrm{K}$ & 4933.51 & 20263.9 & -0.1 & $55^{\circ}-101 ?$ & $K$ & & (0) & 0.84 & & & \\
\hline$M$ & 4929.38 & 20280.9 & 0.0 & $18^{\circ}-5$ & & & & & & & \\
\hline c & 4929.06 & 20282.2 & -0.1 & $136^{\circ}-168$ & & & & & & & \\
\hline$M$ & 4927.587 & 20288.25 & -0.05 & $101^{\circ}-67$ & & & & & & & \\
\hline$M$ & 4925.789 & 20295.65 & +0.01 & $58^{\circ}-26$ & & & & & & & \\
\hline$M$ & 4924.431 & 20301.26 & +0.02 & $26^{\circ}-50$ & $\mathrm{~K}$ & & $(0)$ & 1.26 & & & \\
\hline $\mathrm{K}$ & 4922.940 & 20307.41 & -0.05 & $132^{\circ}-99$ & $K$ & & $(0.13)$ & 0 & & & \\
\hline $\mathrm{K}$ & 4921.608 & 20312.91 & +0.01 & $95^{\circ}-139$ & $\mathrm{~K}$ & & (0) & 1.30 & & & \\
\hline $\mathrm{K}$ & 4920.53 & 20317.4 & +0.1 & $67^{\circ} .91$ & & & & & & & \\
\hline$M$ & 4919.814 & 20320.32 & +0.06 & $93^{\circ}-56$ & $M$ & 4 & & 0.582 & $(0.718)$ & 0.772 & \\
\hline $\mathrm{K}$ & 4918.14 & 20327.2 & 0.0 & $60^{\circ}-109$ & & & & & & & \\
\hline$M$ & 4914.122 & 20343.85 & +0.01 & $20^{\circ}-69$ & & & & & & & \\
\hline$M$ & 4912.529 & 20350.45 & -0.05 & $67^{\circ}-50$ & $\mathrm{~K}$ & & $(0.57)$ & 1.30 & & & \\
\hline $\mathrm{K}$ & 4911.12 & 20356.3 & +0.1 & $99^{\circ}-65$ & $K$ & & $(0)$ & 1.47 & & & \\
\hline
\end{tabular}

*These data are inconsistent with the classifications listed. 


\begin{tabular}{|c|c|c|c|c|c|c|c|c|c|c|c|}
\hline \multirow{3}{*}{ Reference } & \multirow{3}{*}{$\begin{array}{c}\text { Wave } \\
\text { Length } \\
\lambda(\AA)\end{array}$} & \multicolumn{2}{|c|}{ Frequency } & \multirow{3}{*}{ Transition } & \multirow{3}{*}{ Reference } & \multirow{2}{*}{\multicolumn{5}{|c|}{ Observed Zeeman Pattern }} & \multirow{3}{*}{$\begin{array}{c}\sigma_{230}-\sigma_{232} \\
\left(\mathrm{~cm}^{-1}\right)\end{array}$} \\
\hline & & $\sigma_{\text {vac }}$ & $\Delta \sigma_{\text {vac }}$ & & & & & & & & \\
\hline & & $\left(\mathrm{cm}^{-1}\right)$ & $\left(\mathrm{cm}^{-1}\right)$ & & & Type & $p$ & $n$ & $g_{1}$ & $g_{2}$ & \\
\hline M & 4902.776 & 20390.92 & -0.11 & $65^{\circ}-118 ?$ & & & & & & & \\
\hline M & 4899.151 & 20406.00 & -0.02 & $61^{\circ}-112$ & & & & & & & \\
\hline M & 4898.812 & 20407.42 & +0.03 & $137^{\circ} \cdot 171$ & & & & & & & \\
\hline K & 4898.452 & 20408.93 & +0.06 & $155^{\circ} \cdot 150$ & $\mathrm{~K}$ & & (0) & 1.58 & & & \\
\hline \multirow[t]{2}{*}{ M } & 4895.663 & 20420.56 & 0.00 & $20^{\circ} \cdot 27$ & & & & & & & \\
\hline & & & -0.07 & $25^{\circ} \cdot 84$ & & & & & & & \\
\hline M & 4889.853 & 20444.80 & -0.03 & $94^{\circ} .59$ & & & & & & & \\
\hline $\mathrm{k}$ & 4888.41 & 20450.9 & -0.1 & $135^{\circ} \cdot 105 ?$ & & & & & & & \\
\hline M & 4886.01 & 20460.9 & -0.1 & $99^{\circ} \cdot 104$ & & & & & & & \\
\hline \multirow[t]{2}{*}{ K } & 4882.45 & 20475.8 & 0.0 & $56^{\circ}-25$ & K & & $(0.12)$ & 1.81 & & & \\
\hline & & & +0.1 & $106^{\circ}-79 ?$ & & & & & & & \\
\hline C & 4881.22 & 20481.0 & +0.1 & $107^{\circ}-118 ?$ & & & & & & & \\
\hline C & 4880.90 & 20482.3 & -0.1 & $104^{\circ}-116$ & & & & & & & \\
\hline M & 4880.226 & 20485.13 & +0.09 & $140^{\circ}-179$ & & & & & & & \\
\hline M & 4879.158 & 20489.62 & +0.01 & $72^{\circ} .51$ & & & & & & & \\
\hline K & 4876.985 & 20498.76 & +0.03 & $154^{\circ}-148$ & L & & (0) & 0.90 & & & \\
\hline c & 4872.04 & 20519.6 & +0.1 & $5^{\circ}-12$ & & & & & & & \\
\hline M & 4868.274 & 20535.43 & +0.03 & $163^{\circ}-174$ & & & & & & & \\
\hline$M$ & 4866.6 & 20542.5 & +0.1 & $109^{\circ}-82 ?$ & & & & & & & \\
\hline \multirow[t]{2}{*}{$M, K$} & 4863.174 & 20556.97 & -0.03 & $130^{\circ}-97$ & $L$ & & $(0)$ & 1.00 & & & \\
\hline & & & 0.00 & $158^{\circ}-133$ & $L$ & & (0) & 1.00 & & & \\
\hline M & 4860.410 & 20568.66 & +0.09 & $137^{\circ} \cdot 149$ & & & & & & & \\
\hline M & 4856.277 & 20586.17 & 0.00 & $54^{\circ}-62$ & & & & & & & \\
\hline M & 4850.45 & 20660.9 & -0.1 & $144^{\circ}-91 ?$ & $\mathrm{~L}$ & & (0) & 0.99 & & & 0.64 \\
\hline k & 4850.03 & 20612.7 & 0.0 & $101^{\circ} .68$ & & & & & & & \\
\hline$k$ & 4849.42 & 20615.3 & +0.1 & $112^{\circ} \cdot 134$ & & & & & & & \\
\hline M & 4849.028 & 20616.95 & -0.04 & $165^{\circ}-187$ & L & & (0) & 1.55 & & & \\
\hline C & 4845.97 & 20630.0 & 0.0 & $156^{\circ} \cdot 187$ & & & & & & & \\
\hline M & 4844.759 & 20635.10 & +0.03 & $72^{\circ} \cdot 17$ & & & & & & & \\
\hline \multirow[t]{2}{*}{ K } & 4844.548 & 20636.01 & +0.08 & $131^{\circ}-98$ & $\mathrm{~K}$ & & (0) & 1.01 & & & \\
\hline & & & +0.02 & $136^{\circ} \cdot 106$ & K & & (0) & 1.01 & & & \\
\hline c & 4844.20 & 20637.5 & 0.0 & $144^{\circ}-127$ & $\mathrm{~L}$ & & (0) & 1.02 & & & \\
\hline K & 4844.164 & 20637.65 & +0.01 & $97^{\circ}-63$ & K & & (0) & 1.02 & & & \\
\hline
\end{tabular}


Table 6 (continued)

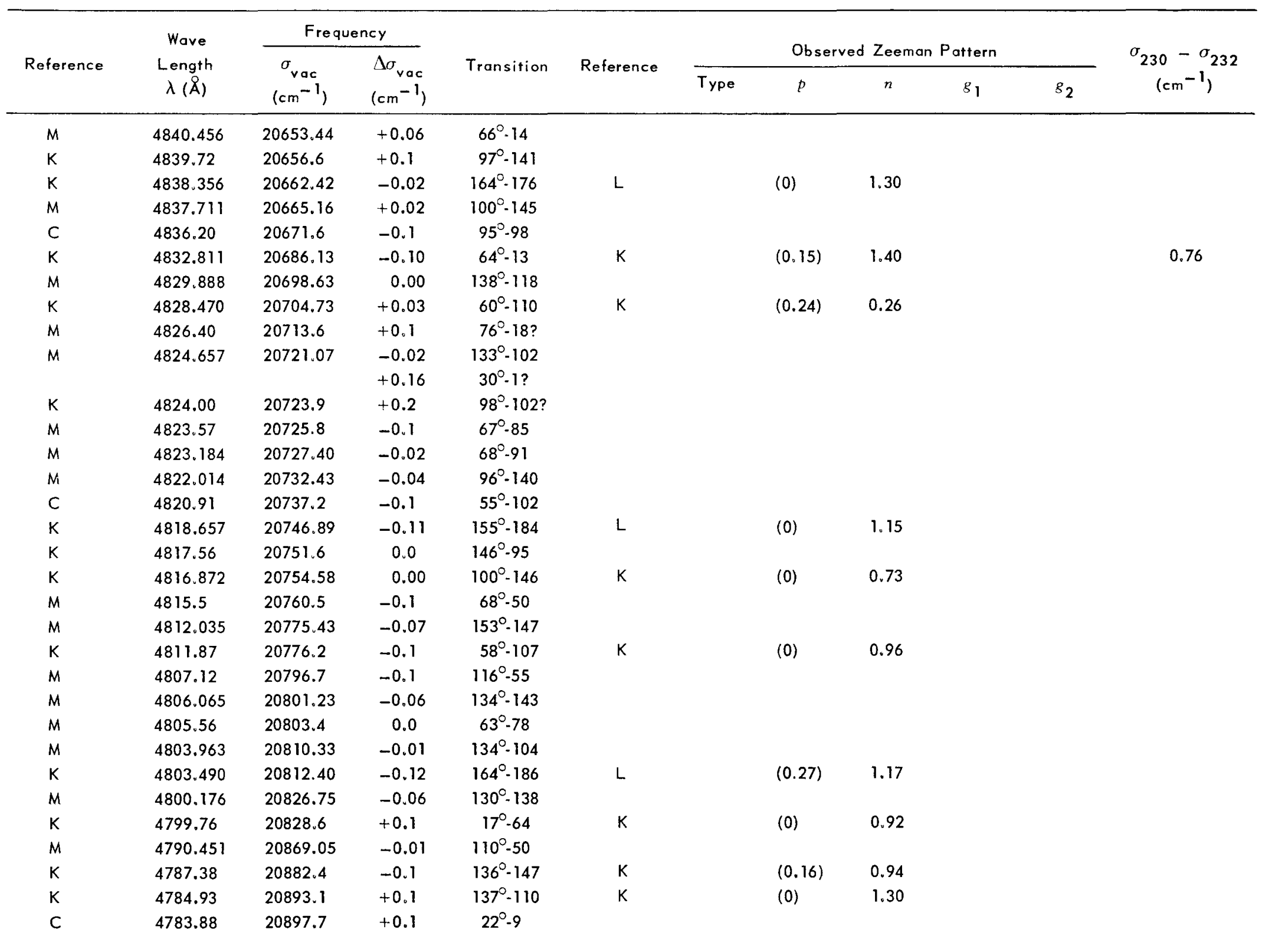


Table 6 (continued)

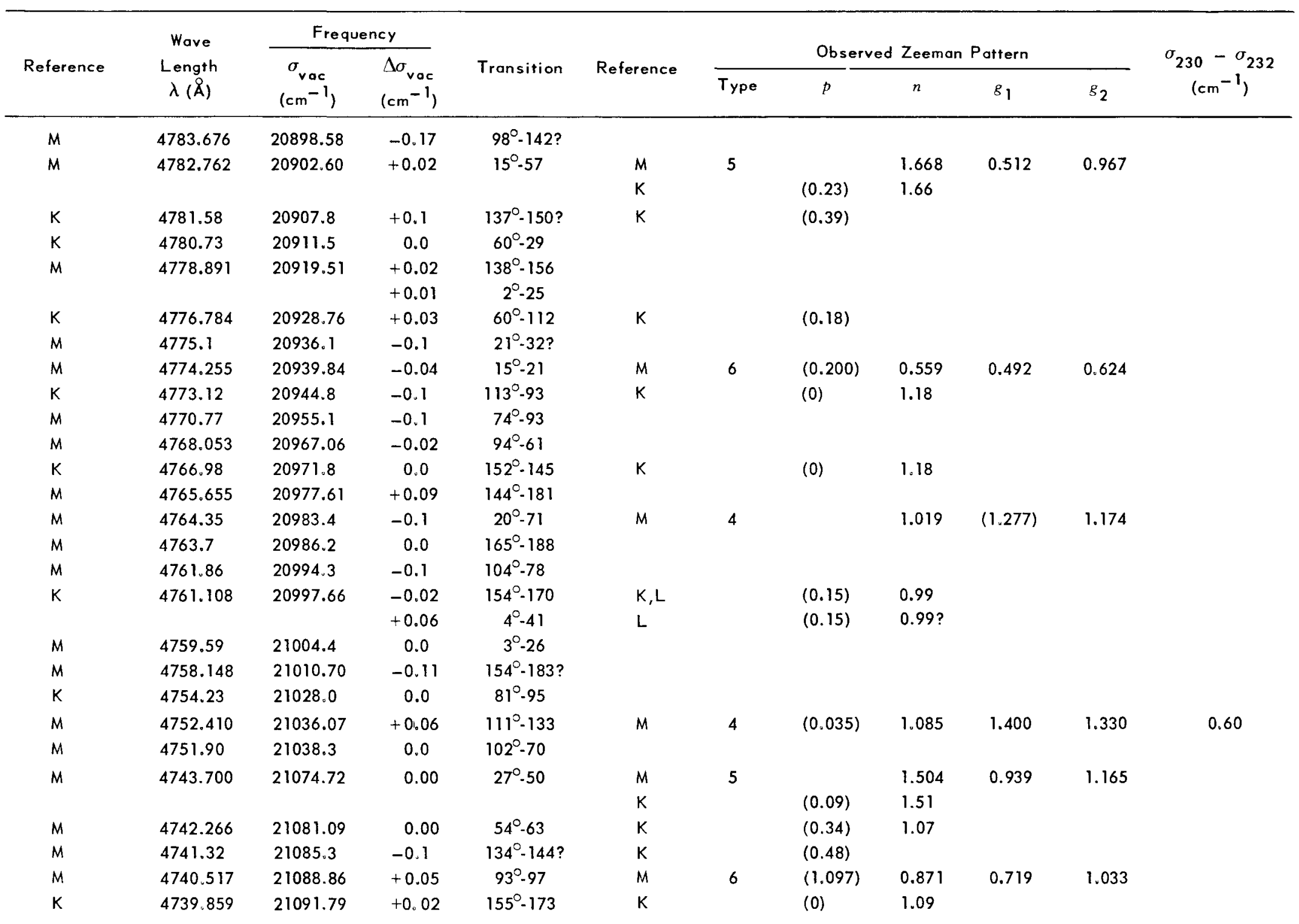


Table 6 (continued)

\begin{tabular}{|c|c|c|c|c|c|c|c|c|c|c|c|}
\hline \multirow{3}{*}{ Reference } & \multirow{3}{*}{$\begin{array}{c}\text { Wave } \\
\text { Length } \\
\lambda(\AA)\end{array}$} & \multicolumn{2}{|c|}{ Frequency } & \multirow{3}{*}{ Transition } & \multirow{3}{*}{ Reference } & \multirow{2}{*}{\multicolumn{5}{|c|}{ Observed Zeeman Pattern }} & \multirow{3}{*}{$\begin{array}{c}\sigma_{230}-\sigma_{232} \\
\left(\mathrm{~cm}^{-1}\right)\end{array}$} \\
\hline & & \multirow{2}{*}{$\begin{array}{c}\sigma_{\mathrm{vac}} \\
\left(\mathrm{cm}^{-1}\right)\end{array}$} & \multirow{2}{*}{$\begin{array}{l}\Delta \sigma_{\text {vac }} \\
\left(\mathrm{cm}^{-1}\right)\end{array}$} & & & & & & & & \\
\hline & & & & & & Type & $p$ & $n$ & $g_{1}$ & $g_{2}$ & \\
\hline$M$ & 4737.660 & 21101.57 & -0.04 & $19^{\circ} .7$ & & & & & & & \\
\hline$K$ & 4734.62 & 21115.1 & 0.0 & $154^{\circ} \cdot 171$ & & & & & & & \\
\hline$K$ & 4733.00 & 21122.4 & +0.2 & $163^{\circ}-175$ & $K$ & & $(0)$ & 0.99 & & & \\
\hline$M$ & 4732.680 & 21123.77 & +0.04 & $109^{\circ}-159$ & & & & & & & \\
\hline$M$ & 4729.883 & 21136.28 & -0.06 & $94^{\circ}-139$ & $M$ & 4 & & 0.684 & 1.125 & 1.026 & 0.62 \\
\hline$K$ & 4726.452 & 21151.62 & +0.07 & $98^{\circ}-103$ & $K$ & & $(0)$ & 0.82 & & & \\
\hline$M$ & 4726.339 & 21152.13 & +0.04 & $23^{\circ}-14$ & & & & & & & \\
\hline$M$ & 4725.95 & 21153.9 & 0.0 & $38^{\circ}-19$ & & & & & & & \\
\hline K & 4724.771 & 21159.15 & -0.10 & $96^{\circ} .99$ & $L$ & & $(0.14)$ & 1.19 & & & \\
\hline$K$ & 4723.775 & 21163.59 & +0.02 & $109^{\circ}-49$ & $K$ & & $(0)$ & 0.82 & & & \\
\hline & & & & & $M$ & 4 & $(0.044)$ & 0.801 & 1.018 & 1.106 & 0.66 \\
\hline$M$ & 4723.452 & $2\} 165.04$ & +0.06 & $159^{\circ} \cdot 133$ & $L$ & & $(0)$ & 0.60 & & & \\
\hline & & & -0.02 & $55^{\circ}-103$ & $L$ & & $(0)$ & 0.60 & & & \\
\hline$M$ & 4719.997 & 21180.55 & -0.04 & $135^{\circ}-145$ & $M$ & 6 & $(1.178)$ & 0.923 & 0.793 & 1.055 & \\
\hline$M$ & 4719.245 & 21183.92 & +0.01 & $18^{\circ}-26$ & & & & & & & \\
\hline$M$ & 4718.627 & 21186.69 & -0.02 & $164^{\circ}-191$ & $K$ & & $(0)$ & 1.19 & & & \\
\hline$M$ & 4716.002 & 21198.51 & +0.12 & $102^{\circ} \cdot 71 ?$ & & & & & & & \\
\hline$M$ & 4715.436 & 21201.03 & +0.03 & $132^{\circ}-141$ & $M$ & 6 & & 1.075 & 0.928 & 1.222 & \\
\hline & & & & & $\mathrm{K}$ & & $(1.28)$ & 1.06 & & & \\
\hline$K$ & 4713.82 & 21208.3 & +0.1 & $39^{\circ} .19$ & & & & & & & \\
\hline & & & +0.1 & $63^{\circ}-79$ & & & & & & & \\
\hline$M$ & 4712.395 & 21214.69 & -0.04 & $131^{\circ}-140$ & $K$ & & $(0.15)$ & 1.07 & & & \\
\hline$M$ & 4708.076 & 21234.18 & +0.12 & $1^{\circ}-23$ & $M$ & 5 & $(0.310)$ & 1.184 & 0.253 & 0.874 & \\
\hline$C$ & 4706.26 & 21242.4 & 0.0 & $103^{\circ}-76$ & & & & & & & \\
\hline$M$ & 4706.219 & 21242.55 & +0.13 & $71^{\circ}-15$ & $M$ & 5 & $(0.060)$ & 1.107 & 0.924 & 0.803 & \\
\hline $\mathrm{K}$ & 4705.622 & 21245.25 & +0.02 & $98^{\circ}-64$ & & & & & & & \\
\hline$K$ & 4704.65 & 21249.6 & +0.1 & $135^{\circ}-168$ & & & & & & & \\
\hline$K$ & 4704.49 & 21250.4 & 0.0 & $64^{\circ}-37$ & & & & & & & \\
\hline & & & -0.1 & $95^{\circ}-140$ & & & & & & & \\
\hline$M$ & 4702.320 & 21260.17 & +0.14 & $33^{\circ}-17 ?$ & $\mathrm{~K}$ & & (0) & 0.94 & & & \\
\hline & & & -0.06 & $73^{\circ} .92$ & & & & & & & \\
\hline$M$ & 4700.150 & 21269.99 & -0.04 & $135^{\circ} \cdot 146$ & $M$ & 6 & $(0.113)$ & 0.808 & 0.795 & 0.820 & \\
\hline$k$ & 4699.65 & 21272.2 & +0.1 & $17^{\circ} \cdot 65$ & & & & & & & \\
\hline
\end{tabular}


Table 6 (continued)

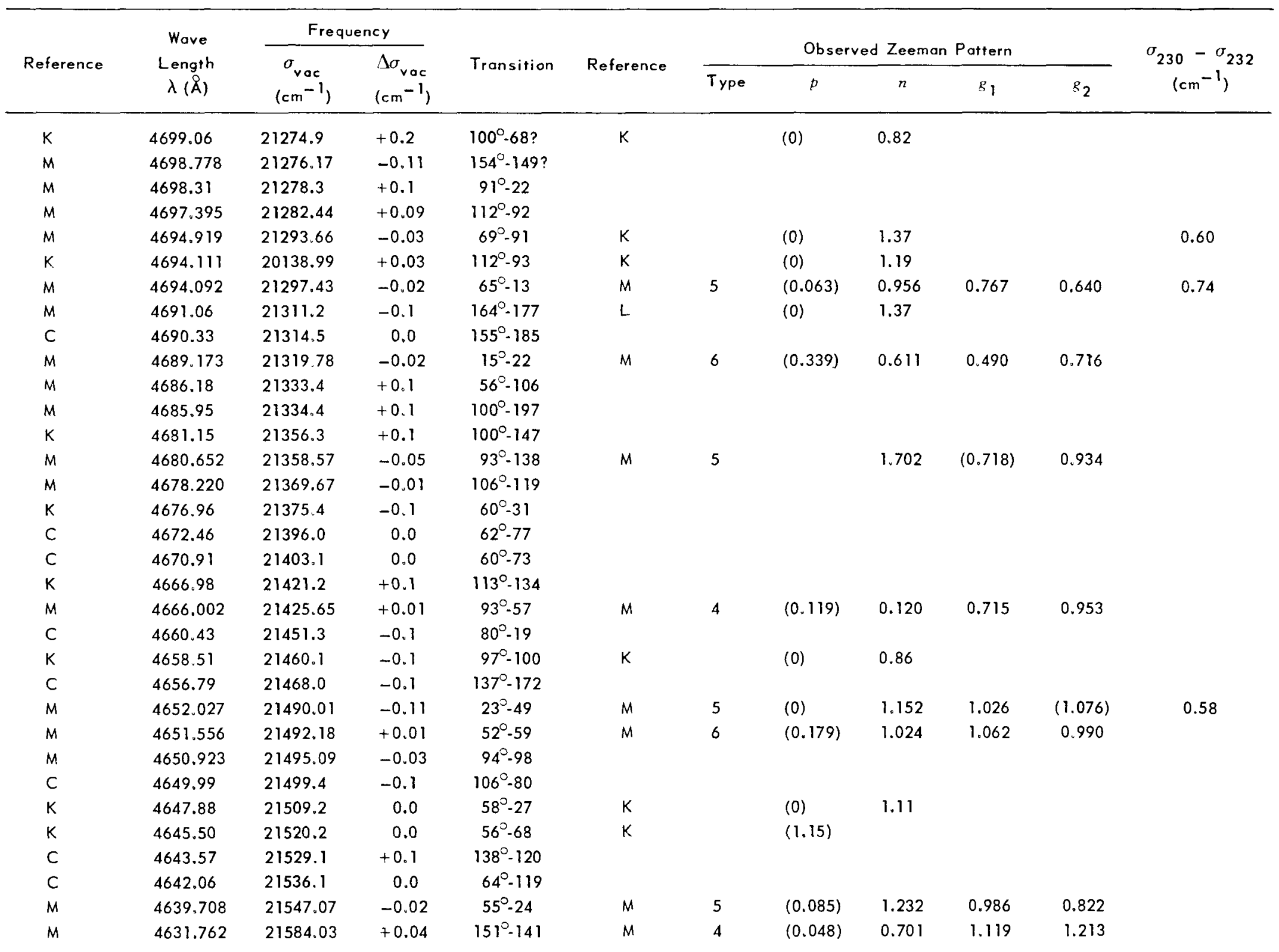


Table 6 (continued)

\begin{tabular}{|c|c|c|c|c|c|c|c|c|c|c|c|}
\hline \multirow{3}{*}{ Reference } & \multirow{3}{*}{$\begin{array}{c}\text { Wave } \\
\text { Length } \\
\lambda(\AA)\end{array}$} & \multicolumn{2}{|c|}{ Frequency } & \multirow{3}{*}{ Transition } & \multirow{3}{*}{ Reference } & \multirow{2}{*}{\multicolumn{5}{|c|}{ Observed Zeeman Pattern }} & \multirow{3}{*}{$\begin{array}{c}\sigma_{230}-\sigma_{232} \\
\left(\mathrm{~cm}^{-1}\right)\end{array}$} \\
\hline & & \multirow{2}{*}{$\begin{array}{c}\sigma_{\mathrm{vac}} \\
\left(\mathrm{cm}^{-1}\right)\end{array}$} & \multirow{2}{*}{$\begin{array}{c}\Delta \sigma_{\mathrm{vac}} \\
\left(\mathrm{cm}^{-1}\right)\end{array}$} & & & & & & & & \\
\hline & & & & & & Type & $p$ & $n$ & $g_{1}$ & $g_{2}$ & \\
\hline K & 4630.363 & 21590.55 & +0.02 & $137^{\circ} \cdot 173$ & K & & $(0)$ & 1.12 & & & \\
\hline M & 4629.772 & 21593.29 & -0.07 & $112^{\circ}-53$ & & & & & & & \\
\hline$M$ & 4628.559 & 21598.97 & -0.11 & $110^{\circ}-87 ?$ & & & & & & & \\
\hline$M$ & 4627.65 & 21603.2 & -0.1 & $135^{\circ} \cdot 106$ & & & & & & & \\
\hline \multirow[t]{2}{*}{ M } & 4626.621 & 21607.99 & -0.06 & $60^{\circ}-114$ & & & & & & & \\
\hline & & & +0.19 & $105^{\circ}-118 ?$ & & & & & & & \\
\hline$K$ & 4625.043 & 21615.29 & +0.05 & $154^{\circ}-150$ & $L$ & & $(0)$ & 0.98 & & & \\
\hline K & 4624.125 & 21619.68 & +0.09 & $97^{\circ}-101$ & K & & $(0.23)$ & 1.01 & & & \\
\hline$M$ & 4623.889 & 21620.76 & -0.01 & $110^{\circ}-88$ & $M$ & $6^{\star}$ & & & & & \\
\hline$M$ & 4620.72 & 21635.6 & +0.1 & $57^{\circ} \cdot 107$ & & & & & & & \\
\hline c & 4620.25 & 21637.8 & 0.0 & $99^{\circ} \cdot 145$ & & & & & & & \\
\hline$M$ & 4619.562 & 21641.01 & -0.05 & $152^{\circ} \cdot 147$ & $K$ & & $(0)$ & 1.29 & & & \\
\hline \multirow[t]{2}{*}{$M, K$} & 4619.480 & 21641.42 & -0.03 & $78^{\circ}-94$ & & & & & & & \\
\hline & & & -0.09 & $131^{\circ} .99$ & L & & (0) & 0.80 & & & \\
\hline$M$ & 4612.54 & 21674.0 & +0.1 & $53^{\circ}-62$ & $\mathrm{~K}$ & & $(0.46)$ & & & & \\
\hline$M$ & 4611.842 & 21677.24 & -0.02 & $95^{\circ}-99$ & $M$ & 6 & $(1.056)$ & 1.076 & 0.913 & 1.217 & \\
\hline \multirow[t]{2}{*}{$K$} & 4609.378 & 21688.85 & -0.01 & $98^{\circ}-65$ & $K$ & & $(0.07)$ & 0.39 & & & \\
\hline & & & & & $M$ & 4 & $(0.071)$ & 0.447 & 0.817 & 0.965 & \\
\hline C & 4607.95 & 21695.6 & +0.2 & $91^{\circ}-57 ?$ & & & & & & & \\
\hline \multirow[t]{2}{*}{$M$} & 4606.506 & 21702.37 & -0.02 & $55^{\circ}-65$ & $M$ & $7 b$ & $(0)$ & 0.986 & 0.986 & 0.986 & \\
\hline & & & +0.14 & $33^{\circ}-16 ?$ & & & & & & & \\
\hline$c$ & 4604.17 & 21713.4 & -0.1 & $153^{\circ} \cdot 148$ & & & & & & & \\
\hline \multirow[t]{2}{*}{$M$} & 4603.929 & 21714.51 & 0.00 & $20^{\circ}-31$ & & & & & & & \\
\hline & & & +0.04 & $27^{\circ}-89$ & & & & & & & \\
\hline$C$ & 4603.38 & 21717.1 & -0.1 & $61^{\circ} .75$ & & & & & & & \\
\hline$M$ & 4602.884 & 21719.44 & 0.00 & $32^{\circ}-15$ & $M$ & 6 & $(0.542)$ & 0.980 & 1.162 & 0.799 & \\
\hline$c$ & 4601.96 & 21723.8 & 0.0 & $110^{\circ}-129$ & & & & & & & \\
\hline
\end{tabular}


Table 6 (continued)

\begin{tabular}{|c|c|c|c|c|c|c|c|c|c|c|c|}
\hline \multirow{3}{*}{ Reference } & \multirow{3}{*}{$\begin{array}{c}\text { Wave } \\
\text { Length } \\
\lambda(\AA)\end{array}$} & \multicolumn{2}{|c|}{ Frequency } & \multirow{3}{*}{ Transition } & \multirow{3}{*}{ Reference } & \multirow{2}{*}{\multicolumn{5}{|c|}{ Observed Zeeman Pattern }} & \multirow{3}{*}{$\begin{array}{c}\sigma_{230}-\sigma_{232} \\
\left(\mathrm{~cm}^{-1}\right)\end{array}$} \\
\hline & & & $\Lambda \sigma$ & & & & & & & & \\
\hline & & $\left(\mathrm{cm}^{-1}\right)$ & $\left(\mathrm{cm}^{-1}\right)$ & & & Type & $p$ & $n$ & $g_{1}$ & $g_{2}$ & \\
\hline \multirow[t]{2}{*}{$M$} & 4596.933 & 21747.54 & -0.02 & $27^{\circ}-42$ & & & & & & & \\
\hline & & & -0.07 & $143^{\circ}-128$ & & & & & & & \\
\hline $\mathrm{C}$ & 4594.01 & 21761.4 & +0.1 & $129^{\circ} \cdot 101$ & & & & & & & \\
\hline K & 4593.559 & 21763.54 & +0.07 & $83^{\circ}-95$ & & & & & & & \\
\hline K & 4589.84 & 21781.2 & 0.0 & $155^{\circ} \cdot 151$ & & & & & & & \\
\hline M & 4589.670 & 21781.95 & 0.00 & $133^{\circ}-143$ & & & & & & & \\
\hline$M$ & 4589.119 & 21784.57 & -0.01 & $98^{\circ}-143$ & & & & & & & \\
\hline C & 4588.25 & 21788.7 & -0.3 & $58^{\circ}-28 ?$ & K & & $(0)$ & 1.01 & & & \\
\hline$M$ & 4587.77 & 21791.0 & 0.0 & $133^{\circ}-104$ & & & & & & & \\
\hline M & 4587.22 & 21793.6 & 0.0 & $98^{\circ}-104$ & & & & & & & \\
\hline K & 4586.637 & 21796.38 & -0.03 & $137^{\circ}-114$ & $\mathrm{~K}$ & & $(0)$ & 1.03 & & & \\
\hline C & 4586.07 & 21799.1 & 0.0 & $65^{\circ} .81$ & & & & & & & \\
\hline$M$ & 4584.376 & 21807.13 & -0.03 & $55^{\circ}-104$ & $M$ & 5 & & 1.156 & $(0.982)$ & 1.031 & \\
\hline$K$ & 4582.79 & 21814.7 & 0.0 & $41^{\circ}-55$ & $K$ & & $(0.16)$ & & & & \\
\hline$K$ & 4581.595 & 21820.36 & -0.10 & $136^{\circ}-148 ?$ & $K$ & & (0) & 1.01 & & & \\
\hline$M$ & 4581.250 & 21821.99 & 0.00 & $102^{\circ} \cdot 72$ & & & & & & & \\
\hline$K$ & 4580.78 & 21824.2 & -0.1 & $101^{\circ} \cdot 108$ & & & & & & & \\
\hline $\mathrm{C}$ & 4579.83 & 21828.8 & +0.1 & $105^{\circ} \cdot 156$ & & & & & & & \\
\hline$M$ & 4579.286 & 21831.34 & +0.06 & $19^{\circ}-27$ & $M$ & 6 & & 0.823 & 0.643 & $(1.003)$ & \\
\hline$M$ & 4579.01 & 21832.7 & 0.0 & $64^{\circ}-120$ & & & & & & & \\
\hline \multirow[t]{2}{*}{$M$} & 4575.427 & 21849.78 & -0.04 & $135^{\circ} \cdot 147$ & $M$ & 6 & & 0.908 & 0.793 & 1.031 & \\
\hline & & & +0.20 & $26^{\circ}-41 ?$ & K & & $(1.06)$ & 1.01 & & & \\
\hline$M$ & 4575.246 & 21850.64 & -0.02 & $24^{\circ}-49$ & $M$ & $7 b$ & (0) & 1.080 & 1.080 & 1.080 & \\
\hline$M$ & 4573.703 & 21858.00 & 0.00 & $93^{\circ}-58$ & & & & & & & \\
\hline$C$ & 4572.92 & 21861.8 & +0.2 & $140^{\circ} \cdot 124 ?$ & & & & & & & \\
\hline$M$ & 4570.629 & 21872.70 & -0.02 & $22^{\circ} \cdot 78$ & & & & & & & \\
\hline$M$ & 4569.024 & 21880.38 & 0.00 & $17^{\circ}-4$ & & & & & & & \\
\hline$M$ & 4567.242 & 21888.92 & -0.03 & $107^{\circ}-81$ & & & & & & & \\
\hline$M$ & 4566.645 & 21891.80 & -0.01 & $33^{\circ}-2$ & $M$ & 4 & $(0.132)$ & 0.853 & 0.986 & 1.251 & \\
\hline$M$ & 4566.249 & 21893.70 & -0.01 & $10^{\circ}-19$ & & & & & & & \\
\hline$M$ & 4564.183 & 21903.61 & 0.00 & $54^{\circ}-100$ & $M$ & 4 & & 0.890 & $(1.088)$ & 1.031 & \\
\hline$M$ & 4563.658 & 21906.11 & -0.09 & $60^{\circ}-74$ & & & & & & & \\
\hline$K$ & 4563.308 & 21907.81 & -0.15 & $163^{\circ}-186$ & L & & (0) & 1.12 & & & \\
\hline
\end{tabular}


Table 6 (continued)

\begin{tabular}{|c|c|c|c|c|c|c|c|c|c|c|c|}
\hline \multirow{3}{*}{ Reference } & \multirow{3}{*}{$\begin{array}{c}\text { Wave } \\
\text { Length } \\
\lambda(\AA)\end{array}$} & \multicolumn{2}{|c|}{ Frequency } & \multirow{3}{*}{ Transition } & \multirow{3}{*}{ Reference } & \multirow{2}{*}{\multicolumn{5}{|c|}{ Observed Zeeman Pattern }} & \multirow{3}{*}{$\begin{array}{c}\sigma_{230}-\sigma_{232} \\
\left(\mathrm{~cm}^{-1}\right)\end{array}$} \\
\hline & & \multirow{2}{*}{$\begin{array}{c}\sigma_{\mathrm{vac}} \\
\left(\mathrm{cm}^{-1}\right)\end{array}$} & \multirow{2}{*}{$\begin{array}{l}\Delta \sigma_{\text {vac }} \\
\left(\mathrm{cm}^{-1}\right)\end{array}$} & & & & & & & & \\
\hline & & & & & & Type & $p$ & $n$ & $g_{1}$ & $g_{2}$ & \\
\hline $\mathrm{K}$ & 4562.59 & 21911.3 & +0.2 & $73^{\circ}-52 ?$ & & & & & & & \\
\hline$M$ & 4556.809 & 21939.05 & +0.03 & $2^{\circ}-6$ & & & & & & & \\
\hline $\mathrm{C}$ & 4554.81 & 21948.7 & +0.1 & $144^{\circ}-129$ & & & & & & & \\
\hline K & 4554.07 & 21952.2 & +0.1 & $34^{\circ}-53$ & & & & & & & \\
\hline K & 4553.851 & 21953.31 & -0.17 & $154^{\circ}-184 ?$ & & & & & & & \\
\hline$M$ & 4553.038 & 21957.20 & +0.05 & $102^{\circ}-73$ & $L$ & & $(0)$ & 0.96 & & & \\
\hline$M$ & 4552.31 & 21960.7 & +0.1 & $27^{\circ}-43$ & & & & & & & \\
\hline$M$ & 4546.84 & 21987.1 & -0.1 & $134^{\circ}-145$ & & & & & & & \\
\hline $\mathrm{K}$ & 4545.849 & 21991.95 & -0.13 & $78^{\circ}-18 ?$ & & & & & & & \\
\hline M & 4545.264 & 21994.76 & +0.06 & $75^{\circ} .93$ & & & & & & & \\
\hline$M$ & 4544.518 & 21998.38 & +0.03 & $51^{\circ}-56$ & $M$ & 6 & $(0.197)$ & 0.816 & 0.853 & 0.781 & \\
\hline$M$ & 4543.208 & 22004.71 & +0.03 & $132^{\circ}-100$ & $\mathrm{~K}$ & & $(0)$ & 0.75 & & & \\
\hline $\mathrm{K}$ & 4541.636 & 22012.35 & -0.07 & $101^{\circ}-69$ & $k$ & & $(0.13)$ & 1.85 & & & \\
\hline$M$ & 4541.209 & 22014.40 & -0.02 & $52^{\circ}-61$ & & & & & & & \\
\hline$M$ & 4540.425 & 22018.22 & -0.01 & $100^{\circ} \cdot 107$ & $M$ & 6 & $(0.565)$ & 0.941 & 0.862 & 1.023 & \\
\hline$c$ & 4538.29 & 22028.6 & 0.0 & $68^{\circ} \cdot 89$ & & & & & & & \\
\hline$M$ & 4538.18 & 22029.1 & 0.0 & $20^{\circ}-32$ & & & & & & & \\
\hline \multirow[t]{2}{*}{$M, K$} & 4537.079 & 22034.45 & -0.05 & $135^{\circ}-169$ & $M$ & 5 & $(0.048)$ & 1.366 & 0.776 & 0.884 & 0.45 \\
\hline & & & -0.04 & $136^{\circ}-108$ & & & & & & & \\
\hline $\mathrm{K}$ & 4535.72 & 22041.1 & -0.1 & $38^{\circ}-3$ & & & & & & & \\
\hline$M$ & 4534.129 & 22048.79 & -0.02 & $132^{\circ}-166$ & $M$ & 4 & $(0.050)$ & 0.373 & 0.927 & 0.826 & 0.47 \\
\hline$M$ & 4533.309 & 22052.78 & -0.01 & $96^{\circ}-141$ & $M$ & 5 & & 1.359 & $(1.173)$ & 1.215 & \\
\hline $\mathrm{K}$ & 4532.43 & 22057.1 & 0.0 & $95^{\circ}-62$ & & & & & & & \\
\hline$M$ & 4532.264 & 22057.84 & -0.08 & $98^{\circ}-66$ & $M$ & 4 & & 0.496 & 0.843 & $(1.003)$ & \\
\hline$M$ & 4531.721 & 22060.48 & -0.04 & $99^{\circ}-106$ & $\mathrm{~K}$ & & $(0.54)$ & 1.07 & & & \\
\hline$K$ & 4531.21 & 22063.0 & -0.1 & $54^{\circ}-101$ & & & & & & & \\
\hline$M$ & 4529.487 & 22071.39 & -0.06 & $55^{\circ}-66$ & $M$ & $7 b$ & $(0)$ & 0.995 & 0.995 & 0.995 & \\
\hline $\mathrm{K}$ & 4528.99 & 22073.8 & -0.1 & $94^{\circ}-140$ & & & & & & & \\
\hline $\mathrm{K}$ & 4528.421 & 22076.58 & -0.06 & $134^{\circ}-146$ & & & & & & & \\
\hline$M$ & 4527.733 & 22079.91 & -0.08 & $137^{\circ}-174$ & & & & & & & \\
\hline$K$ & 4526.05 & 22088.1 & -0.1 & $113^{\circ}-92$ & $K$ & & $(0.69)$ & 0.81 & & & \\
\hline$K$ & 4525.100 & 22092.78 & -0.04 & $97^{\circ} \cdot 102$ & $K$ & & $(0.20)$ & 1.00 & & & \\
\hline$M$ & 4524.84 & 22094.1 & +0.1 & $72^{\circ}-15$ & $M$ & 5 & & 1.292 & 0.992 & 0.792 & \\
\hline
\end{tabular}


Table 6 (cont inued)

\begin{tabular}{|c|c|c|c|c|c|c|c|c|c|c|c|}
\hline \multirow{3}{*}{ Reference } & \multirow{3}{*}{$\begin{array}{c}\text { Wave } \\
\text { Length } \\
\lambda(\AA)\end{array}$} & \multicolumn{2}{|c|}{ Frequency } & \multirow{3}{*}{ Transition } & \multirow{3}{*}{ Reference } & \multirow{2}{*}{\multicolumn{5}{|c|}{ Observed Zeeman Pattern }} & \multirow{3}{*}{$\begin{array}{c}\sigma_{230}-\sigma_{232} \\
\left(\mathrm{~cm}^{-1}\right)\end{array}$} \\
\hline & & $\sigma_{\text {vac }}$ & $\Delta \sigma_{\text {yoc }}$ & & & & & & & & \\
\hline & & $\left(\mathrm{cm}^{-1}\right)$ & $\left(\mathrm{cm}^{-1}\right)$ & & & Type & $p$ & $n$ & $g_{1}$ & $g_{2}$ & \\
\hline$K$ & 4524.57 & 22095.4 & +0.1 & $39^{\circ}-3$ & & & & & & & \\
\hline$K$ & 4521.47 & 22110.5 & 0.0 & $8^{\circ}-18$ & & & & & & & \\
\hline C & 4520.00 & 22117.7 & +0.1 & $67^{\circ}-87$ & & & & & & & \\
\hline$K$ & 4519.761 & 22118.88 & -0.09 & $17^{\circ}-25$ & $K$ & & $(0.19)$ & 1.15 & & & \\
\hline$M$ & 4519.273 & 22121.25 & +0.01 & $108^{\circ}-123$ & & & & & & & \\
\hline$K$ & 4518.651 & 22124.32 & -0.05 & $155^{\circ}-152$ & $\mathrm{~K}$ & & $(0)$ & $1: 27$ & & & \\
\hline \multirow[t]{4}{*}{$M$} & 4517.046 & 22132.18 & -0.06 & $15^{\circ}-59$ & & & & & & & \\
\hline & & & +0.04 & $25^{\circ}-41$ & & & & & & & \\
\hline & & & -0.20 & $58^{\circ}-110 ?$ & $K$ & & $(0)$ & 0.85 & & & \\
\hline & & & & & $M$ & 4 & & 0.742 & 1.077 & $(0.981)$ & \\
\hline M & 4515.979 & 22137.38 & -0.01 & $138^{\circ}-179$ & $L$ & & $(0)$ & 1.21 & & & \\
\hline$C$ & 4515.11 & 22141.7 & 0.0 & $105^{\circ}-119$ & & & & & & & \\
\hline$M$ & & & -0.13 & $142^{\circ}-161$ & & & & & & & \\
\hline$M$ & 4510.535 & 22164.12 & +0.01 & $132^{\circ}-101$ & $M$ & 5 & & 0.992 & $(0.931)$ & 0.913 & \\
\hline $\mathrm{K}$ & 4509.98 & 22166.9 & 0.0 & $26^{\circ}-87$ & & & & & & & \\
\hline$c$ & 4509.58 & 22168.8 & 0.0 & $53^{\circ}-63$ & & & & & & & \\
\hline$M$ & 4508.651 & 22173.36 & -0.03 & $133^{\circ}-167$ & & & & & & & \\
\hline$c$ & 4508.61 & 22173.6 & 0.0 & $141^{\circ}-161$ & & & & & & & \\
\hline$M$ & 4508.170 & 22175.73 & -0.10 & $154^{\circ}-172$ & & & & & & & \\
\hline \multirow[t]{2}{*}{$k$} & 4496.323 & 22234.18 & -0.02 & $164^{\circ}-178$ & $\mathrm{~K}$ & & $(0)$ & 1.24 & & & \\
\hline & & & -0.01 & $19^{\circ}-70$ & & & & & & & \\
\hline$c$ & 4495.55 & 22238.0 & 0.0 & $24^{\circ}-86$ & & & & & & & \\
\hline$M$ & 4495.49 & 22238.3 & 0.0 & $133^{\circ} \cdot 105$ & & & & & & & \\
\hline$M$ & 4495.247 & 22239.48 & -0.05 & $61^{\circ} \cdot 33$ & & & & & & & \\
\hline $\mathrm{K}$ & 4494.971 & 22240.88 & -0.01 & $98^{\circ} \cdot 105$ & $K$ & & $(0.39)$ & 0.77 & & & \\
\hline$c$ & 4494.68 & 22242.3 & 0.0 & $67^{\circ}-129$ & & & & & & & \\
\hline$K$ & 4493.65 & 22247.4 & 0.0 & $99^{\circ}-68$ & & & & & & & \\
\hline $\mathrm{C}$ & 4491.36 & 22258.9 & +0.1 & $58^{\circ}-111 ?$ & & & & & & & \\
\hline
\end{tabular}


Table 6 (cont inved)

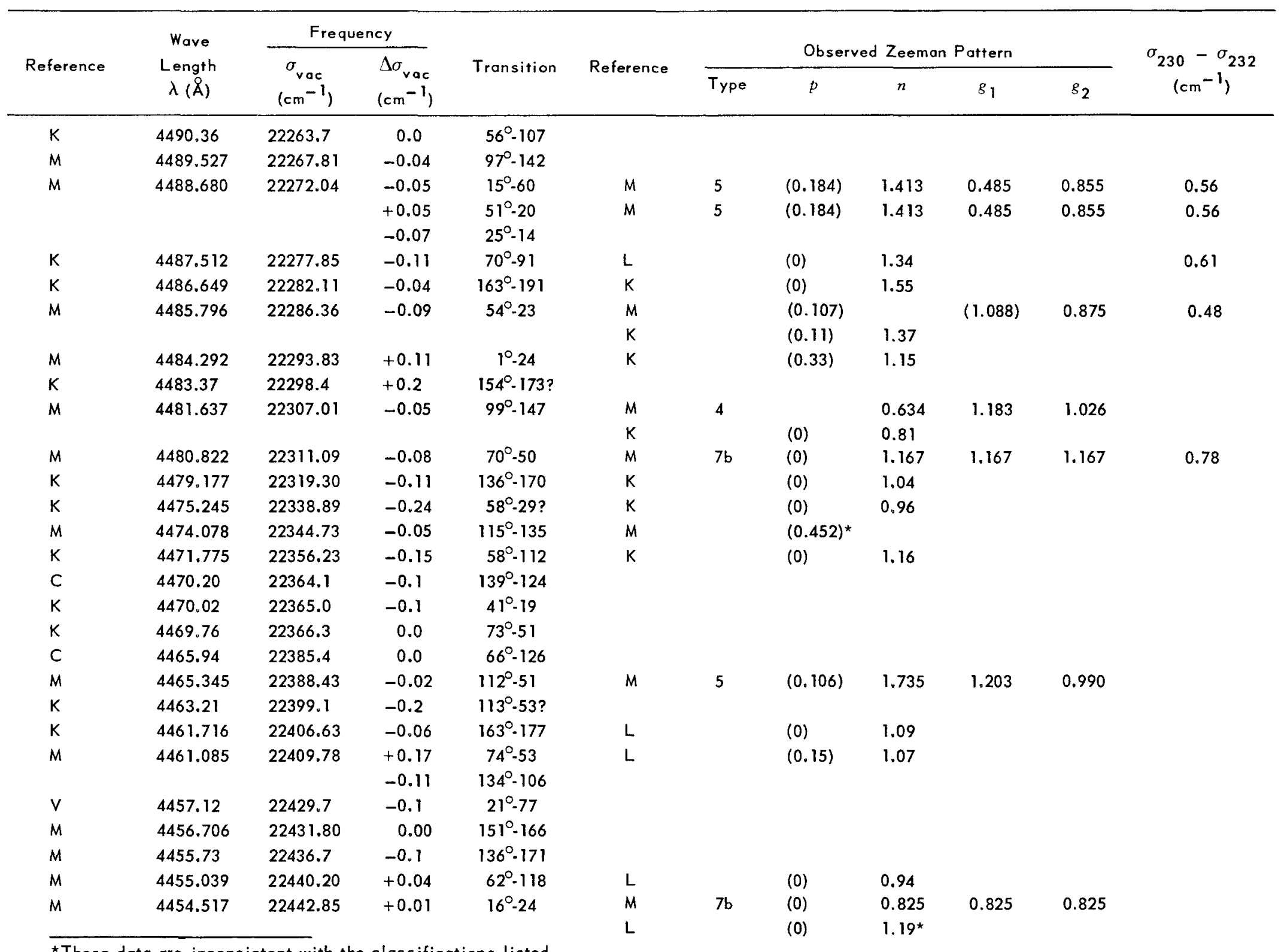


Table 6 (continued)

\begin{tabular}{|c|c|c|c|c|c|c|c|c|c|c|c|}
\hline \multirow[b]{2}{*}{ Reference } & \multirow{2}{*}{$\begin{array}{c}\text { Wave } \\
\text { Length } \\
\lambda(\AA)\end{array}$} & \multicolumn{2}{|c|}{ Frequency } & \multirow[b]{2}{*}{ Transition } & \multirow[b]{2}{*}{ Reference } & \multicolumn{5}{|c|}{ Observed Zeeman Pattern } & \multirow[b]{2}{*}{$\begin{array}{c}\sigma_{230}-\sigma_{232} \\
\left(\mathrm{~cm}^{-1}\right)\end{array}$} \\
\hline & & $\begin{array}{c}\sigma_{\text {vac }} \\
\left(\mathrm{cm}^{-1}\right)\end{array}$ & $\begin{array}{l}\Delta \sigma_{\text {vac }} \\
\left(\mathrm{cm}^{-1}\right)\end{array}$ & & & Type & Ooser & d Leem & oftern & $g_{2}$ & \\
\hline c & 4453.19 & 22449.5 & +0.1 & $25^{\circ}-87$ & & & & & & & \\
\hline M & 4451.056 & 22460.28 & +0.10 & $102^{\circ}-74 ?$ & & & & & & & \\
\hline M & 4447.845 & 22476.51 & -0.05 & $32^{\circ}-1$ & M & 4 & $(0.476)$ & 0.686 & 1.163 & 2.116 & \\
\hline $\mathrm{k}$ & 4445.916 & 22486.26 & -0.07 & $100^{\circ}-108$ & K & & (0) & 0.88 & & & \\
\hline M & 4444.979 & 22490.98 & 0.00 & $153^{\circ}-149$ & & & & & & & \\
\hline M & 4441.90 & 22506.6 & +0.1 & $65^{\circ}-123$ & & & & & & & \\
\hline M & 4440.872 & 22511.81 & +0.02 & $73^{\circ}-17$ & M & 5 & $(0.128)$ & 1.598 & 1.223 & 0.972 & \\
\hline c & 4440.56 & 22513.4 & +0.1 & $66^{\circ}-13$ & $\mathrm{~K}$ & & $(0.28)$ & & & & \\
\hline \multirow[t]{2}{*}{$M$} & 4439.128 & 22520.65 & 0.00 & $97^{\circ}-103$ & M & 6 & $(0.536)$ & & 0.985 & 0.831 & 0.35 \\
\hline & & & & & L & & $(0.48)$ & 0.88 & & & \\
\hline $\mathrm{k}$ & 4437.73 & 22527.7 & -0.1 & $35^{\circ}-53$ & & & & & & & \\
\hline M & 4436.558 & 22533.69 & +0.01 & $135^{\circ}-107$ & M & 4 & $(0.113)$ & 0 & 0.791 & 1.017 & \\
\hline M & 4436.298 & 22534.99 & -0.06 & $131^{\circ}-141$ & K & & $(0.57)$ & & & & \\
\hline M & 4436.046 & 22536.29 & +0.02 & $54^{\circ}-102$ & $\mathrm{~K}$ & & $(0)$ & 1.00 & & & \\
\hline $\mathrm{K}$ & 4434.84 & 22542.4 & -0.1 & $52^{\circ}-98$ & $\mathrm{~K}$ & & (0) & 1.16 & & & \\
\hline $\mathrm{k}$ & 4434.354 & 22544.89 & -0.11 & $136^{\circ}-109 ?$ & K & & (0) & 0.89 & & & \\
\hline c & 4434.05 & 22546.4 & 0.0 & $6^{\circ}-16$ & & & & & & & \\
\hline c & 4433.49 & 22549.3 & +0.1 & $55^{\circ}-25$ & & & & & & & \\
\hline K & 4432.970 & 22551.93 & -0.03 & $95^{\circ}-63$ & K & & (0) & 0.86 & & & \\
\hline$K, M$ & 4429.270 & 22570.76 & -0.05 & $95^{\circ}-141$ & & & & & & & \\
\hline \multirow[t]{2}{*}{$\mathrm{K}$} & 4427.662 & 22578.96 & -0.03 & $152^{\circ}-148$ & L & & (0) & 1.39 & & & \\
\hline & & & +0.02 & $20^{\circ} \cdot 75$ & & & & & & & \\
\hline K & 4426.20 & 22586.4 & 0.0 & $79^{\circ}-94$ & & & & & & & \\
\hline M & 4423.945 & 22597.91 & -0.10 & $136^{\circ}-149 ?$ & & & & & & & \\
\hline C & 4423.25 & 22601.5 & -0.1 & $107^{\circ}-159 ?$ & & & & & & & \\
\hline M & 4422.783 & 22603.87 & -0.05 & $67^{\circ}-14$ & & & & & & & \\
\hline K & 4421.551 & 22610.17 & +0.03 & $25^{\circ}-49$ & $\mathrm{~K}$ & & $(0.15)$ & & & & \\
\hline M & 4420.80 & 22614.0 & -0.2 & $57^{\circ}-109 ?$ & & & & & & & \\
\hline c & 4419.31 & 22621.6 & +0.1 & $141^{\circ}-128$ & & & & & & & \\
\hline K & 4419.002 & 22623.22 & +0.09 & $137^{\circ}-152$ & $\mathrm{~K}$ & & $(0.18)$ & 0.98 & & & \\
\hline K & 4418.658 & 22624.98 & +0.11 & $147^{\circ}-95$ & $\kappa$ & & $(0)$ & 1.91 & & & \\
\hline C & 4418.43 & 22626.1 & +0.1 & $22^{\circ}-36$ & & & & & & & \\
\hline M & 4416.241 & 22637.36 & +0.02 & $132^{\circ} \cdot 102$ & $M$ & 4 & $(0.061)$ & 0.506 & 0.927 & 1.048 & \\
\hline
\end{tabular}


Table 6 (cont inued)

\begin{tabular}{|c|c|c|c|c|c|c|c|c|c|c|c|}
\hline \multirow{3}{*}{ Reference } & \multirow{3}{*}{$\begin{array}{c}\text { Wave } \\
\text { Length } \\
\lambda(\AA)\end{array}$} & \multicolumn{2}{|c|}{ Frequency } & \multirow{3}{*}{ Transition } & \multirow{3}{*}{ Reference } & \multirow{2}{*}{\multicolumn{5}{|c|}{ Observed Zeeman Pattern }} & \multirow{3}{*}{$\begin{array}{c}\sigma_{230}-\sigma_{232} \\
\left(\mathrm{~cm}^{-1}\right)\end{array}$} \\
\hline & & $\sigma_{\text {vac }}$ & $\Delta \sigma_{\text {vac }}$ & & & & & & & & \\
\hline & & $\left(\mathrm{cm}^{-1}\right)$ & $\left(\mathrm{cm}^{-1}\right)$ & & & Type & $p$ & $n$ & $g_{1}$ & $g_{2}$ & \\
\hline$M$ & 4413.378 & 22652.02 & -0.03 & $101^{\circ} \cdot 71$ & & & & & & & \\
\hline$M$ & 4412.897 & 22654.48 & -0.01 & $15^{\circ}-61$ & $\mathrm{~K}$ & & $(0.24)$ & & & & \\
\hline \multirow[t]{2}{*}{ M } & 4412.745 & 22655.29 & +0.01 & $93^{\circ}-59$ & $M$ & 4 & $(0.132)$ & 0.030 & 0.716 & 0.983 & \\
\hline & & & & & $L$ & & $(0)$ & $1.00^{\star}$ & & & \\
\hline$K$ & 4412.535 & 22656.37 & -0.06 & $134^{\circ}-147$ & $K$ & & $(0.70)$ & & & & 0.37 \\
\hline$M$ & 4411.634 & 22661.02 & +0.09 & $61^{\circ}-77$ & & & & & & & \\
\hline$K$ & 4410.475 & 22666.95 & +0.15 & $137^{\circ}-175 ?$ & $L$ & & $(0)$ & 1.31 & & & \\
\hline$K$ & 4408.98 & 22674.6 & +0.2 & $100^{\circ}-69 ?$ & & & & & & & \\
\hline$M$ & 4408.436 & 22677.43 & +0.15 & $18^{\circ}-28$ & $M$ & 6 & $(0.350)$ & 1.250 & 1.366 & 1.136 & \\
\hline$c$ & 4406.97 & 22685.0 & +0.1 & $67^{\circ}-43$ & & & & & & & \\
\hline$K$ & 4404.91 & 22695.6 & -0.1 & $58^{\circ}-72$ & & & & & & & \\
\hline$K$ & 4402.732 & 22706.81 & +0.12 & $82^{\circ}-55 ?$ & K & & $(0.87)$ & & & & \\
\hline$M$ & 4401.986 & 22710.66 & +0.01 & $2^{\circ}-7$ & & & & & & & \\
\hline $\mathrm{K}$ & 4401.634 & 22712.48 & +0.14 & $101^{\circ} \cdot 110$ & $K$ & & $(1.07)$ & 1.02 & & & \\
\hline C & 4401.21 & 22714.7 & 0.0 & $103^{\circ}-118$ & & & & & & & \\
\hline$K$ & 4400.379 & 22718.95 & -0.05 & $33^{\circ}-15$ & $\mathrm{~K}$ & & $(0.27)$ & 0.90 & & & \\
\hline$K$ & 4397.898 & 22731.77 & -0.03 & $56^{\circ}-108$ & $L$ & & $(0.20)$ & $0.72^{\star}$ & & & \\
\hline$M$ & 4396.479 & 22739.11 & -0.02 & $113^{\circ}-52$ & $M$ & 4 & $(0.126)$ & 0.543 & 1.173 & 1.424 & 0.58 \\
\hline$M$ & 4394.899 & 22747.28 & +0.01 & $34^{\circ}-51$ & $M$ & 5 & $(0.063)$ & 1.190 & 0.869 & 0.995 & 0.36 \\
\hline$M$ & 4391.114 & 22766.89 & -0.01 & $51^{\circ}-97$ & M & 5 & $(0.087)$ & 1.469 & 0.856 & 1.031 & 0.39 \\
\hline \multirow[t]{2}{*}{$M$} & 4388.413 & 22780.90 & +0.04 & $10^{\circ}-3$ & & & & & & & \\
\hline & & & -0.01 & $71^{\circ} \cdot 48$ & & & & & & & \\
\hline \multirow[t]{2}{*}{$K$} & 4387.10 & 22787.7 & 0.0 & $154^{\circ} \cdot 174$ & $\mathrm{~K}$ & & $(0.40)$ & & & & \\
\hline & & & -0.1 & $135^{\circ} \cdot 148$ & & & & & & & \\
\hline$C$ & 4387.04 & 22788.0 & -0.1 & $5^{\circ}-46$ & & & & & & & \\
\hline $\mathrm{C}$ & 4385.75 & 22794.7 & +0.1 & $83^{\circ}-55$ & & & & & & & \\
\hline$C$ & 4384.65 & 22800.5 & +0.1 & $18^{\circ} \cdot 70$ & & & & & & & \\
\hline$M$ & 4384.12 & 22803.2 & 0.0 & $58^{\circ} \cdot 31$ & & & & & & & \\
\hline
\end{tabular}


Table 6 (continued)

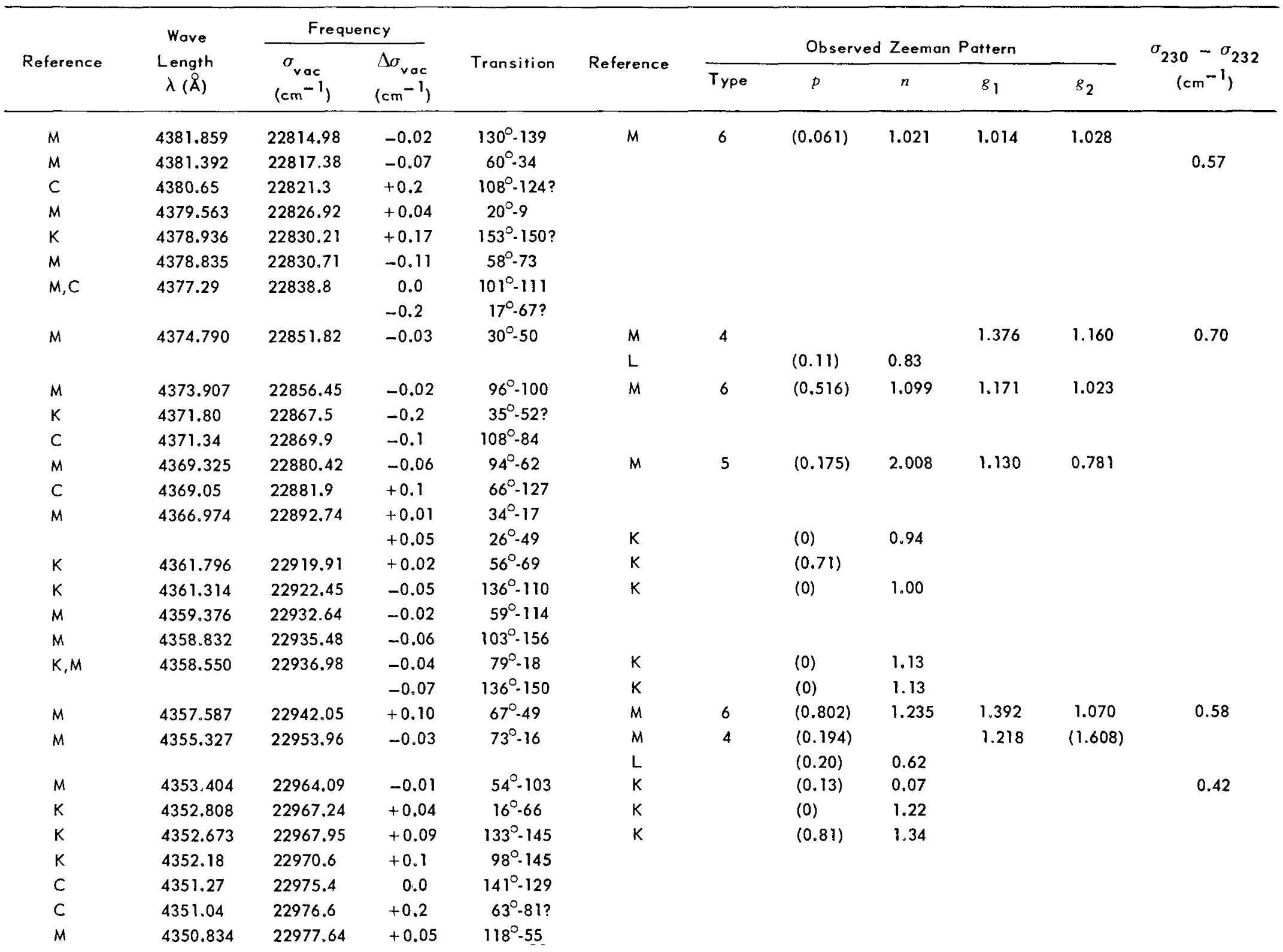


Table 6 (continued)

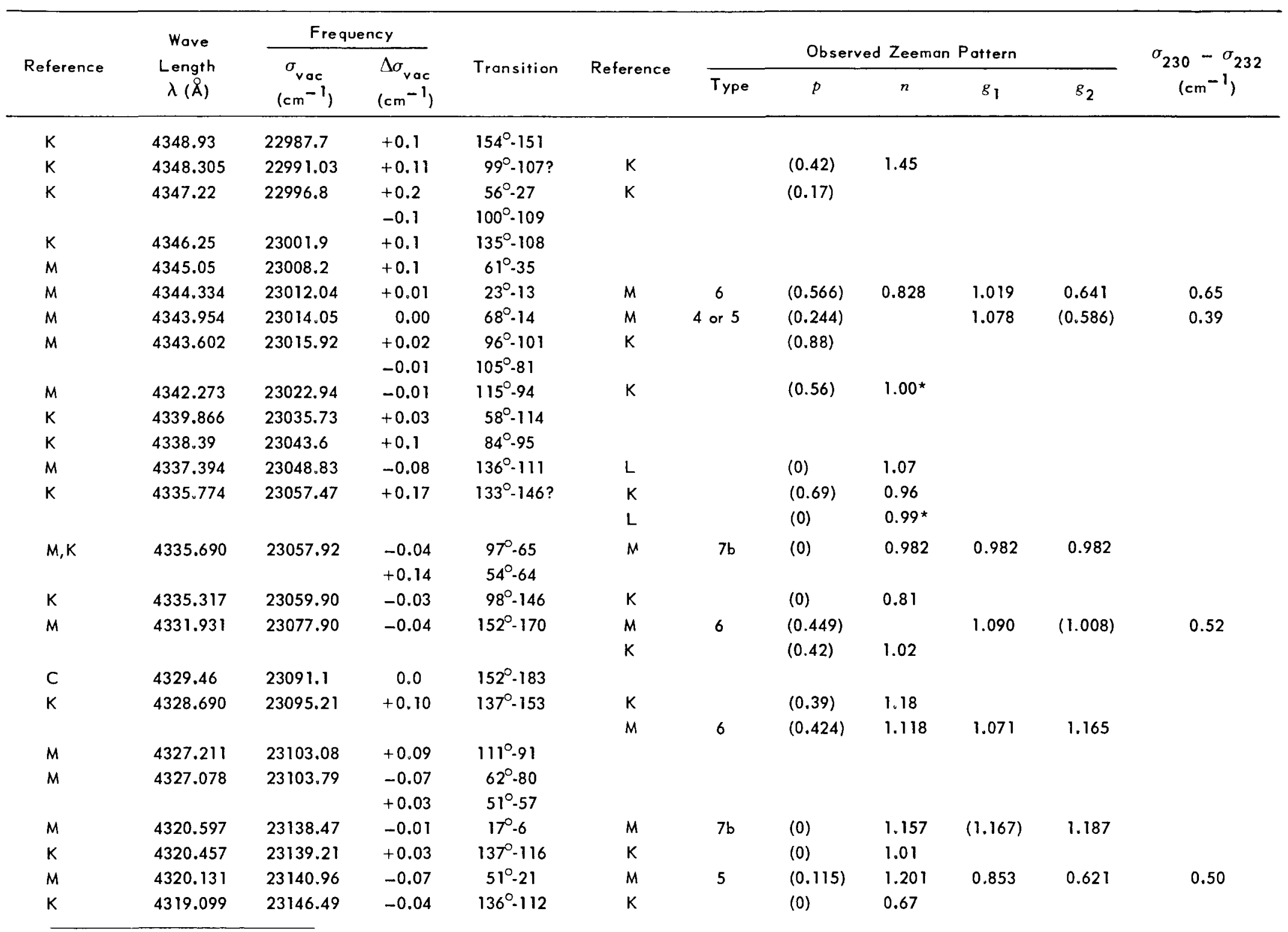

*These data are inconsistent with the classifications listed. 
Table 6 (cont inued)

\begin{tabular}{|c|c|c|c|c|c|c|c|c|c|c|c|}
\hline \multirow{3}{*}{ Reference } & \multirow{3}{*}{$\begin{array}{c}\text { Wave } \\
\text { Length } \\
\lambda(\AA)\end{array}$} & \multicolumn{2}{|c|}{ Frequency } & \multirow{3}{*}{ Transition } & \multirow{3}{*}{ Reference } & \multirow{2}{*}{\multicolumn{5}{|c|}{ Observed Zeeman Pattern }} & \multirow{3}{*}{$\begin{array}{c}\sigma_{230}-\sigma_{232} \\
\left(\mathrm{~cm}^{-1}\right)\end{array}$} \\
\hline & & \multirow{2}{*}{$\begin{array}{c}\sigma_{\text {vac }} \\
\left(\mathrm{cm}^{-1}\right)\end{array}$} & \multirow{2}{*}{$\begin{array}{l}\Delta \sigma_{\text {vac }} \\
\left(\mathrm{cm}^{-1}\right)\end{array}$} & & & & & & & & \\
\hline & & & & & & Type & $p$ & $n$ & $g_{1}$ & $g_{2}$ & \\
\hline$M$ & 4318.300 & 23150.77 & -0.02 & $53^{\circ}-101$ & $M$ & 5 & $(0.143)$ & 1.668 & 0.603 & 0.907 & \\
\hline K & 4317.68 & 23154.1 & +0.1 & $100^{\circ} \cdot 70$ & & & & & & & \\
\hline C & 4317.23 & 23156.5 & 0.0 & $20^{\circ}-34$ & & & & & & & \\
\hline $\mathrm{C}$ & 4316.09 & 23162.6 & -0.1 & $97^{\circ} \cdot 104$ & & & & & & & \\
\hline$M$ & 4315.957 & 23163.34 & 0.00 & $17^{\circ}-68$ & $M$ & 4 & $(0.141)$ & 0.462 & 1.166 & 0.884 & \\
\hline M & 4314.00 & 23173.8 & +0.1 & $130^{\circ}-98 ?$ & & & & & & & \\
\hline M & 4313.314 & 23177.51 & -0.04 & $93^{\circ}-61$ & M & 4 & $(0.109)$ & 0,172 & 0.717 & 0.935 & \\
\hline$k$ & 4310.18 & 23194.4 & $\begin{array}{r}+0.1 \\
0.0\end{array}$ & $\begin{array}{r}39^{\circ}-54 \\
113^{\circ}-51\end{array}$ & & & & & & & \\
\hline \multirow[t]{2}{*}{$M$} & 4309.994 & 23195.37 & +0.01 & $151^{\circ}-142$ & & & & & & & \\
\hline & & & +0.01 & $152^{\circ}-171$ & & & & & & & \\
\hline $\mathrm{K}$ & 4309.22 & 23199.6 & 0.0 & $42^{\circ}-19$ & & & & & & & \\
\hline$K$ & 4306.376 & 23214.87 & 0.00 & $164^{\circ}-179$ & $K$ & & $(0)$ & 1.92 & & & 0.7 \\
\hline$c$ & 4306.14 & 23216.1 & +0.1 & $108^{\circ}-126$ & & & & & & & \\
\hline C & 4305.87 & 23217.6 & +0.2 & $91^{\circ}-96 ?$ & & & & & & & \\
\hline$c$ & 4305.33 & 23220.5 & -0.1 & $111^{\circ}-163$ & & & & & & & \\
\hline $\mathrm{C}$ & 4303.39 & 23231.0 & +0.2 & $59^{\circ}-74 ?$ & & & & & & & \\
\hline $\mathrm{K}$ & 4301.29 & 23242.3 & 0.0 & $56^{\circ}-109$ & & & & & & & \\
\hline \multirow[t]{2}{*}{$M$} & 4300.799 & 23244.96 & -0.03 & $99^{\circ}-148$ & $K$ & & $(0)$ & $0.65^{\star}$ & & & \\
\hline & & & -0.04 & $147^{\circ} \cdot 164$ & $K$ & & (0) & $0.65^{*}$ & & & \\
\hline \multirow[t]{2}{*}{$\mathrm{K}$} & 4299.46 & 23252.2 & +0.1 & $23^{\circ} .41$ & & & & & & & \\
\hline & & & 0.0 & $41^{\circ}-3$ & & & & & & & \\
\hline \multirow[t]{2}{*}{$C, K$} & 4298.835 & 23255.60 & -0.05 & $98^{\circ}-67$ & $K$ & & $(0)$ & 0.73 & & & \\
\hline & & & +0.14 & $139^{\circ}-127 ?$ & & & & & & & \\
\hline $\mathrm{C}$ & 4298.54 & 23257.2 & +0.1 & $82^{\circ}-19$ & & & & & & & \\
\hline $\mathrm{K}$ & 4295.122 & 23275.70 & +0.05 & $101^{\circ}-72$ & $K, L$ & & $(0)$ & 1.31 & & & \\
\hline$K$ & 4295.047 & 23276.11 & +0.01 & $155^{\circ}-154$ & $\mathrm{~K}$ & & $(0)$ & 1.09 & & & \\
\hline K & 4293.07 & 23286.8 & 0.0 & $157^{\circ} \cdot 190$ & & & & & & & \\
\hline$K, C$ & 4291.39 & 23295.9 & +0.1 & $1^{\circ}-25$ & $K$ & & $(0.37)$ & 1.51 & & & \\
\hline $\mathrm{C}$ & 4290.38 & 23301.4 & +0.1 & $22^{\circ} \cdot 80$ & & & & & & & \\
\hline
\end{tabular}

* These data are inconsistent with the classifications listed. 
Table 6 (continued)

\begin{tabular}{|c|c|c|c|c|c|c|c|c|c|c|c|}
\hline \multirow{3}{*}{ Reference } & \multirow{3}{*}{$\begin{array}{c}\text { Wove } \\
\text { Length } \\
\lambda(\AA)\end{array}$} & \multicolumn{2}{|c|}{ Frequency } & \multirow{3}{*}{ Transition } & \multirow{3}{*}{ Reference } & \multirow{2}{*}{\multicolumn{5}{|c|}{ Observed Zeeman Pattern }} & \multirow{3}{*}{$\begin{array}{c}\sigma_{230}-\sigma_{232} \\
\left(\mathrm{~cm}^{-l}\right)\end{array}$} \\
\hline & & \multirow{2}{*}{$\begin{array}{c}\sigma_{\mathrm{vac}} \\
\left(\mathrm{cm}^{-1}\right)\end{array}$} & \multirow{2}{*}{$\begin{array}{l}\Delta \sigma_{\text {vac }} \\
\left(\mathrm{cm}^{-1}\right)\end{array}$} & & & & & & & & \\
\hline & & & & & & Type & $p$ & $n$ & $g_{1}$ & $g_{2}$ & \\
\hline C & 4290.17 & 23302.6 & +0.1 & $137^{\circ} \cdot 176$ & & & & & & & \\
\hline$M$ & 4288.056 & 23314.05 & 0.00 & $100^{\circ}-71$ & $M$ & 4 & $(0.155)$ & 0.086 & 0.859 & 1.167 & \\
\hline C & 4286.45 & 23322.8 & -0.1 & $35^{\circ} .51$ & & & & & & & \\
\hline K & 4285.185 & 23329.68 & +0.04 & $163^{\circ}-178$ & K & & $(0)$ & 0.95 & & & \\
\hline K & 4284.979 & 23330.80 & -0.04 & $154^{\circ} .152$ & $L$ & & $(0)$ & 0.81 & & & \\
\hline$\checkmark$ & 4284.50 & 23333.4 & +0.1 & $116^{\circ} \cdot 54$ & & & & & & & \\
\hline \multirow[t]{2}{*}{ M } & 4283.525 & 23338.72 & -0.01 & $131^{\circ} \cdot 100$ & $M$ & & $(0.033)$ & & $(1.086)$ & 1.020 & \\
\hline & & & & & $L$ & & $(0)$ & 1.25 & & & \\
\hline \multirow[t]{3}{*}{ K } & 4282.378 & 23344.96 & -0.02 & $83^{\circ} \cdot 19$ & & & & & & & \\
\hline & & & -0.01 & $61^{\circ}-79$ & & & & & & & \\
\hline & & & -0.11 & $107^{\circ}-84$ & & & & & & & \\
\hline$M$ & 4282.044 & 23346.79 & -0.02 & $93^{\circ}-139$ & $M$ & 5 & $(0.156)$ & 2.127 & 0.720 & 1.032 & 0.33 \\
\hline$M$ & 4281.425 & 23350.16 & -0.02 & $74^{\circ} \cdot 17$ & $K$ & & $(0)$ & 1.26 & & & \\
\hline$M$ & 4281.072 & 23352.09 & +0.01 & $68^{\circ}-49$ & $M$ & $7 b$ & $(0)$ & 1.073 & 1.073 & 1.073 & 0.36 \\
\hline \multirow[t]{2}{*}{$V, K$} & 4280.26 & 23356.5 & -0.2 & $78^{\circ}-93$ & $K$ & & $(0)$ & 1.18 & & & \\
\hline & & & 0.0 & $152^{\circ} \cdot 149$ & $K$ & & $(0)$ & 1.18 & & & \\
\hline$K$ & 4278.53 & 23366.0 & 0.0 & $121^{\circ} \cdot 95$ & & & & & & & \\
\hline$M$ & 4277.322 & 23372.56 & -0.01 & $24^{\circ}-13$ & $M$ & 6 & $(0.641)$ & 0.851 & 1.069 & 0.640 & 0.55 \\
\hline \multirow[t]{2}{*}{$\mathrm{K}$} & 4277.03 & 23374.2 & 0.0 & $53^{\circ} \cdot 23$ & & & & & & & \\
\hline & & & -0.1 & $100^{\circ}-110$ & & & & & & & \\
\hline \multirow[t]{3}{*}{$\mathrm{K}$} & 4276.973 & 23374.46 & -0.02 & $95^{\circ} \cdot 100$ & & & & & & & \\
\hline & & & +0.12 & $100^{\circ} \cdot 110 ?$ & & & & & & & \\
\hline & & & -0.05 & $154^{\circ} \cdot 175$ & $\mathrm{~K}$ & & $(0.80)$ & & & & \\
\hline$M$ & 4276.814 & 23375.34 & -0.06 & $94^{\circ}-63$ & $M$ & 5 & & 1.578 & 1.131 & 0.949 & \\
\hline$K$ & 4274.326 & 23388.94 & +0.03 & $100^{\circ} \cdot 150$ & $\mathrm{~K}$ & & $(0)$ & 1.46 & & & \\
\hline \multirow[t]{2}{*}{$M$} & 4274.032 & 23390.53 & 0.00 & $153^{\circ} \cdot 172$ & $M$ & 6 & $(0.464)$ & 1.227 & 1.266 & 1.180 & \\
\hline & & & -0.02 & $133^{\circ}-106$ & & & & & & & \\
\hline$M$ & 4273.363 & 23394.21 & -0.03 & $94^{\circ} \cdot 141$ & $M$ & 5 & $(0.043)$ & 1.537 & 1.140 & 1.229 & 0.38 \\
\hline$K$ & 4272.39 & 23399.5 & 0.0 & $56^{\circ}-70$ & & & & & & & \\
\hline \multirow[t]{2}{*}{$M$} & 4271.103 & 23406.58 & -0.05 & $4^{\circ}-14$ & & & & & & & \\
\hline & & & -0.13 & $55^{\circ}-106 ?$ & & & & & & & \\
\hline$K$ & 4270.329 & 23410.83 & +0.02 & $107^{\circ}-73$ & $K$ & & $(0.14)$ & 2.14 & & & 0.40 \\
\hline $\mathrm{K}$ & 4267.716 & 23425.17 & -0.14 & $58^{\circ}-115 ?$ & $K$ & & $(0)$ & 0.83 & & & \\
\hline
\end{tabular}


Table 6 (continued)

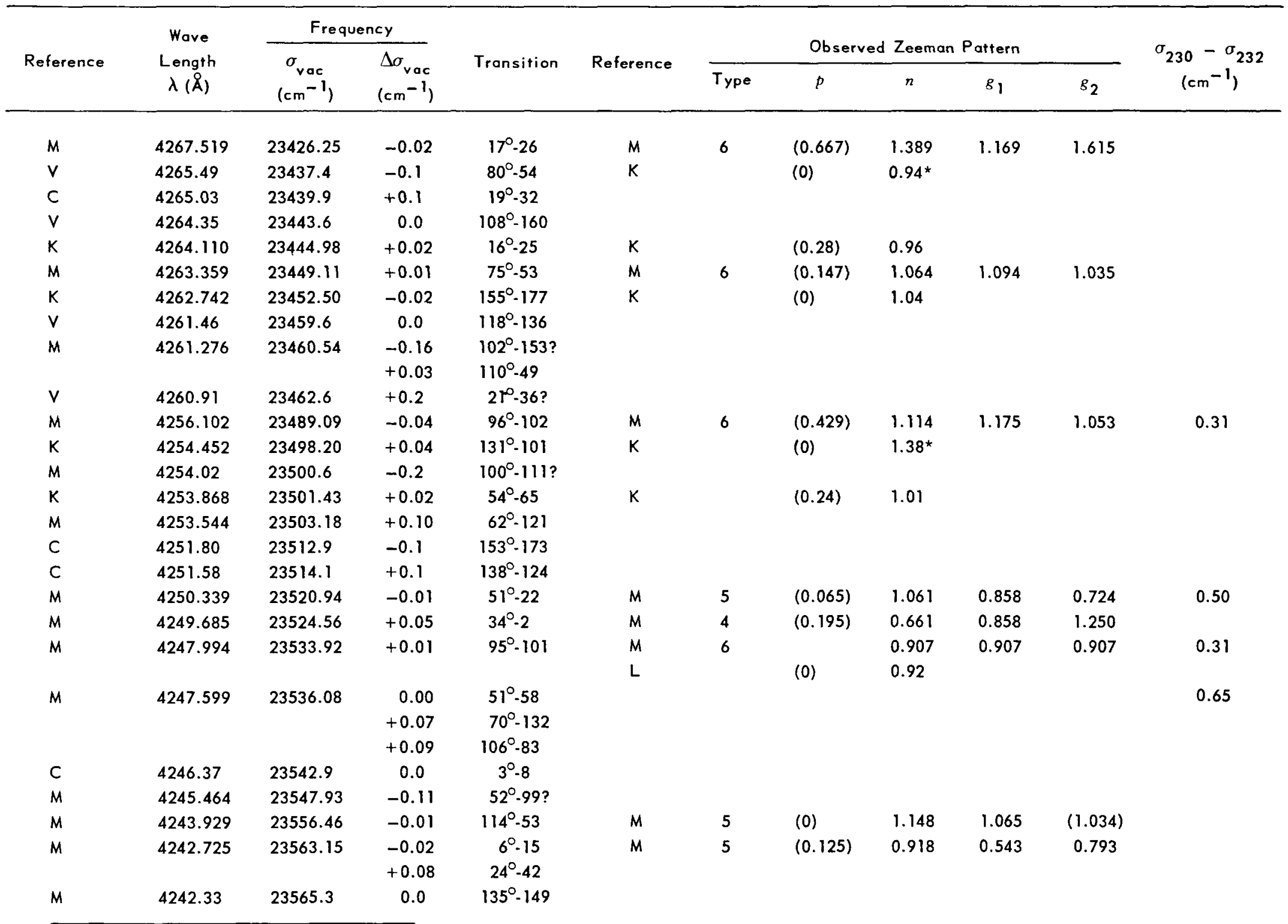

*These data are inconsistent with the classifications listed. 
Table 6 (continued)

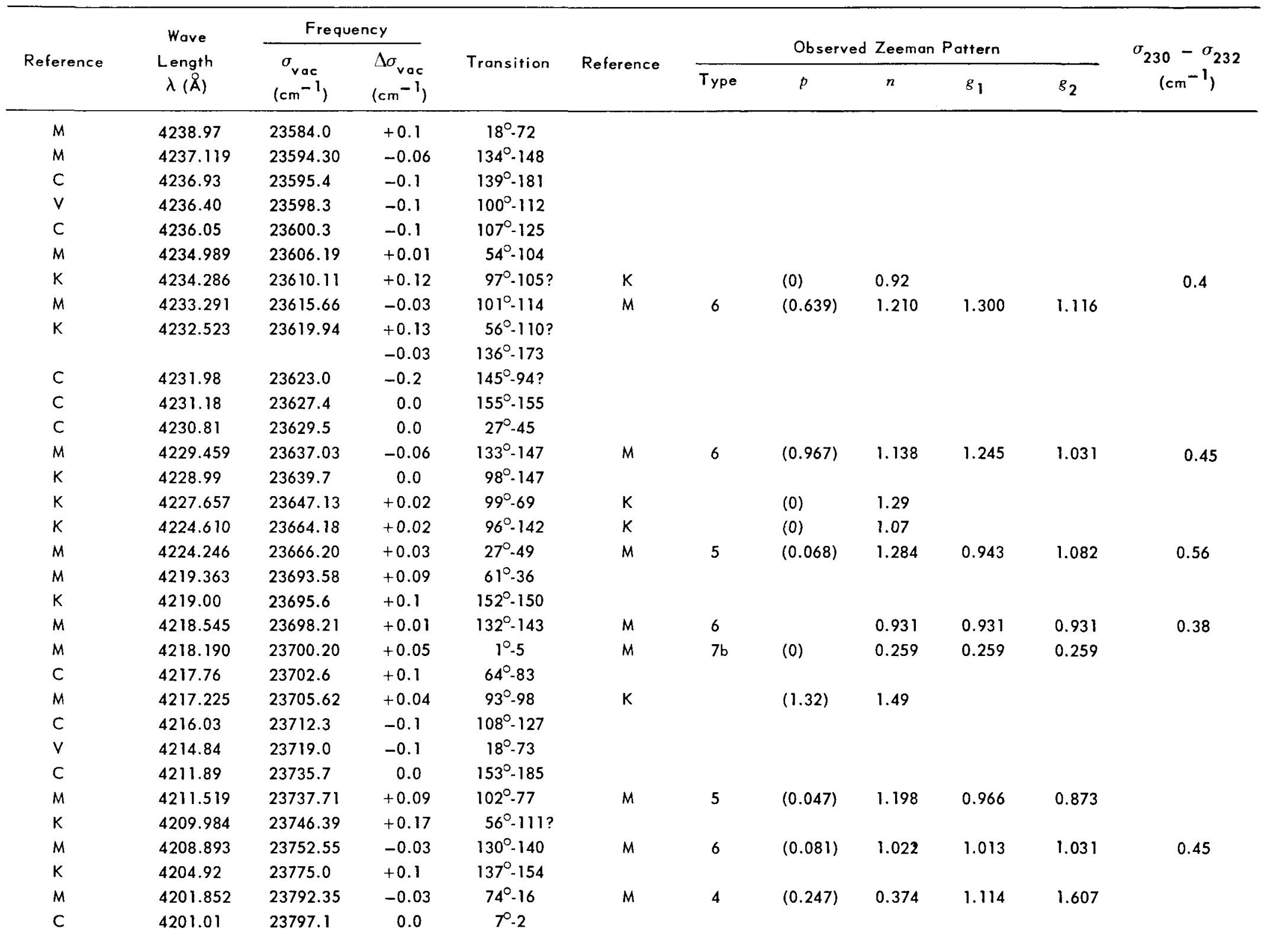




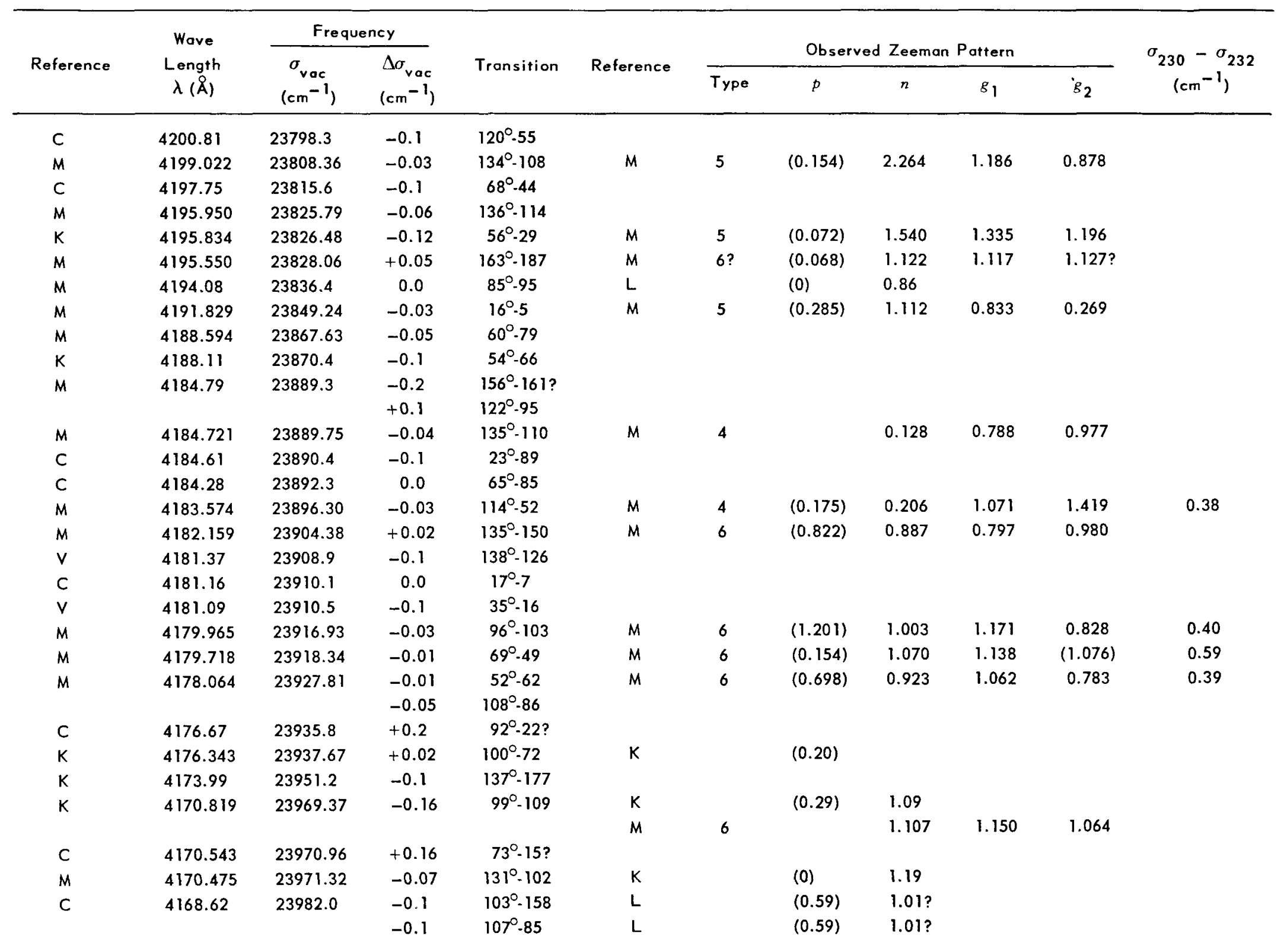


Table 6 (continued)

\begin{tabular}{|c|c|c|c|c|c|c|c|c|c|c|c|}
\hline \multirow{3}{*}{ Reference } & \multirow{3}{*}{$\begin{array}{c}\text { Wave } \\
\text { Length } \\
\lambda(\AA)\end{array}$} & \multicolumn{2}{|c|}{ Frequency } & \multirow{3}{*}{ Transition } & \multirow{3}{*}{ Reference } & \multirow{2}{*}{\multicolumn{5}{|c|}{ Observed Zeeman Pattern }} & \multirow{3}{*}{$\begin{array}{c}\sigma_{230}-\sigma_{232} \\
\left(\mathrm{~cm}^{-1}\right)\end{array}$} \\
\hline & & $\sigma_{\text {vac }}$ & $\Delta \sigma_{\text {vac }}$ & & & & & & & & \\
\hline & & $\left(\mathrm{cm}^{-1}\right)$ & $\left(\mathrm{cm}^{-1}\right)$ & & & Type & $p$ & $n$ & $g_{1}$ & $g_{2}$ & \\
\hline$M$ & 4168.574 & 23982.28 & 0.00 & $132^{\circ}-144$ & $M$ & 6 & $(0.627)$ & 0.999 & 0.931 & 1.071 & \\
\hline V & 4167.29 & 23989.7 & 0.0 & $19^{\circ}-75$ & & & & & & & \\
\hline C & 4165.75 & 23998.5 & 0.0 & $62^{\circ} \cdot 122$ & & & & & & & \\
\hline M & 4165.087 & 24002.33 & -0.07 & $153^{\circ}-174$ & & & & & & & \\
\hline \multirow[t]{2}{*}{$M$} & 4164.253 & 24007.17 & +0.03 & $95^{\circ}-102$ & $M$ & 6 & $(0.482)$ & & $(0.911)$ & 1.018 & \\
\hline & & & & & $\mathrm{K}$ & & $(0.43)$ & 0.97 & & & \\
\hline $\mathrm{K}$ & 4163.737 & 24010.14 & -0.04 & $154^{\circ}-176$ & K & & $(0.83)$ & & & & \\
\hline$M$ & 4163.653 & 24010.63 & -0.01 & $96^{\circ}-64$ & $M$ & 5 & $(0.071)$ & 1.527 & 1.174 & 1.031 & \\
\hline $\mathrm{K}$ & 4163.194 & 24013.27 & +0.02 & $116^{\circ}-94$ & $K$ & & $(0)$ & 1.11 & & & \\
\hline$M$ & 4162.685 & 24016.21 & +0.01 & $135^{\circ}-111$ & $M$ & 4 & $(0.076)$ & 0.246 & 0.787 & 0.941 & \\
\hline$M$ & 4161.93 & 24020.6 & -0.1 & $109^{\circ} \cdot 129$ & & & & & & & \\
\hline$M$ & 4161.575 & 24022.59 & +0.05 & $99^{\circ} \cdot 149$ & & & & & & & \\
\hline$K$ & 4159.658 & 24033.69 & -0.04 & $152^{\circ}-184$ & $L$ & & $(0)$ & 1.17 & & & 0.38 \\
\hline$M$ & 4156.523 & 24051.81 & -0.04 & $53^{\circ}-103$ & $M$ & 5 & $(0.115)$ & 1.400 & 0.603 & 0.831 & 0.41 \\
\hline$M$ & 4156.243 & 24053.43 & -0.01 & $54^{\circ} \cdot 105$ & & & & & & & 0.42 \\
\hline$C$ & 4153.71 & 24068.1 & -0.1 & $66^{\circ}-87$ & & & & & & & \\
\hline$c$ & 4152.17 & 24077.0 & 0.0 & $139^{\circ}-182$ & & & & & & & \\
\hline$M$ & 4151.45 & 24081.2 & 0.0 & $151^{\circ}-143$ & & & & & & & \\
\hline$c$ & 4150.35 & 24087.6 & +0.1 & $144^{\circ} \cdot 162$ & & & & & & & \\
\hline$M$ & 4149.991 & 24089.67 & 0.00 & $132^{\circ}-167$ & $M$ & 5 & $(0.035)$ & 1.293 & 0.940 & 1.005 & 0.33 \\
\hline$k$ & 4148.68 & 24097.3 & 0.0 & $71^{\circ} \cdot 91$ & & & & & & & \\
\hline$M$ & 4148.348 & 24099.18 & -0.05 & $101^{\circ}-151$ & & & & & & & \\
\hline$M$ & 4148.182 & 24100.17 & +0.01 & $35^{\circ}-2$ & $M$ & 4 & $(0.181)$ & 0.708 & 0.890 & 1.252 & \\
\hline$C$ & 4146.64 & 24109.1 & -0.1 & $5^{\circ} \cdot 47$ & & & & & & & \\
\hline C & 4144.31 & 24122.7 & -0.1 & $103^{\circ}-81$ & & & & & & & \\
\hline$K$ & 4143.617 & 24126.73 & +0.01 & $99^{\circ}-70$ & & & & & & & \\
\hline$M$ & 4142.703 & 24132.05 & 0.00 & $25^{\circ} \cdot 13$ & $M$ & 6 & $(1.181)$ & 1.039 & 1.431 & 0.643 & 0.67 \\
\hline$C$ & 4141.93 & 24136.6 & +0.1 & $138^{\circ}-160$ & & & & & & & \\
\hline$M$ & 4140.392 & 24145.52 & -0.01 & $53^{\circ}-64$ & $M$ & 6 & $(1.074)$ & 0.823 & 0.607 & 1.036 & \\
\hline$M$ & 4140.239 & 24146.38 & -0.04 & $131^{\circ} \cdot 142$ & $M$ & 6 & $(0.209)$ & 1.047 & $(1.086)$ & 1.132 & \\
\hline
\end{tabular}


Table 6 (continued)

\begin{tabular}{|c|c|c|c|c|c|c|c|c|c|c|c|}
\hline \multirow{3}{*}{ Reference } & \multirow{3}{*}{$\begin{array}{c}\text { Wave } \\
\text { Length } \\
\lambda(\AA)\end{array}$} & \multicolumn{2}{|c|}{ Frequency } & \multirow{3}{*}{ Transition } & \multirow{3}{*}{ Reference } & \multirow{2}{*}{\multicolumn{5}{|c|}{ Observed Zeeman Pottern }} & \multirow{3}{*}{$\begin{array}{c}\sigma_{230}-\sigma_{232} \\
\left(\mathrm{~cm}^{-1}\right)\end{array}$} \\
\hline & & \multirow{2}{*}{$\begin{array}{c}\sigma_{v a c} \\
\left(\mathrm{~cm}^{-1}\right)\end{array}$} & \multirow{2}{*}{$\begin{array}{l}\Delta \sigma_{v o c} \\
\left(\mathrm{~cm}^{-1}\right)\end{array}$} & & & & & & & & \\
\hline & & & & & & Type & $p$ & $n$ & $g_{1}$ & $g_{2}$ & \\
\hline $\mathrm{K}$ & 4138.87 & 24154.4 & -0.1 & $132^{\circ} \cdot 105$ & & & & & & & \\
\hline K & 4138.82 & 24154.7 & 0.0 & $36^{\circ}-52$ & & & & & & & \\
\hline$M$ & 4136.383 & 24168.89 & -0.04 & $162^{\circ} \cdot 183$ & $M$ & 6 & $(0.954)$ & 1.145 & 1.219 & 1.071 & \\
\hline$c$ & 4135.01 & 24176.9 & -0.1 & $22^{\circ}-82$ & & & & & & & \\
\hline $\mathrm{K}$ & 4134.110 & 24182.20 & +0.03 & $95^{\circ}-142$ & $M$ & 5 & $(0.111)$ & 1.937 & 0.938 & 1.160 & \\
\hline \multirow[t]{2}{*}{$M$} & 413.1 .433 & 24197.88 & -0.04 & $94^{\circ}-100$ & $M$ & 6 & $(0.367)$ & & 1.133 & 1.026 & \\
\hline & & & & & K & & $(0.35)$ & 1.04 & & & \\
\hline M & 4130.68 & 24202.3 & -0.1 & $153^{\circ} \cdot 151$ & & & & & & & \\
\hline C & 4129.92 & 24206.7 & 0.0 & $20^{\circ} .79$ & & & & & & & \\
\hline$c$ & 4127.38 & 24221.6 & -0.1 & $108^{\circ} \cdot 161$ & & & & & & & \\
\hline$M$ & 4124.658 & 24237.59 & -0.01 & $19^{\circ} .9$ & $L$ & & $(0)$ & 1.13 & & & \\
\hline$M$ & 4123.569 & 24244.01 & -0.18 & $75^{\circ}-51 ?$ & & & & & & & \\
\hline$C$ & 4121.86 & 24254.1 & 0.0 & $103^{\circ}-82$ & & & & & & & \\
\hline $\mathrm{K}$ & 4120.03 & 24264.9 & -0.1 & $164^{\circ}-180$ & $\mathrm{~K}$ & & (0) & 1.29 & & & \\
\hline V & 4118.55 & 24273.6 & 0.0 & $106^{\circ}-160$ & & & & & & & \\
\hline$c$ & 4118.25 & 24275.3 & -0.1 & $59^{\circ} .116$ & & & & & & & \\
\hline K & 4117.86 & 24277.6 & +0.1 & $100^{\circ}-114$ & & & & & & & \\
\hline$M$ & 4116.718 & 24284.37 & -0.02 & $93^{\circ}-140$ & $M$ & 5 & $(0.158)$ & 2.147 & 0.718 & 1.035 & 0.35 \\
\hline$c$ & 4116.32 & 24286.7 & 0.0 & $99^{\circ}-71$ & & & & & & & \\
\hline $\mathrm{K}$ & 4112.326 & 24310.30 & -0.01 & $163^{\circ}-179$ & $K$ & & $(0)$ & 1.43 & & & \\
\hline$c$ & 4111.84 & 24313.2 & +0.1 & $65^{\circ}-86$ & & & & & & & \\
\hline$C$ & 4111.43 & 24315.6 & +0.1 & $92^{\circ}-21$ & & & & & & & \\
\hline$M$ & 4110.870 & 24318.89 & -0.01 & $134^{\circ}-109$ & $K$ & & $(0)$ & 1.50 & & & \\
\hline$M$ & 4110.640 & 24320.28 & -0.01 & $6^{\circ}-1$ & $M$ & 6 & $(0.777)$ & 1.333 & 0.556 & 2.110 & \\
\hline \multirow[t]{2}{*}{$M, K$} & 4110.525 & 24320.95 & -0.16 & $146^{\circ}-135 ?$ & & & & & & & \\
\hline & & & 0.00 & $133^{\circ}-107$ & & & & & & & \\
\hline K & 4110.09 & 24323.5 & -0.1 & $98^{\circ}-107$ & & & & & & & \\
\hline$M$ & 4109.395 & 24327.64 & +0.04 & $76^{\circ}-51$ & $M$ & 6 & $(0.317)$ & & 1.113 & $(0.986)$ & \\
\hline$K$ & 4106.130 & 24346.98 & -0.05 & $99^{\circ} .110$ & & & & & & & \\
\hline$K$ & 4105.924 & 24348.21 & -0.02 & $54^{\circ} \cdot 25$ & $K$ & & (0) & 1.12 & & & \\
\hline
\end{tabular}


Table 6 (cont inued)

\begin{tabular}{|c|c|c|c|c|c|c|c|c|c|c|c|}
\hline \multirow{3}{*}{ Reference } & \multirow{3}{*}{$\begin{array}{c}\text { Wave } \\
\text { Length } \\
\lambda(\AA)\end{array}$} & \multicolumn{2}{|c|}{ Frequency } & \multirow{3}{*}{ Transition } & \multirow{3}{*}{ Reference } & \multirow{2}{*}{\multicolumn{5}{|c|}{ Observed Zeeman Pattern }} & \multirow{3}{*}{$\begin{array}{c}\sigma_{230}-\sigma_{232} \\
\left(\mathrm{~cm}^{-1}\right)\end{array}$} \\
\hline & & \multirow{2}{*}{$\begin{array}{c}\sigma_{\mathrm{vac}} \\
\left(\mathrm{cm}^{-1}\right)\end{array}$} & \multirow{2}{*}{$\begin{array}{l}\Delta \sigma_{v a c} \\
\left(\mathrm{~cm}^{-1}\right)\end{array}$} & & & & & & & & \\
\hline & & & & & & Type & $p$ & $n$ & $g_{1}$ & $g_{2}$ & \\
\hline \multirow[t]{2}{*}{ M } & 4105.343 & 24351.65 & -0.05 & $34^{\circ}-15$ & M & 6 & $(0.085)$ & 0.833 & 0.865 & 0.801 & \\
\hline & & & +0.09 & $114^{\circ} \cdot 51$ & $K$ & & $(0)$ & 1.10 & & & \\
\hline$M$ & 4104.392 & 24357.30 & -0.05 & $94^{\circ} \cdot 101$ & $M$ & 6 & $(0.791)$ & 1.017 & 1.130 & 0.904 & 0.62 \\
\hline \multirow[t]{2}{*}{$M$} & 4103.67 & 24361.6 & 0.0 & $40^{\circ}-18$ & & & & & & & \\
\hline & & & 0.0 & $99^{\circ} \cdot 150$ & & & & & & & \\
\hline K & 4101.337 & 24375.44 & -0.03 & $155^{\circ} \cdot 178$ & $\mathrm{~K}$ & & $(0.25)$ & 1.11 & & & \\
\hline \multirow[t]{2}{*}{$\mathrm{K}$} & 4100.838 & 24378.40 & -0.07 & $58^{\circ}-116$ & $K, L$ & & $(0)$ & $1.09 ?$ & & & \\
\hline & & & -0.10 & $152^{\circ}-173$ & $K, L$ & & $(0)$ & $1.09 ?$ & & & \\
\hline$M$ & 4098.939 & 24389.70 & +0.05 & $75^{\circ} \cdot 17$ & $M$ & 5 & $(0.062)$ & 1.282 & 1.096 & 0.972 & \\
\hline$M$ & 4097.686 & 24397.13 & +0.04 & $121^{\circ}-55$ & & & & & & & \\
\hline$M$ & 4097.339 & 24399.20 & -0.02 & $131^{\circ} \cdot 103$ & & & & & & & \\
\hline $\mathrm{C}$ & 4096.33 & 24405.2 & 0.0 & $138^{\circ}-127$ & & & & & & & \\
\hline$M$ & 1094.751 & 24414.64 & +0.04 & $26^{\circ}-13$ & $M$ & 6 & $(0.700)$ & 0.866 & 1.103 & 0.635 & 0.47 \\
\hline$K$ & 4094.51 & 24416.1 & 0.0 & $137^{\circ}-118$ & & & & & & & \\
\hline$M$ & 4093.394 & 24422.74 & 0.00 & $52^{\circ}-63$ & $M$ & 6 & $(0.277)$ & 1.020 & 1.076 & 0.965 & 0.38 \\
\hline \multirow[t]{2}{*}{$K$} & 4092.32 & 24429.1 & 0.0 & $155^{\circ}-157$ & & & & & & & \\
\hline & & & +0.1 & $97^{\circ} .146$ & & & & & & & \\
\hline 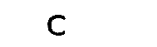 & 4091.75 & 24432.6 & 0.0 & $63^{\circ} \cdot 84$ & & & & & & & \\
\hline$M$ & 4091.53 & 24433.9 & 0.0 & $53^{\circ}-24$ & & & & & & & \\
\hline $\mathrm{K}$ & 4091.341 & 24434.99 & +0.02 & $95^{\circ} \cdot 103$ & $K$ & & $(0.27)$ & 0.92 & & & \\
\hline v & 4087.21 & 24459.7 & +0.1 & $104^{\circ}-83$ & & & & & & & \\
\hline$M$ & 4086.520 & 24463.82 & -0.04 & $67^{\circ}-13$ & $M$ & 5 & $(0.377)$ & 2.532 & 1.393 & 0.637 & 0.71 \\
\hline$K$ & 4085.810 & 24468.04 & 0.00 & $80^{\circ}-18$ & $\mathrm{~K}$ & & $(0)$ & 1.17 & & & \\
\hline$M$ & 4085.036 & 24472.70 & +0.04 & $151^{\circ}-167$ & $M$ & 6 & $(0.704)$ & 1.063 & 1.127 & 0.998 & 0.42 \\
\hline$K$ & 4083.390 & 24482.57 & 0.00 & $154^{\circ}-154$ & & & & & & & \\
\hline \multirow[t]{2}{*}{$M$} & 4082.263 & 24489.33 & 0.00 & $16^{\circ}-68$ & $M$ & 5 & & 0.953 & $(0.832)$ & 0.880 & 0.37 \\
\hline & & & & & $L$ & & $(0)$ & 0.94 & & & \\
\hline$V$ & 4081.82 & 24492.0 & +0.1 & $102^{\circ} \cdot 155$ & & & & & & & \\
\hline$M$ & 4079.91 & 24503.5 & 0.0 & $64^{\circ}-85$ & & & & & & & \\
\hline$M, C$ & 4079.606 & 24505.27 & -0.02 & $19^{\circ} .76$ & & & & & & & \\
\hline C & 4078.46 & 24512.2 & +0.2 & $19^{\circ}-33 ?$ & & & & & & & \\
\hline$c$ & 4076.63 & 24523.2 & 0.0 & $56^{\circ}-114$ & & & & & & & \\
\hline $\mathrm{K}$ & 4075.719 & 24528.65 & 0.00 & $95^{\circ} .64$ & $K$ & & (0) & 0.66 & & & \\
\hline
\end{tabular}


Table 6 (cont inued)

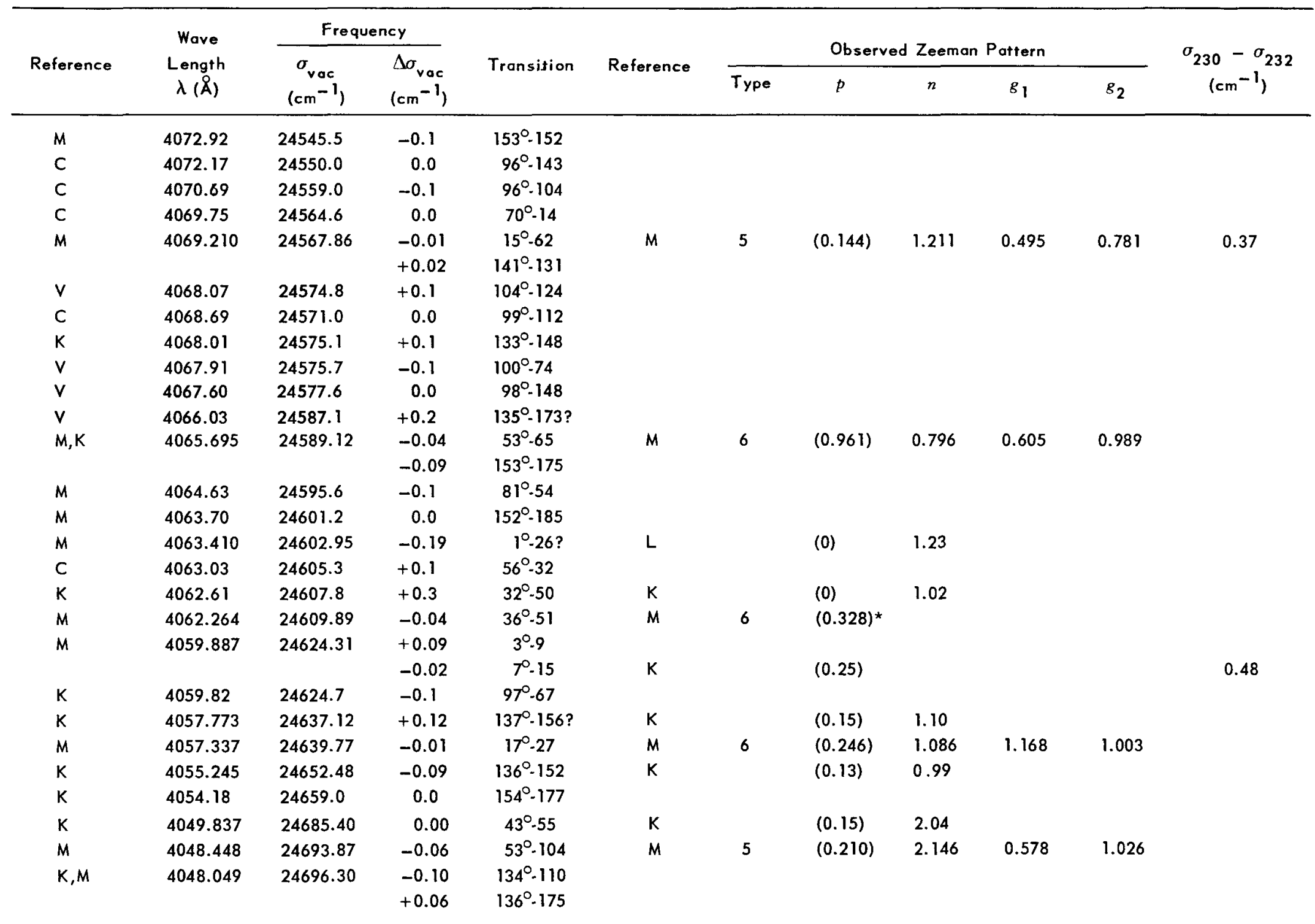

*These data are inconsistent with the classifications listed. 
Table 6 (cont inued)

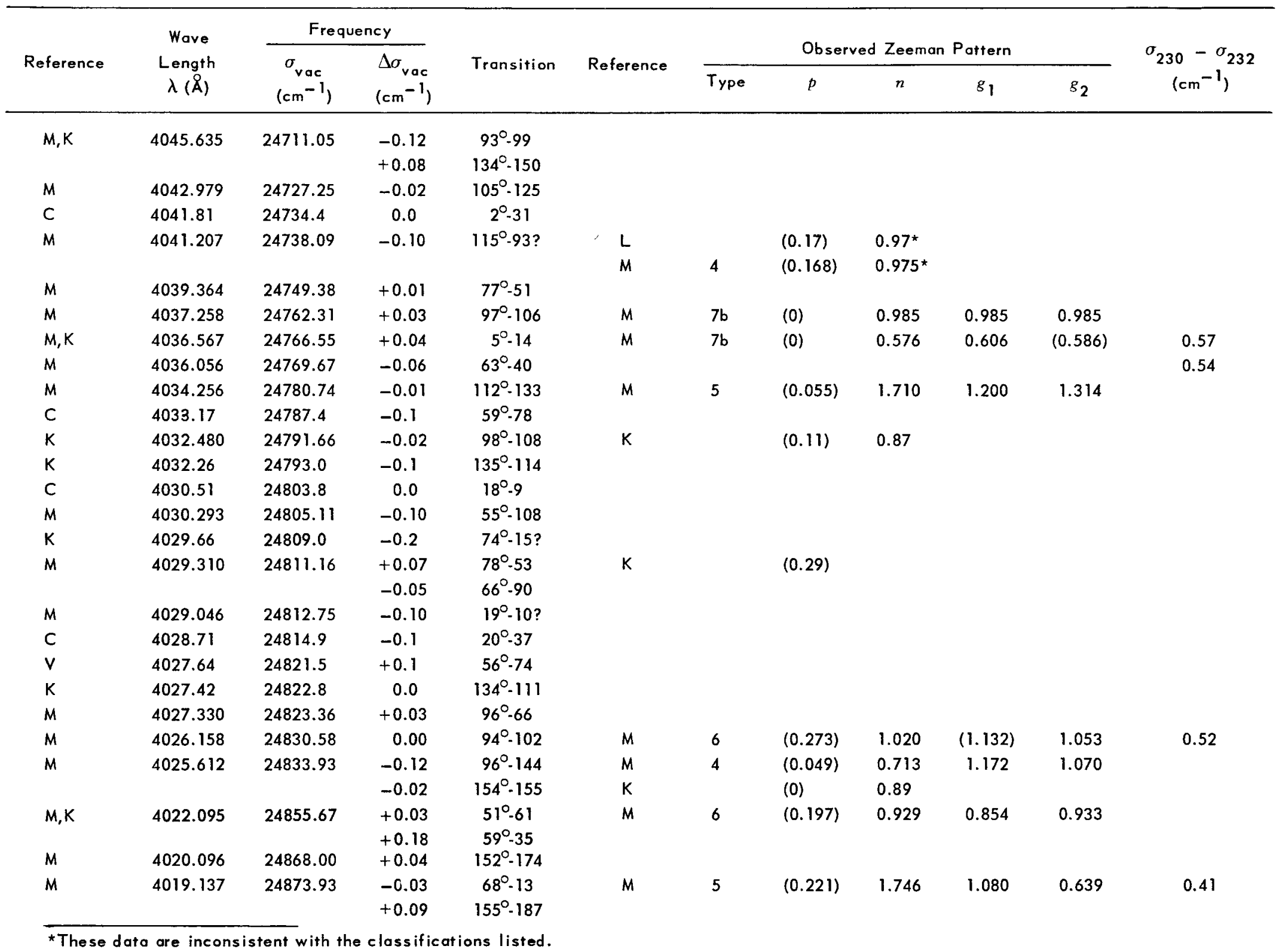


Table 6 (cont inued)

\begin{tabular}{|c|c|c|c|c|c|c|c|c|c|c|c|}
\hline \multirow{3}{*}{ Reference } & \multirow{3}{*}{$\begin{array}{c}\text { Wave } \\
\text { Length } \\
\lambda(\AA)\end{array}$} & \multicolumn{2}{|c|}{ Frequency } & \multirow{3}{*}{ Transition } & \multirow{3}{*}{ Reference } & \multirow{2}{*}{\multicolumn{5}{|c|}{ Observed Zeeman Pattern }} & \multirow{3}{*}{$\begin{array}{c}\sigma_{230}-\sigma_{232} \\
\left(\mathrm{~cm}^{-1}\right)\end{array}$} \\
\hline & & $\sigma_{\text {vac }}$ & $\Delta \sigma_{\text {vac }}$ & & & & & & & & \\
\hline & & $\left(\mathrm{cm}^{-1}\right)$ & $\left(\mathrm{cm}^{-1}\right)$ & & & Type & $p$ & $n$ & $g_{1}$ & $s_{2}$ & \\
\hline c & 4018.37 & 24878.7 & -0.1 & $104^{\circ}-125$ & & & & & & & \\
\hline M & 4017.495 & 24884.10 & -0.01 & $132^{\circ}-145$ & & & & & & & \\
\hline C & 4016.47 & 24890.5 & -0.1 & $58^{\circ} .78$ & & & & & & & \\
\hline$k$ & 4016.310 & 24891.47 & -0.01 & $60^{\circ}-80$ & K & & $(0.93)$ & 1.32 & & & \\
\hline M & 4015.117 & 24898.84 & +0.04 & $108^{\circ}-87$ & & & & & & & \\
\hline M & 4014.533 & 24902.48 & -0.14 & $70^{\circ}-49$ & $\mathrm{~K}$ & & $(0.24)$ & 1.07 & & & \\
\hline \multirow[t]{2}{*}{ M } & 4013.264 & 24910.33 & -0.04 & $10^{\circ}-18$ & K & & $(0.23)$ & 1.19 & & & \\
\hline & & & -0.01 & $99^{\circ}-72$ & & & & & & & \\
\hline M & 4012.497 & 24915.12 & -0.14 & $76^{\circ}-16 ?$ & & & & & & & \\
\hline M & 4011.772 & 24919.62 & -0.02 & $17^{0}-28$ & K & & (0) & 1.16 & & & \\
\hline \multirow[t]{2}{*}{$v$} & 4011.62 & 24920.6 & +0.1 & $108^{\circ}-88$ & & & & & & & \\
\hline & & & +0.1 & $134^{\circ}-112$ & & & & & & & \\
\hline M & 4011.026 & 24923.24 & -0.07 & $64^{\circ} .86$ & & & & & & & \\
\hline M & 4010.50 & 24927.5 & +0.2 & $35^{\circ}-15 ?$ & & & & & & & \\
\hline k & 4010.426 & 24927.99 & +0.06 & $137^{\circ}-157$ & & & & & & & \\
\hline M & 4009.546 & 24933.43 & +0.01 & $19^{\circ} .77$ & & & & & & & \\
\hline$M$ & 4007.027 & 24949.13 & -0.02 & $97^{\circ}-68$ & M & 5 & $(0.042)$ & 1.218 & 0.991 & 0.901 & 0.37 \\
\hline K & 4006.386 & 24953.12 & +0.02 & $132^{\circ}-168$ & $\mathrm{~K}$ & & $(0)$ & 1.27 & & & 0.41 \\
\hline \multirow[t]{2}{*}{ M } & 4005.549 & 24958.34 & +0.12 & $53^{\circ}-66 ?$ & & & & & & & \\
\hline & & & -0.05 & $101^{\circ}-116$ & L & & $(0.66)$ & 1.08 & & & \\
\hline M & 4003.316 & 24972.26 & -0.02 & $95^{\circ} .65$ & M & 4 & & 0.745 & $(0.911)$ & 0.976 & \\
\hline K & 4003.110 & 24973.54 & -0.01 & $132^{\circ}-146$ & $k$ & & $(0.45)$ & 0.84 & & & 0.43 \\
\hline c & 4002.37 & 24978.2 & 0.0 & $110^{\circ} .132$ & & & & & & & \\
\hline M & 4001.740 & 24982.09 & -0.01 & $72^{\circ}-50$ & K & & $(0.36)$ & 1.04 & & & \\
\hline$M$ & 3999.95 & 24993.3 & 0.0 & $55^{\circ}-69$ & & & & & & & \\
\hline$\checkmark$ & 3998.98 & 24999.3 & +0.1 & $146^{\circ}-94$ & & & & & & & \\
\hline$M$ & 3997.864 & 25006.31 & +0.01 & $96^{\circ}-105$ & M & 6 & $(0.785)$ & 1.060 & 1.178 & 0.952 & 0.43 \\
\hline$K$ & 3997.468 & 25008.79 & -0.03 & $97^{\circ}-147$ & K & & $(0)$ & 1.12 & & & \\
\hline \multirow[t]{2}{*}{ K } & 3996.062 & 25017.59 & +0.07 & $153^{\circ}-153$ & $M$ & 5 & $(0.042)$ & & 1.260 & (1.176) & 0.37 \\
\hline & & & & & $L$ & & $(0)$ & 1.64 & & & \\
\hline M & 3993.725 & 25032.20 & -0.05 & $131^{\circ}-143$ & & & & & & & \\
\hline M & 3992.278 & 25041.27 & -0.03 & $131^{\circ} \cdot 104$ & & & & & & & \\
\hline K & 3992.05 & 25042.7 & 0.0 & $17^{\circ}-70$ & & & & & & & \\
\hline
\end{tabular}


Table 6 (continued)

\begin{tabular}{|c|c|c|c|c|c|c|c|c|c|c|c|}
\hline \multirow{3}{*}{ Reference } & \multirow{3}{*}{$\begin{array}{c}\text { Wave } \\
\text { Length } \\
\lambda(\AA)\end{array}$} & \multicolumn{2}{|c|}{ Frequency } & \multirow{3}{*}{ Transition } & \multirow{3}{*}{ Reference } & \multirow{2}{*}{\multicolumn{5}{|c|}{ Observed Zeeman Pattern }} & \multirow{3}{*}{$\begin{array}{c}\sigma_{230}-\sigma_{232} \\
\left(\mathrm{~cm}^{-1}\right)\end{array}$} \\
\hline & & & & & & & & & & & \\
\hline & & $\begin{array}{c}\text { vac } \\
\left(\mathrm{cm}^{-1}\right)\end{array}$ & $\left(\mathrm{cm}^{-1}\right)$ & & & Type & $p$ & $n$ & $g_{1}$ & $g_{2}$ & \\
\hline$M$ & 3991.607 & 25045.48 & -0.02 & $99^{\circ}-73$ & & & & & & & \\
\hline C & 3991.07 & 25048.9 & +0.1 & $2^{\circ}-32$ & & & & & & & \\
\hline \multirow[t]{2}{*}{$K$} & 3988.845 & 25062.85 & +0.04 & $15^{\circ}-63$ & & & & & & & \\
\hline & & & & & $L$ & & $(0.23)$ & 1.64 & & & \\
\hline$K$ & 3988.02 & 25068.0 & 0.0 & $95^{\circ}-143$ & $\mathbf{L}$ & & $(0)$ & 1.00 & & & \\
\hline \multirow[t]{2}{*}{$M$} & 3988.015 & 25068.07 & -0.13 & $54^{\circ}-67$ & K & & $(0.30)$ & 1.05 & & & 0.49 \\
\hline & & & +0.15 & $152^{\circ}-151$ & & & & & & & \\
\hline$M$ & 3987.713 & 25069.94 & -0.08 & $55^{\circ}-27$ & & & & & & & \\
\hline$M$ & 3987.226 & 25073.00 & +0.10 & $130^{\circ} \cdot 141$ & & & & & & & \\
\hline$M$ & 3987.07 & 25074.0 & 0.0 & $133^{\circ} \cdot 170$ & & & & & & & \\
\hline$M$ & 3986.637 & 25076.71 & -0.01 & $135^{\circ}-174$ & & & & & & & \\
\hline C & 3986.43 & 25078.0 & -0.1 & $18^{\circ}-33 ?$ & & & & & & & \\
\hline \multirow[t]{2}{*}{$M$} & 3982.236 & 25104.42 & +0.02 & $57^{\circ}-34$ & & & & & & & \\
\hline & & & +0.01 & $100^{\circ}-152$ & & & & & & & \\
\hline$K$ & 3982.105 & 25105.27 & 0.00 & $30^{\circ}-14$ & $K$ & & $(1.16)$ & 0.97 & & & \\
\hline$M$ & 3981.539 & 25108.84 & +0.02 & $34^{\circ}-1$ & & & & & & & \\
\hline$M$ & 3981.110 & 251.52 & -0.07 & $162^{\circ}-184$ & $M$ & 6 & $(0.792)$ & 1.168 & 1.229 & 1.107 & 0.50 \\
\hline$K$ & 3979.047 & 25124.57 & +0.02 & $136^{\circ}-153$ & $M$ & 6 & $(0.832)$ & 1.053 & 0.961 & 1.145 & \\
\hline$M$ & 3976.418 & 25141.18 & -0.01 & $53^{\circ}-105$ & $M$ & 5 & $(0.175)$ & 1.823 & 0.605 & 0.953 & 0.38 \\
\hline $\mathrm{C}$ & 3975.85 & 25144.8 & +0.1 & $107^{\circ}-128$ & & & & & & & \\
\hline$K$ & 3974.227 & 25155.04 & +0.04 & $56^{\circ} .75$ & $K$ & & $(0.63)$ & 1.21 & & & \\
\hline$M$ & 3969.834 & 25182.85 & +0.10 & $135^{\circ}-115 ?$ & & & & & & & \\
\hline$M, K$ & 3969.532 & 25184.79 & +0.02 & $155^{\circ}-158$ & $K$ & & $(0)$ & 0.97 & & & \\
\hline$M$ & 3969.343 & 25185.97 & +0.07 & $109^{\circ}-130$ & & & & & & & \\
\hline$M$ & 3969.003 & 25188.14 & +0.06 & $27^{\circ}-13$ & & & & & & & 0.65 \\
\hline$M$ & 3967.214 & 25199.50 & +0.03 & $3^{\circ}-10$ & & & & & & & 0.51 \\
\hline$K$ & 3966.72 & 25202.6 & -0.1 & $17^{\circ}-71$ & & & & & & & \\
\hline$K$ & 3966.24 & 25205.7 & 0.0 & $54^{\circ}-106$ & & & & & & & \\
\hline$M$ & 3963.226 & 25224.83 & -0.05 & $153^{\circ}-176$ & $M$ & 6 & $(0.706)$ & 1.201 & 1.266 & 1.136 & 0.43 \\
\hline$c$ & 3962.93 & 25226.7 & -0.1 & $138^{\circ} \cdot 182$ & & & & & & & \\
\hline
\end{tabular}


Table 6 (continued)

\begin{tabular}{|c|c|c|c|c|c|c|c|c|c|c|c|}
\hline \multirow{3}{*}{ Reference } & \multirow{3}{*}{$\begin{array}{c}\text { Wove } \\
\text { Length } \\
\lambda(\AA)\end{array}$} & \multicolumn{2}{|c|}{ Frequency } & \multirow{3}{*}{ Transition } & \multirow{3}{*}{ Reference } & \multirow{2}{*}{\multicolumn{5}{|c|}{ Observed Zeeman Pattern }} & \multirow{3}{*}{$\begin{array}{c}\sigma_{230}-\sigma_{232} \\
\left(\mathrm{~cm}^{-1}\right)\end{array}$} \\
\hline & & \multirow{2}{*}{$\begin{array}{c}\sigma_{\text {vac }} \\
\left(\mathrm{cm}^{-1}\right)\end{array}$} & \multirow{2}{*}{$\begin{array}{c}\Delta \sigma_{\mathrm{vac}} \\
\left(\mathrm{cm}^{-1}\right)\end{array}$} & & & & & & & & \\
\hline & & & & & & Type & $p$ & $n$ & $g_{1}$ & $g_{2}$ & \\
\hline M & 3962.346 & 25230.46 & -0.08 & $20^{\circ} \cdot 80$ & $M$ & 4 & $(0.073)$ & 0.900 & 1.272 & 1.126 & \\
\hline K & 3961.516 & 25235.75 & -0.03 & $43^{\circ}-19$ & & & & & & & \\
\hline$M$ & 3960.339 & 25243.25 & +0.03 & $82^{\circ}-54$ & $M$ & 6 & $(0.067)$ & 1.232 & 1.219 & 1.246 & \\
\hline$M$ & 3959.295 & 25249.87 & -0.11 & $161^{\circ} \cdot 164 ?$ & & & & & & & \\
\hline K & 3959.221 & 25250.38 & 0.00 & $99^{\circ}-114$ & K & & (0) & 1.13 & & & \\
\hline $\mathrm{V}$ & 3957.16 & 25263.5 & 0.0 & $61^{\circ}-122$ & & & & & & & \\
\hline$M$ & 3956.682 & 25266.58 & 0.00 & $4^{\circ}-13$ & $M$ & 4 & $(0.043)$ & 0.590 & 0.719 & 0.633 & \\
\hline$M$ & 3956.603 & 25267.08 & -0.02 & $151^{\circ}-145$ & $M$ & 5 & & 1.414 & $(1.128)$ & 1.065 & \\
\hline $\mathrm{K}$ & 3955.255 & 25275.69 & +0.12 & $81^{\circ}-94$ & $K$ & & $(0)$ & 1.09 & & & \\
\hline$K$ & 3955.109 & 25276.63 & -0.05 & $135^{\circ}-151$ & & & & & & & \\
\hline$c$ & 3953.94 & 25284.1 & 0.0 & $65^{\circ} .87$ & & & & & & & \\
\hline$M$ & 3952.451 & 25293.60 & +0.05 & $102^{\circ}-157$ & & & & & & & \\
\hline $\mathrm{K}$ & 3951.116 & 25302.17 & -0.02 & $98^{\circ}-109$ & $K$ & & $(0.57)$ & & & & \\
\hline \multirow[t]{2}{*}{$M$} & 3950.394 & 25306.79 & -0.01 & $132^{\circ}-106$ & $M$ & 4 & & 0.769 & $(0.931)$ & 0.977 & \\
\hline & & & & & $L$ & & $(0)$ & 0.79 & & & \\
\hline$M$ & 3949.006 & 25315.66 & -0.01 & $102^{\circ} \cdot 119$ & $K$ & & (0) & 0.06 & 1.09 & & \\
\hline$M$ & 3948.908 & 25316.32 & -0.01 & $131^{\circ} \cdot 144$ & $M$ & 6 & $(0.074)$ & 1.079 & 1.087 & 1.071 & \\
\hline$M$ & 3946.151 & 25333.98 & +0.03 & $162^{\circ}-172$ & $\mathrm{~L}$ & & $(0)$ & 1.45 & & & \\
\hline $\mathrm{K}$ & 3945.824 & 25336.10 & +0.01 & $151^{\circ} \cdot 168$ & $\mathrm{~K}$ & & $(0.56)$ & 1.17 & & & 0.54 \\
\hline$M$ & 3943.694 & 25349.77 & -0.11 & $55^{\circ}-28 ?$ & & & & & & & \\
\hline \multirow[t]{3}{*}{$M$} & 3943.345 & 25352.01 & -0.05 & $95^{\circ} \cdot 144$ & $M$ & 5 & $(0.081)$ & 1.603 & 0.906 & 1.061 & \\
\hline & & & -0.10 & $59^{\circ}-117 ?$ & & & & & & & \\
\hline & & & -0.08 & $94^{\circ} \cdot 64$ & & & & & & & \\
\hline $\mathrm{K}$ & 3942.69 & 25356.2 & +0.1 & $155^{\circ} \cdot 179$ & & & & & & & \\
\hline$M$ & 3942.646 & 25356.50 & -0.04 & $151^{\circ}-146$ & & & & & & & \\
\hline$K$ & 3942.044 & 25360.40 & 0.00 & $163^{\circ}-180$ & $K$ & & $(0)$ & 1.03 & & & \\
\hline$M$ & 3941.727 & 25362.41 & -0.03 & $138^{\circ} \cdot 128$ & & & & & & & \\
\hline$M$ & 3939.788 & 25374.89 & -0.07 & $153^{\circ}-186$ & & & & & & & \\
\hline$K$ & 3938.739 & 25381.68 & -0.08 & $41^{\circ} \cdot 18$ & $\mathrm{~K}$ & & $(0.43)$ & & & & \\
\hline $\mathrm{C}$ & 3938.43 & 25383.7 & 0.0 & $51^{\circ} .98$ & & & & & & & \\
\hline
\end{tabular}


Table 6 (continued)

\begin{tabular}{|c|c|c|c|c|c|c|c|c|c|c|c|}
\hline \multirow{3}{*}{ Reference } & \multirow{3}{*}{$\begin{array}{c}\text { Wave } \\
\text { Length } \\
\lambda(\AA)\end{array}$} & \multicolumn{2}{|c|}{ Frequency } & \multirow{3}{*}{ Transition } & \multirow{3}{*}{ Reference } & \multirow{2}{*}{\multicolumn{5}{|c|}{ Observed Zeemon Pattern }} & \multirow{3}{*}{$\begin{array}{c}\sigma_{230}-\sigma_{232} \\
\left(\mathrm{~cm}^{-1}\right)\end{array}$} \\
\hline & & \multirow{2}{*}{$\begin{array}{c}\sigma_{\text {vac }} \\
\left(\mathrm{cm}^{-1}\right)\end{array}$} & \multirow{2}{*}{$\begin{array}{l}\Delta \sigma_{\mathrm{vac}} \\
\left(\mathrm{cm}^{-1}\right)\end{array}$} & & & & & & & & \\
\hline & & & & & & Type & $p$ & $n$ & $g_{1}$ & $g_{2}$ & \\
\hline$M$ & 3937.888 & 25387.17 & 0.00 & $36^{\circ}-2$ & $M$ & 4 & $(0.109)$ & 0.921 & 1.032 & 1.254 & \\
\hline$M$ & 3937.043 & 25392.61 & +0.01 & $54^{\circ}-68$ & $M$ & 6 & $(0.506)$ & 0.988 & 1.089 & 0.886 & 0.39 \\
\hline \multirow[t]{2}{*}{$k$} & 3935.632 & 25401.71 & +0.09 & $86^{\circ}-95$ & $M$ & & $(0.120)$ & 0.270 & 1.126 & 0.886 & \\
\hline & & & & & & & $(0.120)$ & 0.270 & $(1.168)$ & 0.922 & \\
\hline$M$ & 3935.180 & 25404.63 & -0.06 & $52^{\circ}-101$ & $\mathrm{~K}$ & & $(0)$ & $1.21^{\star}$ & & & \\
\hline K & 3934.18 & 25411.1 & 0.0 & $152^{\circ}-152$ & & & & & & & \\
\hline$k$ & 3932.231 & 25423.68 & -0.04 & $131^{\circ} \cdot 167$ & K & & $(0)$ & 0.63 & & & 0.43 \\
\hline$M$ & 3931.99 & 25425.2 & +0.1 & $100^{\circ} .76$ & & & & & & & \\
\hline$K$ & 3930.33 & 25436.0 & 0.0 & $53^{\circ}-25$ & & & & & & & \\
\hline$M$ & 3929.671 & 25440.25 & -0.01 & $69^{\circ} \cdot 13$ & $M$ & 5 & $(0.251)$ & 1.887 & 1.138 & 0.638 & 0.70 \\
\hline$V, K$ & 3928.95 & 25444.9 & -0.1 & $24^{\circ}-45$ & & & & & & & \\
\hline$v$ & 3928.84 & 25445.6 & +0.2 & $102^{\circ}-80 ?$ & & & & & & & \\
\hline $\mathrm{K}$ & 3927.427 & 25454.78 & +0.01 & $152^{\circ}-175$ & $\mathrm{~L}$ & & $(0.19)$ & 1.10 & & & 0.36 \\
\hline$K$ & 3927.182 & 25456.37 & +0.01 & $162^{\circ}-173$ & $\mathrm{~K}$ & & (0) & 1.92 & & & 0.44 \\
\hline$M$ & 3926.711 & 25459.40 & +0.02 & $98^{\circ} \cdot 70$ & & & & & & & \\
\hline$M$ & 3925.320 & 25467.36 & +0.09 & $40^{\circ} \cdot 1$ & $\mathrm{~K}$ & & $(0.51)$ & & & & \\
\hline$M$ & 3925.216 & 25469.12 & +0.05 & $4^{\circ}-47$ & & & & & & & \\
\hline$K$ & 3925.11 & 25469.8 & 0.0 & $17^{\circ}-29$ & $\mathrm{~K}$ & & $(0)$ & 1.18 & & & \\
\hline \multirow[t]{2}{*}{$K$} & 3924.99 & 25470.6 & 0.0 & $57^{\circ}-77$ & & & & & & & \\
\hline & & & +0.1 & $101^{\circ} \cdot 78$ & & & & & & & \\
\hline$K$ & 3922.224 & 25488.55 & -0.01 & $131^{\circ} \cdot 105$ & $\mathrm{~K}$ & & $(0)$ & 1.53 & & & \\
\hline \multirow[t]{2}{*}{$c$} & 3920.99 & 25496.6 & +0.2 & $12^{\circ}-18 ?$ & & & & & & & \\
\hline & & & -0.1 & $67^{\circ}-132$ & & & & & & & \\
\hline$C$ & 3920.67 & 25498.7 & +0.1 & $107^{\circ}-129$ & & & & & & & \\
\hline$K$ & 3920.322 & 25500.91 & +0.07 & $148^{\circ} .95$ & $M$ & 5 & $(0.122)$ & 2.038 & 1.184 & 0.940 & \\
\hline$M$ & 3918.285 & 25514.15 & +0.04 & $118^{\circ}-54$ & & & & & & & \\
\hline $\mathrm{K}$ & 3918.021 & 25515.89 & -0.04 & $118^{\circ}-135$ & $\mathrm{~K}$ & & $(0)$ & 1.76 & & & \\
\hline$C$ & 3914.18 & 25540.9 & +0.1 & $59^{\circ}-36$ & & & & & & & \\
\hline
\end{tabular}


Table 6 (cont inued)

\begin{tabular}{|c|c|c|c|c|c|c|c|c|c|c|c|}
\hline \multirow{3}{*}{ Reference } & \multirow{3}{*}{$\begin{array}{c}\text { Wave } \\
\text { Length } \\
\lambda(\AA)\end{array}$} & \multicolumn{2}{|c|}{ Frequency } & \multirow{3}{*}{ Transition } & \multirow{3}{*}{ Reference } & \multirow{2}{*}{\multicolumn{5}{|c|}{ Observed Zeeman Pattern }} & \multirow{3}{*}{$\begin{array}{c}\sigma_{230}-\sigma_{232} \\
\left(\mathrm{~cm}^{-1}\right)\end{array}$} \\
\hline & & $\sigma_{\text {yac }}$ & $\Delta \sigma_{\text {vac }}$ & & & & & & & & \\
\hline & & $\left(\mathrm{cm}^{-1}\right)$ & $\left(\mathrm{cm}^{-1}\right)$ & & & Type & $p$ & $n$ & $g_{1}$ & $g_{2}$ & \\
\hline M & 3913.826 & 25543.25 & +0.02 & $31^{\circ}-14$ & M & 6 & $(0.620)$ & 0.795 & 0.999 & 0.583 & \\
\hline M & 3913.012 & 25548.52 & -0.01 & $99^{\circ} .74$ & L & & $(0)$ & 1.16 & & & \\
\hline M & 3912.285 & 25553.30 & -0.04 & $132^{\circ} \cdot 147$ & $M$ & 6 & $(0.471)$ & 0.987 & 0.932 & 1.037 & \\
\hline M & 3911.308 & 25559.66 & +0.03 & $62^{\circ}-125$ & & & & & & & \\
\hline$v$ & 3908.76 & 25576.3 & -0.1 & $100^{\circ}-153$ & & & & & & & \\
\hline M & 3908.488 & 25578.10 & +0.15 & $64^{\circ} \cdot 41 ?$ & & & & & & & \\
\hline K & 3907.907 & 25581.93 & -0.01 & $154^{\circ}-178$ & K & & $(1.00)$ & 1.18 & & & \\
\hline M & 3907.342 & 25585.60 & -0.05 & $37^{\circ}-53$ & & & & & & & \\
\hline$k$ & 3907.33 & 25585.7 & -0.2 & $93^{\circ} \cdot 63 ?$ & & & & & & & \\
\hline c & 3907.17 & 25586.8 & +0.1 & $113^{\circ}-133$ & & & & & & & \\
\hline k & 3906.81 & 25589.1 & +0.2 & $13^{\circ} \cdot 19 ?$ & & & & & & & \\
\hline $\mathrm{K}$ & 3905.188 & 25599.73 & -0.02 & $134^{\circ}-114$ & L & & (0) & 1.41 & & & 0.37 \\
\hline M & 3904.089 & 25606.94 & -0.12 & $33^{\circ} \cdot 50$ & M & 5 & $(0.090)$ & 1.433 & 0.990 & 1.164 & 0.55 \\
\hline c & 3903.29 & 25612.2 & 0.0 & $102^{\circ}-120$ & & & & & & & \\
\hline K & 3902.123 & 25619.84 & -0.02 & $135^{\circ}-152$ & K & & $(0.86)$ & & & & \\
\hline M & 3901.158 & 25626.18 & -0.02 & $81^{\circ} \cdot 18$ & K & & $(0)$ & 1.43 & & & \\
\hline M & 3900.886 & 25627.98 & -0.12 & $52^{\circ} \cdot 23$ & M & 5 & $(0.094)$ & 1.344 & 1.064 & 0.877 & \\
\hline M & 3900.139 & 25632.88 & -0.05 & $55^{\circ} .71$ & & & & & & & 0.64 \\
\hline v & 3899.71 & 25635.7 & +0.1 & $154^{\circ}-157$ & & & & & & & \\
\hline K & 3899.040 & 25640.10 & +0.11 & $99^{\circ}-115 ?$ & K & & $(0.38)$ & $1.04^{\star}$ & & & \\
\hline \multirow[t]{2}{*}{ M } & 3898.794 & 25641.69 & -0.11 & $100^{\circ}-90 ?$ & & & & & & & \\
\hline & & & +0.14 & $62^{\circ}-40 ?$ & & & & & & & \\
\hline M & 3898.477 & 25643.77 & -0.08 & $58^{\circ}-36$ & & & & & & & \\
\hline C & 3896.53 & 25656.6 & +0.1 & $140^{\circ}-131$ & & & & & & & \\
\hline$c$ & 3895.09 & 25666.1 & 0.0 & $64^{\circ}-128$ & & & & & & & \\
\hline$M$ & 3894.901 & 25667.35 & +0.07 & $3^{\circ}-28$ & & & & & & & \\
\hline$M$ & 3894.416 & 25670.51 & -0.11 & $56^{\circ}-76$ & & & & & & & \\
\hline \multirow[t]{2}{*}{ M } & 3893.396 & 25677.24 & -0.10 & $56^{\circ}-33$ & & & & & & & \\
\hline & & & +0.18 & $133^{\circ}-110 ?$ & & & & & & & \\
\hline M & 3893.113 & 25679.11 & +0.05 & $162^{\circ}-185$ & & & & & & & \\
\hline K & 3893.041 & 25679.61 & -0.08 & $98^{\circ}-110$ & K & & $(0.43)$ & 1.05 & & & \\
\hline
\end{tabular}

*These data are inconsistent with the classifications listed. 
Table 6 (continued)

\begin{tabular}{|c|c|c|c|c|c|c|c|c|c|c|c|}
\hline \multirow{3}{*}{ Reference } & \multirow{3}{*}{$\begin{array}{c}\text { Wave } \\
\text { Length } \\
\lambda(\AA)\end{array}$} & \multicolumn{2}{|c|}{ Frequency } & \multirow{3}{*}{ Transition } & \multirow{3}{*}{ Reference } & \multirow{2}{*}{\multicolumn{5}{|c|}{ Observed Zeeman Pattern }} & \multirow{3}{*}{$\begin{array}{c}\sigma_{230}-\sigma_{232} \\
\left(\mathrm{~cm}^{-1}\right)\end{array}$} \\
\hline & & \multirow{2}{*}{$\begin{array}{c}\sigma_{\mathrm{vac}} \\
\left(\mathrm{cm}^{-1}\right)\end{array}$} & \multirow{2}{*}{$\begin{array}{l}\Delta \sigma_{\mathrm{vac}} \\
\left(\mathrm{cm}^{-1}\right)\end{array}$} & & & & & & & & \\
\hline & & & & & & Type & $p$ & $n$ & $g_{1}$ & $g_{2}$ & \\
\hline$M$ & 3892.311 & 25684.43 & -0.04 & $35^{\circ} \cdot 1$ & $M$ & 4 & $(0.616)$ & 0.279 & 0.892 & 2.122 & \\
\hline $\mathrm{C}$ & 3891.22 & 25691.6 & 0.0 & $133^{\circ}-150$ & & & & & & & \\
\hline K & 3891.065 & 25692.65 & -0.03 & $97^{\circ} .107$ & $\mathrm{~K}$ & & $(0.13)$ & 0.99 & & & \\
\hline$v$ & 3890.83 & 25694.2 & -0.1 & $98^{\circ}-150$ & & & & & & & \\
\hline$M$ & 3890.35 & 25697.4 & +0.1 & $153^{\circ}-154$ & & & & & & & \\
\hline$M$ & 3885.766 & 25727.66 & +0.01 & $111^{\circ}-49$ & & & & & & & \\
\hline K & 3885.64 & 25728.5 & 0.0 & $116^{\circ} .93$ & & & & & & & \\
\hline$M$ & 3885.150 & 25731.74 & -0.02 & $139^{\circ} \cdot 130$ & & & & & & & \\
\hline$k$ & 3884.830 & 25733.89 & -0.03 & $99^{\circ}-151$ & K & & $(0)$ & 0.94 & & & 0.38 \\
\hline \multirow[t]{2}{*}{$M$} & 3884.531 & 25735.87 & -0.03 & $96^{\circ}-145$ & $M$ & 4 & $(0.062)$ & & 1.170 & 1.052 & \\
\hline & & & & & $\mathbf{K}$ & & (0) & 0.67 & & & \\
\hline C & 3882.33 & 25750.5 & 0.0 & $106^{\circ}-88$ & & & & & & & \\
\hline$M$ & 3882.152 & 25751.62 & -0.02 & $78^{\circ}-17$ & $M$ & 5 & $(0.091)$ & 1.415 & 1.148 & 0.969 & \\
\hline$M$ & 3881.490 & 25756.03 & 0.00 & $79^{\circ}-53$ & $M$ & 6 & $(0.108)$ & 1.059 & 1.081 & 1.038 & \\
\hline$k$ & 3879.810 & 25767.18 & -0.02 & $60^{\circ}-82$ & & & & & & & \\
\hline$c$ & 3878.08 & 25778.7 & -0.1 & $18^{\circ}-78$ & & & & & & & \\
\hline$K$ & 3875.53 & 25795.6 & -0.1 & $94^{\circ}-65$ & & & & & & & \\
\hline$v$ & 3874.25 & 25804.2 & -0.1 & $136^{\circ}-154 ?$ & & & & & & & \\
\hline$K$ & 3872.364 & 25816.73 & +0.08 & $1^{\circ} .27$ & $K$ & & $(0.34)$ & & & & \\
\hline $\mathrm{K}$ & 3871.464 & 25822.73 & -0.01 & $164^{\circ}-181$ & $K$ & & (0) & 1.36 & & & \\
\hline$K$ & 3870.94 & 25826.2 & -0.1 & $17^{\circ}-72$ & & & & & & & \\
\hline$M$ & 3869.981 & 25832.60 & -0.05 & $80^{\circ}-93$ & & & & & & & \\
\hline$M$ & 3869.363 & 25836.73 & +0.08 & $27^{\circ}-48$ & & & & & & & \\
\hline $\mathrm{v}$ & 3867.89 & 25846.6 & -0.1 & $2^{\circ}-9$ & & & & & & & \\
\hline V & 3867.59 & 25848.6 & 0.0 & $75^{\circ} \cdot 15$ & & & & & & & \\
\hline$M$ & 3866.894 & 25853.24 & -0.03 & $100^{\circ}-77$ & $M$ & $7 b$ & $(0)$ & 0.864 & 0.864 & 0.864 & \\
\hline$M$ & 3865.036 & 25865.65 & +0.13 & $143^{\circ}-163 ?$ & & & & & & & \\
\hline$c$ & 3865.00 & 25865.9 & 0.0 & $56^{\circ} .116$ & & & & & & & \\
\hline$K$ & 3864.371 & 25870.12 & +0.04 & $8^{\circ} .17$ & & & & & & & \\
\hline V & 3863.83 & 25873.7 & 0.0 & $153^{\circ}-177$ & & & & & & & \\
\hline$M$ & 3863.391 & 25876.69 & +0.11 & $130^{\circ}-100$ & $M$ & $7 b$ & $(0)$ & 0.992 & $(1.018)$ & 1.026 & \\
\hline$K$ & 3862.668 & 25881.53 & -0.05 & $115^{\circ}-92$ & & & & & & & \\
\hline$K$ & 3862.57 & 25882.2 & 0.0 & $99^{\circ}-75$ & & & & & & & \\
\hline
\end{tabular}


Table 6 (continued)

\begin{tabular}{|c|c|c|c|c|c|c|c|c|c|c|c|}
\hline \multirow{3}{*}{ Reference } & \multirow{3}{*}{$\begin{array}{c}\text { Wave } \\
\text { Length } \\
\lambda(\AA)\end{array}$} & \multicolumn{2}{|c|}{ Frequency } & \multirow{3}{*}{ Transition } & \multirow{3}{*}{ Reference } & \multirow{2}{*}{\multicolumn{5}{|c|}{ Observed Zeeman Pattern }} & \multirow{3}{*}{$\begin{array}{c}\sigma_{230}-\sigma_{232} \\
\left(\mathrm{~cm}^{-1}\right)\end{array}$} \\
\hline & & & & & & & & & & & \\
\hline & & $\begin{array}{c}\text { vac } \\
\left(\mathrm{cm}^{-1}\right)\end{array}$ & $\left(\mathrm{cm}^{-1}\right)$ & & & Type & $p$ & $n$ & $g_{1}$ & $g_{2}$ & \\
\hline \multirow[t]{2}{*}{ K } & 3862.411 & 25883.25 & -0.08 & $134^{\circ}-174$ & K & & $(0)$ & 0.49 & & & \\
\hline & & & +0.17 & $152^{\circ}-153 ?$ & & & & & & & \\
\hline$M$ & 3861.547 & 25889.05 & 0.00 & $16^{\circ}-69$ & $M$ & 5 & $(0.108)$ & 1.382 & 0.840 & 1.058 & \\
\hline$M$ & 3859.832 & 25900.55 & +0.06 & $94^{\circ} \cdot 104$ & $M$ & 6 & $(0.304)$ & 1.087 & 1.131 & 1.044 & \\
\hline $\mathrm{K}$ & 3859.760 & 25901.03 & -0.06 & $133^{\circ}-112$ & & & & & & & \\
\hline v & 3857.37 & 25917.1 & +0.1 & $64^{\circ}-88$ & & & & & & & \\
\hline$v$ & 3856.51 & 25922.9 & +0.1 & $50^{\circ}-58$ & & & & & & & \\
\hline C & 3856.18 & 25925.1 & +0.1 & $103^{\circ} \cdot 126$ & & & & & & & \\
\hline v & 3854.89 & 25933.8 & -0.1 & $17^{0}-31$ & K & & $(0.17)$ & 1.14 & & & \\
\hline$M$ & 3854.507 & 25936.33 & 0.00 & $151^{\circ}-147$ & $M$ & 5 & $(0.043)$ & 1.533 & 1.134 & 1.045 & 0.42 \\
\hline$M$ & 3852.962 & 25946.73 & -0.02 & $97^{\circ}-148$ & $M$ & $7 b$ & & 1.051 & $(0.983)$ & 0.999 & \\
\hline $\mathrm{K}$ & 3850.290 & 25964.73 & +0.06 & $17^{\circ}-8$ & & & & & & & \\
\hline$c$ & 3848.83 & 25974.6 & 0.0 & $137^{\circ}-122$ & & & & & & & \\
\hline$k$ & 3846.64 & 25989.4 & 0.0 & $134^{\circ}-115$ & & & & & & & \\
\hline v & 3843.41 & 26011.2 & +0.2 & $83^{\circ}-94 ?$ & & & & & & & \\
\hline$M$ & 3841.964 & 26021.01 & -0.05 & $96^{\circ}-67$ & $M$ & 5 & $(0.110)$ & 1.728 & 1.174 & 0.953 & 0.42 \\
\hline$M$ & 3839.749 & 26036.01 & 0.00 & $130^{\circ} \cdot 101$ & $M$ & 5 & $(0.054)$ & 1.397 & 1.024 & 0.918 & \\
\hline$K$ & 3839.426 & 26038.20 & +0.07 & $155^{\circ}-159$ & $\mathrm{~K}$ & & $(0)$ & 1.11 & & & \\
\hline$M$ & 3837.880 & 26048.67 & +0.02 & $153^{\circ}-155$ & & & & & & & 0.49 \\
\hline$C$ & 3837.81 & 26049.2 & 0.0 & $102^{\circ} \cdot 158$ & & & & & & & \\
\hline $\mathrm{v}$ & 3833.69 & 26077.2 & +0.1 & $99^{\circ} \cdot 152$ & & & & & & & \\
\hline$K$ & 3833.231 & 26080.28 & -0.03 & $154^{\circ}-187$ & & & & & & & \\
\hline$K$ & 3832.789 & 26083.29 & 0.00 & $134^{\circ}-151$ & $\mathrm{~K}$ & & $(0.39)$ & 1.10 & & & \\
\hline$M$ & 3832.431 & 26085.69 & -0.05 & $21^{\circ}-39$ & & & & & & & \\
\hline V & 3832.34 & 26086.3 & 0.0 & $59^{\circ}-119$ & & & & & & & \\
\hline \multirow[t]{2}{*}{$K$} & 3831.737 & 26090.45 & +0.01 & $152^{\circ}-176$ & $L$ & & $(0.24)$ & 1.13 & & & \\
\hline & & & & & $M$ & $\epsilon$ & $(0.237)$ & 1.045 & 1.097 & $(1.140)^{\star}$ & \\
\hline$K$ & 3831.53 & 26091.9 & 0.0 & $135^{\circ}-153$ & & & & & & & \\
\hline
\end{tabular}

*These data are inconsistent with the classifications listed. 
Table 6 (cont inued)

\begin{tabular}{|c|c|c|c|c|c|c|c|c|c|c|c|}
\hline \multirow{3}{*}{ Reference } & \multirow{3}{*}{$\begin{array}{c}\text { Wave } \\
\text { Length } \\
\lambda(\AA)\end{array}$} & \multicolumn{2}{|c|}{ Frequency } & \multirow{3}{*}{ Transition } & \multirow{3}{*}{ Reference } & \multirow{2}{*}{\multicolumn{5}{|c|}{ Observed Zeeman Pattern }} & \multirow{3}{*}{$\begin{array}{c}\sigma_{230}-\sigma_{232} \\
\left(\mathrm{~cm}^{-1}\right)\end{array}$} \\
\hline & & \multirow{2}{*}{$\begin{array}{c}\sigma_{\mathrm{vac}} \\
\left(\mathrm{cm}^{-1}\right)\end{array}$} & \multirow{2}{*}{$\begin{array}{l}\Delta \sigma_{v a c} \\
\left(\mathrm{~cm}^{-1}\right)\end{array}$} & & & & & & & & \\
\hline & & & & & & Type & $p$ & $n$ & $g_{1}$ & $g_{2}$ & \\
\hline V & 3830.51 & 26098.8 & 0.0 & $56^{\circ}-77$ & & & & & & & \\
\hline$M$ & 3829.410 & 26106.27 & +0.01 & $20^{\circ}-82$ & & & & & & & \\
\hline$M$ & 3826.947 & 26123.11 & +0.18 & $43^{\circ}-3$ & $M$ & 4 & $(0.650)$ & 0.601 & 1.251 & 2.551 & \\
\hline V & 3826.47 & 26126.4 & +0.1 & $123^{\circ}-95$ & & & & & & & \\
\hline$K$ & 3825.040 & 26136.13 & -0.04 & $54^{\circ}-107$ & $M$ & 4 & & 0.898 & $(1.088)$ & 1.033 & 0.38 \\
\hline$M$ & 3823.589 & 26146.05 & +0.01 & $119^{\circ}-54$ & $M$ & 4 & $(0.057)$ & 0.848 & 1.136 & 1.252 & 0.42 \\
\hline$M$ & 3823.327 & 26147.81 & -0.05 & $119^{\circ}-135$ & & & & & & & \\
\hline C & 3822.65 & 26152.5 & 0.0 & $103^{\circ}-160$ & & & & & & & \\
\hline \multirow[t]{2}{*}{$K$} & 3822.149 & 26155.91 & -0.05 & $53^{\circ} \cdot 67$ & $M$ & 6 & & 0.769 & 0.595 & 0.950 & \\
\hline & & & & & $\mathrm{K}$ & & $(0.86)$ & 0.77 & & & \\
\hline$M$ & 3821.431 & 26160.81 & +0.03 & $97^{\circ} \cdot 108$ & $M$ & 6 & $(0.352)$ & 0.930 & 0.982 & 0.880 & \\
\hline$M$ & 3819.28 & 26175.5 & 0.0 & $94^{\circ}-144$ & & & & & & & \\
\hline$M$ & 3817.372 & 26188.60 & -0.10 & $108^{\circ} \cdot 130 ?$ & & & & & & & \\
\hline v & 3816.80 & 26192.6 & 0.0 & $115^{\circ}-53$ & & & & & & & \\
\hline \multirow[t]{2}{*}{$K$} & 3816.551 & 26194.26 & -0.08 & $147^{\circ}-135$ & $K$ & & $(0)$ & 1.13 & & & \\
\hline & & & +0.23 & $118^{\circ}-94 ?$ & $K$ & & $(0)$ & 1.13 & & & \\
\hline$K$ & 3815.021 & 26204.76 & +0.05 & $116^{\circ}-134$ & $K$ & & $(0)$ & 1.18 & & & \\
\hline$M$ & 3813.625 & 26214.36 & 0.00 & $36^{\circ}-15$ & $M$ & 6 & $(0.350)$ & 0.915 & 1.032 & 0.799 & \\
\hline $\mathrm{K}$ & 3813.323 & 26216.43 & +0.12 & $64^{\circ}-89 ?$ & & & & & & & \\
\hline$M$ & 3813.064 & 26218.21 & +0.05 & $131^{\circ}-145$ & $M$ & 6 & $(0.124)$ & 1.073 & 1.087 & 1.059 & \\
\hline$M$ & 3811.383 & 26229.75 & +0.02 & $141^{\circ}-132$ & & & & & & & \\
\hline C & 3810.32 & 26237.1 & -0.1 & $132^{\circ}-107$ & & & & & & & \\
\hline $\mathrm{K}$ & 3809.831 & 26240.46 & -0.06 & $152^{\circ} \cdot 186$ & $L$ & & $(0)$ & 1.31 & & & \\
\hline K & 3809.46 & 26243.0 & 0.0 & $98^{\circ}-72$ & & & & & & & \\
\hline $\mathrm{K}$ & 3809.09 & 26245.6 & 0.0 & $16^{\circ}-28$ & & & & & & & \\
\hline$K$ & 3808.69 & 26248.3 & 0.0 & $17^{\circ}-32$ & & & & & & & \\
\hline$M$ & 3808.146 & 26252.05 & -0.07 & $133^{\circ}-172$ & & & & & & & \\
\hline$M$ & 3807.875 & 26253.94 & +0.03 & $95^{\circ}-145$ & $M$ & 5 & $(0.071)$ & 1.566 & 0.921 & 1.064 & \\
\hline$K$ & 3807.51 & 26256.5 & -0.1 & $55^{\circ}-72$ & & & & & & & \\
\hline$M$ & 3805.824 & 26268.09 & -0.08 & $15^{\circ}-23$ & $M$ & 6 & $(0.570)$ & 0.681 & 0.490 & 0.870 & \\
\hline$K$ & 3804.997 & 26273.80 & +0.03 & $82^{\circ}-18$ & $\mathrm{~K}$ & & $(0.11)$ & 1.58 & & & \\
\hline$K$ & 3803.074 & 26287.08 & -0.07 & $131^{\circ} \cdot 168$ & $K$ & & $(0)$ & 0.74 & & & 0.42 \\
\hline$M$ & 3802.150 & 26293.47 & -0.01 & $53^{\circ} \cdot 106$ & $\mathrm{~K}$ & & $(0.20)$ & & & & \\
\hline
\end{tabular}


Table 6 (continued)

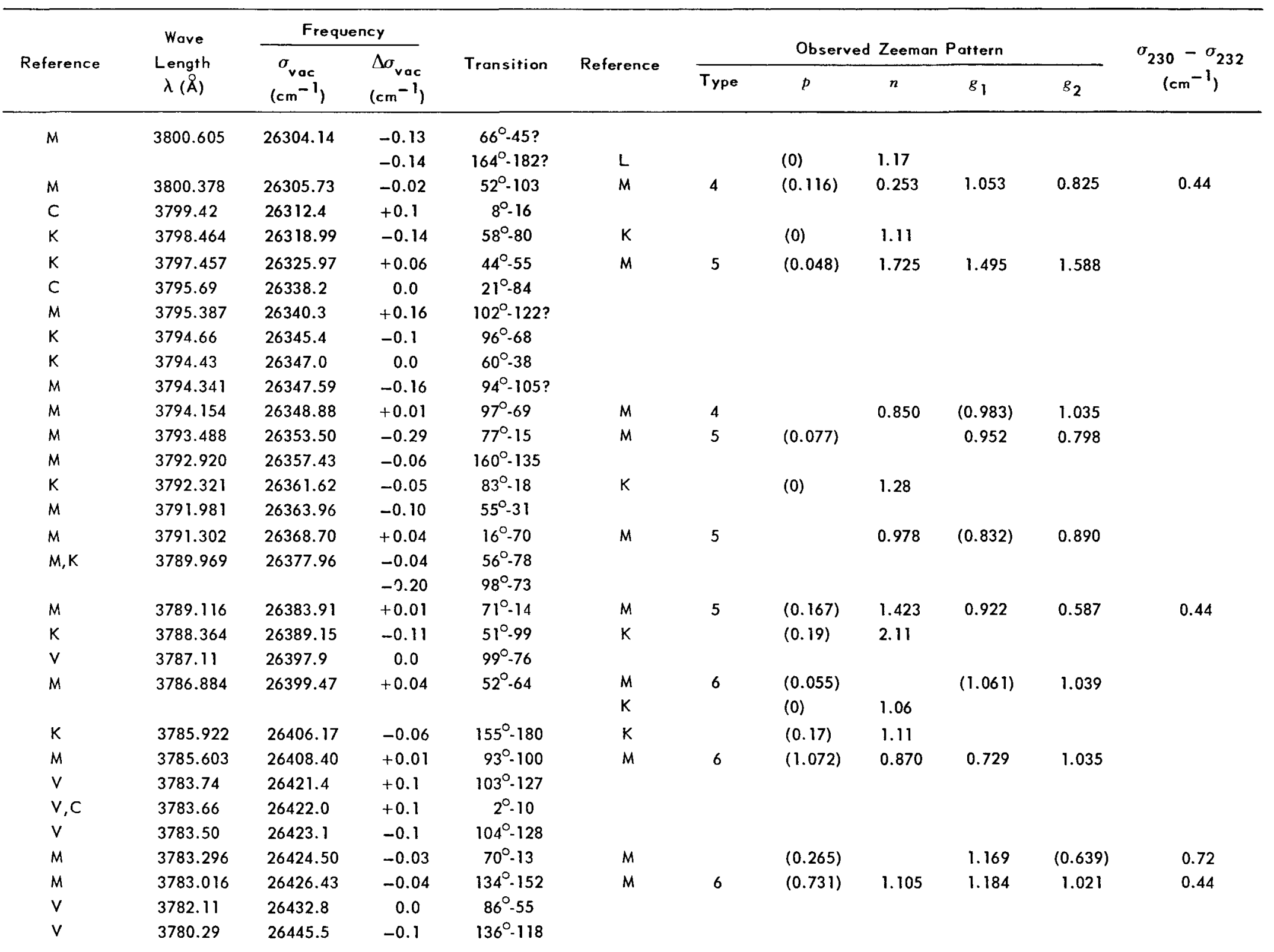


Table 6 (continued)

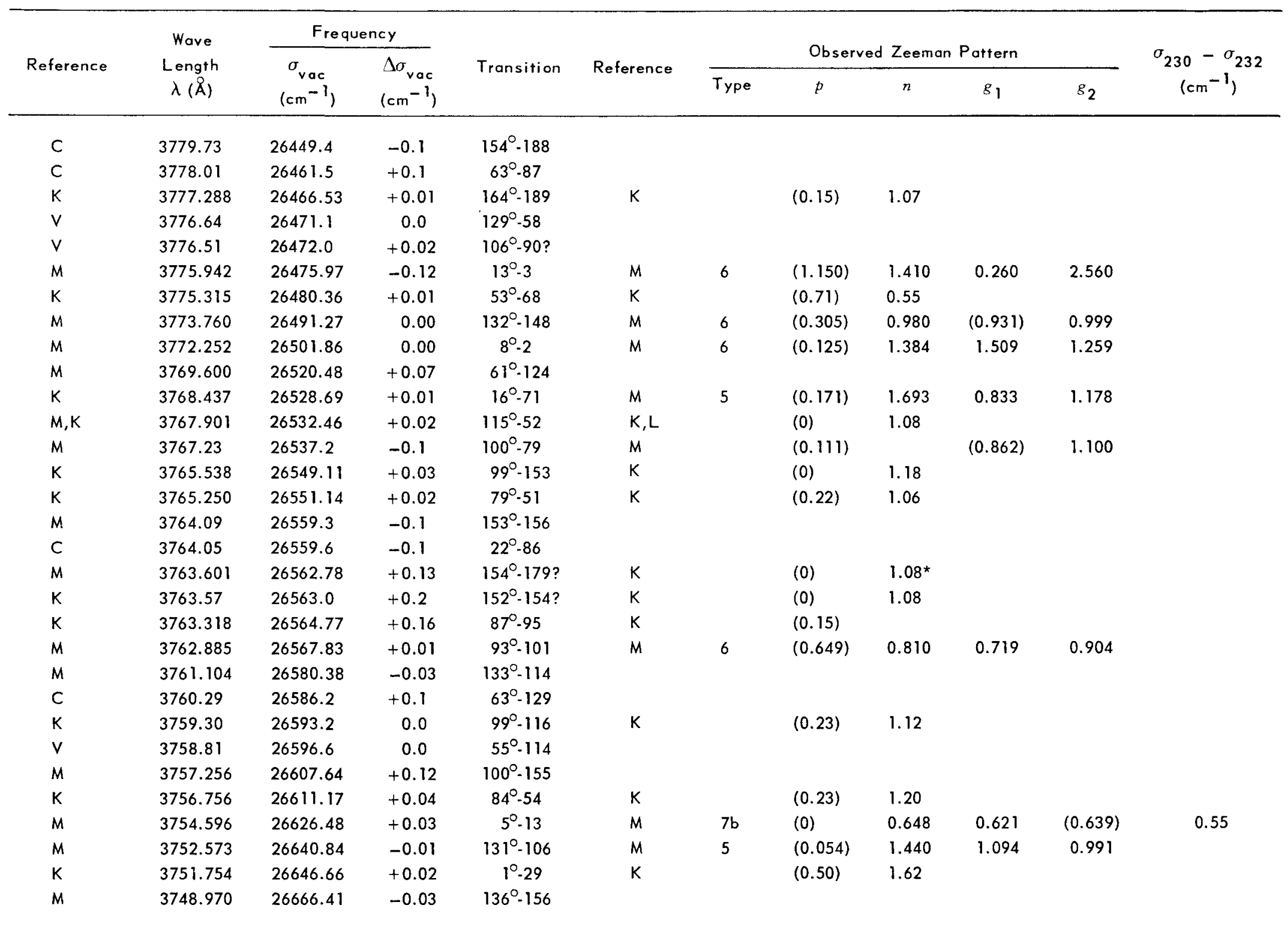


Table 6. (continued)

\begin{tabular}{|c|c|c|c|c|c|c|c|c|c|c|c|}
\hline \multirow{3}{*}{ Reference } & \multirow{3}{*}{$\begin{array}{c}\text { Wave } \\
\text { Length } \\
\lambda(\AA)\end{array}$} & \multicolumn{2}{|c|}{ Frequency } & \multirow{3}{*}{ Transition } & \multirow{3}{*}{ Reference } & \multirow{2}{*}{\multicolumn{5}{|c|}{ Observed Zeeman Pattern }} & \multirow{3}{*}{$\begin{array}{c}\sigma_{230}-\sigma_{232} \\
\left(\mathrm{~cm}^{-1}\right)\end{array}$} \\
\hline & & \multirow{2}{*}{$\begin{array}{c}\sigma_{\mathrm{vac}} \\
\left(\mathrm{cm}^{-1}\right)\end{array}$} & \multirow{2}{*}{$\begin{array}{l}\Delta \sigma_{\mathrm{vac}} \\
\left(\mathrm{cm}^{-1}\right)\end{array}$} & & & & & & & & \\
\hline & & & & & & Type & $p$ & $n$ & $g_{1}$ & $g_{2}$ & \\
\hline K & 3748.280 & 26671.35 & +0.06 & $97^{\circ}-109$ & $\mathrm{~K}$ & & $(0.24)$ & 1.04 & & & 0.45 \\
\hline $\mathrm{C}$ & 3747.92 & 26673.9 & -0.1 & $104^{\circ}-88$ & & & & & & & \\
\hline$M$ & 3747.547 & 26676.57 & -0.03 & $95^{\circ}-106$ & $M$ & 6 & $(0.242)$ & 0.980 & $(0.911)$ & 0.980 & \\
\hline $\mathrm{K}$ & 3746.467 & 26684.26 & -0.01 & $130^{\circ}-142$ & & & & & & & \\
\hline v & 3746.22 & 26686.0 & 0.0 & $20^{\circ}-38$ & & & & & & & \\
\hline$M$ & 3745.983 & 26687.70 & -0.06 & $52^{\circ}-24$ & $M$ & 5 & $(0.117)$ & 1.419 & 1.064 & 0.828 & \\
\hline \multirow[t]{2}{*}{$M$} & 3744.739 & 26696.57 & -0.01 & $79^{\circ}-17$ & $M$ & & $(0.053)$ & & 1.076 & $(0.968)$ & \\
\hline & & & & & $\mathrm{K}$ & & $(0)$ & 1.20 & & & \\
\hline $\mathrm{K}$ & 3744.657 & 26697.16 & +0.02 & $100^{\circ}-117$ & $\mathrm{~K}$ & & $(0.46)$ & & & & \\
\hline \multirow[t]{2}{*}{$K$} & 3743.506 & 26705.36 & +0.06 & $132^{\circ}-108$ & $L$ & & $(0)$ & 1.12 & & & \\
\hline & & & -0.07 & $139^{\circ} \cdot 162$ & & & & & & & \\
\hline$K$ & 3742.230 & 26714.47 & +0.02 & $146^{\circ}-93$ & & & & & & & \\
\hline$M$ & 3741.190 & 26721.89 & -0.04 & $71^{\circ}-49$ & $M$ & 6 & $(0.380)$ & 0.993 & 0.917 & 1.070 & 0.36 \\
\hline$M$ & 3740.849 & 26724.30 & 0.00 & $97^{\circ}-149$ & & & & & & & 0.46 \\
\hline$M$ & 3738.846 & 26738.64 & 0.00 & $9^{\circ} \cdot 17$ & $M$ & $7 b$ & (0) & 0.968 & 0.968 & 0.968 & \\
\hline \multirow[t]{2}{*}{$\mathrm{K}$} & 3738.750 & 26739.33 & +0.08 & $152^{\circ}-177$ & $k$ & & (0) & 1.17 & & & \\
\hline & & & -0.06 & $141^{\circ} \cdot 163$ & & & & & & & \\
\hline V & 3738.11 & 26743.9 & 0.0 & $114^{\circ} \cdot 133$ & & & & & & & \\
\hline v & 3737.65 & 26747.2 & 0.0 & $101^{\circ} \cdot 157$ & & & & & & & \\
\hline$M$ & 3734.601 & 26769.04 & 0.00 & $51^{\circ}-62$ & $M$ & 6 & $(0.180)$ & 0.840 & $(0.857)$ & 0.785 & 0.78 \\
\hline v & 3732.70 & 26782.7 & -0.1 & $56^{\circ}-79$ & & & & & & & \\
\hline v & 3731.44 & 26791.7 & -0.1 & $18^{\circ}-37$ & $M$ & 6 & $(0.404)$ & 1.227 & 1.365 & 1.095 & \\
\hline$K$ & 3731.36 & 26792.3 & 0.0 & $54^{\circ}-69$ & & & & & & & \\
\hline$M$ & 3730.87 & 26795.8 & 0.0 & $16^{\circ}-29$ & & & & & & & \\
\hline$K$ & 3730.754 & 26796.64 & 0.00 & $153^{\circ}-178$ & $M$ & 6 & $(0.596)$ & 1.220 & 1.266 & 1.159 & 0.46 \\
\hline$M$ & 3728.12 & 26815.6 & +0.1 & $63^{\circ}-42$ & & & & & & & \\
\hline$M$ & 3726.730 & 26825.57 & -0.04 & $73^{\circ}-91$ & $M$ & $7 b$ & $(0)$ & 1.257 & $(1.232)$ & 1.224 & \\
\hline K & 3726.669 & 26826.01 & +0.05 & $119^{\circ}-94$ & $K$ & & (0) & 1.13 & & & \\
\hline \multirow[t]{2}{*}{$M$} & 3723.655 & 26847.73 & 0.00 & $112^{\circ}-91$ & $M$ & 6 & $(0.102)$ & & 1.203 & $(1.232)$ & 0.45 \\
\hline & & & & & $\mathrm{K}$ & & $(0.09)$ & 1.19 & & & \\
\hline
\end{tabular}


Table 6 (continued)

\begin{tabular}{|c|c|c|c|c|c|c|c|c|c|c|c|}
\hline \multirow{3}{*}{ Reference } & \multirow{3}{*}{$\begin{array}{c}\text { Wave } \\
\text { Length } \\
\lambda(\AA)\end{array}$} & \multicolumn{2}{|c|}{ Frequency } & \multirow{3}{*}{ Transition } & \multirow{3}{*}{ Reference } & \multirow{2}{*}{\multicolumn{5}{|c|}{ Observed Zeeman Pattern }} & \multirow{3}{*}{$\begin{array}{c}\sigma_{230}-\sigma_{232} \\
\left(\mathrm{~cm}^{-1}\right)\end{array}$} \\
\hline & & \multirow{2}{*}{$\begin{array}{c}\sigma_{\mathrm{vac}} \\
\left(\mathrm{cm}^{-1}\right)\end{array}$} & \multirow{2}{*}{$\begin{array}{c}\Delta \sigma_{\mathrm{Vac}} \\
\left(\mathrm{cm}^{-1}\right)\end{array}$} & & & & & & & & \\
\hline & & & & & & Type & $p$ & $n$ & $g_{1}$ & $g_{2}$ & \\
\hline $\mathrm{K}$ & 3723.294 & 26850.33 & -0.01 & $153^{\circ} \cdot 157$ & $\mathrm{~K}$ & & $(0)$ & $1.73^{\star}$ & & & \\
\hline \multirow[t]{2}{*}{ M } & 3722.117 & 26858.82 & 0.00 & $73^{\circ}-50$ & $M$ & 6 & $(0.137)$ & & 1.218 & $(1.163)$ & \\
\hline & & & & & K & & $(0.12)$ & 1.19 & & & \\
\hline M & 3721.831 & 26860.88 & -0.04 & $32^{\circ}-14$ & $M$ & 6 & $(0.865)$ & 0.869 & 1.169 & 0.591 & \\
\hline K & 3721.487 & 26863.37 & -0.10 & $95^{\circ} \cdot 68$ & & & & & & & \\
\hline M & 3721.402 & 26863.96 & -0.03 & $133^{\circ}-174$ & & & & & & & \\
\hline V & 3720.71 & 26869.0 & -0.1 & $54^{\circ}-27$ & & & & & & & \\
\hline$M$ & 3720.309 & 26871.87 & -0.01 & $116^{\circ}-92$ & $M$ & 6 & $(0.650)$ & & 1.142 & 0.956 & \\
\hline $\mathrm{K}$ & 3720.22 & 26872.5 & +0.1 & $147^{\circ}-94$ & & & & & & & \\
\hline \multirow[t]{2}{*}{$K$} & 3719.979 & 26874.26 & 0.00 & $151^{\circ}=148$ & $K$ & & $(0)$ & 1.68 & & & \\
\hline & & & & & $M$ & 5 or 4 & & & & & \\
\hline $\mathrm{K}$ & 3719.697 & 26876.30 & +0.01 & $44^{\circ}-19$ & $\mathrm{~K}$ & & $(0.17)$ & 1.48 & & & \\
\hline $\mathrm{V}$ & & & -0.2 & $112^{\circ}-50 ?$ & & & & & & & \\
\hline $\mathrm{K}$ & 3718.172 & 26887.32 & -0.07 & $131^{\circ}-147$ & $K$ & & $(0.21)$ & 1.07 & & & 0.39 \\
\hline$M$ & 3717.832 & 26889.76 & +0.06 & $2^{\circ}-35$ & $M$ & 5 & $(0.258)$ & 1.288 & 0.518 & 1.032 & \\
\hline C & 3717.41 & 26892.8 & +0.1 & $25^{\circ}-48$ & & & & & & & \\
\hline \multirow[t]{2}{*}{$K$} & 3716.78 & 26897.4 & 0.0 & $100^{\circ}-118$ & & & & & & & \\
\hline & & & +0.2 & $102^{\circ}-123^{2}$ & & & & & & & \\
\hline$K$ & 3716.56 & 26899.0 & -0.1 & $101^{\circ}-80$ & & & & & & & \\
\hline$M$ & 3716.255 & 26901.16 & -0.06 & $66^{\circ}-46$ & & & & & & & \\
\hline \multirow[t]{2}{*}{$\mathrm{K}$} & 3715.92 & 26903.6 & +0.1 & $129^{\circ}-57$ & & & & & & & \\
\hline & & & -0.1 & $136^{\circ}-178$ & & & & & & & \\
\hline V & 3715.71 & 26905.1 & +0.1 & $137^{\circ}-180$ & & & & & & & \\
\hline C & 3714.45 & 26914.3 & +0.1 & $152^{\circ} \cdot 155$ & & & & & & & \\
\hline C & 3714.31 & 26915.3 & -0.1 & $61^{\circ}-126$ & & & & & & & \\
\hline $\mathrm{K}$ & 3713.90 & 26918.2 & 0.0 & $163^{\circ}-181$ & & & & & & & \\
\hline$M$ & 3712.538 & 26928.12 & +0.12 & $60^{\circ}-83$ & $M$ & 6 & $(0.842)$ & 1.342 & 1.511 & 1.174 & 0.5 \\
\hline V & 3711.80 & 26933.5 & -0.1 & $121^{\circ}-54$ & & & & & & & \\
\hline
\end{tabular}


Table 6 (cont inued)

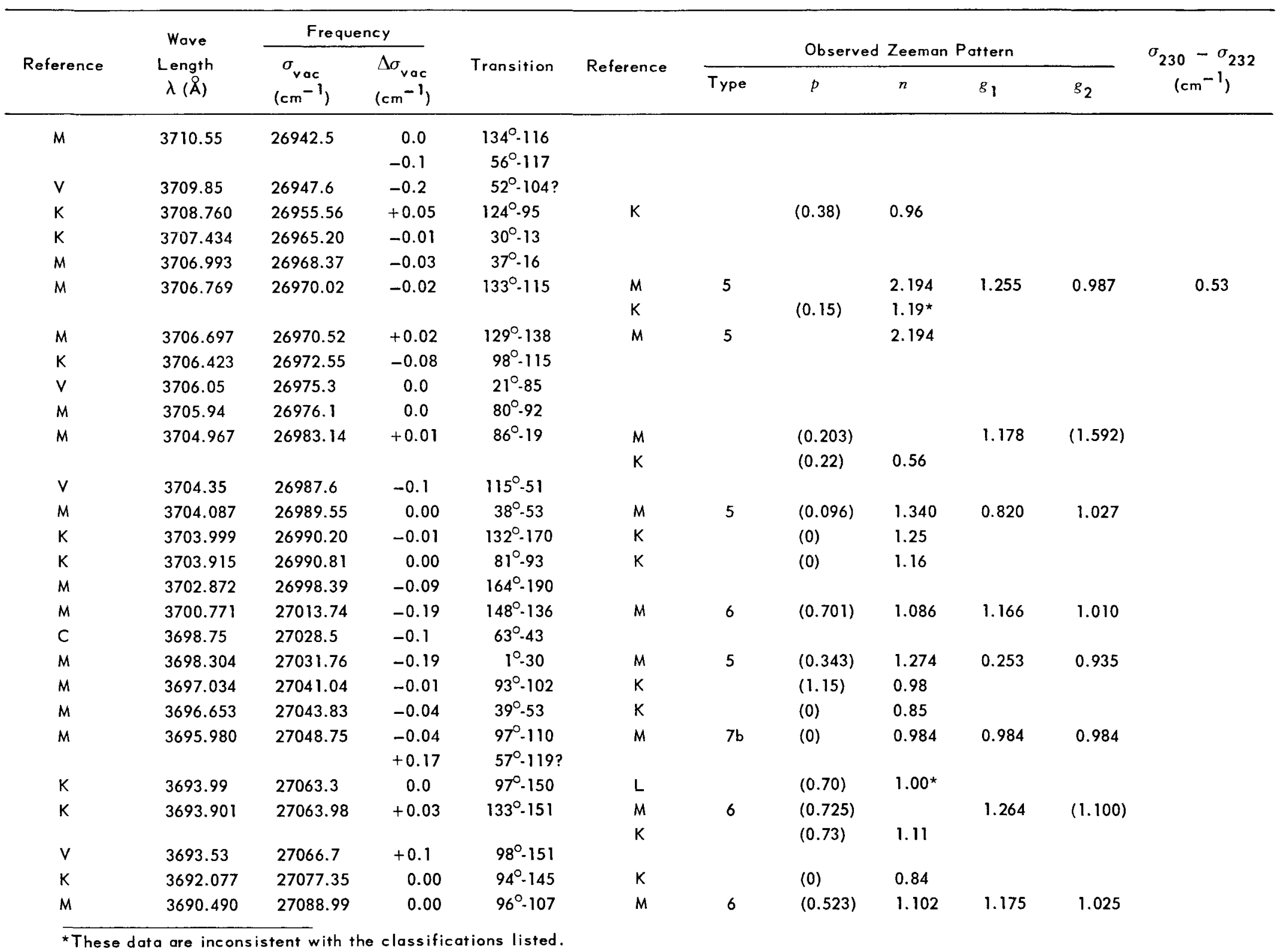


Table 6 (continued)

\begin{tabular}{|c|c|c|c|c|c|c|c|c|c|c|c|}
\hline \multirow{3}{*}{ Reference } & \multirow{3}{*}{$\begin{array}{c}\text { Wave } \\
\text { Length } \\
\lambda(\AA)\end{array}$} & \multicolumn{2}{|c|}{ Frequency } & \multirow{3}{*}{ Transition } & \multirow{3}{*}{ Reference } & \multirow{2}{*}{\multicolumn{5}{|c|}{ Observed Zeeman Pattern }} & \multirow{3}{*}{$\begin{array}{c}\sigma_{230}-\sigma_{232} \\
\left(\mathrm{~cm}^{-1}\right)\end{array}$} \\
\hline & & \multirow{2}{*}{$\begin{array}{c}\sigma_{\text {voc }} \\
\left(\mathrm{cm}^{-1}\right)\end{array}$} & \multirow{2}{*}{$\begin{array}{l}\Delta \sigma_{\text {vac }} \\
\left(\mathrm{cm}^{-1}\right)\end{array}$} & & & & & & & & \\
\hline & & & & & & Type & $p$ & $n$ & $g_{1}$ & $g_{2}$ & \\
\hline$M$ & 3690.119 & 27091.68 & -0.13 & $59^{\circ}-82 ?$ & & & & & & & 0.38 \\
\hline$M$ & 3688.273 & 27105.24 & +0.02 & $99^{\circ}-78$ & & & & & & & \\
\hline K & 3688.200 & 27105.81 & 0.00 & $134^{\circ}-176$ & & & & & & & \\
\hline \multirow[t]{2}{*}{ k } & 3687.525 & 27110.77 & +0.08 & $1^{\circ}-31$ & $k$ & & $(0.40)$ & & & & \\
\hline & & & -0.02 & $59^{\circ} \cdot 122$ & & & & & & & \\
\hline$M$ & 3686.987 & 27114.73 & -0.01 & $54^{\circ} \cdot 109$ & K & & $(0)$ & 1.00 & & & 0.46 \\
\hline$M$ & 3685.871 & 27122.91 & -0.06 & $135^{\circ}-155$ & & & & & & & \\
\hline $\mathrm{C}$ & 3683.73 & 27138.7 & -0.1 & $79^{\circ}-16$ & & & & & & & \\
\hline \multirow[t]{2}{*}{ M } & 3683.332 & 27141.63 & +0.03 & $1^{\circ}-8$ & M & 6 & $(0.346)$ & & $(0.255)$ & 0.947 & \\
\hline & & & & & $\mathrm{K}$ & & $(0.34)$ & 0.60 & & & \\
\hline $\mathrm{K}$ & 3682.346 & 27148.90 & 0.00 & $54^{\circ}-28$ & K & & $(0)$ & 1.03 & & & \\
\hline$K$ & 3681.886 & 27152.31 & +0.01 & $16^{\circ}-72$ & $M$ & 5 & $(0.212)$ & 1.892 & 0.833 & 1.258 & \\
\hline V & 3680.53 & 27162.3 & -0.1 & $108^{\circ} \cdot 162$ & & & & & & & \\
\hline$M$ & 3679.712 & 27168.31 & +0.01 & $162^{\circ}-176$ & $M$ & 5 & $(0.045)$ & 1.746 & 1.251 & 1.161 & 0.42 \\
\hline$M$ & 3678.791 & 27175.14 & -0.06 & $97^{\circ}-111$ & & & & & & & \\
\hline \multirow[t]{2}{*}{$M$} & 3678.029 & 27180.77 & -0.07 & $9^{\circ} .16$ & $M$ & 5 & $(0.316)$ & 1.920 & 0.975 & 1.605 & \\
\hline & & & +0.09 & $40^{\circ}-53$ & $M$ & 4 & $(0.038)$ & 0.927 & 1.117 & 1.041 & \\
\hline$V$ & 3677.94 & 27181.4 & -0.1 & $45^{\circ}-19$ & & & & & & & \\
\hline$M$ & 3677.741 & 27182.87 & -0.02 & $116^{\circ}-53$ & & & & & & & \\
\hline $\mathrm{K}$ & 3676.689 & 27190.67 & 0.00 & $146^{\circ}-134$ & $\mathrm{~K}$ & & $(0.65)$ & 1.13 & & & \\
\hline$c$ & 3676.11 & 27195.0 & +0.1 & $58^{\circ}-82$ & & & & & & & \\
\hline$M$ & 3675.571 & 27198.94 & -0.01 & $32^{\circ}-49$ & $M$ & 4 & $(0.042)$ & 0.951 & 1.159 & 1.074 & \\
\hline$v$ & 3674.02 & 27210.4 & -0.2 & $78^{\circ} \cdot 15 ?$ & & & & & & & \\
\hline$M$ & 3673.799 & 27212.06 & -0.06 & $52^{\circ}-66$ & $M$ & 6 & $(0.140)$ & 1.029 & 1.057 & 1.001 & \\
\hline$c$ & 3673.61 & 27213.5 & +0.2 & $22^{\circ}-41 ?$ & & & & & & & \\
\hline \multirow[t]{2}{*}{$M, K$} & 3673.260 & 27216.05 & 0.00 & $93^{\circ}-142$ & $M$ & & $(0.218)$ & & $(0.718)$ & 1.154 & \\
\hline & & & & & $K$ & & $(0.20)$ & 2.56 & & & \\
\hline$K$ & 3671.527 & 27228.90 & +0.07 & $99^{\circ}-154$ & $K$ & & $(0)$ & 1.01 & & & \\
\hline$M$ & 3670.635 & 27235.52 & 0.00 & $72^{\circ}-14$ & & & & & & & \\
\hline$M$ & 3670.062 & 27239.77 & +0.01 & $34^{\circ}-50$ & $M$ & 5 & $(0.154)$ & 1.613 & 0.865 & 1.165 & \\
\hline
\end{tabular}


Table 6 (continued)

\begin{tabular}{|c|c|c|c|c|c|c|c|c|c|c|c|}
\hline \multirow{3}{*}{ Reference } & \multirow{3}{*}{$\begin{array}{c}\text { Wove } \\
\text { Length } \\
\lambda(\AA)\end{array}$} & \multicolumn{2}{|c|}{ Frequency } & \multirow{3}{*}{ Transition } & \multirow{3}{*}{ Reference } & \multirow{2}{*}{\multicolumn{5}{|c|}{ Observed Zeeman Pattern }} & \multirow{3}{*}{$\begin{array}{c}\sigma_{230}-\sigma_{232} \\
\left(\mathrm{~cm}^{-1}\right)\end{array}$} \\
\hline & & $\sigma_{\text {vac }}$ & $\Delta \sigma_{\text {vac }}$ & & & & & & & & \\
\hline & & $\left(\begin{array}{c}\text { vac } \\
\left(\mathrm{cm}^{-1}\right)\end{array}\right.$ & $\left(\mathrm{cm}^{-1}\right)$ & & & Type & $p$ & $n$ & $g_{1}$ & $g_{2}$ & \\
\hline M & 3669.413 & 27244.55 & -0.05 & $154^{\circ}-159$ & & & & & & & \\
\hline$M$ & 3666.380 & 27267.10 & +0.04 & $20^{\circ} \cdot 83$ & & & & & & & \\
\hline \multirow[t]{2}{*}{$M$} & 3665.731 & 27271.95 & +0.02 & $54^{\circ}-70$ & $M$ & 6 & $(0.486)$ & 0.997 & 1.095 & 0.900 & \\
\hline & & & & & $K$ & & $(0.45)$ & $1.27^{\star}$ & & & \\
\hline \multirow[t]{2}{*}{$\mathrm{K}$} & 3665.174 & 27276.10 & +0.09 & $136^{\circ}-120$ & $M$ & & & 0.641 & 0.982 & $(1.080)$ & \\
\hline & & & & & K & & $(0.08)$ & & & & \\
\hline$M$ & 3663.708 & 27287.01 & -0.04 & $80^{\circ}-53$ & $M$ & $7 b$ & $(0)$ & 1.039 & 1.039 & 1.039 & 0.50 \\
\hline$M$ & 3663.204 & 27290.76 & -0.02 & $16^{\circ}-8$ & & & & & & & \\
\hline K & 3663.152 & 27291.15 & +0.10 & $84^{\circ}-94$ & & & & & & & \\
\hline$M$ & 3662.639 & 27294.94 & -0.07 & $153^{\circ}-187$ & & & & & & & \\
\hline$M$ & 3662.194 & 27298.26 & -0.08 & $101^{\circ}-121$ & & & & & & & \\
\hline$c$ & 3660.77 & 27308.9 & +0.1 & $138^{\circ}-131$ & & & & & & & \\
\hline \multirow[t]{2}{*}{$M$} & 3659.513 & 27318.29 & -0.09 & $140^{\circ}-132$ & $M$ & 6 & $(0.669)$ & 1.182 & 1.230 & 1.129 & 0.43 \\
\hline & & & -0.05 & $162^{\circ} \cdot 186$ & & & & & & & \\
\hline \multirow[t]{2}{*}{$M$} & 3658.235 & 27327.83 & 0.00 & $15^{\circ}-24$ & $M$ & 6 & $(0.500)$ & & $(0.492)$ & 0.825 & \\
\hline & & & & & $K$ & & $(0.50)$ & $0.55^{\star}$ & & & \\
\hline$M$ & 3658.069 & 27329.07 & +0.02 & $8^{\circ}-15$ & $M$ & 4 & $(0.353)$ & 0.441 & 1.506 & 0.798 & \\
\hline$M$ & 3657.538 & 27333.02 & -0.16 & $62^{\circ}-87 ?$ & & & & & & & \\
\hline \multirow[t]{2}{*}{$M$} & 3656.204 & 27343.01 & -0.05 & $96^{\circ}-148$ & $M$ & 4 & $(0.088)$ & 0.394 & 1.179 & 1.005 & \\
\hline & & & & & $K$ & & $(0.32)$ & $0.90^{\star}$ & & & \\
\hline \multirow[t]{2}{*}{$\mathrm{K}$} & 3654.572 & 27355.22 & +0.03 & $155^{\circ}-160$ & $M$ & & $(0.09)$ & & & & \\
\hline & & & & & $K$ & & & 0.68 & & & \\
\hline$M$ & 3653.59 & 27362.6 & +0.1 & $94^{\circ}-67$ & $M$ & & & & (1.132) & 0.953 & \\
\hline$M$ & 3652.542 & 27370.42 & 0.00 & $9^{\circ}-2$ & $M$ & 6 & $(0.139)$ & 1.113 & 0.974 & 1.252 & 0.45 \\
\hline$M$ & 3652.168 & 27373.23 & +0.02 & $151^{\circ}-170$ & $M$ & 6 & $(0.606)$ & 1.125 & 1.070 & $1.180 ?$ & \\
\hline v & 3651.82 & 27375.8 & +0.1 & $17^{\circ}-34$ & & & & & & & \\
\hline V & 3651.51 & 27378.2 & 0.0 & $117^{\circ}-134$ & & & & & & & \\
\hline$M$ & 3650.771 & 27383.70 & -0.03 & $39^{\circ}-52$ & $M$ & 5 & $(0.120)$ & 1.787 & 1.181 & 1.423 & 0.43 \\
\hline$M$ & 3648.639 & 27399.67 & -0.05 & $163^{\circ}-182$ & & & & & & & \\
\hline
\end{tabular}

*These data are inconsistent with the classifications listed. 
Table 6 (continued)

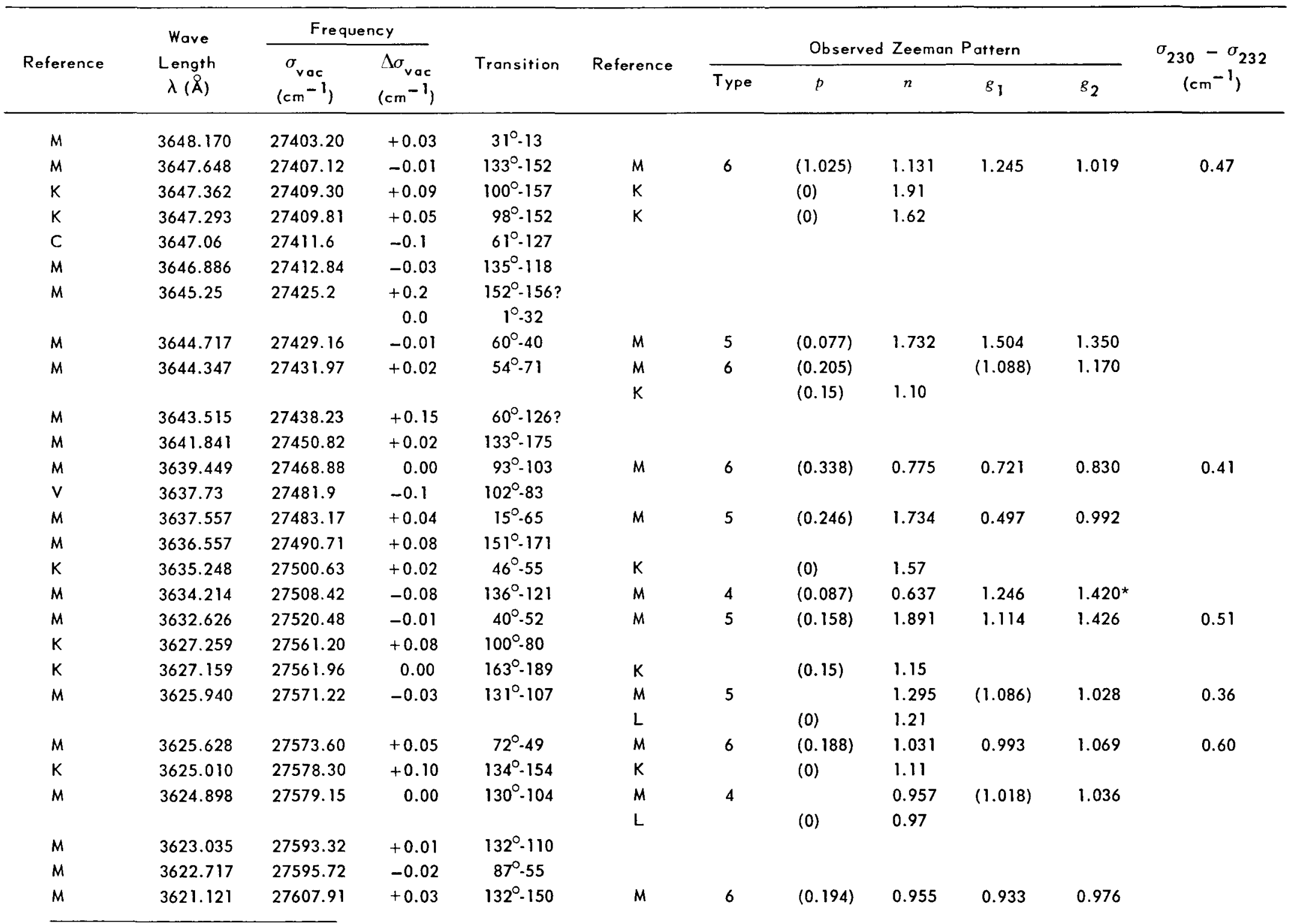

*These data are inconsistent with the classifications listed. 


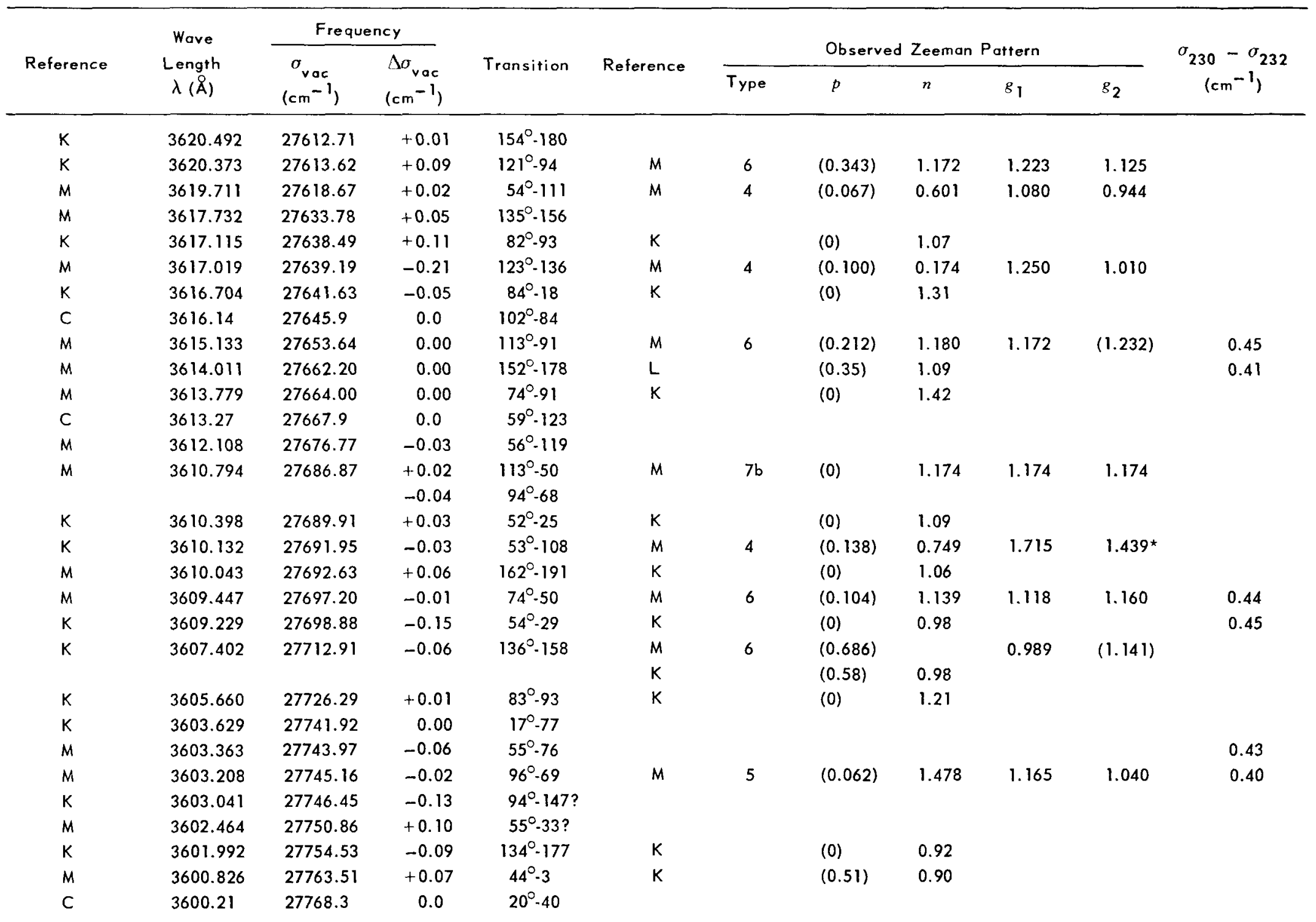

*These data are inconsistent with the classifications listed. 
Table 6 (continued)

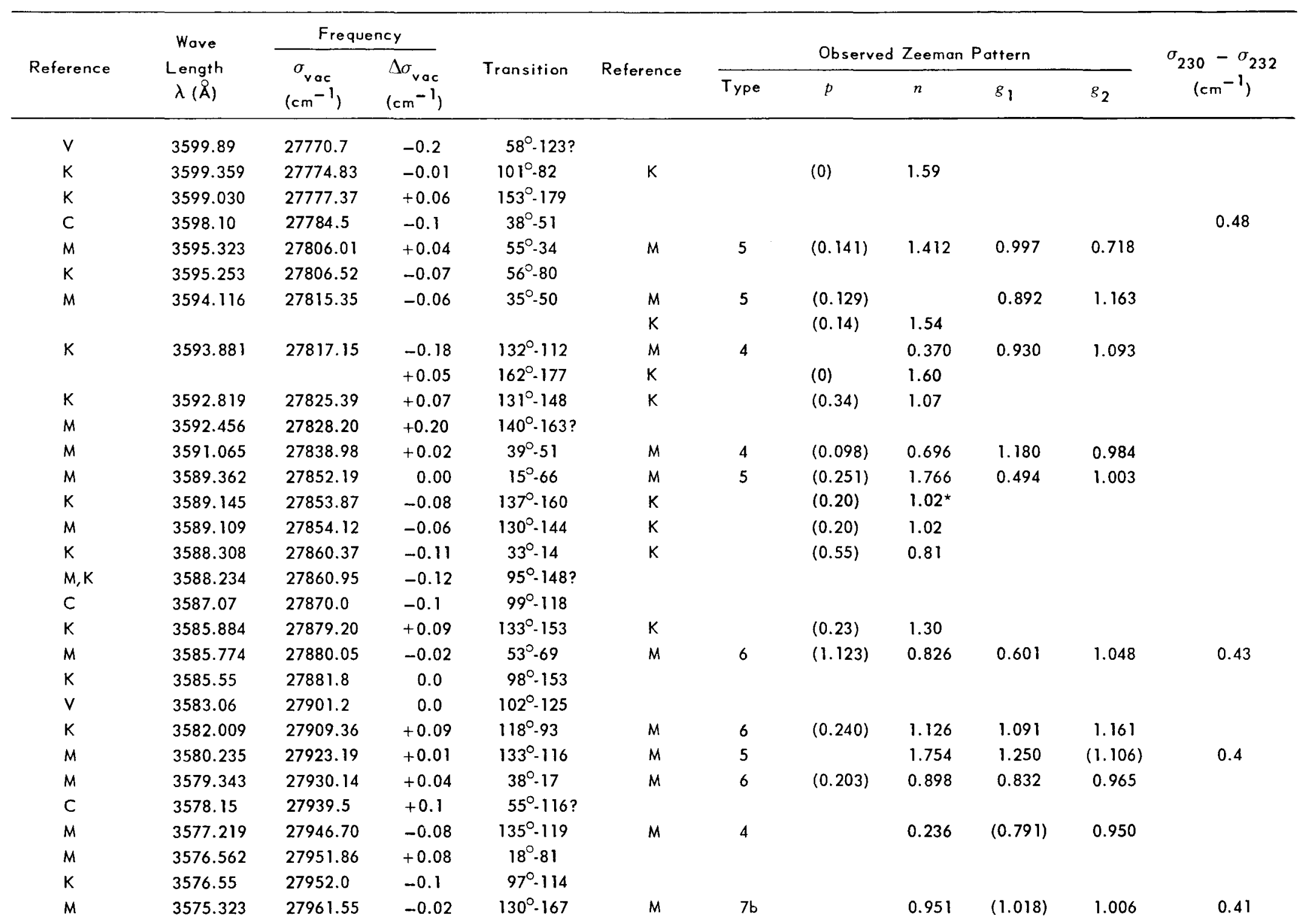

*These data are inconsistent with the classifications listed. 
Table 6 (cont inued)

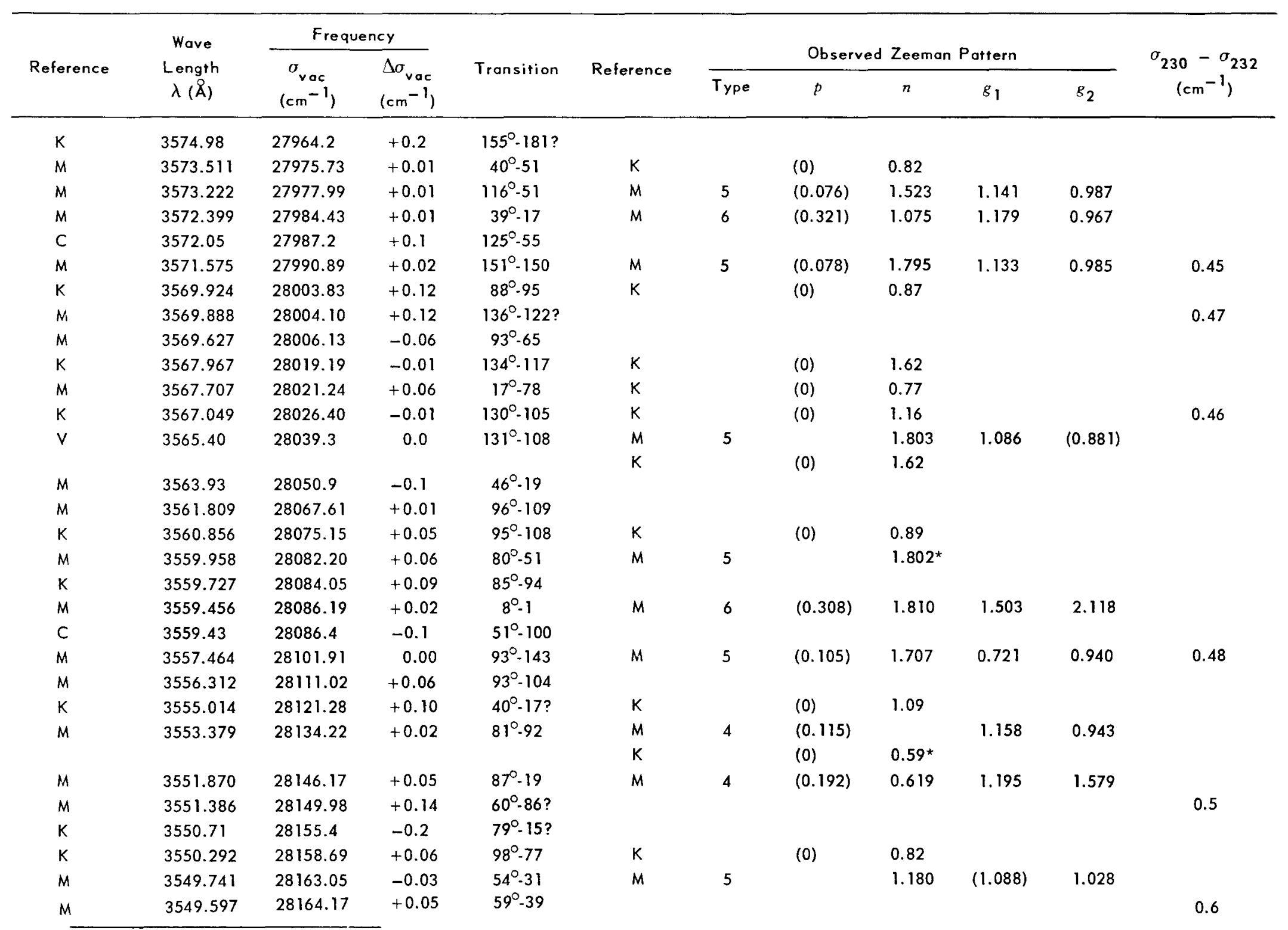

*These data are inconsistent with the classifications listed. 
Table 6 (contınued)

\begin{tabular}{|c|c|c|c|c|c|c|c|c|c|c|c|}
\hline \multirow{3}{*}{ Reference } & \multirow{3}{*}{$\begin{array}{c}\text { Wave } \\
\text { Length } \\
\lambda(\AA)\end{array}$} & \multicolumn{2}{|c|}{ Frequency } & \multirow{3}{*}{ Transition } & \multirow{3}{*}{ Reference } & \multirow{2}{*}{\multicolumn{5}{|c|}{ Observed Zeeman Pattern }} & \multirow{3}{*}{$\begin{array}{c}\sigma_{230}-\sigma_{232} \\
\left(\mathrm{~cm}^{-1}\right)\end{array}$} \\
\hline & & \multirow{2}{*}{$\begin{array}{c}\sigma_{\mathrm{vac}} \\
\left(\mathrm{cm}^{-1}\right)\end{array}$} & \multirow{2}{*}{$\begin{array}{l}1 \sigma_{\text {vac }} \\
\left(\mathrm{cm}^{-1}\right)\end{array}$} & & & & & & & & \\
\hline & & & & & & Type & $p$ & $n$ & $g_{1}$ & $g_{2}$ & \\
\hline$M$ & 3546260 & 2819070 & -001 & $54^{\circ}-73$ & & & & & & & \\
\hline $\mathrm{K}$ & 3545384 & 2819766 & +005 & $9^{\circ} 15$ & $\mathrm{~K}$ & & $(0)$ & 110 & & & \\
\hline$M$ & 3545290 & 2819841 & -010 & $33^{\circ} 49$ & $\mathrm{~K}$ & & (0) & 115 & & & 043 \\
\hline $\mathrm{K}$ & 3545174 & 2819933 & +001 & $161^{\circ} 135$ & $\mathrm{~K}$ & & $(0)$ & 128 & & & \\
\hline $\mathrm{C}$ & 354498 & 282009 & +01 & $41^{\circ} 53$ & & & & & & & \\
\hline$M$ & 354436 & 282058 & 00 & $56^{\circ} 121$ & & & & & & & \\
\hline \multirow[t]{4}{*}{ M } & 3542632 & 2821954 & -004 & $102^{\circ} \cdot 160$ & $M$ & 4 or 5 & $\left(\begin{array}{lll}0 & 13 & 1\end{array}\right)$ & & (0953) & 1215 & \\
\hline & & & & & K & & $(0)$ & $178^{\star}$ & & & \\
\hline & & & +006 & $134^{\circ} 118$ & M & 4 or 5 & $\left(\begin{array}{lll}0 & 131\end{array}\right)$ & & $\left(\begin{array}{ll}1 & 185\end{array}\right)$ & 0923 & \\
\hline & & & & & $K$ & & $(0)$ & $178^{\star}$ & & & \\
\hline$M$ & 3541977 & 2822479 & 000 & $96^{\circ} 70$ & $K$ & & $\left(\begin{array}{ll}0 & 40\end{array}\right)$ & $188^{*}$ & & & \\
\hline$M$ & 3541622 & 2822761 & +001 & $80^{\circ} 17$ & $M$ & 5 & & 1145 & 1037 & $(0968)$ & \\
\hline$M$ & 3539589 & 2824383 & -001 & $71^{\circ} 13$ & $M$ & 5 & $\left(\begin{array}{ll}0 & 140\end{array}\right)$ & 1346 & 0920 & 0636 & 043 \\
\hline$M$ & 3539325 & 2824593 & +002 & $51^{\circ} 101$ & $M$ & 5 & & 0996 & $(0857)$ & 0897 & 059 \\
\hline$M$ & 3537161 & 2826321 & +002 & $95^{\circ} 69$ & $M$ & 4 & $(0066)$ & 0577 & 0909 & 1043 & 035 \\
\hline$M$ & 353665 & 282673 & +01 & $58^{\circ}-39$ & & & & & & & \\
\hline$M$ & 3535336 & 2827778 & -006 & $108^{\circ} 132$ & & & & & & & \\
\hline$M$ & 3533706 & 2829082 & +004 & $132^{\circ} 173$ & & & & & & & \\
\hline$C$ & 353188 & 283055 & 00 & $100^{\circ} 81$ & & & & & & & \\
\hline$k$ & 3528948 & 2832899 & -001 & $151^{\circ} \cdot 184$ & $K$ & & $(0)$ & 103 & & & 047 \\
\hline $\mathrm{K}$ & 3528823 & 2832999 & +004 & $15^{\circ}-25$ & $M$ & 6 & $(0814)$ & 0766 & 0495 & 1042 & \\
\hline$M$ & 3527350 & 2834181 & +015 & $97^{\circ} 115^{?}$ & $K$ & & $(0)$ & 101 & & & \\
\hline \multirow[t]{2}{*}{$M$} & 3526239 & 2835072 & -016 & $101^{\circ}-123 ?$ & & & & & & & \\
\hline & & & +003 & $103^{\circ} 90$ & & & & & & & \\
\hline $\mathrm{K}$ & 3525623 & 2835571 & +006 & $58^{\circ}-83$ & $K$ & & $\left(\begin{array}{ll}0 & 19\end{array}\right)$ & 114 & & & \\
\hline$M$ & 3525142 & 2835957 & -011 & $53^{\circ}-70^{2}$ & & & & & & & \\
\hline$M$ & 3523557 & 2837233 & 000 & $38^{\circ} 16$ & & & & & & & \\
\hline$k$ & 352191 & 283856 & +01 & $118^{\circ} \cdot 134$ & $L$ & & $(0)$ & 131 & & & \\
\hline
\end{tabular}




\begin{tabular}{|c|c|c|c|c|c|c|c|c|c|c|c|}
\hline \multirow{3}{*}{ Reference } & \multirow{3}{*}{$\begin{array}{c}\text { Wave } \\
\text { Length } \\
\lambda(\AA)\end{array}$} & \multicolumn{2}{|c|}{ Frequency } & \multirow{3}{*}{ Transition } & \multirow{3}{*}{ Reference } & \multirow{2}{*}{\multicolumn{5}{|c|}{ Observed Zeeman Pattern }} & \multirow{3}{*}{$\begin{array}{c}\sigma_{230}-\sigma_{232} \\
\left(\mathrm{~cm}^{-1}\right)\end{array}$} \\
\hline & & \multirow{2}{*}{$\begin{array}{c}\sigma_{\mathrm{vac}} \\
\left(\mathrm{cm}^{-1}\right)\end{array}$} & \multirow{2}{*}{$\begin{array}{l}\Delta \sigma_{\text {vac }} \\
\left(\mathrm{cm}^{-1}\right)\end{array}$} & & & & & & & & \\
\hline & & & & & & Type & $p$ & $n$ & $g_{1}$ & $g_{2}$ & \\
\hline $\mathrm{K}$ & 3519690 & 2840350 & +017 & $89^{\circ} 95$ & $M$ & 4 & $\left(\begin{array}{ll}0 & 214\end{array}\right)$ & -0169 & 1328 & 0901 & \\
\hline$M$ & 3518894 & 2840993 & +008 & $52^{\circ} 67$ & $K$ & & $(038)$ & & & & \\
\hline$M$ & 3516827 & 2842662 & 000 & $39^{\circ} 16$ & $M$ & 6 & $(0636)$ & 1395 & 1183 & 1608 & \\
\hline$M$ & 3516357 & 2843042 & -002 & $94^{\circ} 107$ & $K$ & & $\left(\begin{array}{ll}0 & 35\end{array}\right)$ & 103 & & & 078 \\
\hline K & 3515704 & 2843570 & +002 & $97^{\circ} \cdot 151$ & $\mathrm{~K}$ & & $(0)$ & 134 & & & \\
\hline $\mathrm{K}$ & 3515551 & 2843693 & +010 & $100^{\circ} 82$ & & & & & & & \\
\hline$K$ & 351543 & 284379 & 00 & $98^{\circ} 78$ & & & & & & & \\
\hline $\mathrm{K}$ & 3515117 & 2844045 & + 011 & $134^{\circ} \cdot 156^{\circ}$ & & & & & & & \\
\hline$M$ & 3514966 & 2844164 & -005 & $131^{\circ} 171$ & & & & & & & \\
\hline \multirow[t]{2}{*}{$M, K$} & 3514524 & 2844525 & +004 & $81^{\circ} 53$ & $M$ & 6 & $(0330)$ & 1099 & 1166 & 1033 & \\
\hline & & & +015 & $96^{\circ} 110^{\circ}$ & & & & & & & \\
\hline \multirow[t]{2}{*}{ M } & 3512746 & 2845965 & -002 & $96^{\circ} 150$ & $M$ & 4 & $(0097)$ & 0201 & 1168 & 0973 & \\
\hline & & & & & $\mathrm{K}$ & & $(0)$ & $051^{*}$ & & & \\
\hline C & 351163 & 284687 & +01 & $124^{\circ}-136$ & $\mathrm{~L}$ & & $(0)$ & 096 & & & \\
\hline$M$ & 3511553 & 2846932 & 000 & $51^{\circ}-23$ & $M$ & $7 b$ & & 0831 & $(0857)$ & 0874 & \\
\hline$M$ & 3511273 & 2847156 & +006 & $152^{\circ} 158$ & & & & & & & \\
\hline$M$ & 3510540 & 2847753 & -002 & $54^{\circ}-32$ & & & & & & & 068 \\
\hline$M$ & 3509197 & 2848840 & +002 & $102^{\circ} 127$ & & & & & & & \\
\hline$c$ & 350914 & 284889 & 00 & $20^{\circ} .86$ & & & & & & & \\
\hline $\mathrm{K}$ & ו17508 & 2849676 & -004 & $132^{\circ} 114$ & & & & & & & \\
\hline$M$ & 3506854 & 2850746 & +004 & $14^{\circ} 19$ & $M$ & 4 & $(0312)$ & 1279 & 2216 & 1592 & \\
\hline$M$ & 350541 & 285192 & -02 & $55^{\circ} 35^{?}$ & & & & & & & \\
\hline$k$ & 3503610 & 2853386 & +005 & $99^{\circ} 80$ & $L$ & & $(0)$ & 119 & & & \\
\hline$M$ & 3502783 & 2854059 & -004 & $41^{\circ} 52$ & $M$ & 5 & $\left(\begin{array}{lll}0 & 071\end{array}\right)$ & 1657 & 1276 & 1426 & 060 \\
\hline$M$ & 350194 & 285475 & +01 & $52^{\circ} 106$ & & & & & & & \\
\hline$M$ & 3501457 & 2855140 & +004 & $151^{\circ} 172$ & $L$ & & $\left(\begin{array}{ll}0 & 25\end{array}\right)$ & 119 & & & \\
\hline
\end{tabular}

*These data are inconsistent with the classifications listed 
Table 6 (cont inued)

\begin{tabular}{|c|c|c|c|c|c|c|c|c|c|c|c|}
\hline \multirow{3}{*}{ Reference } & \multirow{3}{*}{$\begin{array}{c}\text { Wave } \\
\text { Length } \\
\lambda(\AA)\end{array}$} & \multicolumn{2}{|c|}{ Frequency } & \multirow{3}{*}{ Transition } & \multirow{3}{*}{ Reference } & \multirow{2}{*}{\multicolumn{5}{|c|}{ Observed Zeeman Pattern }} & \multirow{3}{*}{$\begin{array}{c}\sigma_{230}-\sigma_{232} \\
\left(\mathrm{~cm}^{-1}\right)\end{array}$} \\
\hline & & \multirow{2}{*}{$\begin{array}{c}\sigma_{\mathrm{vac}} \\
\left(\mathrm{cm}^{-1}\right) \\
\end{array}$} & \multirow{2}{*}{$\begin{array}{l}\Delta \sigma_{v a c} \\
\left(\mathrm{~cm}^{-1}\right)\end{array}$} & & & & & & & & \\
\hline & & & & & & Type & $p$ & $n$ & $g_{1}$ & $s_{2}$ & \\
\hline \multirow[t]{2}{*}{ C } & 3500.21 & 28561.6 & +0.1 & $98^{\circ}-154$ & & & & & & & \\
\hline & & & -0.1 & $154^{\circ}-160$ & & & & & & & \\
\hline$M$ & 3499.996 & 28563.32 & -0.06 & $40^{\circ} \cdot 16$ & $M$ & 6 & $(0.748)$ & 1.360 & 1.108 & 1.606 & 0.38 \\
\hline \multirow[t]{2}{*}{$M$} & 3498.986 & 28571.56 & +0.05 & $96^{\circ}-111$ & $M$ & 6 & $(0.788)$ & & $(1.173)$ & 0.947 & \\
\hline & & & & & K & & $(0.81)$ & 1.00 & & & \\
\hline K & 3497.96 & 28579.9 & -0.1 & $53^{\circ}-110$ & & & & & & & \\
\hline $\mathrm{K}$ & 3497.459 & 28584.04 & +0.06 & $97^{\circ}-75$ & & & & & & & \\
\hline K & 3497.266 & 28585.61 & 0.00 & $95^{\circ}-109$ & K & & $(0.45)$ & 1.06 & & & \\
\hline $\mathrm{K}$ & 3497.016 & 28587.66 & -0.02 & $147^{\circ}-93$ & $\mathrm{~K}$ & & $(0)$ & 1.10 & & & \\
\hline$M$ & 3496.812 & 28589.30 & +0.01 & $19^{\circ} .39$ & & & & & & & \\
\hline$K$ & 3494.81 & 28605.7 & +0.1 & $13^{\circ}-18$ & & & & & & & \\
\hline$M$ & 3493,526 & 28616.22 & +0.02 & $39^{\circ}-2$ & $M$ & 4 & & 1.150 & 1.185 & $(1.254)$ & \\
\hline$M$ & 3492.683 & 28623.10 & +0.02 & $62^{\circ}-130$ & & & & & & & \\
\hline$M$ & 3491.579 & 28632.14 & +0.07 & $137^{\circ}-161$ & & & & & & & \\
\hline$M$ & 3490.456 & 28641.36 & +0.03 & $3^{\circ}-11$ & $M$ & 5 & $(0.505)$ & 2.060 & 2.565 & 1.555 & \\
\hline $\mathrm{K}$ & 3490.276 & 28642.86 & -0.01 & $152^{\circ} \cdot 179$ & $K$ & & $(0.36)$ & 1.18 & & & \\
\hline$M$ & 3489.832 & 28646.51 & 0.00 & $16^{\circ}-33$ & & & & & & & \\
\hline$M$ & 3487.849 & 28662.79 & -0.08 & $18^{\circ}-38$ & $M$ & 6 & $(0.228)$ & 1.291 & 1.367 & 1.212 & \\
\hline$K$ & 3487.078 & 28669.13 & 0.00 & $96^{\circ}-112$ & & & & & & & \\
\hline \multirow[t]{2}{*}{$M$} & 3486.976 & 28669.97 & +0.01 & $10^{\circ}-17$ & $M$ & 5 & & 1.193 & 0.526 & $(0.968)$ & \\
\hline & & & & & $K$ & & $(0.26)$ & & & & \\
\hline $\mathrm{K}$ & 3486.847 & 28671.03 & +0.06 & $149^{\circ} .95$ & & & & & & & \\
\hline$M$ & 3486.526 & 28673.67 & -0.10 & $151^{\circ}-173$ & $M$ & $6 ?$ & $(0.210)$ & 1.087 & 1.068 & 1.106 & \\
\hline $\mathrm{K}$ & 3485.72 & 28680.3 & 0.0 & $135^{\circ}-158$ & & & & & & & \\
\hline $\mathrm{K}$ & 3485.483 & 28682.25 & -0.06 & $56^{\circ}-82$ & $\mathrm{~K}$ & & $(0.42)$ & & & & 0.83 \\
\hline$M$ & 3485.216 & 28684.45 & -0.06 & $94^{\circ}-148$ & $M$ & 4 & $(0.064)$ & 0.549 & 1.130 & 0.999 & 0.72 \\
\hline$M$ & 3484.086 & 28693.74 & 0.00 & $54^{\circ} \cdot 74$ & $M$ & 6 & $(0.106)$ & 1.110 & 1.089 & 1.131 & \\
\hline$K$ & 3483.263 & 28700.53 & -0.01 & $99^{\circ}-120$ & & & & & & & \\
\hline$M$ & 3482.763 & 28704.65 & 0.00 & $6^{\circ} \cdot 14$ & $M$ & $7 b$ & $(0)$ & 0.590 & $(0.586)$ & 0.57 & \\
\hline$M$ & 3482.552 & 28706.39 & -0.01 & $53^{\circ}-111$ & $M$ & 5 & $(0.177)$ & 1.820 & 0.599 & 0.948 & 0.42 \\
\hline $\mathrm{K}$ & 3481.013 & 28719.08 & -0.06 & $51^{\circ}-102$ & & & & & & & \\
\hline
\end{tabular}




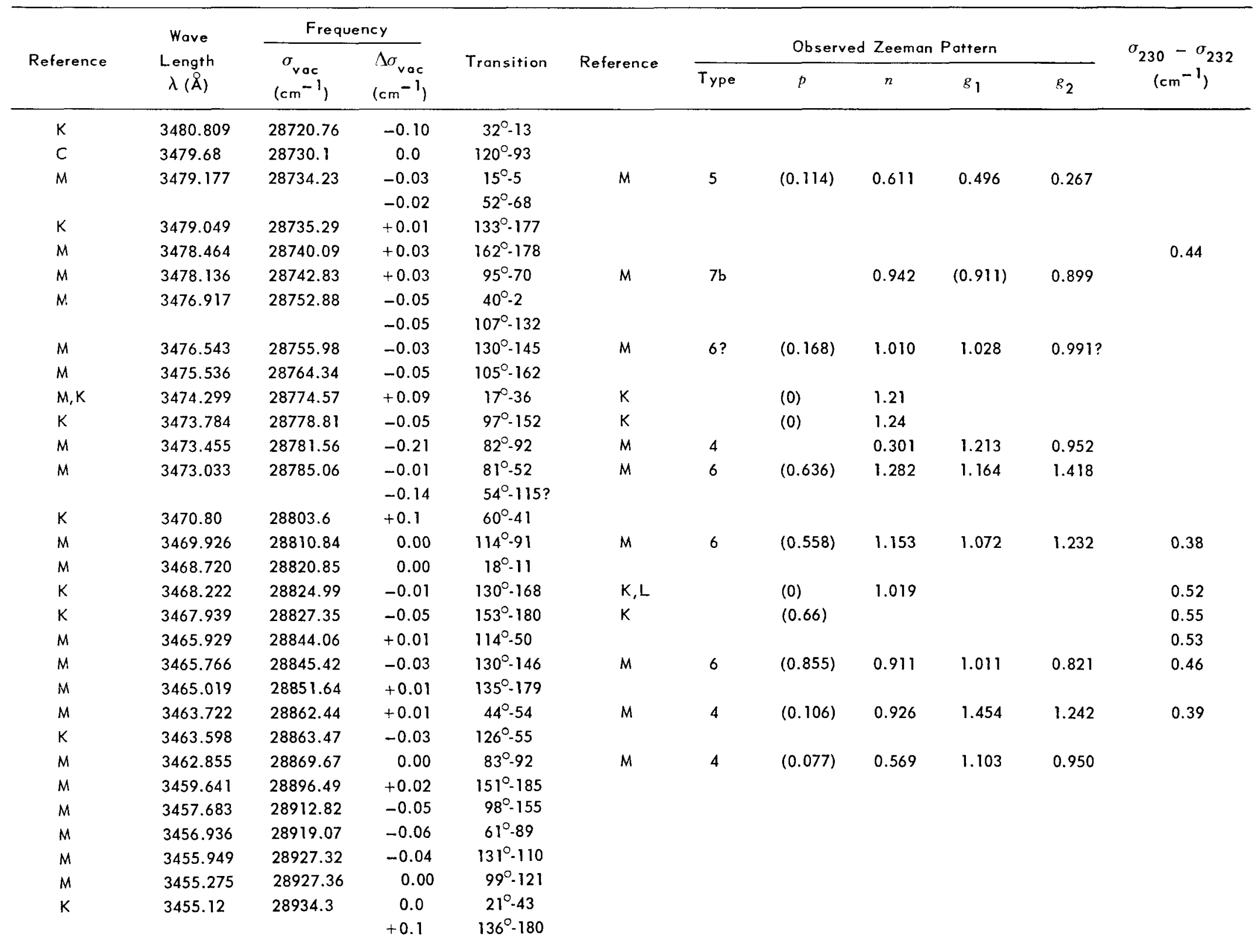


Table 6 (continued)

\begin{tabular}{|c|c|c|c|c|c|c|c|c|c|c|c|}
\hline \multirow{3}{*}{ Reference } & \multirow{3}{*}{$\begin{array}{c}\text { Wave } \\
\text { Length } \\
\lambda(\AA)\end{array}$} & \multicolumn{2}{|c|}{ Frequency } & \multirow{3}{*}{ Transition } & \multirow{3}{*}{ Reference } & \multirow{2}{*}{\multicolumn{5}{|c|}{ Observed Zeeman Pattern }} & \multirow{3}{*}{$\begin{array}{c}\sigma_{230}-\sigma_{232} \\
\left(\mathrm{~cm}^{-1}\right)\end{array}$} \\
\hline & & \multirow{2}{*}{$\begin{array}{c}\sigma_{v o c} \\
\left(\mathrm{~cm}^{-1}\right)\end{array}$} & \multirow{2}{*}{$\begin{array}{l}\Delta \sigma_{\mathrm{vac}} \\
\left(\mathrm{cm}^{-1}\right)\end{array}$} & & & & & & & & \\
\hline & & & & & & Type & $p$ & $n$ & $g_{1}$ & $g_{2}$ & \\
\hline$M$ & 3454.657 & 28938.17 & +0.03 & $46^{\circ}-3$ & & & & & & & \\
\hline K & 3454.205 & 28941.96 & +0.03 & $131^{\circ}-150$ & $\mathrm{~K}$ & & $(0.45)$ & 1.07 & & & 0.41 \\
\hline$M$ & 3453.922 & 28944.30 & -0.01 & $137^{\circ} \cdot 182$ & & & & & & & \\
\hline$M$ & 3453.566 & 28947.32 & -0.02 & $16^{\circ}-10$ & & & & & & & \\
\hline$M$ & 3452.683 & 28954.72 & -0.01 & $9^{\circ} .1$ & $M$ & 6 & $(0.574)$ & 1.542 & 0.969 & 2.116 & \\
\hline K & 3451.702 & 28962.95 & -0.16 & $95^{\circ}-110 ?$ & K & & (0) & $1.31^{\star}$ & & & 0.38 \\
\hline$M$ & 3450.949 & 28969.27 & 0.00 & $86^{\circ}-54$ & $M$ & 6 & $(0.182)$ & 1.240 & 1.162 & $(1.245)$ & 0.44 \\
\hline v & 3450.72 & 28971.2 & -0.1 & $135^{\circ}-122$ & & & & & & & \\
\hline$K$ & 3449.946 & 28977.69 & +0.01 & $95^{\circ}-150$ & & & & & & & \\
\hline$M$ & 3449.648 & 28980.19 & -0.01 & $132^{\circ}-151$ & $M$ & 6 & $(0.723)$ & 1.007 & 0.930 & 1.098 & \\
\hline$M$ & 3447.631 & 28997.15 & -0.03 & $52^{\circ}-26$ & $M$ & 4 & $(0.273)$ & 0.230 & 1.065 & 1.621 & \\
\hline$C$ & 3447.57 & 28997.7 & 0.0 & $102^{\circ}-161$ & & & & & & & \\
\hline$K$ & 3446.990 & 29002.54 & +0.05 & $98^{\circ}-117$ & & & & & & & \\
\hline $\mathrm{K}$ & 3446.541 & 29006.32 & +0.03 & $84^{\circ}-93$ & $K$ & & $(0)$ & 1.19 & & & \\
\hline$M$ & 3445.748 & 29012.99 & +0.11 & $100^{\circ} \cdot 123$ & $M$ & 6 & $(0.567)$ & 0.938 & 0.860 & 1.023 & \\
\hline$M$ & 3445.382 & 29016.07 & +0.05 & $55^{\circ}-117$ & $M$ & $7 b$ & & 1.039 & $(0.982)$ & 0.998 & 0.50 \\
\hline$K$ & 3445.218 & 29017.45 & +0.03 & $119^{\circ}-134$ & $\mathrm{~K}$ & & (0) & 1.210 & & & \\
\hline$K$ & 3445.12 & 29018.3 & +0.1 & $100^{\circ} \cdot 159$ & & & & & & & \\
\hline$M$ & 3444.047 & 29027.32 & -0.11 & $54^{\circ}-75$ & $M$ & $7 b$ & & 1.092 & 1.092 & 1.092 & \\
\hline$M$ & 3443.124 & 29035.10 & -0.12 & $42^{\circ}-53$ & $M$ & 4 & $(0.193)$ & 0.463 & 1.425 & 1.037 & \\
\hline$M$ & 3441.366 & 29049.90 & +0.01 & $15^{\circ}-67$ & $M$ & 5 & $(0.237)$ & 1.639 & 0.470 & 0.938 & 0.47 \\
\hline & & & -0.01 & $134^{\circ}-120$ & & & & & & & \\
\hline$K$ & 3441.036 & 29052.72 & +0.06 & $118^{\circ} \cdot 92$ & $K$ & & $(0.45)$ & 1.03 & & & \\
\hline $\mathrm{K}$ & 3440.91 & 29053.8 & 0.0 & $131^{\circ} \cdot 11$ & & & & & & & \\
\hline & & & +0.2 & $59^{\circ}-85 ?$ & & & & & & & \\
\hline$M$ & 3439.714 & 29063.85 & -0.02 & $147^{\circ}-134$ & $K$ & & $(0.023)$ & 1.144 & 1.141 & 1.147 & \\
\hline$K$ & 3439.229 & 29067.98 & +0.07 & $16^{\circ}-77$ & & & & & & & \\
\hline$M$ & 3438.953 & 29070.32 & +0.01 & $148^{\circ}-135$ & $M$ & 6 & $(0.358)$ & 1.215 & 1.175 & 1.255 & \\
\hline$M$ & 3437.027 & 29086.60 & -0.03 & $94^{\circ}-69$ & $K$ & & $(0)$ & 1.40 & & & 0.79 \\
\hline$M$ & 3436.687 & 29089.48 & -0.04 & $95^{\circ}-111$ & $M$ & 6 & $(0.110)$ & & $(0.911)$ & 0.942 & \\
\hline & & & & & $K$ & & $(0.10)$ & 1.02 & & & \\
\hline
\end{tabular}




\begin{tabular}{|c|c|c|c|c|c|c|c|c|c|c|c|}
\hline \multirow{3}{*}{ Reference } & \multirow{3}{*}{$\begin{array}{c}\text { Wave } \\
\text { Length } \\
\lambda(\AA)\end{array}$} & \multicolumn{2}{|c|}{ Frequency } & \multirow{3}{*}{ Transition } & \multirow{3}{*}{ Reference } & \multirow{2}{*}{\multicolumn{5}{|c|}{ Observed Zeeman Pattern }} & \multirow{3}{*}{$\begin{array}{c}\sigma_{230}-\sigma_{232} \\
\left(\mathrm{~cm}^{-1}\right)\end{array}$} \\
\hline & & \multirow{2}{*}{$\begin{array}{c}\sigma_{v a c} \\
\left(\mathrm{~cm}^{-1}\right)\end{array}$} & \multirow{2}{*}{$\begin{array}{l}\Delta \sigma_{\text {vac }} \\
\left(\mathrm{cm}^{-1}\right)\end{array}$} & & & & & & & & \\
\hline & & & & & & Type & $p$ & $n$ & $g_{1}$ & $g_{2}$ & \\
\hline \multirow[t]{2}{*}{$M$} & 3435.979 & 29095.48 & +0.02 & $72^{\circ}-13$ & $M$ & 5 & $(0.179)$ & 1.536 & 0.999 & 0.643 & 0.70 \\
\hline & & & & & $\mathbf{L}$ & & $(0)$ & $1.09^{\star}$ & & & \\
\hline$M$ & 3434.000 & 29112.24 & 0.00 & $73^{\circ}-14$ & $M$ & 5 & $(0.313)$ & 2.167 & 1.221 & 0.591 & \\
\hline K & 3431.016 & 29137.56 & +0.06 & $99^{\circ} .158$ & $\mathrm{~K}$ & & $(0)$ & 1.15 & & & \\
\hline$M$ & 3430.558 & 29141.45 & +0.13 & $41^{\circ}-17$ & & & & & & & \\
\hline$M$ & 3430.342 & 29143.29 & -0.01 & $53^{\circ}-72$ & & & & & & & \\
\hline \multirow[t]{2}{*}{$M$} & 3429.904 & 29147.01 & +0.04 & $51^{\circ} \cdot 103$ & $M$ & $7 b$ & & 0.793 & $(0.857)$ & 0.839 & 0.78 \\
\hline & & & & & $L$ & & $(0)$ & 0.80 & & & \\
\hline \multirow[t]{2}{*}{$M$} & 3429.392 & 29151.36 & -0.03 & $131^{\circ}-112$ & $M$ & $7 b$ & & 1.090 & 1.090 & 1.090 & \\
\hline & & & -0.08 & $117^{\circ} \cdot 51$ & & & & & & & \\
\hline$M$ & 3427.995 & 29163.21 & -0.02 & $151^{\circ} \cdot 174$ & & & & & & & \\
\hline$M$ & 3427.466 & 29167.74 & +0.05 & $45^{\circ}-54$ & & & & & & & \\
\hline$M$ & 3426.939 & 29172.23 & +0.14 & $53^{\circ}-30 ?$ & & & & & & & \\
\hline $\mathrm{K}$ & 3426.138 & 29179.05 & +0.10 & $19^{\circ} \cdot 40$ & & & & & & & \\
\hline$M$ & 3425.948 & 29180.66 & -0.02 & $83^{\circ}-53$ & $M$ & 6 & $(0.174)$ & 1.068 & 1.103 & 1.033 & \\
\hline$M$ & 3425.190 & 29187.12 & -0.02 & $95^{\circ}-112$ & $M$ & 6 & $(0.612)$ & 0.996 & 0.908 & 1.083 & \\
\hline$K$ & 3423.67 & 29200.1 & -0.1 & $133^{\circ}-118$ & & & & & & & \\
\hline$M$ & 3423.131 & 29204.68 & -0.04 & $55^{\circ}-36$ & $M$ & 4 & $(0.116)$ & 0.633 & 0.982 & 1.215 & \\
\hline$M$ & 3422.657 & 29208.69 & +0.04 & $77^{\circ} \cdot 91$ & & & & & & & \\
\hline$M$ & 3419.176 & 29238.43 & 0.00 & $162^{\circ}-187$ & $\mathrm{~L}$ & & $(0.63)$ & $1.31 ?$ & & & 0.43 \\
\hline \multirow[t]{2}{*}{$K, M$} & 3418.941 & 29240.47 & +0.17 & $81^{\circ}-51$ & & & & & & & \\
\hline & & & -0.18 & $51^{\circ} \cdot 64$ & & & & & & & \\
\hline$M$ & 3418.782 & 29241.80 & -0.06 & $77^{\circ} \cdot 50$ & $\mathrm{~L}$ & & $(0)$ & 0.98 & & & \\
\hline \multirow[t]{2}{*}{$K, M$} & 3417.726 & 29250.87 & +0.04 & $53^{\circ}-31$ & & & & & & & \\
\hline & & & +0.03 & $97^{\circ}-153$ & & & & & & & \\
\hline $\mathrm{C}$ & 3417.69 & 29251.2 & 0.0 & $129^{\circ} \cdot 137$ & & & & & & & \\
\hline$M$ & 3417.12 & 29256.1 & +0.1 & $12^{\circ}-17$ & & & & & & & \\
\hline$K$ & 3416.419 & 29262.05 & -0.01 & $56^{\circ}-38$ & $K$ & & $(0)$ & 1.49 & & & \\
\hline
\end{tabular}

*These data are inconsistent with the classifications listed. 
Table 6 (continued)

\begin{tabular}{|c|c|c|c|c|c|c|c|c|c|c|c|}
\hline \multirow{3}{*}{ Reference } & \multirow{3}{*}{$\begin{array}{c}\text { Wave } \\
\text { Length } \\
\lambda(\AA)\end{array}$} & \multicolumn{2}{|c|}{ Frequency } & \multirow{3}{*}{ Transition } & \multirow{3}{*}{ Reference } & \multirow{2}{*}{\multicolumn{5}{|c|}{ Observed Zeeman Pattern }} & \multirow{3}{*}{$\begin{array}{c}\sigma_{230}-\sigma_{232} \\
\left(\mathrm{~cm}^{-1}\right)\end{array}$} \\
\hline & & $\sigma_{\text {var }}$ & $\Delta \sigma_{\text {vac }}$ & & & & & & & & \\
\hline & & $\left(\mathrm{cm}^{-1}\right)$ & $\left(\mathrm{cm}^{-1}\right)$ & & & Type & $p$ & $n$ & $8_{1}$ & $g_{2}$ & \\
\hline$M$ & 3413.409 & 29287.83 & +0.01 & $93^{\circ} \cdot 145$ & & & & & & & \\
\hline$M$ & 3413.019 & 29291.18 & +0.08 & $149^{\circ}-164$ & & & & & & & \\
\hline$M$ & 3411.789 & 29301.76 & +0.02 & $10^{\circ}-2$ & $M$ & 6 & $(0.369)$ & 0.887 & 0.518 & 1.256 & \\
\hline$M$ & 3409.273 & 29323.38 & 0.00 & $132^{\circ}-152$ & $M$ & 6 & $(0.416)$ & 0.988 & 0.934 & 1.026 & \\
\hline \multirow[t]{2}{*}{$K$} & 3409.104 & 29324.84 & -0.02 & $152^{\circ}-159$ & & & & & & & \\
\hline & & & -0.04 & $103^{\circ}-131$ & & & & & & & \\
\hline$M$ & 3408.648 & 29328.76 & -0.01 & $121^{\circ}-93$ & & & & & & & \\
\hline \multirow[t]{2}{*}{ K } & 3407.631 & 29337.51 & -0.01 & $90^{\circ}-95$ & $M$ & 4 & $(0.121)$ & 0.330 & 1.177 & 0.935 & \\
\hline & & & & & K & & (0) & $0.50^{\star}$ & & & \\
\hline$M$ & 3406.243 & 29349.47 & +0.01 & $15^{\circ}-6$ & $M$ & 4 & $(0.359)$ & 0.131 & 0.490 & 1.208 & \\
\hline$M$ & 3404.654 & 29363.16 & -0.03 & $151^{\circ} \cdot 151$ & $M$ & $7 b$ & & 1.245 & $(1.128)$ & 1.102 & \\
\hline $\mathrm{K}$ & 3404.604 & 29363.60 & -0.07 & $118^{\circ}-53$ & $K, L$ & & $(0)$ & 1.21 & & & \\
\hline$K$ & 3404.201 & 29367.07 & +0.02 & $132^{\circ}-175$ & & & & & & & \\
\hline$M$ & 3403.275 & 29375.06 & -0.02 & $42^{\circ}-52$ & $M$ & $7 b$ & $(0)$ & 1.424 & 1.424 & 1.424 & \\
\hline$K$ & 3403.005 & 29377.39 & +0.13 & $93^{\circ}-146 ?$ & & & & & & & \\
\hline$M$ & 3402.701 & 29379.99 & +0.01 & $115^{\circ}-133$ & $K, L$ & & $(0)$ & 1.33 & & & \\
\hline \multirow[t]{2}{*}{$M$} & 3402.031 & 29385.80 & +0.04 & $81^{\circ}-17$ & $M$ & 5 & $(0.091)$ & & 1.163 & $(0.968)$ & \\
\hline & & & & & $\mathrm{L}$ & & $(0)$ & 1.48 & & & \\
\hline$M$ & 3401.649 & 29389.11 & +0.04 & $38^{\circ}-15$ & $\mathrm{~L}$ & & (0) & 0.82 & & & \\
\hline$M$ & 3401.03 & 29394.5 & -0.1 & $14^{\circ} \cdot 3$ & & & & & & & \\
\hline $\mathrm{K}$ & 3399.328 & 29409.17 & +0.12 & $94^{\circ}-109 ?$ & & & & & & & \\
\hline$k$ & 3399.299 & 29409.42 & -0.11 & $99^{\circ}-82$ & K & & $(0)$ & 1.18 & & & \\
\hline $\mathrm{K}$ & 3397.955 & 29421.07 & +0.07 & $133^{\circ}-156$ & & & & & & & \\
\hline$c$ & 3397.65 & 29423.7 & +0.1 & $98^{\circ}-156$ & & & & & & & \\
\hline$M$ & 3397.108 & 29428.39 & -0.12 & $99^{\circ}-122 ?$ & & & & & & & \\
\hline $\mathrm{K}$ & 3396.615 & 29432.66 & +0.02 & $82^{\circ}-52$ & $K$ & & $(0.49)$ & & & & \\
\hline$K$ & 3396.407 & 29434.46 & 0.00 & $89^{\circ}-55$ & $K$ & & $(0.60)$ & 1.35 & & & \\
\hline $\mathrm{C}$ & 3395.97 & 29438.3 & +0.1 & $65^{\circ}-47$ & & & & & & & \\
\hline$M$ & 1395.381 & 29443.36 & -0.03 & $39^{\circ}-15$ & $M$ & 6 & $(0.564)$ & & 1.176 & $(0.800)$ & \\
\hline \multirow[t]{2}{*}{$M$} & 3395.127 & 29445.56 & -0.01 & $102^{\circ}-128$ & & & & & & & \\
\hline & & & -0.16 & $101^{\circ} \cdot 126 ?$ & & & & & & & \\
\hline
\end{tabular}

*These data are inconsistent with the classifications listed. 
Table 6 (cont inued)

\begin{tabular}{|c|c|c|c|c|c|c|c|c|c|c|c|}
\hline \multirow{3}{*}{ Reference } & \multirow{3}{*}{$\begin{array}{c}\text { Wave } \\
\text { Length } \\
\lambda(\AA)\end{array}$} & \multicolumn{2}{|c|}{ Frequency } & \multirow{3}{*}{ Transition } & \multirow{3}{*}{ Reference } & \multirow{2}{*}{\multicolumn{5}{|c|}{ Observed Zeeman Pattern }} & \multirow{3}{*}{$\begin{array}{c}\sigma_{230}-\sigma_{232} \\
\left(\mathrm{~cm}^{-1}\right)\end{array}$} \\
\hline & & \multirow{2}{*}{$\begin{array}{c}\sigma_{\mathrm{vac}} \\
\left(\mathrm{cm}^{-1}\right)\end{array}$} & \multirow{2}{*}{$\begin{array}{l}\Delta \sigma_{\mathrm{vac}} \\
\left(\mathrm{cm}^{-1}\right)\end{array}$} & & & & & & & & \\
\hline & & & & & & Type & $p$ & $n$ & $g_{1}$ & $g_{2}$ & \\
\hline$M$ & 3393.232 & 29461.97 & -0.09 & $94^{\circ} \cdot 149$ & & & & & & & \\
\hline$M$ & 3392.970 & 29464.28 & -0.14 & $55^{\circ}-37 ?$ & & & & & & & \\
\hline$M$ & 3392.040 & 29472.35 & -0.04 & $112^{\circ}-49$ & $M$ & 5 & $(0.064)$ & 1.505 & 1.195 & 1.071 & \\
\hline$M$ & 3390.781 & 29483.30 & -0.04 & $53^{\circ}-114$ & & & & & & & \\
\hline K & 3390.378 & 29486.80 & -0.07 & $134^{\circ}-158$ & $\mathrm{~K}$ & & $(0)$ & 1.22 & & & \\
\hline$M$ & 3389.645 & 29493.18 & 0.00 & $34^{\circ}-14$ & $M$ & 6 & $(0.412)$ & 0.719 & 0.856 & 0.582 & 0.57 \\
\hline M & 3388.582 & 29502.40 & -0.02 & $131^{\circ}-172$ & $L$ & & $(0)$ & 1.51 & & & \\
\hline$M$ & 3386.504 & 29520.53 & -0.01 & $83^{\circ}-52$ & $M$ & 6 & $(0.780)$ & 1.263 & 1.108 & 1.420 & \\
\hline $\mathrm{K}$ & 3385.84 & 29526.3 & -0.1 & $95^{\circ}-72$ & & & & & & & \\
\hline$M$ & 3385.537 & 29528.96 & -0.02 & $51^{\circ}-24$ & $M$ & $7 b$ & & 0.881 & $\langle 0.857\rangle$ & 0.841 & 0.65 \\
\hline $\mathrm{K}$ & 3385.000 & 29533.65 & +0.03 & $135^{\circ}-159$ & & & & & & & \\
\hline$C$ & 3383.13 & 29550.0 & +0.2 & $54^{\circ}-33 ?$ & $L$ & & $(0)$ & $1.55^{\star}$ & & & \\
\hline$M$ & 3381.375 & 29565.31 & +0.01 & $53^{\circ}-32$ & & & & & & & \\
\hline$M$ & 3380.495 & 29573.01 & +0.03 & $93^{\circ}-67$ & & & & & & & \\
\hline$c$ & 3380.01 & 29577.3 & -0.2 & $58^{\circ}-86 ?$ & & & & & & & \\
\hline$M$ & 3379.674 & 29580.19 & +0.04 & $40^{\circ} \cdot 15$ & & & & & & & \\
\hline$K$ & 3379.30 & 29583.5 & 0.0 & $41^{\circ}-16$ & & & & & & & \\
\hline K & 3379.094 & 29585.26 & +0.04 & $88^{\circ}-19$ & & & & & & & \\
\hline$M$ & 3378.579 & 29589.78 & -0.04 & $11^{\circ} \cdot 16$ & $M$ & 4 & $(0.316)$ & 1.292 & 2.238 & 1.607 & \\
\hline$K$ & 3377.664 & 29597.79 & +0.15 & $100^{\circ}-83 ?$ & & & & & & & \\
\hline$M$ & 3376.848 & 29604.94 & -0.05 & $54^{\circ}-34$ & $M$ & 5 & $(0.190)$ & 1.664 & 1.091 & 0.710 & \\
\hline$K$ & 3374.587 & 29624.78 & -0.05 & $131^{\circ}-173$ & $K, L$ & & $(0)$ & 1.07 & & & 0,45 \\
\hline$M$ & 3373.499 & 29634.30 & +0.10 & $57^{\circ}-125$ & $M$ & 4 & $(0.070)$ & 0.839 & 1.329 & $1.189^{\star}$ & \\
\hline $\mathrm{K}$ & 3371.801 & 29649.25 & +0.06 & $86^{\circ}-94$ & $K, L$ & & $(0)$ & 1.06 & & & \\
\hline$M$ & 3371.49 & 29652.0 & 0.0 & $154^{\circ}-182$ & & & & & & & \\
\hline \multirow[t]{2}{*}{$M$} & 3370.788 & 29658.17 & -0.06 & $133^{\circ}-178 ?$ & & & & & & & \\
\hline & & & -0.07 & $134^{\circ}-179 ?$ & & & & & & & \\
\hline$M$ & 3370.39 & 29661.7 & +0.1 & $95^{\circ}-73$ & & & & & & & \\
\hline
\end{tabular}

*These data are inconsistent with the classifications listed. 
Table 6 (cont inued)

\begin{tabular}{|c|c|c|c|c|c|c|c|c|c|c|c|}
\hline \multirow{3}{*}{ Reference } & \multirow{3}{*}{$\begin{array}{c}\text { Wave } \\
\text { Length } \\
\lambda(\AA)\end{array}$} & \multicolumn{2}{|c|}{ Frequency } & \multirow{3}{*}{ Transition } & \multirow{3}{*}{ Reference } & \multirow{2}{*}{\multicolumn{5}{|c|}{ Observed Zeeman Pattern }} & \multirow{3}{*}{$\begin{array}{c}\sigma_{230}-\sigma_{232} \\
\left(\mathrm{~cm}^{-1}\right)\end{array}$} \\
\hline & & \multirow{2}{*}{$\begin{array}{c}\sigma_{\mathrm{vac}} \\
\left(\mathrm{cm}^{-1}\right)\end{array}$} & \multirow{2}{*}{$\begin{array}{l}\Delta \sigma_{\text {voc }} \\
\left(\mathrm{cm}^{-1}\right)\end{array}$} & & & & & & & & \\
\hline & & & & & & Type & $p$ & $n$ & $g_{1}$ & $g_{2}$ & \\
\hline K & 3366.720 & 29694.00 & +0.04 & $123^{\circ}-54$ & K & & (0) & 1.23 & & & \\
\hline$M$ & 3366.526 & 29695.71 & -0.07 & $123^{\circ}-135$ & $M$ & $7 b$ & (0) & 1.253 & 1.253 & 1.253 & \\
\hline$M$ & 3365.633 & 29703.56 & +0.03 & $118^{\circ}-52$ & $M$ & 4 & & 0.301 & 1.100 & $(1.419)$ & \\
\hline$M$ & 3365.366 & 29705.95 & +0.18 & $2^{\circ}-38 ?$ & & & & & & & \\
\hline K & 3364.84 & 29710.6 & +0.1 & $93^{\circ}-106$ & & & & & & & \\
\hline k & 3364.687 & 29711.94 & +0.01 & $133^{\circ} \cdot 157$ & $M$ & 6 & $(0.687)$ & 1.193 & 1.269 & 1.118 & 0.44 \\
\hline M & 3364.594 & 29712.76 & 0.00 & $100^{\circ}-124$ & $M$ & 6 & $(0.685)$ & & $(0.862)$ & 1.058 & \\
\hline K & 3364.392 & 29714.56 & 0.00 & $98^{\circ}-157$ & & & & & & & \\
\hline$M$ & 3363.737 & 29720.33 & -0.09 & $33^{\circ}-13$ & & & & & & & \\
\hline$K$ & 3363.691 & 29720.73 & 0.00 & $162^{\circ} \cdot 179$ & & & & & & & \\
\hline$M$ & 3363.089 & 29726.05 & -0.21 & $94^{\circ}-71$ & $M$ & $7 b$ & & 1.062 & $(1.132)$ & 1.160 & 0.91 \\
\hline$M$ & 3362.533 & 29730.97 & -0.10 & $147^{\circ} .92$ & & & & & & & \\
\hline$M$ & 3362.196 & 29733.92 & -0.13 & $133^{\circ}-119 ?$ & & & & & & & \\
\hline$C$ & 3361.89 & 29736.7 & 0.0 & $98^{\circ}-119$ & & & & & & & \\
\hline & & & +0.1 & $101^{\circ}-85$ & & & & & & & \\
\hline$M$ & 3361.739 & 29738.00 & -0.06 & $96^{\circ}-115$ & $M$ & 6 & $(0.671)$ & & 1.158 & 0.966 & 0.47 \\
\hline$M$ & 3361.68 & 29738.5 & +0.1 & $54^{\circ}-116$ & $\mathrm{~L}$ & & $(0)$ & 1.12 & & & \\
\hline$K$ & 3360.369 & 29750.12 & +0.08 & $151^{\circ}-175$ & $K, L$ & & $(0)$ & 1.11 & & & \\
\hline & & & -0.09 & $55^{\circ} \cdot 119$ & & & & & & & \\
\hline$M$ & 3360.161 & 29751.96 & -0.05 & $16^{\circ}-79$ & $M$ & 5 & $(0.140)$ & 1.510 & 0.835 & 1.107 & \\
\hline$M$ & 3359.070 & 29761.59 & -0.03 & $100^{\circ}-84$ & & & & & & & \\
\hline$M$ & 3358.606 & 29765.73 & -0.08 & $7^{0} \cdot 14$ & $M$ & 5 & $(0.121)$ & 0.706 & 0.343 & 0.585 & \\
\hline$K$ & 3357.404 & 29776.39 & +0.03 & $153^{\circ}-160$ & & & & & & & \\
\hline$M$ & 3357.232 & 29777.89 & +0.01 & $134^{\circ}-122$ & & & & & & & \\
\hline$M$ & 3357.054 & 29779.50 & +0.10 & $11^{\circ}-2 ?$ & & & & & & & \\
\hline$M$ & 3356.829 & 29781.46 & -0.03 & $53^{\circ}-74$ & & & & & & & \\
\hline$K$ & 3356.25 & 29786.6 & +0.1 & $94^{\circ} \cdot 110$ & & & & & & & \\
\hline$M$ & 3355.245 & 29795.52 & +0.16 & $132^{\circ} \cdot 153 ?$ & & & & & & & \\
\hline$c$ & 3354.81 & 29799.4 & -0.1 & $102^{\circ} \cdot 129$ & & & & & & & \\
\hline$K$ & 3354.623 & 29801.07 & -0.05 & $94^{\circ} \cdot 150$ & K & & $(0)$ & 0.54 & & & \\
\hline$K$ & 3354.180 & 29805.01 & +0.02 & $121^{\circ} \cdot 134$ & $K, L$ & & $(0)$ & 1.03 & & & \\
\hline$M$ & 3353.96 & 29807.0 & 0.0 & $97^{\circ} .78$ & $M$ & $7 b$ & & 0.977 & 0.977 & 0.977 & 0.41 \\
\hline$M$ & 3353.16 & 29814.1 & -0.1 & $20^{\circ}-42$ & & & & & & & \\
\hline
\end{tabular}


Table 6 (continued)

\begin{tabular}{|c|c|c|c|c|c|c|c|c|c|c|c|}
\hline \multirow{3}{*}{ Reference } & \multirow{3}{*}{$\begin{array}{c}\text { Wave } \\
\text { Length } \\
\lambda(\AA)\end{array}$} & \multicolumn{2}{|c|}{ Frequency } & \multirow{3}{*}{ Transition } & \multirow{3}{*}{ Reference } & \multirow{2}{*}{\multicolumn{5}{|c|}{ Observed Zeeman Pattern }} & \multirow{3}{*}{$\begin{array}{c}\sigma_{230}-\sigma_{232} \\
\left(\mathrm{~cm}^{-1}\right)\end{array}$} \\
\hline & & \multirow{2}{*}{$\begin{array}{c}\sigma_{v a c} \\
\left(\mathrm{~cm}^{-1}\right)\end{array}$} & \multirow{2}{*}{$\begin{array}{c}\Delta \sigma_{\text {vac }} \\
\left(\mathrm{cm}^{-1}\right)\end{array}$} & & & & & & & & \\
\hline & & & & & & Type & $p$ & $n$ & $g_{1}$ & $g_{2}$ & \\
\hline$M$ & 3351.599 & 29827.96 & 0.00 & $81^{\circ}-16$ & & & & & & & \\
\hline$M$ & 3351.230 & 29831.25 & +0.04 & $34^{\circ}-49$ & $M$ & 5 & $(0.107)$ & 1.389 & 0.866 & 1.076 & 0.48 \\
\hline$M$ & 3350.311 & 29839.39 & -0.04 & $132^{\circ}-116$ & & & & & & & \\
\hline$M$ & 3349.884 & 29839.43 & 0.00 & $56^{\circ}-83$ & & & & & & & \\
\hline \multirow[t]{2}{*}{$M$} & 3347.576 & 29863.80 & +0.05 & $2^{\circ}-11$ & & & & & & & \\
\hline & & & -0.03 & $60^{\circ}-90$ & & & & & & & \\
\hline \multirow[t]{2}{*}{ K } & 3347.28 & 29866.5 & 0.0 & $95^{\circ}-114$ & & & & & & & \\
\hline & & & 0.0 & $98^{\circ} \cdot 80$ & & & & & & & \\
\hline$M$ & 3346.559 & 29872.88 & -0.07 & $53^{\circ}-115$ & $M$ & 5 & $(0.185)$ & 1.929 & 0.608 & 0.985 & 0.45 \\
\hline$M$ & 3345.38 & 29883.4 & 0.0 & $136^{\circ}-160$ & & & & & & & \\
\hline \multirow[t]{2}{*}{$M$} & 3344.882 & 29887.86 & +0.10 & $12^{\circ} \cdot 2$ & & & & & & & \\
\hline & & & -0.09 & $61^{\circ}-130$ & $L$ & & $(0)$ & $1.44 ?$ & & & \\
\hline$K$ & 3344.31 & 29893.0 & 0.0 & $44^{\circ}-18$ & & & & & & & \\
\hline$M$ & 3343.815 & 29897.40 & +0.02 & $93^{\circ} \cdot 68$ & & & & & & & \\
\hline$M$ & 3343.271 & 29902.23 & 0.00 & $127^{\circ}-55$ & $M$ & 4 & $(0.165)$ & 0.667 & 1.241 & $(1.571)$ & \\
\hline$K$ & 3342.07 & 29913.0 & 0.0 & $94^{\circ}-111$ & & & & & & & \\
\hline$c$ & 3338.84 & 29941.9 & -0.1 & $101^{\circ}-127$ & & & & & & & \\
\hline \multirow[t]{2}{*}{$M$} & 3338.400 & 29945.89 & +0.01 & $52^{\circ}-108$ & $M$ & 4 & $(0.092)$ & & $(1.061)$ & 0.882 & \\
\hline & & & & & $K$ & & (0) & 0.51 & & & \\
\hline$M$ & 3337.869 & 29950.64 & +0.01 & $74^{\circ} \cdot 14$ & $M$ & 5 & $(0.266)$ & 1.908 & 1.112 & 0.580 & \\
\hline$k$ & 3337.16 & 29957.0 & -0.1 & $93^{\circ}-147$ & & & & & & & \\
\hline \multirow[t]{2}{*}{$k$} & 3335.062 & 29975.86 & -0.09 & $42^{\circ} \cdot 17$ & $K$ & & $(0.69)$ & 1.10 & & & \\
\hline & & & +0.09 & $83^{\circ} \cdot 51$ & & & & & & & \\
\hline$M$ & 3334.605 & 29979.97 & -0.18 & $145^{\circ}-133$ & $M$ & 6 & $(0.730)$ & 1.228 & 1.148 & 1.311 & 0.45 \\
\hline$K$ & 3334.055 & 29984.91 & +0.07 & $89^{\circ}-19$ & $\mathrm{~K}$ & & $(0)$ & 1.20 & & & \\
\hline$K$ & 3333.384 & 29990.95 & +0.09 & $99^{\circ} \cdot 159$ & & & & & & & \\
\hline$M$ & 3332.395 & 29999.85 & +0.03 & $86^{\circ}-18$ & $M$ & 5 & $(0.102)$ & 1.469 & 1.173 & 0.977 & \\
\hline$M$ & 3332.07 & 30002.8 & 0.0 & $132^{\circ}-176$ & & & & & & & \\
\hline \multirow[t]{2}{*}{$\mathrm{K}$} & 3328.67 & 30033.4 & +0.2 & $98^{\circ}-120 ?$ & & & & & & & \\
\hline & & & +0.1 & $82^{\circ} \cdot 17$ & & & & & & & \\
\hline
\end{tabular}


Table 6 (continued)

\begin{tabular}{|c|c|c|c|c|c|c|c|c|c|c|c|}
\hline \multirow{3}{*}{ Reference } & \multirow{3}{*}{$\begin{array}{c}\text { Wave } \\
\text { Length } \\
\lambda(\AA)\end{array}$} & \multicolumn{2}{|c|}{ Frequency } & \multirow{3}{*}{ Transition } & \multirow{3}{*}{ Reference } & \multirow{2}{*}{\multicolumn{5}{|c|}{ Observed Zeeman Pattern }} & \multirow{3}{*}{$\begin{array}{c}\sigma_{230}-\sigma_{232} \\
\left(\mathrm{~cm}^{-1}\right)\end{array}$} \\
\hline & & \multirow{2}{*}{$\begin{array}{c}\sigma_{\text {vac }} \\
\left(\mathrm{cm}^{-1}\right)\end{array}$} & \multirow{2}{*}{$\begin{array}{c}\Delta \sigma_{\mathrm{vac}} \\
\left(\mathrm{cm}^{-1}\right)\end{array}$} & & & & & & & & \\
\hline & & & & & & Type & $p$ & $n$ & $g_{1}$ & $g_{2}$ & \\
\hline$M$ & 3328.25 & 30037.2 & +0.1 & $46^{\circ}-54$ & $M$ & 4 & $(0.159)$ & 0.763 & 1.556 & 1.240 & \\
\hline$M$ & 3327.194 & 30046.74 & +0.01 & $55^{\circ}-120$ & $M$ & 5 & $(0)$ & 1.300 & $(0.982)$ & 1.072 & \\
\hline$M$ & 3326.468 & 30053.30 & -0.04 & $51^{\circ} .66$ & $M$ & 6 & $(0.358)$ & 0.930 & 0.860 & 1.000 & \\
\hline M & 3325.132 & 30065.38 & -0.08 & $78^{\circ}-91$ & $M$ & 5 & & 1.400 & 1.166 & $(1.232)$ & \\
\hline$M$ & 3324.754 & 30068.79 & -0.04 & $35^{\circ}-14$ & M & 6 & $(0.457)$ & 0.732 & 0.883 & 0.579 & 0.39 \\
\hline M & 3321.453 & 30098.68 & +0.01 & $78^{\circ}-50$ & $M$ & $7 b$ & $(0)$ & 1.152 & 1.152 & 1.152 & \\
\hline$M$ & 3320.302 & 30109.11 & +0.01 & $130^{\circ} \cdot 107$ & $M$ & $7 b$ & & 1.001 & $(1.018)$ & 1.023 & \\
\hline$M$ & 3319.63 & 30115.2 & 0.0 & $53^{\circ}-75$ & & & & & & & \\
\hline$M$ & 3318.981 & 30121.09 & 0.00 & $15^{\circ}-7$ & $M$ & 4 & $(0.123)$ & 0.361 & 0.489 & 0.735 & \\
\hline$M$ & 3318.21 & 30128.1 & 0.0 & $59^{\circ}-41$ & & & & & & & \\
\hline$K$ & 3318.12 & 30128.9 & 0.0 & $10^{\circ}-15$ & & & & & & & \\
\hline$M$ & 3317.571 & 30133.89 & -0.08 & $52^{\circ} \cdot 69$ & $M$ & 76 & & 1.053 & 1.053 & 1.053 & \\
\hline$M$ & 3316.23 & 30146.1 & -0.1 & $38^{\circ}-1$ & $M$ & 4 & $(0.643)$ & 0.187 & 0.830 & 2.116 & \\
\hline$K$ & 3315.83 & 30149.7 & 0.0 & $84^{\circ} .92$ & & & & & & & \\
\hline$c$ & 3315.55 & 30152.3 & +0.1 & $136^{\circ}-127$ & & & & & & & \\
\hline$K$ & 3314.831 & 30158.77 & +0.01 & $118^{\circ}-51$ & $M$ & 5 & $(0.052)$ & 1.364 & 1.104 & 1.000 & \\
\hline C & 3314.01 & 30166.3 & +0.1 & $62^{\circ}-46$ & & & & & & & \\
\hline v & 3313.02 & 30175.3 & +0.1 & $96^{\circ} \cdot 152$ & & & & & & & \\
\hline \multirow[t]{2}{*}{$M$} & 3310.51 & 30198.2 & -0.2 & $2^{\circ}-39 ?$ & & & & & & & \\
\hline & & & -0.1 & $45^{\circ}-18$ & & & & & & & \\
\hline \multirow[t]{2}{*}{$M$} & 3310.249 & 30200.55 & +0.04 & $39^{\circ}-1$ & $M$ & 4 & $(0.454)$ & 0.714 & 1.168 & 2.076 & \\
\hline & & & & & $L$ & & (0) & $1.07^{\star}$ & & & \\
\hline$M$ & 3309.137 & 30210.69 & 0.00 & $52^{\circ}-27$ & $M$ & 5 & & 1.123 & $(1.061)$ & 1.018 & \\
\hline$M$ & 3308.071 & 30220.40 & +0.08 & $131^{\circ} \cdot 115$ & & & & & & & \\
\hline $\mathrm{K}$ & 3306.365 & 30236.02 & -0.29 & $51^{\circ}-105 ?$ & $K$ & & $(0)$ & 1.01 & & & \\
\hline$M$ & 3304.174 & 30256.07 & 0.00 & $95^{\circ}-115$ & $M$ & 6 & $(0.200)$ & 0.900 & $(0.911)$ & 0.968 & \\
\hline$M$ & 3303.487 & 30262.33 & -0.03 & $56^{\circ} \cdot 125$ & $M$ & 4 & $(0.134)$ & 0.439 & 1.339 & 1.081 & \\
\hline
\end{tabular}

* These data are inconsistent with the classifications listed. 


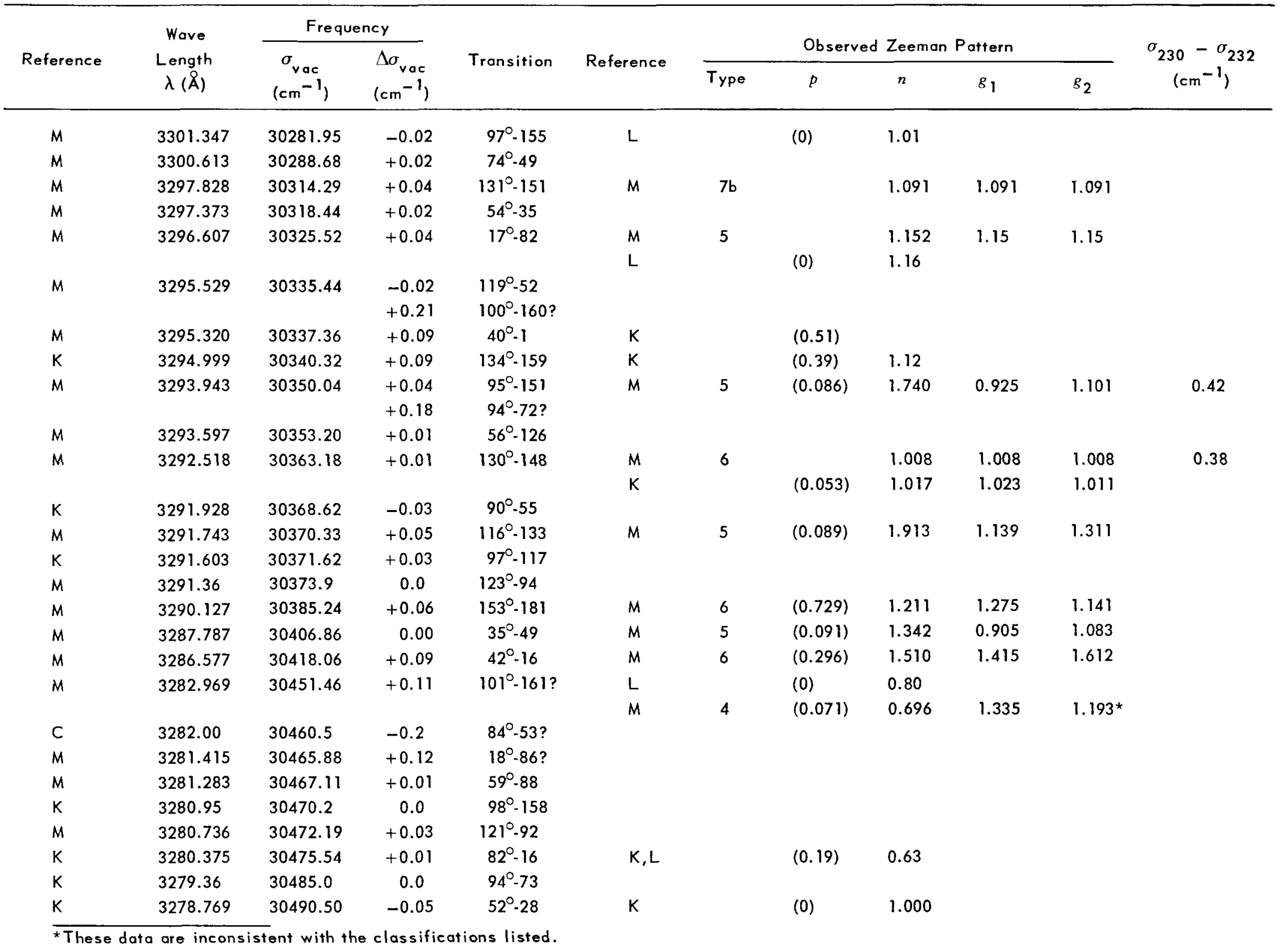


Table 6 (continued)

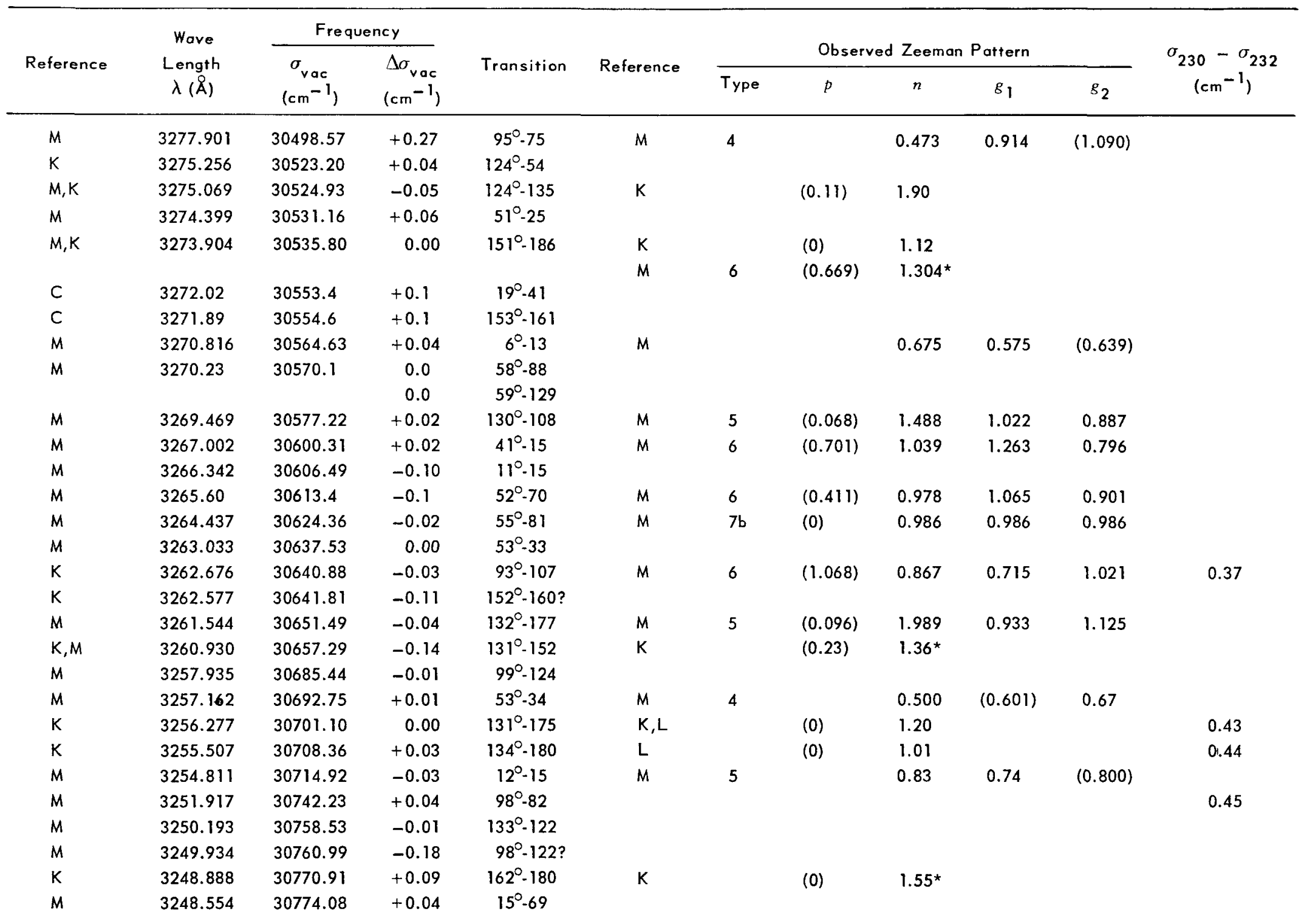




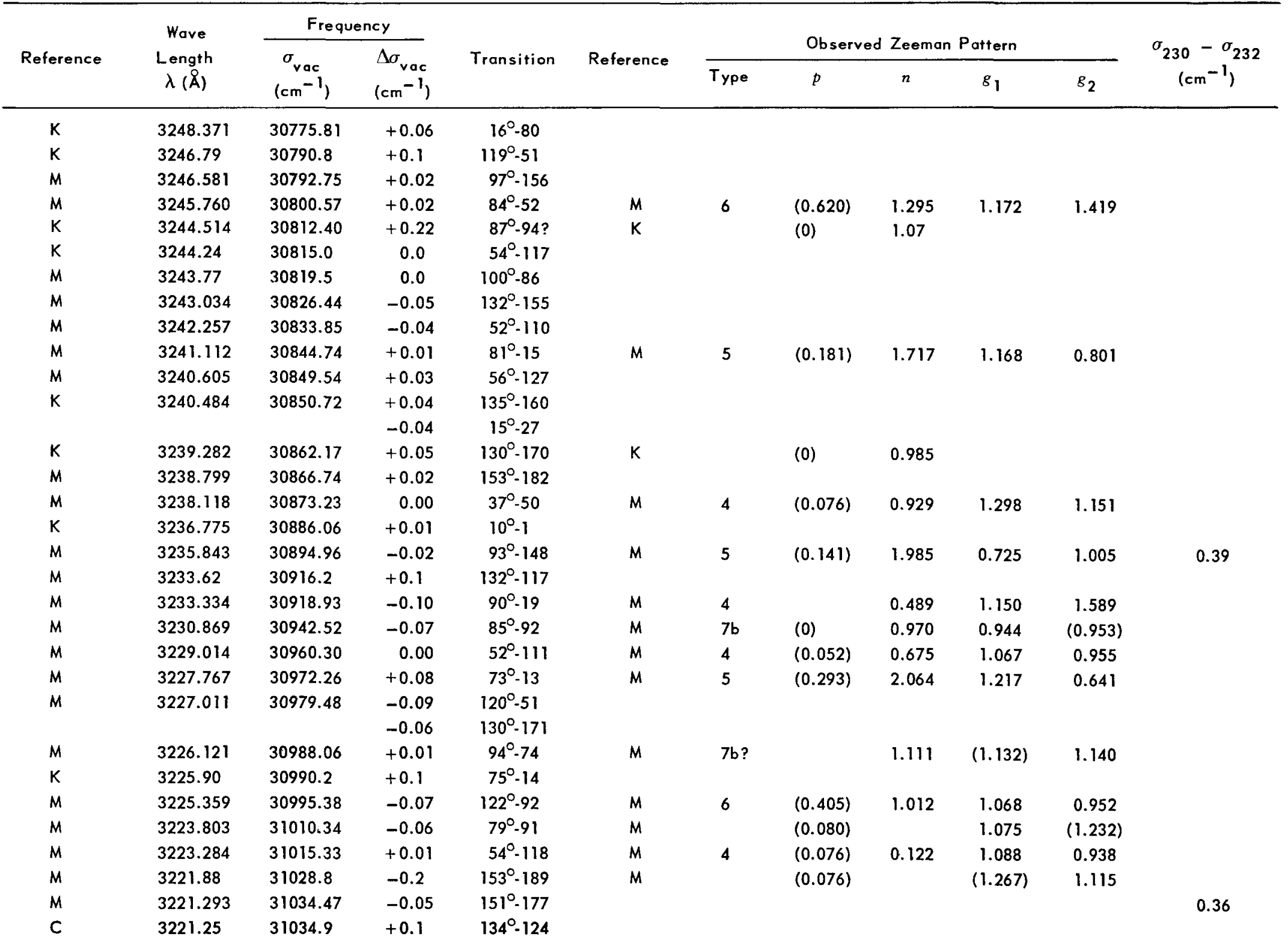


Table 6 (continued)

\begin{tabular}{|c|c|c|c|c|c|c|c|c|c|c|c|}
\hline \multirow{3}{*}{ Reference } & \multirow{3}{*}{$\begin{array}{c}\text { Wave } \\
\text { Length } \\
\lambda(\AA)\end{array}$} & \multicolumn{2}{|c|}{ Frequency } & \multirow{3}{*}{ Transition } & \multirow{3}{*}{ Reference } & \multirow{2}{*}{\multicolumn{5}{|c|}{ Observed Zeeman Pattern }} & \multirow{3}{*}{$\begin{array}{c}\sigma_{230}-\sigma_{232} \\
\left(\mathrm{~cm}^{-1}\right)\end{array}$} \\
\hline & & \multirow{2}{*}{$\begin{array}{c}\sigma_{\mathrm{vac}} \\
\left(\mathrm{cm}^{-1}\right)\end{array}$} & \multirow{2}{*}{$\begin{array}{l}\Delta \sigma_{\text {vac }} \\
\left(\mathrm{cm}^{-1}\right)\end{array}$} & & & & & & & & \\
\hline & & & & & & Type & $p$ & $n$ & $g_{1}$ & $g_{2}$ & \\
\hline$M$ & 3220.348 & 31043.61 & 0.00 & $79^{\circ}-50$ & $M$ & 6 & $(0.191)$ & 1.140 & 1.087 & 1.163 & 0.72 \\
\hline$M$ & 3218.864 & 31057.92 & 0.00 & $52^{\circ}-112$ & $M$ & 5 & & 1.130 & $(1.061)$ & 1.082 & \\
\hline k & 3218.76 & 31058.9 & 0.0 & $53^{\circ}-77$ & & & & & & & \\
\hline \multirow[t]{2}{*}{$M$} & 3218.317 & 31063.19 & -0.01 & $17^{\circ} \cdot 11$ & & & & & & & \\
\hline & & & +0.13 & $150^{\circ}-136 ?$ & & & & & & & \\
\hline$M$ & 3217.856 & 31067.65 & -0.03 & $46^{\circ} \cdot 18$ & & & & & & & \\
\hline$M$ & 3217.733 & 31068.81 & -0.07 & $161^{\circ}-134$ & & & & & & & \\
\hline$M$ & 3217.457 & 31071.50 & +0.02 & $43^{\circ}-53$ & $M$ & 4 & $(0.104)$ & 0.704 & 1.232 & 1.020 & \\
\hline $\mathrm{V}$ & 3216.63 & 31079.5 & 0.0 & $94^{\circ}-115$ & & & & & & & \\
\hline K & 3215.772 & 31087.78 & +0.07 & $130^{\circ} \cdot 109$ & $K$ & & (0) & 1.00 & & & \\
\hline$k$ & 3213.573 & 31109.05 & +0.05 & $93^{\circ}-108$ & $M$ & 6 & $(0.557)$ & 0.795 & 0.714 & 0.874 & \\
\hline$M$ & 3212.811 & 31116.43 & +0.04 & $132^{\circ}-118$ & $M$ & $7 b ?$ & & 0.907 & $(0.931)$ & 0.938 & \\
\hline$K$ & 3212.130 & 31123.03 & 0.00 & $121^{\circ}-52$ & & & & & & & \\
\hline V & 3211.45 & 31129.6 & +0.2 & $131^{\circ}-153 ?$ & & & & & & & \\
\hline$K$ & 3211.34 & 31130.7 & +0.1 & $15^{\circ} \cdot 28$ & & & & & & & \\
\hline C & 3210.32 & 31140.6 & -0.1 & $130^{\circ}-149$ & $\mathbf{L}$ & & $(0.26)$ & 1.07 & & & \\
\hline$M$ & 3209.33 & 31150.2 & 0.0 & $101^{\circ}-88$ & $M$ & 4 or 5 & $(0.092)$ & & 1.296 & $(1.112)$ & \\
\hline$M$ & 3208.025 & 31162.84 & +0.03 & $87^{\circ} \cdot 18$ & $M$ & 5 & $(0.112)$ & 1.534 & 1.213 & 0.993 & \\
\hline$K$ & 3207.785 & 31165.19 & +0.03 & $95^{\circ}-153$ & & & & & & & \\
\hline \multirow[t]{2}{*}{$M$} & 3206.935 & 31173.45 & +0.01 & $94^{\circ}-151$ & $M$ & $7 b$ & $(0)$ & 1.052 & $(1.132)$ & $1.114 ?$ & \\
\hline & & & -0.03 & $131^{\circ} \cdot 116$ & $M$ & $7 b$ & $(0)$ & 1.052 & $(1.086)$ & 1.097 & \\
\hline \multirow[t]{2}{*}{$M, K$} & 3203.874 & 31203.22 & +0.14 & $124^{\circ}-94$ & $M$ & 6 & $(0.296)$ & 1.084 & 1.042 & 1.126 & \\
\hline & & & -0.07 & $96^{\circ} .78$ & & & & & & & \\
\hline \multirow[t]{2}{*}{$K, M$} & 3203.246 & 31209.34 & -0.14 & $151^{\circ}-155$ & & & & & & & \\
\hline & & & +0.11 & $95^{\circ}-116$ & & & & & & & \\
\hline$M$ & 3201.225 & 31229.02 & +0.02 & $60^{\circ}-44$ & $M$ & & $(0.283)$ & & 1.488 & $(0.922)$ & \\
\hline$M$ & 3200.557 & 31235.56 & -0.01 & $97^{\circ} \cdot 80$ & $M$ & 4 & $(0.073)$ & 0.608 & 0.969 & 1.111 & \\
\hline$K$ & 3198.973 & 31251.03 & -0.04 & $51^{\circ}-67$ & $\mathrm{~K}$ & & $(0.18)$ & 0.94 & & & 0.94 \\
\hline \multirow[t]{2}{*}{$M$} & 3198.702 & 31253.65 & +0.05 & $85^{\circ}-53$ & $\mathrm{~K}$ & & $(0.20)$ & 1.04 & & & \\
\hline & & & +0.03 & $15^{\circ} .70$ & & & & & & & \\
\hline$K$ & 3198.480 & 31255.85 & +0.07 & $84^{\circ}-51$ & $\mathrm{~K}$ & & $(0.46)$ & & & & \\
\hline$K$ & 3194.25 & 31297.2 & +0.1 & $93^{\circ} \cdot 69$ & & & & & & & \\
\hline$M$ & 3193.311 & 31306.44 & -0.02 & $122^{\circ}-53$ & $M$ & 5 & $(0)$ & 1.136 & 1.062 & $(1.034)$ & \\
\hline
\end{tabular}




\begin{tabular}{|c|c|c|c|c|c|c|c|c|c|c|c|}
\hline \multirow{3}{*}{ Reference } & \multirow{3}{*}{$\begin{array}{c}\text { Wave } \\
\text { Length } \\
\lambda(\AA)\end{array}$} & \multicolumn{2}{|c|}{ Frequency } & \multirow{3}{*}{ Transition } & \multirow{3}{*}{ Reference } & \multirow{2}{*}{\multicolumn{5}{|c|}{ Observed Zeeman Pattern }} & \multirow{3}{*}{$\begin{array}{c}\sigma_{230}-\sigma_{232} \\
\left(\mathrm{~cm}^{-1}\right)\end{array}$} \\
\hline & & $\sigma_{v}$ & $\Delta \sigma_{\text {yar }}$ & & & & & & & & \\
\hline & & $\left(\mathrm{cm}^{-1}\right)$ & $\left(\mathrm{cm}^{-1}\right)$ & & & Type & $p$ & $n$ & $g_{1}$ & $g_{2}$ & \\
\hline$M$ & 3193.17 & 31307.8 & -0.1 & $99^{\circ}-160$ & & & & & & & \\
\hline$M$ & 3192.098 & 31318.31 & +0.08 & $98^{\circ}-123$ & & & & & & & \\
\hline$M$ & 3191.835 & 21320.89 & 0.00 & $133^{\circ}-159$ & & & & & & & \\
\hline$K$ & 3191.219 & 31326.96 & +0.06 & $96^{\circ}-154$ & $K$ & & $(0)$ & 0.95 & & & \\
\hline$M$ & 3191.095 & 31328.18 & +0.05 & $75^{\circ}-49$ & $\mathrm{~K}$ & & $(0.81)$ & $0.87^{\star}$ & & & \\
\hline$M$ & 3190.73 & 31331.8 & 0.0 & $55^{\circ}-123$ & & & & & & & \\
\hline$M$ & 3190.34 & 31335.6 & +0.1 & $55^{\circ}-38$ & & & & & & & \\
\hline \multirow[t]{2}{*}{$\mathrm{K}$} & 3190.169 & 31337.27 & +0.02 & $132^{\circ}-156$ & $M$ & 6 & $(0.754)$ & & $(0.931)$ & 1.097 & \\
\hline & & & & & $M$ & 6 & $(0.754)$ & 0.933 & 0.849 & $1.017^{\star}$ & \\
\hline$M$ & 3190.074 & 31338.20 & +0.02 & $53^{\circ}-78$ & $M$ & 6 & $(1.000)$ & & $(0.601)$ & 1.001 & \\
\hline$K$ & 3189.04 & 31348.4 & 0.0 & $47^{\circ}-3$ & & & & & & & \\
\hline$M$ & 3188.281 & 31355.84 & 0.00 & $36^{\circ}-14$ & $M$ & 6 & $(0.677)$ & 0.814 & 1.039 & 0.588 & \\
\hline$M$ & 3188.188 & 1356.735 & -0.18 & $60^{\circ}-45 ?$ & & & & & & & \\
\hline $\mathrm{K}$ & 3188.116 & 31357.45 & +0.04 & $41^{\circ}-1$ & & & & & & & \\
\hline$K$ & 3187.46 & $31363.9 ?$ & +0.2 & $11^{\circ} \cdot 1 ?$ & & & & & & & \\
\hline $\mathrm{K}$ & 3187.402 & 31364.47 & +0.04 & $86^{\circ}-93$ & $K$ & & $(0)$ & 1.17 & & & \\
\hline$M$ & 3184.952 & 31388.60 & 0.00 & $51^{\circ}-106$ & $M$ & 5 & $(0.062)$ & 1.311 & 0.869 & 0.996 & 0.71 \\
\hline$M$ & 3183.794 & 31400.02 & 0.00 & $126^{\circ}-54$ & $M$ & $7 b$ & $(0)$ & 1.230 & 1.230 & 1.230 & \\
\hline $\mathrm{K}$ & 3183.565 & 31402.27 & -0.03 & $97^{\circ}-120$ & $\mathrm{~K}$ & & $(0.24)$ & 1.11 & & & \\
\hline$M$ & 3182.648 & 31411.32 & -0.02 & $43^{\circ}-52$ & $M$ & 5 & $(0.085)$ & 1.667 & 1.254 & 1.423 & \\
\hline$M$ & 3182.403 & 31413.74 & +0.07 & $15^{\circ}-71$ & & & & & & & \\
\hline$M$ & 3181.76 & 31420.1 & 0.0 & $152^{\circ}-161$ & & & & & & & \\
\hline$M$ & 3181.189 & 31425.73 & -0.26 & $52^{\circ}-30$ & $M$ & 5 & $(0.063)$ & 1.257 & 1.071 & 0.947 & \\
\hline$M$ & 3180.199 & 31435.51 & +0.01 & $114^{\circ}-49$ & $M$ & $7 b$ & $(0)$ & 1.074 & 1.074 & 1.074 & 0.39 \\
\hline$K$ & 3179.046 & 31446.88 & -0.08 & $115^{\circ}-91$ & $L$ & & $(0.24)$ & 0.82 & & & 0.57 \\
\hline$K$ & 3178.787 & 31449.47 & -0.01 & $101^{\circ}-89$ & $\mathrm{~K}$ & & $(0)$ & 1.67 & & & \\
\hline$M$ & 3177.874 & 31458.51 & +0.10 & $18^{\circ} \cdot 88$ & $M$ & 4 & $(0.123)$ & $0: 720$ & 1.35 & 1.10 & \\
\hline$K$ & 3177.37 & 31463.5 & +0.2 & $136^{\circ}-129 ?$ & & & & & & & \\
\hline
\end{tabular}

*These data are inconsistent with the elassifications listed. 
Table 6 (continued)

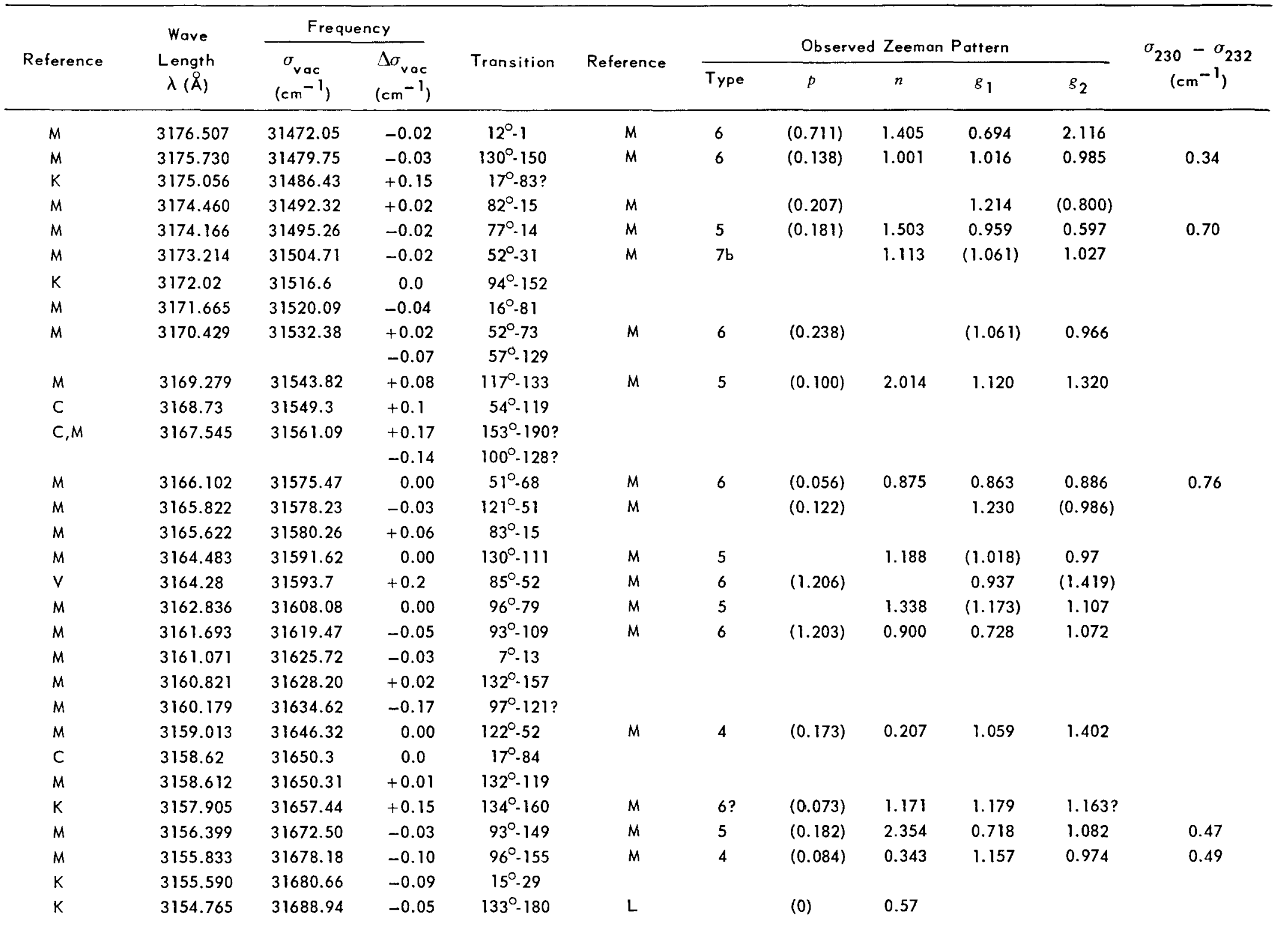




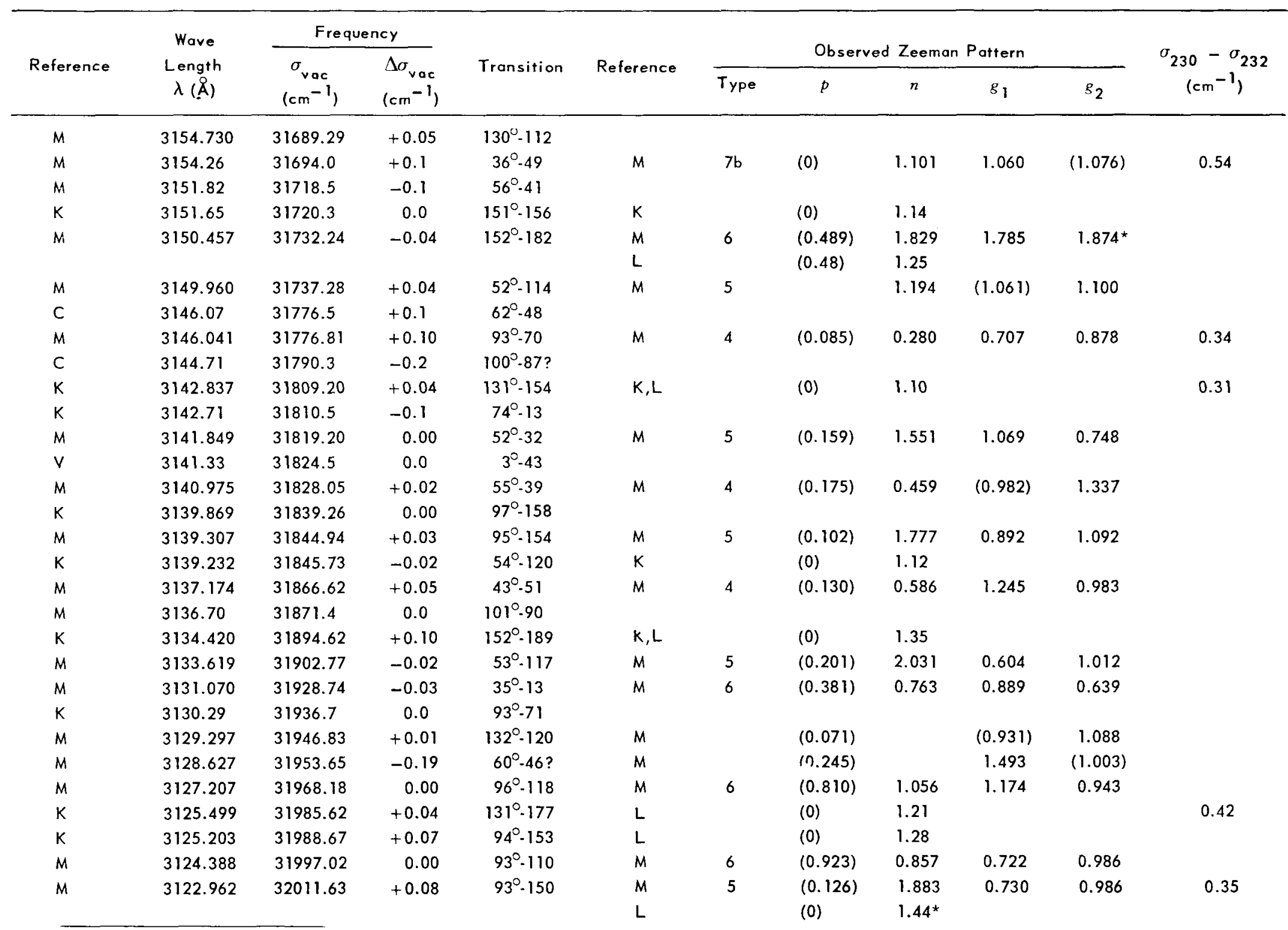

*These data are inconsistent with the classifications listed. 
Table 6 (cont inued)

\begin{tabular}{|c|c|c|c|c|c|c|c|c|c|c|c|}
\hline \multirow{3}{*}{ Reference } & \multirow{3}{*}{$\begin{array}{c}\text { Wave } \\
\text { Length } \\
\lambda(\AA)\end{array}$} & \multicolumn{2}{|c|}{ Frequency } & \multirow{3}{*}{ Transition } & \multirow{3}{*}{ Reference } & \multirow{2}{*}{\multicolumn{5}{|c|}{ Observed Zeeman Pattern }} & \multirow{3}{*}{$\begin{array}{c}\sigma_{230}-\sigma_{232} \\
\left(\mathrm{~cm}^{-1}\right)\end{array}$} \\
\hline & & \multirow{2}{*}{$\begin{array}{c}\sigma_{\mathrm{vac}} \\
\left(\mathrm{cm}^{-1}\right)\end{array}$} & \multirow{2}{*}{$\begin{array}{c}\Delta \sigma_{\mathrm{Vac}} \\
\left(\mathrm{cm}^{-1}\right)\end{array}$} & & & & & & & & \\
\hline & & & & & & Type & $p$ & $n$ & $g_{1}$ & $g_{2}$ & \\
\hline$M$ & 3122.34 & 32018.0 & -0.1 & $98^{\circ} \cdot 124$ & $M$ & 6 & $(0.395)$ & & & & \\
\hline$M$ & 3121.01 & 32031.7 & 0.0 & $55^{\circ}-124$ & M & 5 & & 1.243 & $(0.982)$ & 1.056 & \\
\hline V & 3120.91 & 32032.7 & 0.0 & $94^{\circ} \cdot 116$ & & & & & & & \\
\hline$M$ & 3119.484 & 32047.32 & +0.19 & $145^{\circ} .91$ & $M$ & 4 & & 0.907 & 1.159 & $(1.232)$ & 0.44 \\
\hline$M$ & 3117.661 & 32066.06 & 0.00 & $15^{\circ} \cdot 30$ & M & 6 & $(0.659)$ & 0.704 & 0.489 & 0.928 & 0.31 \\
\hline$M$ & 3116.476 & 32078.25 & +0.01 & $54^{\circ}-121$ & & & & & & & 0.46 \\
\hline K & 3116.299 & 32080.07 & +0.13 & $126^{\circ}-94$ & $\mathrm{~K}$ & & $(0.29)$ & 1.17 & & & \\
\hline C & 3115.2 & 32091 & 0 & $53^{\circ}-36 ?$ & & & & & & & \\
\hline$M$ & 3114.208 & 32101.61 & +0.06 & $122^{\circ} .51$ & $M$ & 5 & & 1.255 & 1.062 & $(0.986)$ & \\
\hline$M$ & 3114.068 & 32103.05 & -0.02 & $53^{\circ}-118$ & $M$ & 5 & $(0.176)$ & 1.786 & 0.599 & 0.939 & \\
\hline$M$ & 3112.087 & 32123.46 & +0.03 & $93^{\circ}-11$ & & & & & & & \\
\hline$M$ & 3111.831 & 32126.13 & +0.04 & $95^{\circ} .79$ & $M$ & & & & $(0.911)$ & 1.115 & \\
\hline$M$ & 3110.022 & 32144.82 & +0.02 & $15^{\circ}-31$ & $M$ & 6 & $(0.782)$ & 0.757 & 0.492 & 1.013 & 0.39 \\
\hline \multirow[t]{2}{*}{$k$} & 3108.304 & 32162.59 & -0.09 & $130^{\circ}-173$ & $K, L$ & & $(0)$ & 1.30 & & & 0.38 \\
\hline & & & +0.03 & $59^{\circ}-131$ & & & & & & & \\
\hline$K$ & 3107.35 & 32172.5 & 0.0 & $15^{\circ} .73$ & & & & & & & \\
\hline$M$ & 3107.029 & 32175.78 & +0.01 & $15^{\circ} \cdot 8$ & $M$ & 4 & $(0.229)$ & 0.260 & 0.487 & 0.943 & 0.43 \\
\hline$M$ & 3106.691 & 32179.26 & -0.05 & $132^{\circ}-121$ & & & & & & & \\
\hline $\mathrm{K}$ & 3105.752 & 32189.01 & -0.03 & $96^{\circ} \cdot 156$ & $K, L$ & & $(0)$ & 1.01 & & & 0.34 \\
\hline$M$ & 3105.477 & 32191.86 & 0.00 & $42^{\circ}-1$ & $M$ & & & 1.077 & 1.422 & $(2.112)$ & \\
\hline$M$ & 3105.053 & 32196.23 & -0.06 & $95^{\circ}-155$ & & & & & & & \\
\hline$M$ & 3102.666 & 32221.03 & -0.02 & $93^{\circ} .112$ & $M$ & 6 & $(1.296)$ & 0.904 & 0.721 & 1.091 & \\
\hline \multirow[t]{2}{*}{$M$} & 3101.691 & 32231.15 & -0.07 & $16^{\circ}-38$ & $M$ & 6 & $(0.577)$ & & $(0.832)$ & 1.219 & \\
\hline & & & & & $K$ & & $(0.58)$ & 1.00 & & & \\
\hline $\mathrm{K}$ & 3100.799 & 32240.43 & -0.01 & $149^{\circ} \cdot 135$ & & & & & & & \\
\hline$M$ & 3099.863 & 32250.16 & 0.00 & $131^{\circ} .117$ & $M$ & 5 & & 1.400 & $(1.086)$ & 1.00 & \\
\hline$k$ & 3099.739 & 32251.45 & +0.17 & $88^{\circ}-94 ?$ & $\mathrm{~K}$ & & $(0)$ & 1.33 & & & \\
\hline$K$ & 3098.386 & 32265.53 & +0.04 & $94^{\circ}-77$ & & & & & & & \\
\hline \multirow[t]{2}{*}{$M$} & 3096.434 & 32285.87 & -0.04 & $95^{\circ}-117$ & $M$ & 6 & $(0.331)$ & & $(0.911)$ & 1.006 & \\
\hline & & & & & $K$ & & $(0.33)$ & 0.98 & & & \\
\hline
\end{tabular}




\begin{tabular}{|c|c|c|c|c|c|c|c|c|c|c|c|}
\hline \multirow{3}{*}{ Reference } & \multirow{3}{*}{$\begin{array}{c}\text { Wave } \\
\text { Length } \\
\lambda(\AA)\end{array}$} & \multicolumn{2}{|c|}{ Frequency } & \multirow{3}{*}{ Transition } & \multirow{3}{*}{ Reference } & \multirow{2}{*}{\multicolumn{5}{|c|}{ Observed Zeeman Pattern }} & \multirow{3}{*}{$\begin{array}{c}\sigma_{230}-\sigma_{232} \\
\left(\mathrm{~cm}^{-1}\right)\end{array}$} \\
\hline & & \multirow{2}{*}{$\begin{array}{c}\sigma_{\mathrm{vac}} \\
\left(\mathrm{cm}^{-1}\right)\end{array}$} & \multirow{2}{*}{$\begin{array}{c}\Delta \sigma_{\mathrm{vac}} \\
\left(\mathrm{cm}^{-1}\right)\end{array}$} & & & & & & & & \\
\hline & & & & & & $T_{y p e}$ & $p$ & $n$ & $g_{1}$ & $g_{2}$ & \\
\hline C & 3096.29 & 32287.4 & +0.1 & $17^{\circ} .85$ & & & & & & & \\
\hline$M$ & 3095.764 & 32292.86 & -0.04 & $20^{\circ}-46$ & $M$ & 6 & $(0.406)$ & & $(1.277)$ & 1.005 & \\
\hline K & 3093.26 & 32319.0 & 0.0 & $51^{\circ}-107$ & & & & & & & \\
\hline K & 3092.96 & 32322.1 & -0.1 & $98^{\circ}-125$ & & & & & & & \\
\hline$M$ & 3092.35 & 32328.5 & -0.1 & $162^{\circ}-181$ & & & & & & & \\
\hline 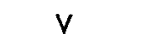 & 3091.66 & 32335.7 & 0.0 & $55^{\circ}-125$ & & & & & & & \\
\hline$M$ & 3090.102 & 3235203 & -0.06 & $78^{\circ}-14$ & $M$ & 5 & $(0.279)$ & 1.984 & 1.145 & 0.581 & \\
\hline$M$ & 3089.631 & 32356.93 & -0.02 & $56^{\circ} \cdot 89$ & & & & & & & \\
\hline$M$ & 3088.842 & 32365.22 & +0.03 & $13^{\circ}-17$ & & & & & & & \\
\hline$M$ & 3088.473 & 32369.09 & +0.01 & $52^{\circ}-75$ & $M$ & 6 & $(0.036)$ & 1.071 & 1.064 & 1.079 & 0.40 \\
\hline$K$ & 3087.09 & 32383.6 & -0.2 & $132^{\circ}-158^{?}$ & & & & & & & \\
\hline$c$ & 3086.57 & 32389.1 & -0.1 & $16^{\circ}-11$ & & & & & & & \\
\hline$M$ & 3084.28 & 32413.1 & 0.0 & $98^{\circ}-126$ & & & & & & & \\
\hline$M$ & 3083.852 & 32417.56 & -0.13 & $55^{\circ}-40$ & $M$ & 4 & $(0.184)$ & $0.463^{?}$ & $(0.982)$ & 1.346 & \\
\hline$M$ & 3083.300 & 3242340 & 000 & $54^{\circ}-81$ & $M$ & 6 & $(0.270)$ & & 1.087 & 0.979 & 0.5 \\
\hline \multirow[t]{2}{*}{$M$} & 3083.004 & 32426.48 & -0.09 & $55^{\circ}-126$ & $M$ & 5 & $(0.099)$ & & $(0982)$ & 1.184 & \\
\hline & & & 0.00 & $152^{\circ} \cdot 190$ & & & & & & & \\
\hline \multirow[t]{2}{*}{$M$} & 3081.982 & 32437.26 & 0.00 & $116^{\circ} .91$ & $M$ & 6 & $(0.318)$ & & 1.141 & $(1.232)$ & \\
\hline & & & & & $\mathrm{K}$ & & $(0.28)$ & 1.20 & & & \\
\hline$M$ & 3081.662 & 32440.63 & +0.06 & $127^{\circ}-135$ & $M$ & $7 b$ & & 1.212 & 1.254 & $(1.242)$ & \\
\hline$V$ & 3081.03 & 32447.3 & 0.0 & $47^{\circ}-54$ & & & & & & & \\
\hline$K$ & 3080.220 & 32455.82 & -0.02 & $151^{\circ} \cdot 187$ & $K, L$ & & $(0)$ & 1.11 & & & 0.42 \\
\hline K & 3079.888 & 32459.31 & +0.04 & $15^{\circ} \cdot 32$ & & & & & & & \\
\hline$M$ & 3078.832 & 32470.45 & -0.02 & $116^{\circ} \cdot 50$ & $M$ & $7 b$ & & 1.104 & 1.146 & $(1.163)$ & 0.44 \\
\hline $\mathrm{K}$ & 3078.744 & 32471.37 & +0.08 & $91^{\circ} .95$ & & & & & & & \\
\hline$M$ & 3077.936 & 32479.92 & -0.01 & $96^{\circ}-157$ & $k$ & & $(0)$ & 0.96 & & & \\
\hline$M$ & 3077.343 & 32486.16 & -0.03 & $95^{\circ}-118$ & $M$ & 6 & $(0.083)$ & 0.928 & 0.916 & 0.940 & 0.47 \\
\hline$M$ & 3076.050 & 32499.79 & -0.05 & $60^{\circ}-132$ & $M$ & 4 & $(0.122)$ & 0.610 & 1.464 & 1.220 & \\
\hline
\end{tabular}

* These data are inconsistent with the classifications listed. 
Table 6 (cont inued)

\begin{tabular}{|c|c|c|c|c|c|c|c|c|c|c|c|}
\hline \multirow{3}{*}{ Reference } & \multirow{3}{*}{$\begin{array}{c}\text { Wave } \\
\text { Length } \\
\lambda(\AA)\end{array}$} & \multicolumn{2}{|c|}{ Frequency } & \multirow{3}{*}{ Transition } & \multirow{3}{*}{ Reference } & \multirow{2}{*}{\multicolumn{5}{|c|}{ Observed Zeeman Pattern }} & \multirow{3}{*}{$\begin{array}{c}\sigma_{230}-\sigma_{232} \\
\left(\mathrm{~cm}^{-1}\right)\end{array}$} \\
\hline & & \multirow{2}{*}{$\begin{array}{c}\sigma_{\text {vac }} \\
\left(\mathrm{cm}^{-1}\right)\end{array}$} & \multirow{2}{*}{$\begin{array}{l}\Delta \sigma_{\text {vac }} \\
\left(\mathrm{cm}^{-1}\right)\end{array}$} & & & & & & & & \\
\hline & & & & & & Type & $p$ & $n$ & $g_{1}$ & $g_{2}$ & \\
\hline$K$ & 3075.29 & 32507.8 & 0.0 & $86^{\circ}-92$ & & & & & & & \\
\hline$\checkmark$ & 3073.42 & 32527.6 & +0.2 & $87^{\circ} \cdot 93 ?$ & & & & & & & \\
\hline M & 3072.831 & 32533.86 & -0.06 & $99^{\circ}-128$ & & & & & & & \\
\hline$M$ & 3072.120 & 32541.39 & -0.03 & $80^{\circ} .91$ & $M$ & 5 & $(0.093)$ & 1.699 & 1.059 & 1.243 & 0.41 \\
\hline $\mathrm{K}$ & 3071.78 & 32545.0 & +0.3 & $94^{\circ} \cdot 78 ?$ & & & & & & & \\
\hline $\mathrm{K}$ & 3070.818 & 32555.18 & +0.03 & $132^{\circ}-179$ & $\mathrm{~L}$ & & $(0)$ & 1.31 & & & \\
\hline$M$ & 3068.987 & 32574.60 & -0.03 & $80^{\circ}-50$ & $M$ & 6 & $(0.276)$ & 1.123 & 1.068 & 1.178 & \\
\hline M & 3066.420 & 32601.87 & -0.04 & $88^{\circ} \cdot 18$ & $M$ & $7 \mathrm{~b}$ & $(0)$ & 1.020 & 0.993 & $(0.977)$ & \\
\hline$M$ & 3065.935 & 32607.00 & -0.04 & $148^{\circ} .92$ & & & & & & & \\
\hline$c$ & 3063.19 & 32636.3 & -0.1 & $85^{\circ} \cdot 16$ & & & & & & & \\
\hline$M$ & 3063.132 & 32636.85 & -0.13 & $53^{\circ} \cdot 119$ & $M$ & 5 & $(0.159)$ & 1.738 & 0.625 & 0.943 & \\
\hline$k$ & 3063.030 & 32637.95 & 0.00 & $133^{\circ}-160$ & $M$ & $6 ?$ & $(0.169)$ & 1.217 & 1.236 & 1.198 & 0.51 \\
\hline$M$ & 3061.703 & 32652.10 & -0.04 & $130^{\circ} \cdot 174$ & $\mathrm{~L}$ & & $(0)$ & 1.25 & & & 0.46 \\
\hline$M$ & 3061.28 & 32656.6 & -0.1 & $58^{\circ}-44$ & & & & & & & \\
\hline $\mathrm{K}$ & 3060.174 & 32668.41 & +0.06 & $94^{\circ}-154$ & $K, L$ & & $(0)$ & 1.06 & & & 0.78 \\
\hline$M$ & 3058.142 & 32690.09 & -0.03 & $78^{\circ}-49$ & & & & & & & \\
\hline$M$ & 3057.904 & 32692.63 & +0.01 & $97^{\circ}-159$ & & & & & & & \\
\hline$M$ & 3057.642 & 32695.44 & -0.05 & $93^{\circ}-73$ & & & & & & & \\
\hline$v$ & 3056.86 & 32703.8 & -0.2 & $98^{\circ}-85 ?$ & & & & & & & \\
\hline$M$ & 3056.099 & 32711.97 & -0.02 & $44^{\circ}-53$ & $M$ & 4 & $(0.218)$ & 0.387 & 1.472 & 1.038 & \\
\hline$M$ & 3054.997 & 32723.77 & -0.01 & $16^{\circ}-39$ & $M$ & 6 & & 1.087 & 0.835 & 1.355 & \\
\hline$M$ & 3051.796 & 32758.10 & -0.07 & $130^{\circ} \cdot 115$ & $M$ & & & 1.091 & $(1.018)$ & 0.997 & \\
\hline$K, M$ & 3050.987 & 32766.78 & +0.01 & $151^{\circ} \cdot 158$ & $\mathrm{~K}$ & & $(0)$ & 1.074 & & & \\
\hline & & & +0.01 & $53^{\circ}-80$ & & & & & & & \\
\hline$M$ & 3049.866 & 32778.80 & -0.11 & $56^{\circ}-90$ & $M$ & 6 & & 1.271 & 1.338 & 1.203 & \\
\hline$M$ & 3049.093 & 32787.11 & +0.01 & $51^{\circ}-108$ & $M$ & $7 b$ & & 0.905 & $(0.857)$ & 0.871 & 0.78 \\
\hline $\mathrm{K}$ & 3048.01 & 32798.8 & +0.2 & $96^{\circ}-120 ?$ & & & & & & & \\
\hline$M$ & 3046.954 & 32810.12 & -0.13 & $21^{\circ}-48 ?$ & & & & & & & 0.43 \\
\hline & & & -0.02 & $162^{\circ}-182$ & & & & & & & \\
\hline 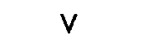 & 3046.16 & 32818.7 & -0.1 & $86^{\circ}-53$ & & & & & & & \\
\hline$M$ & 3045.568 & 32825.08 & 0.00 & $151^{\circ}-188$ & $M$ & 4 & $(0.078)$ & 0.157 & 1.125 & 0.976 & \\
\hline$M$ & 3044.756 & 32833.83 & +0.07 & $2^{\circ}-42$ & $M$ & 5 & $(0.214)$ & 1.169 & 0.530 & 0.957 & \\
\hline$M$ & 3043.249 & 32850.09 & +0.05 & $75^{\circ}-13$ & $M$ & 5 & & & 1.08 & 0.62 & \\
\hline
\end{tabular}


Table 6 (cont inued)

\begin{tabular}{|c|c|c|c|c|c|c|c|c|c|c|c|}
\hline \multirow{3}{*}{ Reference } & \multirow{3}{*}{$\begin{array}{c}\text { Wave } \\
\text { Length } \\
\lambda(\AA)\end{array}$} & \multicolumn{2}{|c|}{ Frequency } & \multirow{3}{*}{ Transition } & \multirow{3}{*}{ Reference } & \multirow{2}{*}{\multicolumn{5}{|c|}{ Observed Zeeman Pattern }} & \multirow{3}{*}{$\begin{array}{c}\sigma_{230}-\sigma_{232} \\
\left(\mathrm{~cm}^{-1}\right)\end{array}$} \\
\hline & & & $\Delta \sigma$ & & & & & & & & \\
\hline & & $\left(\mathrm{cm}^{-1}\right)$ & $\left(\mathrm{cm}^{-1}\right)$ & & & Type & $p$ & $n$ & $g_{1}$ & $g_{2}$ & \\
\hline$M$ & 3043.068 & 32852.04 & -0.06 & $130^{\circ}-151$ & $M$ & 6 & $(0.314)$ & 1.066 & 1.020 & 1.090 & \\
\hline$M$ & 3042.312 & 32860.21 & 0.00 & $84^{\circ}-15$ & $M$ & & $(0.185)$ & & 1.172 & $(0.800)$ & \\
\hline$M$ & 3040.049 & 32884.64 & -0.06 & $52^{\circ}-76$ & $M$ & 6 & $(0.077)$ & 1.036 & 1.051 & 1.021 & \\
\hline $\mathrm{V}$ & 3039.43 & 32891.4 & -0.1 & $52^{\circ}-33$ & & & & & & & \\
\hline$M$ & 3038.600 & 32900.35 & -0.02 & $93^{\circ} \cdot 114$ & $M$ & 6 & $(1.387)$ & 0.909 & 0.715 & 1.111 & \\
\hline$M$ & 3038.005 & 32906.76 & 0.00 & $133^{\circ}-127$ & $M$ & 5 & & 1.357 & $(1.245)$ & 1.213 & \\
\hline \multirow[t]{2}{*}{$K, V$} & 3036.93 & 32918.4 & -0.1 & $124^{\circ}-93$ & & & & & & & \\
\hline & & & -0.1 & $149^{\circ} .94$ & & & & & & & \\
\hline \multirow[t]{2}{*}{$M$} & 3035.542 & 32933.49 & +0.04 & $76^{\circ}-13$ & $M$ & 5 & $(0.243)$ & & 1.10 & 0.61 & \\
\hline & & & -0.01 & $53^{\circ}-120$ & $M$ & 5 & $(0.243)$ & & 0.594 & $(1.080)$ & \\
\hline \multirow[t]{2}{*}{$M$} & 3035.113 & 32938.12 & -0.02 & $151^{\circ}-179$ & $M$ & 6 & $(0.567)$ & & $(1.128)$ & 1.034 & 0.42 \\
\hline & & & & & $K$ & & $(0.52)$ & 1.12 & & & \\
\hline$M$ & & & & & $\mathrm{~L}$ & & $(0)$ & 1.18 & & & \\
\hline$K$ & 3032.88 & 32962.4 & +0.2 & $131^{\circ}-157 ?$ & & & & & & & \\
\hline$M$ & 3031.968 & 32972.28 & -0.10 & $162^{\circ}-189$ & $\mathrm{~K}$ & & $(0.55)$ & 1.22 & & & \\
\hline$M$ & 3031.701 & 32975.19 & 0.00 & $51^{\circ}-69$ & & & & & & & \\
\hline$M$ & 3030.868 & 32984.25 & -0.10 & $131^{\circ} \cdot 119$ & $M$ & 5 & & 1.637 & $(1.086)$ & 0.929 & \\
\hline$K$ & 3029.62 & 32997.9 & -0.1 & $95^{\circ}-157$ & & & & & & & \\
\hline$M$ & 3028.580 & 33009.19 & +0.04 & $15^{\circ}-75$ & $M$ & 5 & $(0.298)$ & 1.982 & 0.499 & 1.092 & \\
\hline$v$ & 3027.61 & 33019.8 & 0.0 & $94^{\circ}-155$ & & & & & & & \\
\hline$M$ & 3026.580 & 33030.99 & -0.11 & $96^{\circ}-121 ?$ & L & & $(0.27)$ & 1.15 & & & 0.40 \\
\hline$v$ & 3025.14 & 33046.7 & -0.2 & $2^{\circ}-43 ?$ & & & & & & & \\
\hline \multirow[t]{2}{*}{$M$} & 3024.669 & 33051.87 & +0.02 & $44^{\circ}-52$ & $M$ & $4 ?$ & & 1.382 & 1.444 & $(1.419)$ & \\
\hline & & & -0.07 & $51^{\circ}-27 ?$ & & & & & & & \\
\hline$M$ & 3022.097 & 33080.00 & -0.01 & $52^{\circ}-116$ & $M$ & 4 or 5 & & 1.177 & $(1.061)$ & 1.095 & \\
\hline$M$ & 3019.418 & 33109.35 & 0.00 & $94^{\circ} \cdot 117$ & $M$ & 6 & $(0.417)$ & 1.082 & 1.146 & 1.027 & 0.74 \\
\hline$M$ & 3018.51 & 33119.3 & 0.0 & $150^{\circ} \cdot 135$ & $M$ & 6 & $(0.691)$ & 1.164 & 1.101 & 1.257 & \\
\hline$M$ & 3017.47 & 33130.7 & -0.1 & $54^{\circ}-123$ & & & & & & & \\
\hline$M$ & 3014.925 & 33158.69 & 0.00 & $86^{\circ}-52$ & $M$ & 6 & $(0.626)$ & 1.296 & 1.175 & 1.429 & \\
\hline$K$ & 3013.605 & 33173.21 & -0.06 & $48^{\circ}-54$ & $K$ & & $(0)$ & 1.37 & & & \\
\hline
\end{tabular}


Table 6 (cont inued)

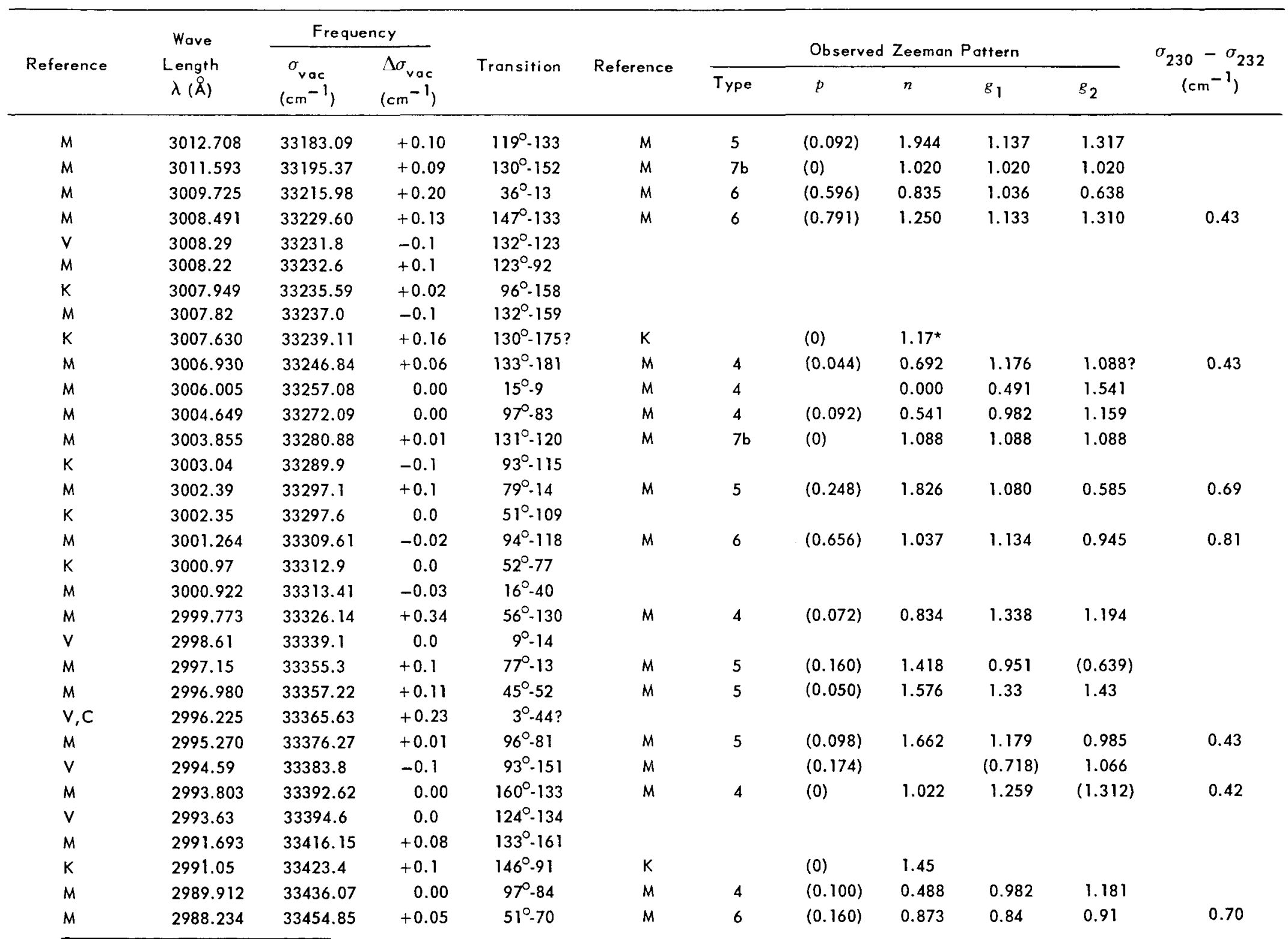


Table 6 (continued)

\begin{tabular}{|c|c|c|c|c|c|c|c|c|c|c|c|}
\hline \multirow{3}{*}{ Reference } & \multirow{3}{*}{$\begin{array}{c}\text { Wave } \\
\text { Length } \\
\lambda(\AA)\end{array}$} & \multicolumn{2}{|c|}{ Frequency } & \multirow{3}{*}{ Transition } & \multirow{3}{*}{ Reference } & \multirow{2}{*}{\multicolumn{5}{|c|}{ Observed Zeeman Pattern }} & \multirow{3}{*}{$\begin{array}{c}\sigma_{230}-\sigma_{232} \\
\left(\mathrm{~cm}^{-1}\right)\end{array}$} \\
\hline & & \multirow{2}{*}{$\begin{array}{c}\sigma_{v o c} \\
\left(\mathrm{~cm}^{-1}\right)\end{array}$} & \multirow{2}{*}{$\begin{array}{l}\Delta \sigma_{\text {vac }} \\
\left(\mathrm{cm}^{-1}\right)\end{array}$} & & & & & & & & \\
\hline & & & & & & Type & $p$ & $n$ & $g_{1}$ & $g_{2}$ & \\
\hline$M$ & 2986.788 & 33471.05 & +0.05 & $43^{\circ}-15$ & $M$ & 6 & $(0.675)$ & 1.026 & 1.254 & 0.804 & \\
\hline$M$ & 2985.246 & 33488.34 & -0.01 & $41^{\circ}-50$ & $M$ & 4 & $(0.060)$ & 1.035 & 1.30 & 1.18 & \\
\hline$v$ & 2984.81 & 33493.2 & -0.1 & $3^{\circ}-45$ & & & & & & & \\
\hline$M$ & 2983.818 & 33504.34 & 0.00 & $162^{\circ} \cdot 190$ & $\mathbf{L}$ & & $(0.19)$ & 1.25 & & & 0.47 \\
\hline$M$ & 2983.566 & 33507.17 & +0.09 & $44^{\circ}-51$ & $M$ & 4 & $(0.240)$ & 0.250 & 1.446 & 0.967 & \\
\hline V & 2983.22 & 33511.1 & -0.1 & $53^{\circ}-81 ?$ & & & & & & & \\
\hline$M$ & 2983.020 & 33513.29 & -0.07 & $131^{\circ}-121$ & & & & & & & \\
\hline$M$ & 2982.018 & 33524.58 & -0.19 & $15^{\circ}-76 ?$ & & & & & & & \\
\hline$M$ & 2981.850 & 33526.44 & -0.14 & $96^{\circ}-122 ?$ & & & & & & & \\
\hline $\mathrm{K}$ & 2981.51 & 33530.3 & -0.2 & $94^{\circ}-156 ?$ & $\mathrm{~K}$ & & $(0)$ & 1.082 & & & \\
\hline$M$ & 2981.362 & 33531.96 & -0.25 & $93^{\circ}-75$ & $M$ & 4 & $(0.200)$ & & $(0.718)$ & 1.094 & \\
\hline$M$ & 2980.338 & 33543.48 & -0.04 & $123^{\circ} .53$ & $M$ & 5 & $(0.106)$ & 1.784 & 1.255 & 1.045 & \\
\hline$M$ & 2976.020 & 33592.15 & +0.07 & $52^{\circ}-78$ & $M$ & 6 & $(0.185)$ & 1.016 & 1.053 & 0.979 & \\
\hline$M$ & 2974.013 & 33614.82 & 0.00 & $51^{\circ} \cdot 71$ & $M$ & 6 & $(0.785)$ & 1.012 & 0.853 & 1.168 & 0.65 \\
\hline$k$ & 2973.544 & 33620.12 & -0.01 & $151^{\circ}-159$ & $K, L$ & & $(0)$ & 1.19 & & & 0.40 \\
\hline$M$ & 2972.932 & 33627.04 & -0.01 & $54^{\circ}-39$ & $M$ & & $(0.137)$ & & (1.088) & 1.349 & \\
\hline$M$ & 2972.224 & 33635.05 & -0.01 & $79^{\circ}-49$ & $M$ & $7 b$ & $(0)$ & 1.068 & 1.068 & 1.068 & \\
\hline \multirow[t]{2}{*}{ M } & 2971.439 & 33643.93 & 0.00 & $117^{\circ}-50$ & $M$ & $4 ?$ & & 1.040 & 1.127 & $(1.163)$ & \\
\hline & & & +0.09 & $57^{\circ}-45$ & & & & & & & \\
\hline V & 2970.61 & 33653.3 & +0.2 & $85^{\circ} \cdot 15 ?$ & & & & & & & \\
\hline $\mathrm{C}$ & 2969.9 & 33661 & 0 & $53^{\circ}-122 ?$ & & & & & & & \\
\hline \multirow[t]{2}{*}{$M$} & 2969.363 & 33667.43 & +0.17 & $130^{\circ}-153 ?$ & $M$ & 6 & $(0.712)$ & & $(1.018)$ & 1.176 & \\
\hline & & & & & $\mathrm{K}$ & & $(0.63)$ & 1.05 & & & \\
\hline$M$ & 2969.069 & 33670.76 & -0.05 & $87^{\circ} \cdot 92$ & & & & & & & \\
\hline$M$ & 2968.685 & 33675.14 & +0.03 & $51^{\circ}-110$ & $M$ & 5 & $(0.065)$ & 1.295 & 0.868 & 0.990 & 0.63 \\
\hline $\mathrm{K}$ & 2966.53 & 33699.6 & 0.0 & $81^{\circ}-91$ & & & & & & & \\
\hline$v$ & 2966.16 & 33703.8 & +0.2 & $19^{\circ}-46 ?$ & & & & & & & \\
\hline$M$ & 2965.501 & 33711.30 & -0.03 & $130^{\circ}-116$ & $M$ & 4 & & 0.748 & $(1.018)$ & 1.095 & \\
\hline$M$ & 2964.008 & 33728.25 & -0.06 & $133^{\circ} \cdot 182$ & & & & & & & \\
\hline$M$ & 2963.609 & 33732.82 & +0.03 & $81^{\circ}-50$ & $M$ & $7 b$ & $(0)$ & 1.165 & 1.165 & 1.165 & \\
\hline
\end{tabular}


Table 6 (continued)

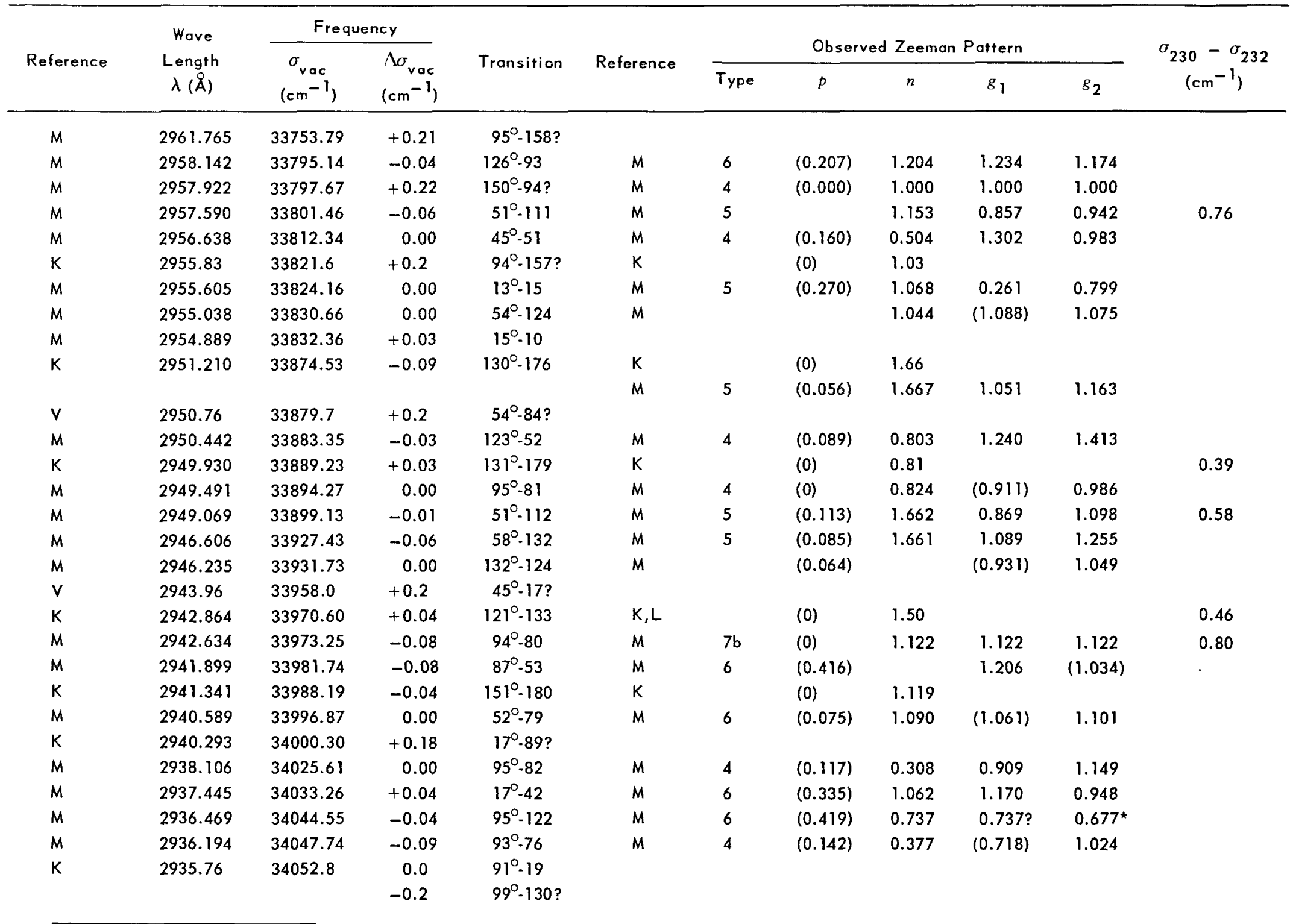


Table 6 (cont inued)

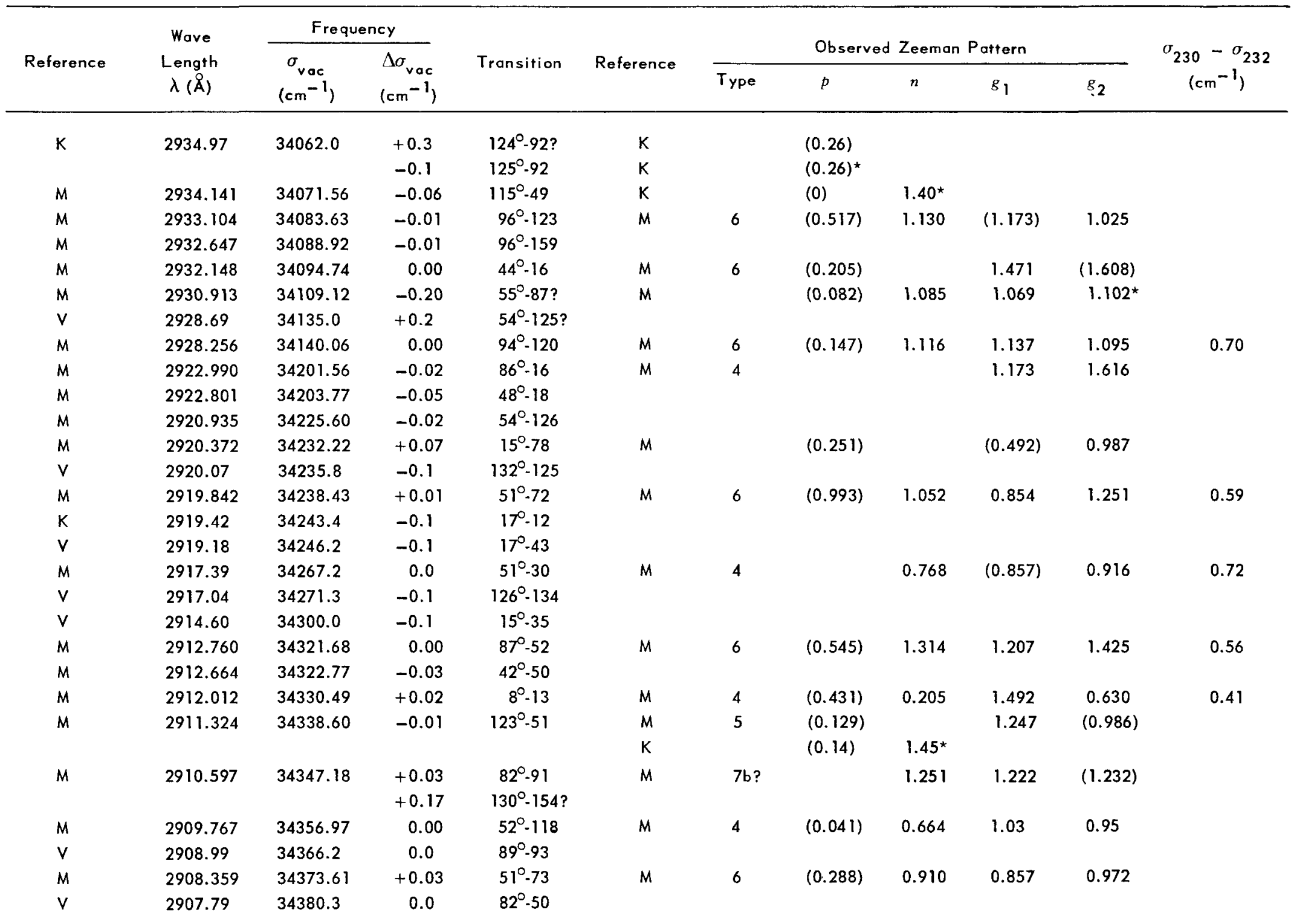

* The se data are inconsistent with the classifications listed. 
Table 6 (cont inved)

\begin{tabular}{|c|c|c|c|c|c|c|c|c|c|c|c|}
\hline \multirow{3}{*}{ Reference } & \multirow{3}{*}{$\begin{array}{c}\text { Wave } \\
\text { Length } \\
\lambda(\AA)\end{array}$} & \multicolumn{2}{|c|}{ Frequency } & \multirow{3}{*}{ Transition } & \multirow{3}{*}{ Reference } & \multirow{2}{*}{\multicolumn{5}{|c|}{ Observed Zeeman Pattern }} & \multirow{3}{*}{$\begin{array}{c}\sigma_{230}-\sigma_{232} \\
\left(\mathrm{~cm}^{-1}\right)\end{array}$} \\
\hline & & \multirow{2}{*}{$\begin{array}{c}\sigma_{v a c} \\
\left(\mathrm{~cm}^{-1}\right)\end{array}$} & \multirow{2}{*}{$\begin{array}{c}\Delta \sigma_{\mathrm{vac}} \\
\left(\mathrm{cm}^{-1}\right)\end{array}$} & & & & & & & & \\
\hline & & & & & & $T_{y p e}$ & $p$ & $n$ & $g_{1}$ & $g_{2}$ & \\
\hline$M$ & 2906.126 & 34400.02 & +0.02 & $45^{\circ}-16$ & & & & & & & \\
\hline$M$ & 2905.934 & 34402.28 & -0.12 & $134^{\circ}-130 ?$ & & & & & & & \\
\hline$v$ & 2904.71 & 34416.8 & -0.1 & $98^{\circ}-89$ & & & & & & & \\
\hline$M$ & 2904.254 & 34422.19 & +0.11 & $17^{\circ}-90$ & $M$ & $7 b$ & & 1.214 & $(1.167)$ & 1.186 & \\
\hline$M$ & 2903.565 & 34430.36 & 0.00 & $55^{\circ}-89$ & $M$ & 6 & $(0.384)$ & 1.054 & 0.977 & 1.131 & \\
\hline$M$ & 2903.170 & 34435.04 & -0.01 & $83^{\circ} .91$ & & & & & & & \\
\hline$M$ & 2899.724 & 34475.96 & 0.00 & $93^{\circ}-77$ & $M$ & 4 & $(0.070)$ & 0.369 & 0.714 & 0.852 & 0.40 \\
\hline$M$ & 2899.373 & 34480.11 & -0.17 & $99^{\circ}-131 ?$ & & & & & & & \\
\hline$M$ & 2897.071 & 34507.52 & +0.04 & $101^{\circ}-132$ & $M$ & 6 & $(0.164)$ & 1.288 & 1.312 & 1.264 & \\
\hline$K$ & 2895.76 & 34523.2 & -0.3 & $130^{\circ}-177 ?$ & & & & & & & \\
\hline$M$ & 2895.143 & 34530.51 & -0.04 & $38^{\circ}-14$ & $M$ & 6 & $(0.369)$ & 0.703 & 0.822 & 0.582 & 0.55 \\
\hline$M$ & 2892.175 & 34565.95 & +0.05 & $131^{\circ}-123$ & $M$ & 5 & & 1.304 & 1.092 & 1.031 & \\
\hline$M$ & 2890.893 & 34581.27 & -0.01 & $13^{\circ}-1$ & & & & & & & \\
\hline$v$ & 2889.19 & 34601.7 & 0.0 & $95^{\circ}-123$ & & & & & & & \\
\hline \multirow[t]{2}{*}{$M$} & 2887.821 & 34618.04 & 0.00 & $118^{\circ}-91$ & $M$ & 6 & $(0.464)$ & & 1.099 & $(1.232)$ & 0.43 \\
\hline & & & & & $K$ & & $(0.48)$ & $1.38^{\star}$ & & & \\
\hline$K$ & 2886.51 & 34633.8 & 0.0 & $149^{\circ} .93$ & $\mathrm{~K}$ & & $(0)$ & 0.95 & & & \\
\hline$M$ & 2886.248 & 34636.93 & -0.01 & $15^{\circ}-79$ & $M$ & 5 & $(0.304)$ & 2.005 & 0.501 & 1.104 & \\
\hline$K$ & 2885.04 & 34651.4 & +0.2 & $118^{\circ}-50 ?$ & $K, L$ & & $(0)$ & 1.02 & & & 0.57 \\
\hline \multirow[t]{2}{*}{$M$} & 2884.295 & 34660.38 & -0.04 & $51^{\circ}-32$ & $M$ & 5 & $(0.040)$ & & $(0.857)$ & 0.738 & 0.68 \\
\hline & & & & & $\mathrm{K}$ & & $(0.06)$ & 1.08 & & & \\
\hline$M$ & 2883.628 & 34668.40 & 0.00 & $96^{\circ}-83$ & $M$ & 4 or 5 & & 1.190 & $(1.173)$ & 1.16 & \\
\hline$M$ & 2882.516 & 34681.77 & -0.01 & $46^{\circ}-51$ & $M$ & 4 & $(0.291)$ & 0.109 & 1.567 & 0.985 & \\
\hline$M$ & 2882.014 & 34687.81 & +0.06 & $16^{\circ}-41$ & $M$ & 6 & $(0.083)$ & 0.856 & 0.828 & 0.883 & 0.47 \\
\hline$M$ & 2881.147 & 34698.22 & -0.17 & $130^{\circ}-155 ?$ & $M$ & $6 ?$ & $(0.092)$ & 1.005 & 1.026 & 0.984 & \\
\hline \multirow[t]{2}{*}{$M$} & 2879.533 & 34717.68 & -0.02 & $94^{\circ}-81$ & $M$ & 5 & $(0.070)$ & 1.509 & 1.136 & 0.987 & \\
\hline & & & +0.04 & $136^{\circ}-132$ & & & & & & & \\
\hline$M$ & 2879.205 & 34721.65 & +0.02 & $40^{\circ}-14$ & $M$ & 6 & $(0.789)$ & 0.848 & 1.107 & 0.586 & \\
\hline
\end{tabular}




\begin{tabular}{|c|c|c|c|c|c|c|c|c|c|c|c|}
\hline \multirow{3}{*}{ Reference } & \multirow{3}{*}{$\begin{array}{c}\text { Wove } \\
\text { Length } \\
\lambda(\AA)\end{array}$} & \multicolumn{2}{|c|}{ Frequency } & \multirow{3}{*}{ Transition } & \multirow{3}{*}{ Reference } & \multirow{2}{*}{\multicolumn{5}{|c|}{ Observed Zeeman Pattern }} & \multirow{3}{*}{$\begin{array}{c}\sigma_{230}-\sigma_{232} \\
\left(\mathrm{~cm}^{-1}\right)\end{array}$} \\
\hline & & \multirow{2}{*}{$\begin{array}{c}\sigma_{\mathrm{vac}} \\
\left(\mathrm{cm}^{-1}\right)\end{array}$} & \multirow{2}{*}{$\begin{array}{l}\Delta \sigma_{\text {vac }} \\
\left(\mathrm{cm}^{-1}\right)\end{array}$} & & & & & & & & \\
\hline & & & & & & Type & $p$ & $n$ & $g_{1}$ & $g_{2}$ & \\
\hline M & 2876.421 & 34755.25 & +0.04 & $93^{\circ}-78$ & M & 4 & $(0.128)$ & 0.046 & 0.705 & 0.963 & \\
\hline M & 2874.630 & 34776.91 & 0.00 & $87^{\circ} .51$ & $M$ & 6 & $(0.540)$ & 1.099 & 1.208 & 0.989 & \\
\hline M & 2873.717 & 34787.95 & -0.06 & $130^{\circ}-117$ & M & $7 b$ & & 1.022 & 1.022 & 1.022 & \\
\hline$M$ & 2870.825 & 34822.98 & $\begin{array}{l}+0.15 \\
-0.03\end{array}$ & $\begin{array}{r}97^{\circ}-161 \\
132^{\circ}-127\end{array}$ & & & & & & & \\
\hline M & 2870.413 & 34827.99 & -0.06 & $80^{\circ}-14$ & M & 5 & $(0.228)$ & 1.736 & 1.043 & 0.583 & 0.53 \\
\hline K & 2869.91 & 34834.1 & +0.2 & $127^{\circ}-93 ?$ & k & & $(0.29)$ & 1.10 & & & \\
\hline M & 2868.678 & 34849.06 & +0.01 & $94^{\circ}-82$ & M & $7 \mathrm{~b}$ & & 1.090 & 1.132 & 1.149 & \\
\hline M & 2868.401 & 34852.42 & +0.10 & $55^{\circ}-90$ & M & 6 & $(0.523)$ & 1.088 & 0.982 & 1.191 & \\
\hline$v$ & 2867.08 & 34868.5 & -0.1 & $38^{\circ}-49$ & & & & & & & \\
\hline M & 2866.412 & 34876.61 & 0.00 & $51^{\circ}-74$ & M & 6 & $(0.707)$ & 0.996 & 0.849 & 1.137 & \\
\hline M & 2865.230 & 34890.96 & +0.08 & $52^{\circ}-119$ & M & 4 & $(0.063)$ & 0.617 & 0.932 & 1.058 & \\
\hline$\checkmark$ & 2862.61 & 34922.9 & 0.0 & $39^{\circ}-49$ & & & & & & & \\
\hline M & 2861.416 & 34937.50 & +0.12 & $54^{\circ} \cdot 86$ & M & $7 \mathrm{~b}$ & (0) & 1.078 & 1.078 & 1.078 & \\
\hline M & 2861.338 & 34938.45 & -0.12 & $126^{\circ} .92$ & M & 6 & $(0.986)$ & 1.096 & 1.232 & 0.951 & \\
\hline K & 2858.92 & 34968.0 & -0.1 & $51^{\circ}-115$ & & & & & & & \\
\hline M & 2857.494 & 34985.45 & -0.01 & $15^{\circ}-36$ & M & 6 & $(1.094)$ & 0.857 & 0.487 & 1.218 & \\
\hline$M$ & 2855.898 & 35005.00 & -0.07 & $16^{\circ}-87$ & M & 5 & $(0.100)$ & 1.321 & 0.857 & 1.043 & \\
\hline$M$ & 2854.920 & 35016.97 & -0.17 & $101^{\circ} \cdot 163 ?$ & & & & & & & \\
\hline K & 2854.63 & 35020.6 & -0.1 & $52^{\circ}-80$ & & & & & & & \\
\hline \multirow[t]{2}{*}{ M } & 2854.131 & 35026.65 & +0.09 & $16^{\circ}-88$ & & & & & & & \\
\hline & & & -0.05 & $99^{\circ}-162$ & & & & & & & \\
\hline M & 2851.445 & 35059.66 & 0.00 & $40^{\circ} .49$ & $M$ & $7 \mathrm{~b} ?$ & & 1.030 & 1.107 & $(1.076)$ & \\
\hline M & 2851.261 & 35061.92 & 0.00 & $116^{\circ}-49$ & $M$ & 5 & & 1.254 & 1.127 & $(1.076)$ & \\
\hline K & 2849.15 & 35087.9 & +0.2 & $96^{\circ}-125 ?$ & $K$ & & $(0.28)$ & & & & \\
\hline \multirow[t]{2}{*}{$M, K$} & 2847.349 & 35110.08 & +0.17 & $88^{\circ}-92 ?$ & & & & & & & \\
\hline & & & +0.08 & $149^{\circ}-134$ & K & & $(0.25)$ & 1.12 & & & \\
\hline M & 2845.83 & 35128.8 & +0.1 & $92^{\circ}-95$ & K & & $(0)$ & 0.90 & & & \\
\hline K & 2843.57 & 35156.8 & -0.2 & $79^{\circ}-13 ?$ & & & & & & & \\
\hline M & 2843.300 & 35160.06 & +0.06 & $93^{\circ} .79$ & M & $5 ?$ & $(0.194)$ & & $(0.718)$ & 1.106 & \\
\hline$v$ & 2843.05 & 35163.2 & +0.2 & $132^{\circ}-181 ?$ & & & & & & & \\
\hline M & 2842.815 & 35166.09 & +0.01 & $80^{\circ}-49$ & M & $7 b$ & & 1.054 & 1.05 & 1.05 & 0.45 \\
\hline$v$ & 2842.67 & 35167.9 & +0.1 & $124^{\circ}-51$ & & & & & & & \\
\hline
\end{tabular}


Table 6 (continued)

\begin{tabular}{|c|c|c|c|c|c|c|c|c|c|c|c|}
\hline \multirow{3}{*}{ Reference } & \multirow{3}{*}{$\begin{array}{c}\text { Wave } \\
\text { Length } \\
\lambda(\AA)\end{array}$} & \multicolumn{2}{|c|}{ Frequency } & \multirow{3}{*}{ Transition } & \multirow{3}{*}{ Reference } & \multirow{2}{*}{\multicolumn{5}{|c|}{ Observed Zeeman Pattern }} & \multirow{3}{*}{$\begin{array}{c}\sigma_{230}-\sigma_{232} \\
\left(\mathrm{~cm}^{-1}\right)\end{array}$} \\
\hline & & \multirow{2}{*}{$\begin{array}{c}\sigma_{\text {vac }} \\
\left(\mathrm{cm}^{-1}\right)\end{array}$} & \multirow{2}{*}{$\begin{array}{l}\Delta \sigma_{\mathrm{vac}} \\
\left(\mathrm{cm}^{-1}\right)\end{array}$} & & & & & & & & \\
\hline & & & & & & Type & $p$ & $n$ & $g_{1}$ & $g_{2}$ & \\
\hline$M$ & 2841.814 & 35178.48 & 0.00 & $96^{\circ}-126$ & $M$ & $7 b$ & $(0)$ & 1.190 & 1.190 & 1.190 & \\
\hline$M$ & 2841.168 & 35186.47 & +0.06 & $95^{\circ}-83$ & $M$ & 4 & $(0.122)$ & 0.302 & 0.912 & 1.156 & \\
\hline$M$ & 2840.156 & 35199.01 & -0.02 & $9^{\circ} \cdot 13$ & $M$ & 4 & $(0.161)$ & 0.473 & 0.970 & 0.640 & \\
\hline$M$ & 2839.335 & 35209.16 & +0.01 & $130^{\circ} \cdot 156$ & & & & & & & \\
\hline$M$ & 2839.243 & 35210.33 & +0.03 & $51^{\circ}-75$ & $M$ & 6 & $(0.582)$ & 0.971 & 0.850 & 1.085 & \\
\hline V & 2838.61 & 35218.2 & -0.2 & $86^{\circ}-15 ?$ & & & & & & & \\
\hline$M$ & 2837.63 & 35230.3 & +0.1 & $93^{\circ} \cdot 155$ & & & & & & & \\
\hline$M$ & 2837.299 & 35234.43 & -0.02 & $161^{\circ} \cdot 133$ & $\mathrm{~L}$ & & $(0)$ & 1.05 & & & 0.31 \\
\hline$M$ & 2836.438 & 35245.14 & -0.02 & $15^{\circ}-37$ & & & & & & & \\
\hline$M$ & 2836.049 & 35249.98 & +0.01 & $119^{\circ}-91$ & $M$ & 6 & $(0.346)$ & 1.170 & 1.121 & 1.220 & \\
\hline$M$ & 2834.484 & 35269.44 & 0.00 & $46^{\circ}-16$ & $M$ & 6 & $(0.050)$ & 1.581 & 1.565 & 1.598 & \\
\hline$M$ & 2833.339 & 35283.69 & +0.02 & $14^{\circ}-17$ & $M$ & 4 & $(0.062)$ & 0.343 & 2.238 & 0.975 & \\
\hline$M$ & 2832.319 & 35296.40 & -0.05 & $147^{\circ}-91$ & $M$ & 4 & & 0.832 & 1.142 & $(1.232)$ & \\
\hline$v$ & 2832.00 & 35300.4 & 0.0 & $90^{\circ}-93$ & & & & & & & \\
\hline$\checkmark$ & 2831.21 & 35310.2 & +0.1 & $127^{\circ}-134$ & & & & & & & \\
\hline$M$ & 2830.445 & 35319.76 & -0.06 & $93^{\circ}-117$ & $M$ & 6 & $(1.019)$ & 0.860 & 0.710 & 1.003 & \\
\hline$M$ & 2829.936 & 35326.12 & +0.01 & $16^{\circ} .89$ & $M$ & 5 & $(0.160)$ & 1.606 & 0.817 & 1.133 & \\
\hline V & 2829.44 & 35332.3 & 0.0 & $132^{\circ} \cdot 161$ & & & & & & & \\
\hline$M$ & 2827.992 & 35350.38 & -0.01 & $95^{\circ}-84$ & & & & & & & \\
\hline$M$ & 2826.858 & 35364.58 & +0.01 & $87^{\circ} \cdot 16$ & $M$ & 4 & $(0.203)$ & 0.591 & 1.201 & 1.605 & \\
\hline$v$ & 2825.94 & 35376.1 & 0.0 & $134^{\circ}-162$ & & & & & & & \\
\hline$K$ & 2823.55 & 35406.0 & 0.0 & $96^{\circ}-160$ & $\mathrm{~K}$ & & $(0)$ & 1.29 & & & \\
\hline$M$ & 2823.12 & 35411.4 & 0.0 & $3^{\circ} \cdot 47$ & & & & & & & \\
\hline V & 2822.68 & 35416.9 & +0.1 & $45^{\circ}-15$ & & & & & & & \\
\hline$C$ & 2822.40 & 35420.4 & +0.1 & $1^{\circ} \cdot 12$ & & & & & & & \\
\hline$M$ & 2822.362 & 35420.90 & -0.02 & $88^{\circ}-53$ & & & & & & & \\
\hline$M$ & 2822.035 & 35425.02 & -0.07 & $94^{\circ}-123$ & $M$ & 6 & $(0.357)$ & 1.095 & 1.146 & 1.044 & 0.68 \\
\hline $\mathrm{K}$ & 2821.61 & 35430.4 & 0.0 & $94^{\circ} \cdot 159$ & $K$ & & $(0)$ & 1.021 & & & \\
\hline$M$ & 2820.337 & 35446.33 & -0.05 & $130^{\circ}-178$ & $M$ & 5 & $(0.074)$ & 1.787 & 1.048 & 1.183 & \\
\hline$M$ & 2819.329 & 35459.02 & 0.00 & $46^{\circ}-2$ & $M$ & 5 & $(0.155)$ & 1.694 & 1.546 & 1.246 & \\
\hline$M$ & 2817.139 & 35486.58 & 0.00 & $97^{\circ}-88$ & $M$ & 4 & & 0.676 & 0.995 & 1.127 & \\
\hline$K$ & 2816.06 & 35500.2 & +0.1 & $130^{\circ}-157$ & $\mathrm{~K}$ & & $(0.32)$ & 1.07 & & & \\
\hline v & 2815.06 & 35512.8 & +0.1 & $150^{\circ}-93$ & & & & & & & \\
\hline
\end{tabular}


Table 6 (continued)

\begin{tabular}{|c|c|c|c|c|c|c|c|c|c|c|c|}
\hline \multirow{3}{*}{ Reference } & \multirow{3}{*}{$\begin{array}{c}\text { Wave } \\
\text { Length } \\
\lambda(\AA)\end{array}$} & \multicolumn{2}{|c|}{ Frequency } & \multirow{3}{*}{ Transition } & \multirow{3}{*}{ Reference } & \multirow{2}{*}{\multicolumn{5}{|c|}{ Observed Zeeman Pattern }} & \multirow{3}{*}{$\begin{array}{c}\sigma_{230}-\sigma_{232} \\
\left(\mathrm{~cm}^{-1}\right)\end{array}$} \\
\hline & & \multirow{2}{*}{$\begin{array}{c}\sigma_{\mathrm{vac}} \\
\left(\mathrm{cm}^{-1}\right)\end{array}$} & \multirow{2}{*}{$\begin{array}{c}\Delta \sigma_{\text {vac }} \\
\left(\mathrm{cm}^{-1}\right)\end{array}$} & & & & & & & & \\
\hline & & & & & & Type & $p$ & $n$ & $g_{1}$ & $g_{2}$ & \\
\hline M & 2814.318 & 35522.13 & -0.07 & $130^{\circ}-119$ & & & & & & & \\
\hline K & 2812.41 & 35546.3 & +0.2 & $151^{\circ}-181$ & $\mathrm{~K}$ & & $(0)$ & 1.130 & & & \\
\hline$k$ & 2810.57 & 35569.5 & 0.0 & $16^{\circ}-12$ & & & & & & & \\
\hline \multirow[t]{2}{*}{ M } & 2808.996 & 35589.44 & 0.00 & $126^{\circ}-52$ & $M$ & 4 & $(0.100)$ & 0.798 & 1.242 & $(1.419)$ & \\
\hline & & & -0.13 & $97^{\circ}-129 ?$ & & & & & & & \\
\hline \multirow[t]{2}{*}{ M } & 2808.878 & 35590.92 & +0.07 & $18^{\circ}-47$ & & & & & & & \\
\hline & & & -0.10 & $54^{\circ}-41$ & & & & & & & \\
\hline \multirow[t]{2}{*}{ M } & 2807.825 & 35604.28 & -0.01 & $53^{\circ}-85$ & M & 6 & $(0.158)$ & 0.631 & 0.600 & 0.663 & \\
\hline & & & & & $K, L$ & & $(0)$ & $1.08^{\star}$ & & & \\
\hline \multirow[t]{2}{*}{ M } & 2807.717 & 35605.63 & -0.03 & $95^{\circ}-125$ & M & 6 & $(0.590)$ & 1.033 & 0.949 & 1.118 & \\
\hline & & & & & $M$ & 6 & $(0.590)$ & 1.033 & $(0.911)$ & 1.074 & \\
\hline$M$ & 2803.381 & 35660.70 & -0.04 & $131^{\circ}-126$ & & & & & & & \\
\hline$M$ & 2802.283 & 35674.70 & +0.11 & $96^{\circ}-127$ & $M$ & 6 & $(0.110)$ & 1.197 & 1.181 & 1.213 & \\
\hline \multirow[t]{2}{*}{$v$} & 2801.92 & 35679.3 & +0.2 & $54^{\circ}-128 ?$ & & & & & & & \\
\hline & & & +0.2 & $100^{\circ}-163 ?$ & & & & & & & \\
\hline$M$ & 2800.572 & 35696.49 & 0.00 & $95^{\circ}-126$ & $M$ & 6 & $(0.943)$ & 1.037 & 0.904 & 1.176 & \\
\hline$v$ & 2800.25 & 35700.6 & +0.2 & $3^{\circ}-48 ?$ & & & & & & & \\
\hline$M$ & 2799.120 & 35715.01 & -0.05 & $84^{\circ}-91$ & $M$ & $7 b$ & & 1.288 & 1.211 & $(1.232)$ & \\
\hline \multirow[t]{2}{*}{$M$} & 2798.270 & 35725.87 & 0.00 & $14^{\circ} \cdot 16$ & $M$ & & $(0.305)$ & 1.302 & 2.216 & 1.607 & \\
\hline & & & -0.02 & $51^{\circ}-76$ & & & & & & & \\
\hline$M$ & 2797.740 & 35732.62 & -0.03 & $51^{\circ}-33$ & $M$ & 5 & & 1.034 & $(0.857)$ & 0.739 & \\
\hline$M$ & 2797.026 & 35741.74 & -0.03 & $41^{\circ}-14$ & $M$ & 6 & $(1.019)$ & 0.933 & 1.265 & 0.582 & \\
\hline $\mathrm{K}$ & 2796.51 & 35748.3 & +0.1 & $84^{\circ}-50$ & $K$ & & $(0)$ & 1.15 & & & \\
\hline$K$ & 2795.53 & 35760.9 & +0.1 & $88^{\circ}-52$ & & & & & & & \\
\hline V & 2795.19 & 35765.2 & +0.2 & $52^{\circ}-81 ?$ & & & & & & & \\
\hline$M$ & 2794.260 & 35777.12 & -0.05 & $149^{\circ}-92$ & $M$ & 5 & $(0.069)$ & 1.553 & 1.098 & 0.965 & \\
\hline V & 2794.01 & 35780.3 & +0.1 & $132^{\circ}-128$ & & & & & & & \\
\hline K & 2793.57 & 35786.0 & +0.1 & $97^{\circ}-89$ & & & & & & & \\
\hline
\end{tabular}

* These data are inconsistent with the classifications listed. 
Table 6 (cont inued)

\begin{tabular}{|c|c|c|c|c|c|c|c|c|c|c|c|}
\hline \multirow{3}{*}{ Reference } & \multirow{3}{*}{$\begin{array}{c}\text { Wave } \\
\text { Length } \\
\lambda(\AA)\end{array}$} & \multicolumn{2}{|c|}{ Frequency } & \multirow{3}{*}{ Transition } & \multirow{3}{*}{ Reference } & \multirow{2}{*}{\multicolumn{5}{|c|}{ Observed Zeeman Pattern }} & \multirow{3}{*}{$\begin{array}{c}\sigma_{230}-\sigma_{232} \\
\left(\mathrm{~cm}^{-1}\right)\end{array}$} \\
\hline & & \multirow{2}{*}{$\begin{array}{c}\sigma_{\mathrm{vac}} \\
\left(\mathrm{cm}^{-1}\right)\end{array}$} & \multirow{2}{*}{$\begin{array}{l}\Delta \sigma_{\text {vac }} \\
\left(\mathrm{cm}^{-1}\right)\end{array}$} & & & & & & & & \\
\hline & & & & & & Type & $p$ & $n$ & $g_{1}$ & $s_{2}$ & \\
\hline$M$ & 2791.016 & 35818.71 & -0.01 & $130^{\circ}-120$ & $M$ & 4 & $(0)$ & 0.778 & $(1.018)$ & 1.087 & \\
\hline$M$ & 2790.425 & 35826.29 & -0.18 & $55^{\circ}-131 ?$ & & & & & & & \\
\hline $\mathrm{v}$ & 2788.09 & 35856.3 & -0.1 & $12^{\circ} \cdot 14$ & & & & & & & \\
\hline$M$ & 2787.132 & 35868.62 & -0.01 & $44^{\circ} \cdot 1$ & $M$ & 4 & $(0.328)$ & 1.136 & 1.454 & 2.100 & \\
\hline$M$ & 2786.26 & 35879.8 & -0.1 & $18^{\circ}-48$ & & & & & & & \\
\hline$M$ & 2785.613 & 35888.16 & -0.09 & $131^{\circ} \cdot 160$ & & & & & & & \\
\hline$M$ & 2785.463 & 35890.12 & -0.12 & $96^{\circ}-86 ?$ & & & & & & & \\
\hline$M$ & 2784.978 & 35896.36 & -0.03 & $52^{\circ} \cdot 82$ & $M$ & 6 & $(0.230)$ & 1.097 & 1.051 & 1.143 & \\
\hline C & 2783.53 & 35915.0 & -0.1 & $17^{\circ}-45$ & & & & & & & \\
\hline \multirow[t]{2}{*}{$M$} & 2783.496 & 35915.44 & +0.02 & $14^{\circ}-2$ & $M$ & 6 & $(0.479)$ & 1.730 & 2.209 & 1.251 & \\
\hline & & & +0.07 & $52^{\circ}-122$ & & & & & & & \\
\hline$M$ & 2783.054 & 35921.17 & -0.06 & $51^{\circ} \cdot 116$ & $M$ & 5 & $(0.124)$ & 1.734 & 0.865 & 1.115 & 0.70 \\
\hline$M$ & 2778.710 & 35977.33 & +0.03 & $127^{\circ}-92$ & $M$ & 6 & $(1.085)$ & 1.089 & 1.257 & 0.947 & \\
\hline$M$ & 2778.027 & 35986.16 & -0.05 & $81^{\circ} \cdot 14$ & & & & & & & 1.23 \\
\hline K & 2777.81 & 35989.0 & +0.1 & $150^{\circ} \cdot 134$ & $\mathrm{~K}$ & & $(0.25)$ & 1.15 & & & \\
\hline$K$ & 2776.20 & 36009.9 & 0.0 & $94^{\circ}-83$ & & & & & & & \\
\hline V & 2775.02 & 36025.2 & 0.0 & $53^{\circ}-86$ & & & & & & & \\
\hline$M$ & 2774.843 & 36027.44 & -0.11 & $151^{\circ}-182$ & & & & & & & \\
\hline$M$ & 2774.51 & 36031.8 & -0.1 & $93^{\circ}-157$ & & & & & & & \\
\hline$K$ & 2774.07 & 36037.5 & 0.0 & $121^{\circ}-91$ & K & & $(0.06)$ & 1.25 & & & \\
\hline$M$ & 2773.960 & 36038.93 & -0.01 & $91^{\circ} .54$ & $M$ & 6 & & 1.231 & 1.231 & 1.231 & \\
\hline$K$ & 2773.54 & 36044.4 & -0.3 & $126^{\circ}-51 ?$ & & & & & & & \\
\hline v & 2773.03 & 36051.0 & -0.2 & $130^{\circ}-121 ?$ & & & & & & & \\
\hline $\mathrm{K}$ & 2771.51 & 36070.8 & 0.0 & $121^{\circ}-50$ & $\mathrm{~K}$ & & $(0)$ & 1.35 & & & 0.67 \\
\hline \multirow[t]{2}{*}{$M$} & 2770.822 & 36079.74 & -0.06 & $41^{\circ}-49$ & $M$ & 4 & $(0.101)$ & 0.785 & 1.252 & 1.064 & \\
\hline & & & & & $\mathrm{L}$ & & $(0)$ & $1.02^{\star}$ & & & \\
\hline$M$ & 2768.848 & 36105.46 & +0.02 & $148^{\circ} \cdot 133$ & $M$ & 6 & $(0.661)$ & 1.240 & 1.167 & 1.314 & 0.45 \\
\hline \multirow[t]{2}{*}{$M$} & 2763.613 & 36173.83 & -0.04 & $45^{\circ}-1$ & $M$ & 4 & $(0.397)$ & 0.916 & 1.313 & 2.107 & \\
\hline & & & 0.00 & $94^{\circ}-84$ & & & & & & & \\
\hline
\end{tabular}

* These data are inconsistent with the classifications listed. 
Table 6 (continued)

\begin{tabular}{|c|c|c|c|c|c|c|c|c|c|c|c|}
\hline \multirow[b]{2}{*}{ Reference } & \multirow{2}{*}{$\begin{array}{c}\text { Wave } \\
\text { Length } \\
\lambda(\AA)\end{array}$} & \multicolumn{2}{|c|}{ Frequency } & \multirow[b]{2}{*}{ Transition } & \multirow[b]{2}{*}{ Reference } & \multicolumn{5}{|c|}{ Observed Zeeman Pattern } & \multirow[b]{2}{*}{$\begin{array}{c}\sigma_{230}-\sigma_{232} \\
\left(\mathrm{~cm}^{-1}\right)\end{array}$} \\
\hline & & $\begin{array}{c}\sigma_{\mathrm{vac}} \\
\left(\mathrm{cm}^{-1}\right)\end{array}$ & $\begin{array}{l}\Delta \sigma_{v a c} \\
\left(\mathrm{~cm}^{-1}\right)\end{array}$ & & & Type & Ubserv & Zeemo & Pattern & $s_{2}$ & \\
\hline \multirow[t]{2}{*}{$\mathrm{K}$} & 2762.84 & 36184.0 & +0.2 & $93^{\circ}-80 ?$ & & & & & & & \\
\hline & & & -0.1 & $96^{\circ} \cdot 161$ & & & & & & & \\
\hline $\mathrm{v}$ & 2762.40 & 36189.7 & -0.1 & $151^{\circ}-189$ & & & & & & & \\
\hline$M$ & 2760.396 & 36216.01 & 0.00 & $88^{\circ}-51$ & $M$ & $7 b$ & (0) & 0.988 & 0.988 & 0.988 & \\
\hline$M$ & 2757.365 & 36255.80 & +0.12 & $130^{\circ}-158$ & $M$ & 6 & $(0.555)$ & & (1.018) & 1.141 & \\
\hline$M$ & 2752.172 & 36324.22 & -0.02 & $81^{\circ} \cdot 49$ & $M$ & 6 & $(0.205)$ & 1.113 & 1.154 & 1.072 & \\
\hline M & 2749.712 & 36356.70 & -0.03 & $133^{\circ}-162$ & & & & & & & \\
\hline M & 2749.542 & 36358.96 & -0.10 & $43^{\circ}-50$ & M & 4 & & 1.059 & 1.234 & $(1.163)$ & 0.58 \\
\hline M & 2749.357 & 36361.40 & -0.07 & $88^{\circ}-17$ & & & & & & & \\
\hline \multirow[t]{2}{*}{$M$} & 2747.853 & 36381.31 & -0.03 & $87^{\circ}-15$ & $M$ & 5 & $(0.209)$ & & 1.203 & $(0.800)$ & \\
\hline & & & & & K & & $(0.21)$ & 1.64 & & & \\
\hline M & 2747.161 & 36390.47 & -0.02 & $38^{\circ}-13$ & M & 6 & $(0.285)$ & 0.731 & 0.824 & 0.636 & 0.61 \\
\hline M & 2745.829 & 36408.13 & -0.12 & $95^{\circ}-86$ & M & 4 & $(0.078)$ & & $(0.911)$ & 1.069 & \\
\hline M & 2743.94 & 36433.2 & -0.1 & $51^{\circ}-78$ & & & & & & & \\
\hline$k$ & 2743.145 & 36443.75 & +0.03 & $90^{\circ}-92$ & L & & (0) & 1.09 & & & \\
\hline M & 2743.067 & 36444.78 & +0.03 & $39^{\circ}-13$ & M & 6 & $(0.813)$ & 0.899 & 1.171 & 0.628 & \\
\hline M & 2739.564 & 36491.41 & -0.13 & $134^{\circ}-132 ?$ & M & & $(0.104)^{\star}$ & & & & \\
\hline K & 2739.13 & 36497.2 & +0.1 & $131^{\circ}-181$ & & & & & & & \\
\hline M & 2738.813 & 36501.39 & +0.10 & $51^{\circ} .35$ & M & & $(0.083)$ & & $(0.857)$ & 1.023 & \\
\hline M & 2738.319 & 36507.97 & 0.00 & $85^{\circ}-91$ & M & 5 & $(0.143)$ & 1.940 & 0.947 & 1.238 & \\
\hline M & 2737.416 & 36520.01 & +0.08 & $94^{\circ}-126$ & M & 6 & $(0.158)$ & 1.147 & 1.125 & 1.170 & \\
\hline K & 2736.19 & 36536.4 & -0.1 & $15^{\circ}-82$ & & & & & & & \\
\hline M & 2735.830 & 36541.18 & 0.00 & $85^{\circ}-50$ & M & 6 & $(0.542)$ & & 0.941 & $(1.163)$ & \\
\hline M & 2734.354 & 36560.91 & +0.08 & $122^{\circ}-91$ & $M$ & 6 & $(0.555)$ & 1.152 & 1.081 & 1.240 & 0.51 \\
\hline$v$ & 2733.22 & 36576.1 & -0.1 & $42^{\circ}-14$ & & & & & & & \\
\hline M & 2732.815 & 36581.50 & -0.07 & $40^{\circ}-13$ & M & 6 & $(0.704)$ & 0.867 & 1.102 & 0.638 & 0.62 \\
\hline$v$ & 2732.70 & 36583.0 & 0.0 & $93^{\circ}-121$ & & & & & & & \\
\hline$M$ & 2730.267 & 36615.63 & 0.00 & $89^{\circ}-51$ & M & 6 & $(0.891)$ & 1.166 & 1.355 & 0.999 & \\
\hline$M$ & 2729.330 & 36628.20 & +0.03 & $127^{\circ}-52$ & $M$ & 4 & $(0.083)$ & 0.871 & 1.266 & 1.424 & \\
\hline$M$ & 2728.915 & 36633.77 & -0.01 & $82^{\circ}-14$ & $M$ & 5 & $(0.319)$ & 2.177 & 1.216 & 0.577 & \\
\hline M & 2727.600 & 36651.44 & +0.10 & $54^{\circ}-90$ & M & & & & (1.088) & 1.189 & \\
\hline M & 2727.24 & 36656.3 & +0.2 & $150^{\circ} .92$ & $M$ & 5 & $(0.069)$ & 1.560 & 1.088 & 0.953 & \\
\hline
\end{tabular}

* These data are inconsistent with the classifications listed. 
Table 6 (cont inued)

\begin{tabular}{|c|c|c|c|c|c|c|c|c|c|c|c|}
\hline \multirow{3}{*}{ Reference } & \multirow{3}{*}{$\begin{array}{c}\text { Wave } \\
\text { Length } \\
\lambda(\AA)\end{array}$} & \multicolumn{2}{|c|}{ Frequency } & \multirow{3}{*}{ Transition } & \multirow{3}{*}{ Reference } & \multirow{2}{*}{\multicolumn{5}{|c|}{ Observed Zeeman Pattern }} & \multirow{3}{*}{$\begin{array}{c}\sigma_{230}-\sigma_{232} \\
\left(\mathrm{~cm}^{-1}\right)\end{array}$} \\
\hline & & & $\Delta \sigma$ & & & & & & & & \\
\hline & & $\left(\mathrm{cm}^{-1}\right)$ & $\left(\mathrm{cm}^{-1}\right)$ & & & Type & $p$ & $n$ & $g_{1}$ & $g_{2}$ & \\
\hline$M$ & 2726.490 & 36666.34 & -0.03 & $131^{\circ} .161$ & & & & & & & \\
\hline$M$ & 2725.568 & 36678.76 & -0.01 & $53^{\circ}-41$ & $M$ & 4 & $(0.146)$ & 0.012 & $(0.601)$ & 0.895 & \\
\hline$M$ & 2724.886 & 36687.94 & -0.05 & $80^{\circ}-13$ & $M$ & 5 & $(0.204)$ & 1.656 & 1.050 & 0.642 & \\
\hline \multirow[t]{2}{*}{$M$} & 2722.383 & 36721.67 & -0.02 & $83^{\circ} \cdot 14$ & $M$ & 5 & $(0.260)$ & 1.892 & 1.111 & 0.591 & \\
\hline & & & -0.08 & $151^{\circ}-190$ & & & & & & & \\
\hline$M$ & 2721.697 & 36730.92 & +0.01 & $123^{\circ}-133$ & $M$ & 5 & & 1.483 & 1.263 & $(1.312)$ & \\
\hline$M$ & 2720.829 & 36742.64 & 0.00 & $14^{\circ}-15$ & $M$ & 4 & $(0.709)$ & 0.078 & 2.205 & 0.787 & \\
\hline K & 2720.48 & 36747.4 & -0.1 & $94^{\circ} \cdot 160$ & & & & & & & \\
\hline \multirow[t]{2}{*}{$M$} & 2719.939 & 36754.64 & -0.07 & $90^{\circ}-53$ & $M$ & 6 & $(0.296)$ & 1.089 & 1.149 & 1.031 & \\
\hline & & & -0.15 & $97^{\circ}-130 ?$ & & & & & & & \\
\hline$M$ & 2719.465 & 36761.07 & -0.02 & $89^{\circ} \cdot 17$ & $M$ & 5 & $(0.189)$ & 1.908 & 1.344 & 0.967 & \\
\hline$M$ & 2716.318 & 36803.65 & -0.02 & $88^{\circ}-16$ & $M$ & 4 & $(0.300)$ & 0.070 & 0.993 & 1.602 & \\
\hline v & 2712.07 & 36861.3 & +0.1 & $96^{\circ} \cdot 87$ & & & & & & & \\
\hline V & 2710.48 & 36882.9 & 0.0 & $96^{\circ}-88$ & & & & & & & \\
\hline$M$ & 2708.181 & 36914.23 & -0.02 & $42^{\circ}-49$ & $M$ & 4 & $(0.174)$ & 0.553 & 1.409 & 1.065 & \\
\hline$M$ & 2707.54 & 36923.0 & +0.1 & $2^{\circ}-48$ & $M$ & 5 & $(0.372)$ & 1.633 & 0.510 & 1.257 & \\
\hline$M$ & 2704.503 & 36964.43 & +0.28 & $1^{\circ}-44$ & $M$ & 5 & $(0.347)$ & 1.260 & 0.23 & 0.92 & \\
\hline$M$ & 2703.962 & 36971.82 & +0.01 & $82^{\circ}-49$ & $M$ & 6 & $(0.356)$ & 1.155 & 1.226 & 1.084 & \\
\hline$M$ & 2702.933 & 36985.90 & 0.00 & $96^{\circ} \cdot 129$ & $M$ & 6 & $(0.273)$ & & $(1.173)$ & 1.095 & \\
\hline$M$ & 2700.605 & 37017.77 & -0.01 & $53^{\circ}-88$ & $M$ & 6 & $(1.280)$ & 0.857 & 0.602 & 1.111 & \\
\hline$M$ & 2698.742 & 37043.33 & 0.00 & $46^{\circ}-1$ & $M$ & 4 & $(0.272)$ & 1.291 & 1.562 & 2.105 & \\
\hline \multirow[t]{2}{*}{$M$} & 2697.550 & 37059.70 & -0.01 & $83^{\circ} \cdot 49$ & $M$ & 6 & $(0.060)$ & 1.092 & 1.104 & 1.080 & \\
\hline & & & +0.18 & $93^{\circ}-82 ?$ & & & & & & & \\
\hline$M$ & 2696.836 & 37069.51 & +0.02 & $91^{\circ} \cdot 18$ & $M$ & 5 & $(0.118)$ & 1.539 & 1.190 & 0.957 & \\
\hline$v$ & 2696.18 & 37078.5 & 0.0 & $93^{\circ}-122$ & & & & & & & \\
\hline$M$ & 2695.822 & 37083.45 & +0.05 & $127^{\circ}-51$ & $M$ & & $(0.132)$ & & 1.260 & $(0.986)$ & \\
\hline \multirow[t]{2}{*}{$M$} & 2695.207 & 37091.89 & -0.10 & $47^{\circ}-51$ & $K$ & & $(0.09)$ & 0.73 & & & \\
\hline & & & -0.13 & $1^{\circ}-45 ?$ & & & & & & & \\
\hline$K$ & 2695.02 & 37094.5 & -0.1 & $90^{\circ}-52$ & & & & & & & \\
\hline$M$ & 2694.347 & 37103.75 & 0.00 & $130^{\circ}-123$ & $M$ & $7 b$ & & 1.022 & 1.022 & 1.022 & \\
\hline$M$ & 2693.635 & 37113.55 & +0.28 & $16^{\circ}-44$ & $M$ & 6 & $(0.134)$ & 0.881 & 0.837 & 0.926 & \\
\hline$M$ & 2693.111 & 37120.78 & -0.01 & $53^{\circ} \cdot 129$ & $M$ & & $(0.240)$ & & $(0.601)$ & 1.084 & \\
\hline$M$ & 2692.422 & 37130.28 & -0.07 & $10^{\circ}-13$ & $M$ & 5 & & 0.690 & 0.537 & $(0.639)$ & 0.48 \\
\hline
\end{tabular}


Table 6 (cont inued)

\begin{tabular}{|c|c|c|c|c|c|c|c|c|c|c|c|}
\hline \multirow[b]{2}{*}{ Reference } & \multirow{2}{*}{$\begin{array}{c}\text { Wave } \\
\text { Length } \\
\lambda(\AA)\end{array}$} & \multicolumn{2}{|c|}{ Frequency } & \multirow[b]{2}{*}{ Transition } & \multirow[b]{2}{*}{ Reference } & \multicolumn{5}{|c|}{ Observed Zeeman Pattern } & \multirow[b]{2}{*}{$\begin{array}{c}\sigma_{230}-\sigma_{232} \\
\left(\mathrm{~cm}^{-1}\right)\end{array}$} \\
\hline & & $\begin{array}{c}\sigma_{\mathrm{vac}} \\
\left(\mathrm{cm}^{-1}\right)\end{array}$ & $\begin{array}{l}\Delta \sigma_{\text {voc }} \\
\left(\mathrm{cm}^{-1}\right)\end{array}$ & & & Type & Ubserv & Leeman & attern & $g_{2}$ & \\
\hline M & 2689.377 & 37172.31 & 0.00 & $52^{\circ}-124$ & M & $7 b$ & & 1.096 & $(1.061)$ & 1.072 & \\
\hline \multirow[t]{2}{*}{$K, V$} & 2688.66 & 37182.2 & 0.0 & $96^{\circ}-89$ & & & & & & & \\
\hline & & & +0.2 & $97^{\circ}-131 ?$ & & & & & & & \\
\hline M & 2688.329 & 37186.80 & +0.19 & $51^{\circ}-36$ & $M$ & 4 & $(0.183)$ & 0.311 & 0.855 & 1.221 & 0.57 \\
\hline \multirow[t]{2}{*}{$\vee$} & 2687.51 & 37198.1 & -0.1 & $51^{\circ}-118$ & & & & & & & \\
\hline & & & -0.1 & $54^{\circ}-130$ & & & & & & & \\
\hline M & 2687.138 & 37203.29 & 0.00 & $89^{\circ} \cdot 16$ & $M$ & 4 & $(0.130)$ & 0.953 & 1.336 & 1.596 & \\
\hline $\mathrm{K}$ & 2684.66 & 37237.6 & +0.2 & $47^{\circ}-17 ?$ & & & & & & & \\
\hline M & 2684.294 & 37242.70 & 0.00 & $118^{\circ}-49$ & $\mathrm{~K}$ & & (0) & 1.134 & & & 0.44 \\
\hline M & 2680.19 & 37299.7 & +0.4 & $132^{\circ}-130$ & M & & $(0.132)$ & & 0.930 & $(1.194)$ & \\
\hline M & 2678.938 & 37317.15 & +0.02 & $53^{\circ}-89$ & M & 6 & $(1.351)$ & 0.860 & 0.589 & 1.132 & \\
\hline M & 2675.670 & 37362.73 & +0.04 & $48^{\circ} \cdot 52$ & $M$ & 5 & $(0.160)$ & 1.880 & 1.14 & 1.43 & \\
\hline M & 2665.892 & 37499.76 & 0.00 & $14^{\circ}-1$ & M & $7 b ?$ & (0) & 2.168 & 2.224 & $(2.112)$ & \\
\hline v & 2664.07 & 37525.4 & -0.2 & $94^{\circ}-161 ?$ & & & & & & & \\
\hline M & 2662.349 & 37549.66 & -0.16 & $90^{\circ}-51$ & M & 6 & $(0.413)$ & 1.067 & 1.146 & 0.981 & \\
\hline M & 2661.38 & 37563.3 & 0.0 & $53^{\circ}-43$ & & & & & & & \\
\hline M & 2658.667 & 37601.66 & -0.05 & $41^{\circ}-13$ & M & 6 & $(0.938)$ & 0.955 & 1.263 & 0.635 & \\
\hline $\mathrm{K}$ & 2658.21 & 37608.1 & +0.1 & $11^{\circ} \cdot 13$ & & & & & & & \\
\hline M & 2656.272 & 37635.56 & 0.00 & $93^{\circ}-123$ & M & 6 & $(1.077)$ & & $(0.718)$ & 1.026 & \\
\hline K & 2655.90 & 37640.8 & 0.0 & $93^{\circ}-159$ & & & & & & & \\
\hline \multirow[t]{2}{*}{ K } & 2653.17 & 37679.6 & +0.2 & $129^{\circ}-95$ & M & 6 & $(0.727)$ & 1.011 & 1.115 & 0.919 & \\
\hline & & & -0.1 & $47^{\circ} \cdot 16$ & & & & & & & \\
\hline M & 2652.073 & 37695.14 & -0.14 & $90^{\circ}-17$ & M & 4 & $(0.092)$ & 1.428 & 1.166 & 0.991 & \\
\hline$M$ & 2650.588 & 37716.26 & -0.11 & $12^{\circ}-13$ & M & $7 \mathrm{~b} ?$ & & 0.616 & 0.685 & $(0.639)$ & 0.57 \\
\hline M & 2649.873 & 37726.43 & -0.13 & $132^{\circ} \cdot 131 ?$ & M & 4 & $(0.041)$ & 0.500 & 0.787 & $0.868 ?$ & \\
\hline M & 2649.481 & 37732.03 & -0.07 & $51^{\circ}-119$ & & & & & & & \\
\hline M & 2643.29 & 37820.4 & 0.0 & $88^{\circ} \cdot 15$ & M & 5 & $(0.099)$ & 1.291 & 1.001 & 0.803 & \\
\hline$M$ & 2641.494 & 37846.10 & -0.05 & $81^{\circ}-13$ & M & 5 & $(0.264)$ & 1.955 & 1.160 & 0.635 & \\
\hline M & 2640.393 & 37861.88 & -0.01 & $51^{\circ}-80$ & M & 6 & $(0.671)$ & & $(0.857)$ & 1.127 & \\
\hline M & 2639.895 & 37869.03 & -0.20 & $47^{\circ}-2$ & K & & $(0)$ & 1.13 & & & \\
\hline M & 2639.508 & 37874.57 & -0.05 & $119^{\circ}-49$ & K & & (0) & $1.00^{*}$ & & & \\
\hline
\end{tabular}

* These data are inconsistent with the classifications listed. 
Table 6 (continued)

\begin{tabular}{|c|c|c|c|c|c|c|c|c|c|c|c|}
\hline \multirow{3}{*}{ Reference } & \multirow{3}{*}{$\begin{array}{c}\text { Wave } \\
\text { Length } \\
\lambda(\AA)\end{array}$} & \multicolumn{2}{|c|}{ Frequency } & \multirow{3}{*}{ Transition } & \multirow{3}{*}{ Reference } & \multirow{2}{*}{\multicolumn{5}{|c|}{ Observed Zeeman Pattern }} & \multirow{3}{*}{$\begin{array}{c}\sigma_{230}-\sigma_{232} \\
\left(\mathrm{~cm}^{-1}\right)\end{array}$} \\
\hline & & \multirow{2}{*}{$\begin{array}{c}\sigma_{v a c} \\
\left(\mathrm{~cm}^{-1}\right)\end{array}$} & \multirow{2}{*}{$\begin{array}{l}\Delta \sigma_{\mathrm{Vac}} \\
\left(\mathrm{cm}^{-1}\right)\end{array}$} & & & & & & & & \\
\hline & & & & & & Type & $p$ & $n$ & $g_{1}$ & $g_{2}$ & \\
\hline \multirow[t]{2}{*}{$M$} & 2633.333 & 37963.38 & 0.00 & $48^{\circ}-17$ & $M$ & 6 & $(0.223)$ & & 1.117 & $(0.968)$ & \\
\hline & & & & & $K$ & & $(0.220)$ & 1.05 & & & \\
\hline$M$ & 2630.825 & 37999.57 & 0.00 & $44^{\circ}-50$ & $M$ & 4 & $(0.154)$ & 0.717 & 1.462 & 1.164 & \\
\hline K & 2630.68 & 38001.7 & 0.0 & $84^{\circ}-14$ & & & & & & & \\
\hline$M$ & 2628.816 & 38028.61 & -0.01 & $51^{\circ} \cdot 120$ & $M$ & 5 & $(0.113)$ & 1.666 & 0.868 & 1.097 & \\
\hline \multirow[t]{2}{*}{$M$} & 2626.403 & 38063.55 & -0.04 & $52^{\circ} \cdot 127$ & $M$ & 5 & $(0.087)$ & 1.586 & 1.086 & 1.229 & \\
\hline & & & +0.03 & $120^{\circ}-49$ & & & & & & & \\
\hline$M$ & 2625.736 & 38073.22 & +0.02 & $86^{\circ}-91$ & $M$ & $5 ?$ & & 1.332 & 1.194 & $(1.232)$ & \\
\hline$M$ & 2623.454 & 38106.33 & -0.08 & $86^{\circ} \cdot 50$ & $M$ & $7 \mathrm{~b}$ & $(0)$ & 1.163 & 1.163 & 1.163 & \\
\hline$M$ & 2622.354 & 38122.31 & +0.10 & $95^{\circ}-90$ & $M$ & & $(0.135)$ & & $(0.911)$ & 1.188 & \\
\hline $\mathrm{K}$ & 2618.90 & 38172.6 & +0.2 & $148^{\circ}-91 ?$ & $K$ & & $(0)$ & 1.06 & & & \\
\hline$v$ & 2617.14 & 38198.3 & -0.2 & $15^{\circ}-40 ?$ & & & & & & & \\
\hline$M$ & 2615.353 & 38224.35 & +0.01 & $94^{\circ} \cdot 88$ & $M$ & 6 & $(0.080)$ & 1.110 & 1.122 & 1.099 & \\
\hline$M$ & 2611.686 & 38278.02 & -0.08 & $49^{\circ}-54$ & $M$ & 5 & $(0.175)$ & 1.738 & 0.924 & 1.250 & \\
\hline$v$ & 2611.13 & 38286.2 & +0.2 & $53^{\circ} \cdot 130 ?$ & & & & & & & \\
\hline$M$ & 2609.855 & 38304.88 & +0.05 & $45^{\circ} \cdot 50$ & $M$ & 4 & $(0.063)$ & 0.965 & 1.280 & 1.154 & \\
\hline$M$ & 2608.327 & 38327.31 & -0.04 & $94^{\circ} \cdot 129$ & $M$ & 6 & $(0.148)$ & 1.104 & 1.125 & 1.083 & \\
\hline$V$ & 2607.78 & 38335.4 & -0.1 & $93^{\circ} \cdot 124$ & & & & & & & \\
\hline$M$ & 2607.482 & 38339.73 & +0.01 & $84^{\circ}-49$ & $M$ & 6 & $(0.227)$ & & 1.167 & $(1.076)$ & \\
\hline$M$ & 2603.011 & 38405.58 & 0.00 & $48^{\circ}-16$ & $M$ & 6 & $(0.738)$ & & 1.116 & $(1.608)$ & \\
\hline$K$ & 2601.62 & 38426.1 & 0.0 & $130^{\circ}-160$ & & & & & & & \\
\hline$M$ & 2600.885 & 38436.97 & 0.00 & $126^{\circ} \cdot 133$ & $M$ & 5 & & 1.519 & 1.253 & $(1.312)$ & \\
\hline$M$ & 2597.050 & 38493.73 & +0.01 & $82^{\circ}-13$ & $M$ & 5 & $(0.291)$ & 2.074 & 1.214 & 0.640 & \\
\hline$M$ & 2595.033 & 38523.65 & -0.04 & $94^{\circ}-89$ & $M$ & $7 b$ & & 1.115 & $(1.132)$ & 1.139 & \\
\hline$M$ & 2590.224 & 38595.16 & 0.00 & $48^{\circ}-2$ & $M$ & $7 b ?$ & $(0.067)$ & 1.054 & 1.121 & 1.255 & \\
\hline$M$ & 2589.063 & 38612.47 & -0.01 & $43^{\circ}-14$ & $M$ & 6 & $(0.993)$ & 0.926 & 1.250 & 0.586 & \\
\hline$M$ & 2587.25 & 38639.5 & -0.1 & $93^{\circ}-125$ & $M$ & 6 & $(1.269)$ & 0.898 & 0.717 & 1.080 & \\
\hline$M$ & 2586.153 & 38665.91 & -0.06 & $151^{\circ}-162$ & $M$ & $7 \mathrm{~b} ?$ & $(0)$ & 1.145 & $(1.128)$ & 1.124 & \\
\hline \multirow[t]{2}{*}{$v, K$} & 2583.455 & 38696.27 & -0.15 & $47^{\circ}-15$ & $M$ & 6 & $(0.657)$ & 0.976 & 1.189 & 0.766 & \\
\hline & & & -0.08 & $92^{\circ}-54$ & & & & & & & \\
\hline$M$ & 2580.700 & 38737.59 & -0.02 & $51^{\circ}-82$ & $M$ & 6 & $(0.751)$ & 1.000 & 0.852 & 1.152 & \\
\hline$M$ & 2579.437 & 38756.56 & -0.03 & $51^{\circ}-122$ & $M$ & 5 & & 1.473 & 0.878 & 1.048 & \\
\hline$M$ & 2576.907 & 38794.60 & 0.00 & $85^{\circ} \cdot 14$ & $M$ & & $(0.182)$ & & 0.950 & $(0.586)$ & \\
\hline
\end{tabular}


Table 6 (continued)

\begin{tabular}{|c|c|c|c|c|c|c|c|c|c|c|c|}
\hline \multirow{3}{*}{ Reference } & \multirow{3}{*}{$\begin{array}{c}\text { Wave } \\
\text { Length } \\
\lambda(\AA)\end{array}$} & \multicolumn{2}{|c|}{ Frequency } & \multirow{3}{*}{ Transition } & \multirow{3}{*}{ Reference } & \multirow{2}{*}{\multicolumn{5}{|c|}{ Observed Zeeman Pattern }} & \multirow{3}{*}{$\begin{array}{c}\sigma_{230}-\sigma_{232} \\
\left(\mathrm{~cm}^{-1}\right)\end{array}$} \\
\hline & & \multirow{2}{*}{$\begin{array}{c}\sigma_{v a c} \\
\left(\mathrm{~cm}^{-1}\right)\end{array}$} & \multirow{2}{*}{$\begin{array}{l}\Delta \sigma_{\mathrm{Vac}} \\
\left(\mathrm{cm}^{-1}\right)\end{array}$} & & & & & & & & \\
\hline & & & & & & Type & $p$ & $n$ & $g_{1}$ & $g_{2}$ & \\
\hline$M$ & 2576.690 & 38797.87 & -0.02 & $123^{\circ} .91$ & $M$ & $7 b$ & $(0)$ & 1.238 & 1.238 & 1.238 & \\
\hline$M$ & 2574.484 & 38831.11 & +0.01 & $123^{\circ}-50$ & $M$ & 5 & & 1.409 & 1.233 & $(1.163)$ & 0.65 \\
\hline$M$ & 2566.905 & 38945.75 & +0.10 & $94^{\circ}-90$ & $M$ & 4 & & 1.008 & $(1.132)$ & 1.182 & \\
\hline$M$ & 2566.589 & 38950.55 & +0.04 & $43^{\circ}-49$ & $M$ & 4 & $(0.084)$ & 0.814 & 1.234 & 1.066 & \\
\hline$M$ & 2565.597 & 38965.61 & -0.03 & $13^{\circ}-14$ & $M$ & 5 & $(0.162)$ & 0.736 & 0.262 & 0.586 & \\
\hline$M$ & 2561.937 & 39021.27 & -0.05 & $93^{\circ}-85$ & $M$ & 5 & & 0.788 & $(0.718)$ & 0.690 & \\
\hline V & 2561.04 & 39034.9 & 0.0 & $130^{\circ} \cdot 181$ & & & & & & & \\
\hline$M$ & 2556.480 & 39104.56 & +0.27 & $53^{\circ}-44$ & $M$ & 4 & $(0.157)$ & 0.122 & 0.605 & 0.927 & \\
\hline$V$ & 2554.64 & 39132.7 & +0.1 & $85^{\circ}-49$ & & & & & & & \\
\hline$M$ & 2553.242 & 39154.15 & -0.10 & $90^{\circ}-15$ & $M$ & 5 & $(0.183)$ & 1.686 & 1.158 & 0.804 & \\
\hline$M$ & 2551.931 & 39174.27 & 0.00 & $46^{\circ} \cdot 50$ & $M$ & 4 & $(0.204)$ & 0.551 & 1.552 & 1.152 & \\
\hline$M$ & 2551.200 & 39185.49 & 0.00 & $122^{\circ}-49$ & $M$ & $7 b$ & $(0)$ & 1.073 & 1.073 & 1.073 & \\
\hline \multirow[t]{2}{*}{$M$} & 2547.902 & 39236.21 & +0.02 & $87^{\circ}-91$ & $M$ & $5 ?$ & & 1.280 & 1.21 & $(1.232)$ & \\
\hline & & & & & $L$ & & $(0)$ & 1.22 & & & \\
\hline$M$ & 2545.747 & 39269.42 & +0.02 & $87^{\circ}-50$ & $M$ & 6 & $(0.074)$ & 1.185 & 1.199 & 1.170 & \\
\hline$M$ & 2545.345 & 39275.62 & +0.05 & $149^{\circ}-133$ & $M$ & 6 & $(0.994)$ & 1.205 & 1.091 & 1.312 & \\
\hline$M$ & 2542.642 & 39317.37 & +0.01 & $51^{\circ} \cdot 38$ & $M$ & 4 & $(0.178)$ & 0.322 & 0.851 & 1.205 & \\
\hline$M$ & 2538.940 & 39374.69 & 0.00 & $52^{\circ}-129$ & $M$ & $7 b$ & $(0)$ & 1.107 & $(1.061)$ & 1.075 & \\
\hline $\mathrm{K}$ & 2538.85 & 39376.1 & -0.2 & $92^{\circ}-94 ?$ & & & & & & & \\
\hline$M$ & 2535.870 & 39422.36 & +0.01 & $48^{\circ} \cdot 15$ & $M$ & 6 & $(0.483)$ & & 1.128 & $(0.800)$ & \\
\hline$M$ & 2534.604 & 39442.04 & -0.12 & $93^{\circ}-86$ & $M$ & 4 & $(0.178)$ & 0.180 & 0.719 & 1.080 & \\
\hline 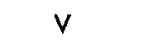 & 2533.87 & 39453.4 & -0.1 & $47^{\circ}-1$ & & & & & & & \\
\hline$M$ & 2532.452 & 39475.56 & -0.14 & $127^{\circ} \cdot 133$ & $M$ & $7 b ?$ & & 1.430 & 1.28 & $(1.312)$ & \\
\hline M & 2526.342 & 39571.03 & 0.00 & $52^{\circ}-89$ & $M$ & 6 & $(0.174)$ & 1.097 & 1.062 & 1.132 & \\
\hline$M$ & 2525.931 & 39577.47 & -0.02 & $91^{\circ}-92$ & $M$ & 4 & $(0.128)$ & 0.323 & 1.203 & 0.951 & \\
\hline$M$ & 2521.78 & 39642.6 & -0.2 & $95^{\circ}-162 ?$ & & & & & & & \\
\hline K & 2520.66 & 39660.2 & -0.1 & $124^{\circ} \cdot 50$ & $\mathrm{~K}$ & & $(0)$ & 0.92 & & & \\
\hline$M$ & 2516.45 & 39726.6 & -0.3 & $92^{\circ} \cdot 18 ?$ & & & & & & & \\
\hline$M$ & 2507.92 & 39861.7 & 0.0 & $84^{\circ}-13$ & $M$ & 5 & $(0.266)$ & 1.973 & 1.172 & 0.639 & \\
\hline$M$ & 2505.615 & 39898.34 & -0.07 & $51^{\circ}-83$ & $M$ & 6 & $(0.757)$ & 1.008 & 0.860 & 1.163 & \\
\hline
\end{tabular}


Table 6 (continued)

\begin{tabular}{|c|c|c|c|c|c|c|c|c|c|c|c|}
\hline \multirow{3}{*}{ Reference } & \multirow{3}{*}{$\begin{array}{c}\text { Wave } \\
\text { Length } \\
\lambda(\AA)\end{array}$} & \multicolumn{2}{|c|}{ Frequency } & \multirow{3}{*}{ Transition } & \multirow{3}{*}{ Reference } & \multirow{2}{*}{\multicolumn{5}{|c|}{ Observed Zeeman Pattern }} & \multirow{3}{*}{$\begin{array}{c}\sigma_{230}-\sigma_{232} \\
\left(\mathrm{~cm}^{-1}\right)\end{array}$} \\
\hline & & \multirow{2}{*}{$\begin{array}{c}\sigma_{\mathrm{vac}} \\
\left(\mathrm{cm}^{-1}\right)\end{array}$} & \multirow{2}{*}{$\begin{array}{l}\Delta \sigma_{\text {vac }} \\
\left(\mathrm{cm}^{-1}\right)\end{array}$} & & & & & & & & \\
\hline & & & & & & Type & $p$ & $n$ & $g_{1}$ & $g_{2}$ & \\
\hline$M$ & 2504.275 & 39919.71 & -0.09 & $94^{\circ}-131$ & & & & & & & \\
\hline$M$ & 2499.679 & 39993.09 & +0.10 & $52^{\circ}-90$ & M & 6 & $(0.319)$ & & $(1.061)$ & 1.189 & \\
\hline$M$ & 2498.863 & 40006.06 & +0.05 & $130^{\circ}-129$ & $M$ & 4 & $(0)$ & 0.844 & $(1.018)$ & 1.069 & \\
\hline$M$ & 2498.402 & 40013.52 & -0.01 & $51^{\circ} .124$ & $M$ & 5 & $(0.104)$ & 1.623 & 0.88 & 1.09 & \\
\hline \multirow[t]{2}{*}{$M$} & 2495.353 & 40062.43 & +0.02 & $51^{\circ} \cdot 84$ & $M$ & 6 & $(0.813)$ & 1.022 & 0.860 & 1.183 & \\
\hline & & & -0.06 & $54^{\circ} \cdot 47$ & & & & & & & \\
\hline$M$ & 2489.64 & 40154.3 & -0.2 & $150^{\circ} \cdot 133$ & $M$ & 6 & $(0.989)$ & 1.197 & 1.091 & 1.314 & \\
\hline$K$ & 2486.133 & 40210.97 & -0.13 & $15^{\circ}-89$ & $M$ & 5 & $(0.322)$ & 2.112 & 0.502 & 1.146 & \\
\hline$M$ & 2485.058 & 40228.36 & 0.00 & $91^{\circ}-52$ & $M$ & 6 & $(0.534)$ & 1.322 & 1.219 & 1.429 & \\
\hline $\mathrm{K}$ & 2479.560 & 40317.57 & -0.10 & $51^{\circ}-125$ & & & & & & & \\
\hline$M$ & 2476.96 & 40359.9 & +0.1 & $86^{\circ}-14$ & $M$ & 5 & $(0.291)$ & 2.045 & 1.168 & 0.586 & \\
\hline$M$ & 2473.98 & 40408.5 & 0.0 & $51^{\circ}-126$ & & & & & & & \\
\hline$M$ & 2468.150 & 40503.93 & -0.02 & $126^{\circ}-91$ & $M$ & $7 b$ & $(0)$ & 1.236 & 1.236 & 1.236 & \\
\hline$M$ & 2466.126 & 40537.17 & +0.01 & $126^{\circ}-50$ & $M$ & 5 & & 1.374 & 1.223 & (1.163) & \\
\hline$M$ & 2464.844 & 40558.25 & 0.00 & $45^{\circ}-14$ & $M$ & 6 & $(1.085)$ & & 1.313 & 0.590 & \\
\hline$M$ & 2462.854 & 40591.02 & 0.00 & $44^{\circ}-49$ & $M$ & 4 & $(0.197)$ & 0.511 & 1.471 & 1.083 & \\
\hline$M$ & 2459.007 & 40654.52 & -0.02 & $85^{\circ}-13$ & $M$ & 5 & $(0.148)$ & 1.397 & 0.951 & 0.651 & \\
\hline$M$ & 2457.751 & 40675.29 & 0.00 & $88^{\circ}-91$ & $M$ & 5 & $(0.113)$ & 1.784 & 1.015 & 1.237 & \\
\hline$K$ & 2456.381 & 40697.97 & +0.14 & $86^{\circ}-49 ?$ & & & & & & & \\
\hline$M$ & 2456.292 & 40699.45 & +0.04 & $51^{\circ}-85$ & $M$ & 6 & $(0.465)$ & 0.756 & 0.849 & 0.663 & \\
\hline$M$ & 2455.746 & 40708.50 & 0.00 & $88^{\circ}-50$ & $M$ & 6 & $(0.400)$ & & 1.003 & $(1.163)$ & \\
\hline$M$ & 2448.700 & 40825.62 & 0.00 & $13^{\circ}-13$ & $M$ & & $(0.187)$ & & 0.265 & $(0.639)$ & \\
\hline$M$ & 2444.469 & 40896.28 & 0.00 & $45^{\circ}-49$ & $M$ & 4 & $(0.101)$ & 0.726 & 1.273 & 1.055 & \\
\hline$M$ & 2443.951 & 40904.95 & +0.14 & $51^{\circ}-127$ & $M$ & 5 & $(0.173)$ & 2.094 & 0.864 & 1.217 & \\
\hline$M$ & 2440.248 & 40967.04 & -0.10 & $52^{\circ}-131$ & $M$ & 5 & & 1.125 & $(1.061)$ & 1.079 & \\
\hline$M$ & 2432.85 & 41091.6 & +0.1 & $92^{\circ}-93$ & $M$ & 5 & $(0.114)$ & 1.776 & 0.963 & 1.199 & \\
\hline$M$ & 2431.16 & 41120.1 & -0.1 & $51^{\circ} .86$ & $M$ & 6 & $(0.547)$ & 0.970 & 0.86 & 1.08 & \\
\hline$M$ & 2423.675 & 41247.15 & +0.15 & $129^{\circ}-54$ & & & & & & & \\
\hline$v$ & 2422.26 & 41271.2 & 0.0 & $91^{\circ}-16$ & & & & & & & \\
\hline$v$ & 2418.07 & 41342.7 & +0.2 & $149^{\circ}-91$ & & & & & & & \\
\hline$M$ & 2413.405 & 41422.63 & +0.04 & $123^{\circ}-49$ & & & & & & & \\
\hline$M$ & 2407.581 & 41522.82 & 0.00 & $87^{\circ} \cdot 14$ & $M$ & & $(0.309)$ & & 1.201 & $(0.586)$ & \\
\hline$M$ & 2406.434 & 41542.65 & -0.03 & $127^{\circ}-91$ & $M$ & $6 ?$ & & 1.242 & 1.242 & 1.242 & \\
\hline
\end{tabular}


Table 6 (continued)

\begin{tabular}{|c|c|c|c|c|c|c|c|c|c|c|c|}
\hline \multirow{3}{*}{ Reference } & \multirow{3}{*}{$\begin{array}{c}\text { Wave } \\
\text { Length } \\
\lambda(\AA)\end{array}$} & \multicolumn{2}{|c|}{ Frequency } & \multirow{3}{*}{ Transition } & \multirow{3}{*}{ Reference } & \multirow{2}{*}{\multicolumn{5}{|c|}{ Observed Zeeman Pattern }} & \multirow{3}{*}{$\begin{array}{c}\sigma_{230}-\sigma_{232} \\
\left(\mathrm{~cm}^{-1}\right)\end{array}$} \\
\hline & & \multirow{2}{*}{$\begin{array}{c}\sigma_{\mathrm{vac}} \\
\left(\mathrm{cm}^{-1}\right)\end{array}$} & \multirow{2}{*}{$\begin{array}{l}\Delta \sigma_{\text {Vac }} \\
\left(\mathrm{cm}^{-1}\right)\end{array}$} & & & & & & & & \\
\hline & & & & & & Type & $p$ & $n$ & $g_{1}$ & $g_{2}$ & \\
\hline$M$ & 2404.510 & 41575.85 & -0.04 & $127^{\circ}-50$ & $M$ & 5 & & 1.475 & 1.252 & $(1.163)$ & \\
\hline$M$ & 2404.174 & 41581.69 & 0.00 & $94^{\circ} \cdot 132$ & $M$ & 6 & $(0.375)$ & 1.197 & 1.143 & 1.250 & \\
\hline$M$ & 2395.67 & 41729.3 & 0.0 & $50^{\circ}-18$ & $M$ & 5 & $(0.156)$ & $1.120^{\star}$ & & & \\
\hline$M$ & 2393.113 & 41773.84 & -0.05 & $51^{\circ}-41$ & $M$ & $7 b$ & & 0.826 & $(0.857)$ & 0.878 & \\
\hline $\mathrm{C}$ & 2388.1 & 41862 & 0 & $51^{\circ} \cdot 128 ?$ & & & & & & & \\
\hline$M$ & 2386.81 & 41884.1 & 0.0 & $14^{\circ}-14$ & $M$ & & $(0.818)$ & & 2.222 & $(0.586)$ & \\
\hline$\checkmark$ & 2384.36 & 41927.2 & +0.3 & $129^{\circ}-94 ?$ & & & & & & & \\
\hline$M$ & 2377.832 & 42042.27 & -0.04 & $90^{\circ}-50$ & $M$ & $7 b$ & $(0)$ & 1.157 & 1.157 & 1.157 & \\
\hline$M$ & 2375.075 & 42091.08 & -0.13 & $51^{\circ}-87$ & $M$ & 6 & $(0.424)$ & 0.982 & 0.896 & 1.068 & \\
\hline \multirow[t]{2}{*}{$M$} & 2373.846 & 42112.86 & -0.04 & $51^{\circ}-88$ & $M$ & 6 & $(0.639)$ & 1.000 & 0.862 & 1.118 & \\
\hline & & & +0.04 & $44^{\circ}-13$ & & & & & & & \\
\hline$M$ & 2367.821 & 42220.04 & +0.27 & $86^{\circ}-13$ & & & & & & & \\
\hline$M$ & 2366.984 & 42234.98 & +0.08 & $92^{\circ}-92$ & & & & & & & \\
\hline \multirow[t]{2}{*}{$v$} & 2366.02 & 42252.1 & +0.4 & $124^{\circ}-49 ?$ & & & & & & & \\
\hline & & & 0.0 & $125^{\circ}-49$ & & & & & & & \\
\hline $\mathrm{K}$ & 2362.750 & 42310.62 & +0.19 & $48^{\circ}-50 ?$ & & & & & & & \\
\hline$M$ & 2356.755 & 42418.25 & +0.06 & $45^{\circ}-13$ & $M$ & 6 & $(1.003)$ & 0.990 & 1.308 & 0.640 & \\
\hline$M$ & 2355.251 & 42445.32 & -0.03 & $51^{\circ}-42$ & $M$ & $7 b$ & & 0.761 & $(0.857)$ & 0.921 & \\
\hline$M$ & 2354.02 & 42467.5 & 0.0 & $49^{\circ}-52$ & $M$ & 5 & $(0.244)$ & 2.186 & 0.933 & 1.432 & \\
\hline $\mathrm{K}$ & 2349.69 & 42545.8 & -0.1 & $92^{\circ}-53$ & & & & & & & \\
\hline$v$ & 2333.87 & 42834.1 & -0.1 & $51^{\circ}-90$ & & & & & & & \\
\hline $\mathrm{K}$ & 2331.065 & 42885.67 & -0.10 & $92^{\circ} .52$ & & & & & & & \\
\hline$M$ & 2329.064 & 42922.51 & -0.24 & $49^{\circ}-51$ & $M$ & & $(0)$ & 1.074 & 0.927 & $(0.986)$ & \\
\hline$M$ & 2326.922 & 42962.02 & +0.10 & $88^{\circ}-14$ & $M$ & 5 & $(0.206)$ & 1.618 & 1.003 & 0.591 & \\
\hline$M$ & 2317.926 & 43128.73 & +0.12 & $126^{\circ}-49$ & & & & & & & \\
\hline $\mathrm{K}$ & 2310.88 & 43260.2 & -0.1 & $130^{\circ}-132$ & & & & & & & \\
\hline V & 2305.46 & 43361.9 & +0.4 & $89^{\circ}-14 ?$ & & & & & & & \\
\hline
\end{tabular}

* These data are inconsistent with the elassifications listed. 
Table 6 (continued)

\begin{tabular}{|c|c|c|c|c|c|c|c|c|c|c|c|}
\hline \multirow{3}{*}{ Reference } & \multirow{3}{*}{$\begin{array}{c}\text { Wave } \\
\text { Length } \\
\lambda(\AA)\end{array}$} & \multicolumn{2}{|c|}{ Frequency } & \multirow{3}{*}{ Transition } & \multirow{3}{*}{ Reference } & \multirow{2}{*}{\multicolumn{5}{|c|}{ Observed Zeeman Pattern }} & \multirow{3}{*}{$\begin{array}{c}\sigma_{230}-\sigma_{232} \\
\left(\mathrm{~cm}^{-1}\right)\end{array}$} \\
\hline & & \multirow{2}{*}{$\begin{array}{c}\sigma_{v a c} \\
\left(\mathrm{~cm}^{-1}\right)\end{array}$} & \multirow{2}{*}{$\begin{array}{l}\Delta \sigma_{\mathrm{Vac}} \\
\left(\mathrm{cm}^{-1}\right)\end{array}$} & & & & & & & & \\
\hline & & & & & & Type & $p$ & $n$ & $g_{1}$ & $g_{2}$ & \\
\hline V & 2287.65 & 43699.5 & -0.1 & $89^{\circ} \cdot 49$ & & & & & & & \\
\hline$M$ & 2280.433 & 43837.76 & -0.14 & $47^{\circ}-14$ & $M$ & 6 & $(0.862)$ & & 1.161 & $(0.586)$ & \\
\hline$M$ & 2263.40 & 44167.6 & +0.3 & $127^{\circ}-49 ?$ & & & & & & & \\
\hline$M$ & 2243.26 & 44564.1 & +0.3 & $48^{\circ}-14 ?$ & & & & & & & \\
\hline$M$ & 2239.757 & 44633.81 & +0.05 & $90^{\circ} .49$ & $M$ & 6 & $(0.164)$ & & 1.142 & 1.076 & \\
\hline$M$ & 2230.35 & 44822.0 & +0.2 & $88^{\circ}-13$ & $M$ & 5 & $(0.180)$ & 1.531 & 1.003 & 0.651 & \\
\hline$M$ & 2226.38 & 44902.0 & +0.1 & $48^{\circ}-49$ & & & & & & & \\
\hline$M$ & 2212.87 & 45176.1 & 0.0 & $91^{\circ}-50$ & & & & & & & \\
\hline$M$ & 2210.64 & 45221.6 & +0.2 & $89^{\circ}-13$ & & & & & & & \\
\hline$M$ & 2197.63 & 45489.3 & +0.5 & $50^{\circ}-17 ?$ & & & & & & & \\
\hline$M$ & 2187.60 & 45697.9 & 0.0 & $47^{\circ} \cdot 13$ & & & & & & & \\
\hline$M$ & 2153.37 & 46424.2 & +0.4 & $48^{\circ}-13 ?$ & & & & & & & \\
\hline
\end{tabular}


Table 7. Zeeman Data of Unclassified Th II Lines

\begin{tabular}{|c|c|c|c|c|c|c|c|c|}
\hline$\lambda(\stackrel{\circ}{)}$ & $\begin{array}{c}\text { Type } \\
\text { of Pattern }\end{array}$ & $\begin{array}{l}\text { Separation } \\
\text { (Lorentz units) }\end{array}$ & $\begin{array}{c}p \\
\text { (Lorentz } \\
\text { units) }\end{array}$ & $\begin{array}{c}n \\
\text { (Lorentz } \\
\text { units) }\end{array}$ & $J_{1}$ & $J_{2}$ & $g_{1}$ & $g_{2}$ \\
\hline 6378.76 & 6 & 0.184 & $(0.455)$ & 0.974 & $21 / 2$ & $21 / 2$ & 1.064 & 0.882 \\
\hline 6369.144 & 6 & 0.062 & $(0.031)$ & 1.029 & $1 / 2$ & $1 / 2$ & 1.060 & 0.998 \\
\hline 6289.490 & 5 & 0.154 & $(0.075)$ & 1.663 & $41 / 2$ & $31 / 2$ & 1.125 & 0.971 \\
\hline $6066.50 \mathrm{I}$ & 4 & 0.166 & $(0.083)$ & 0.654 & $21 / 2$ & $11 / 2$ & 0.903 & 1.069 \\
\hline 6061.48 & 6 & 0.084 & $(0.042)$ & 0.939 & $1 / 2$ & $1 / 2$ & 0.897 & 0.981 \\
\hline 6037.692 & 6 & 0.076 & $(0.038)$ & 0.711 & $1 / 2$ & $1 / 2$ & 0.749 & 0.673 \\
\hline 6034.50 & 4 & 0.211 & $(0.099)$ & 0.949 & $21 / 2$ & $11 / 2$ & 1.266 & 1.477 \\
\hline 6000.88 & 4 & 0.155 & $(0.081)$ & 1.532 & $1 \frac{1}{2}$ & $1 / 2$ & 1.610 & 1.765 \\
\hline 5973.669 & 4 & 0.172 & $(0.086)$ & 1.078 & $21 / 2$ & $11 / 2$ & 1.341 & 1.513 \\
\hline 5910.472 & 6 & 0.106 & $(0.053)$ & 1.113 & $1 / 2$ & $1 / 2$ & 1.166 & 1.060 \\
\hline 5194.22 & 5 & 0.285 & & 1.042 & $2 \frac{1}{2}$ & $11 / 2$ & 0.610 & 0.325 \\
\hline 5178.24 & 6 & 0.333 & & 0.460 & $11 / 2$ & $11 / 2$ & 0.624 & 0.291 \\
\hline 4703.992 & 6 & 0.290 & $(0.145)$ & 0.666 & & & & \\
\hline 4666.003 & 4 & 0.245 & $(0.119)$ & 0.120 & $21 / 2$ & $1 / 2$ & 0.472 & 0.717 \\
\hline 4619.562 & 5 & 0.122 & $(0.061)$ & 1.270 & $31 / 2$ & $2 \frac{1}{2}$ & 0.965 & 0.843 \\
\hline 4619.472 & 4 & 0.126 & & 0.649 & $4 \frac{1}{2}$ & $31 / 2$ & 1.083 & 1.207 \\
\hline 4592.79 & 6 & & & & $11 / 2$ & $11 / 2$ & & \\
\hline 4592.668 & 4 or 5 & 0.290 & $(0.145)$ & & & & & \\
\hline 4571.948 & 6 & 0.090 & & 0.796 & $2 \frac{1}{2}$ & $2 \frac{1}{2}$ & 0.753 & 0.833 \\
\hline 4562.374 & 5 & 0.120 & $(0.060)$ & 1.219 & $41 / 2$ & $31 / 2$ & 0.799 & 0.679 \\
\hline 4555.815 & 4 or 5 & 0.178 & $(0.089)$ & & & & & \\
\hline 4513.827 & 4 or 5 & 0.293 & $(0.146)$ & & & & & \\
\hline 4505.226 & 5 & 0.215 & $(0.112)$ & 1.948 & $31 / 2$ & $2 \frac{1}{2}$ & 1.388 & 1.164 \\
\hline 4500.04 & 5 & 0.198 & & & & & & \\
\hline 4457.931 & 6 & 0.272 & & 1.388 & $21 / 2$ & $2 \frac{1}{2}$ & 1.525 & 1.253 \\
\hline 4443.099 & 6 & & $(0.652)$ & & $31 / 2$ & $31 / 2$ & & \\
\hline 4397.919 & 4 or 5 & 0.106 & $(0.048)$ & & $4 \frac{1}{2}$ & $31 / 2$ & & \\
\hline 4342.273 & 6 & 0.136 & & & $4 \frac{1}{2}$ & $4 \frac{1}{2}$ & & \\
\hline 4341.032 & 4 & 0.087 & $(0.037)$ & 0.721 & $31 / 2$ & $21 / 2$ & 0.939 & 1.026 \\
\hline 4335.403 & 6 & 0.096 & & & $21 / 2$ & $21 / 2$ & & \\
\hline 4333.942 & 6 & 0.090 & $(0.674)$ & & $71 / 2$ & $71 / 2$ & & \\
\hline 4313.314 & 4 & 0.218 & $(0.109$ & 0.172 & $21 / 2$ & $11 / 2$ & 0.499 & 0.717 \\
\hline 4309.994 & 6 & 0.454 & $(0.227)$ & 1.116 & $1 / 2$ & $1 / 2$ & 1.343 & 0.889 \\
\hline 4308.085 & 4 or 5 & 0.090 & $(0.045)$ & & $31 / 2$ & $2 \frac{1}{2}$ & & \\
\hline 4289.926 & 6 & 0.103 & $(0.360)$ & 0.876 & $31 / 2$ & $31 / 2$ & 0.932 & 0.829 \\
\hline 4281.37 & 5 & 0.406 & $(0.203)$ & 1.335 & $11 / 2$ & $1 / 2$ & 1.132 & 0.726 \\
\hline 4281.119 & 6 & & & & $2 \frac{1}{2}$ & $2 \frac{1}{2}$ & & \\
\hline 4265.942 & 6 & & & & $11 / 2$ & $11 / 2$ & & \\
\hline 4260.45 & 5 & 0.193 & $(0.097)$ & 2.438 & $51 / 2$ & $4 \frac{1}{2}$ & 1.565 & 1.374 \\
\hline 4239.889 & 5 & 0.276 & $(0.136)$ & 1.911 & $31 / 2$ & $2 \frac{1}{2}$ & 1.220 & 0.944 \\
\hline 4199.022 & 5 & 0.306 & $(0.154)$ & 2.264 & $31 / 2$ & $2 \frac{1}{2}$ & 1.499 & 1.193 \\
\hline 4195.782 & 5 & 0.143 & $(0.071)$ & 1.413 & $21 / 2$ & $1 \frac{1}{2}$ & 1.199 & 1.056 \\
\hline 4195.561 & 6 & 0.138 & $(0.068)$ & 1.122 & $1 / 2$ & $1 / 2$ & 1.191 & 1.054 \\
\hline 4171.349 & 6 & 0.390 & $(0.195)$ & 1.189 & $1 / 2$ & $1 / 2$ & 1.384 & 0.994 \\
\hline 4158.567 & 6 & 0.283 & $(1.285)$ & & $41 / 2$ & $4 \frac{1}{2}$ & & \\
\hline
\end{tabular}


Table 7 (continued)

\begin{tabular}{|c|c|c|c|c|c|c|c|c|}
\hline$\lambda(\stackrel{\circ}{\mathrm{A}})$ & $\begin{array}{l}\text { Type } \\
\text { of Pattern }\end{array}$ & $\begin{array}{c}\text { Separation } \\
\text { (Lorentz units) }\end{array}$ & $\begin{array}{c}p \\
\text { (Lorentz } \\
\text { units) }\end{array}$ & $\begin{array}{c}n \\
\text { (Lorentz } \\
\text { units) }\end{array}$ & $J_{1}$ & $J_{2}$ & $g_{1}$ & $g_{2}$ \\
\hline 4146.193 & 4 or 5 & 0.099 & $(0.048)$ & & $31 / 2$ & $2 \frac{1}{2}$ & & \\
\hline 4140.239 & 6 & 0.418 & $(0.209)$ & 1.047 & $1 / 2$ & $1 / 2$ & 1.256 & 0.838 \\
\hline 4134.117 & 5 & 0.222 & $(0.111)$ & 1.937 & $51 / 2$ & $41 / 2$ & 0.938 & 0.716 \\
\hline 4132.751 & 6 & 0.338 & $(0.169)$ & 0.973 & $1 / 2$ & $1 / 2$ & 1.142 & 0.804 \\
\hline 4122.67 & 6 & 0.177 & $(0.789)$ & & $41 / 2$ & $4 \frac{1}{2}$ & & \\
\hline 4120.873 & 6 & 0.064 & $(0.284)$ & & $4 \frac{1}{2}$ & $4 \frac{1}{2}$ & & \\
\hline 4073.775 & 6 & 0.330 & $(0.165)$ & 1.123 & $1 / 2$ & $1 / 2$ & 1.288 & 0.958 \\
\hline 4064.416 & 4 or 5 & 0.115 & $(0.058)$ & & $4 \frac{1}{2}$ & $31 / 2$ & & \\
\hline 4062.264 & 6 & 0.094 & $(0.328)$ & & $31 / 2$ & $31 / 2$ & & \\
\hline 4042.623 & 4 or 5 & 0.122 & $(0.061)$ & & $41 / 2$ & $3 \frac{1}{2}$ & & \\
\hline 4041.207 & 4 & 0.334 & $(0.168)$ & 0.975 & $11 / 2$ & $1 / 2$ & 1.143 & 1.477 \\
\hline 4034.96 & 4 or 5 & 0.173 & $(0.084)$ & & $31 / 2$ & $2 \frac{1}{2}$ & & \\
\hline 4032.54 & 6 & 0.300 & $(0.150)$ & 0.881 & $1 / 2$ & $1 / 2$ & 1.031 & 0.731 \\
\hline 3996.066 & 5 & 0.088 & $(0.045)$ & 1.469 & $51 / 2$ & $4 \frac{1}{2}$ & 1.064 & 0.976 \\
\hline 3994.56 & 6 & & $(0.041)$ & 0.680 & & & & \\
\hline 3991.701 & 4 or 5 & 0.191 & $(0.102)$ & & $31 / 2$ & $2 \frac{1}{2}$ & & \\
\hline 3979.063 & 6 & 0.186 & $(0.832)$ & 0.871 & $4 \frac{1}{2}$ & $4 \frac{1}{2}$ & 0.964 & 0.781 \\
\hline 3951.68 & 5 & 0.191 & $(0.092)$ & 1.933 & $31 / 2$ & $2 \frac{1}{2}$ & 1.446 & 1.255 \\
\hline 3948.971 & 6 & 0.144 & $(0.072)$ & 1.079 & $1 / 2$ & $1 / 2$ & 1.151 & 1.007 \\
\hline 3947.315 & 4 or 5 & 0.148 & $(0.072)$ & & $4 \frac{1}{2}$ & $31 / 2$ & & \\
\hline 3932.90 & 4 or 5 & 0.190 & $(0.095)$ & & $41 / 2$ & $31 / 2$ & & \\
\hline 3920.399 & 5 & 0.251 & $(0.122)$ & 2.038 & $31 / 2$ & $2 \frac{1}{2}$ & 1.428 & 1.177 \\
\hline 3905.262 & 5 & & & & $4 \frac{1}{2}$ & $31 / 2$ & & \\
\hline 3903.206 & 4 or 5 & 0.250 & $(0.127)$ & & $4 \frac{1}{2}$ & $31 / 2$ & & \\
\hline 3895.42 & 6 & & $(0.327)$ & & $4 \frac{1}{2}$ & $4 \frac{1}{2}$ & 0.863 & 0.936 \\
\hline 3879.66 & 4 & 0.070 & $(0.035)$ & 0.956 & $31 / 2$ & $21 / 2$ & 1.131 & 1.201 \\
\hline 3862.501 & 4 or 5 & 0.127 & $(0.052)$ & & $4 \frac{1}{2}$ & $31 / 2$ & & \\
\hline 3830.888 & 6 & 0.282 & $(0.671)$ & 1.585 & $2 \frac{1}{2}$ & $2 \frac{1}{2}$ & 1.719 & 1.451 \\
\hline 3818.485 & 4 or 5 & 0.264 & $(0.129)$ & & $31 / 2$ & $21 / 2$ & & \\
\hline 3788.443 & 4 or 5 & 0.366 & $(0.183)$ & & $2 \frac{1}{2}$ & $11 / 2$ & & \\
\hline 3776.297 & 4 or 5 & 0.167 & $(0.074)$ & & $31 / 2$ & $2 \frac{1}{2}$ & & \\
\hline 3767.905 & 4 & 0.182 & $(0.098)$ & 1.013 & $21 / 2$ & $1 \frac{1}{2}$ & 1.286 & 1.468 \\
\hline 3743.004 & 6 & & $(0.144)$ & 1.193 & $1 / 2$ & $1 / 2$ & 1.337 & 1.049 \\
\hline 3736.949 & 4 & 0.666 & $(0.335)$ & 1.004 & $11 / 2$ & $1 / 2$ & 1.339 & 2.005 \\
\hline 3724.739 & 5 & 0.158 & $(0.079)$ & 1.733 & $31 / 2$ & $2 \frac{1}{2}$ & 1.338 & 1.180 \\
\hline 3723.175 & 4 or 5 & 0.164 & $(0.082)$ & & $41 / 2$ & $31 / 2$ & & \\
\hline 3708.851 & 6 & 0.123 & $(0.416)$ & & $31 / 2$ & $31 / 2$ & & \\
\hline 3702.950 & 6 & & $(0.114)$ & 1.147 & $1 / 2$ & $1 / 2$ & 1.261 & 1.033 \\
\hline 3693.94 & 6 & 0.158 & $(0.720)$ & 1.092 & $41 / 2$ & $41 / 2$ & 1.172 & 1.012 \\
\hline 3688.763 & 5 & 0.090 & $(0.046)$ & 1.518 & $31 / 2$ & $21 / 2$ & 1.293 & 1.203 \\
\hline 3681.252 & 4 or 5 & 0.323 & $(0.160)$ & & $31 / 2$ & $2 \frac{1}{2}$ & & \\
\hline 3672.362 & 6 & 0.154 & $(0.386)$ & & $21 / 2$ & $2 \frac{1}{2}$ & & \\
\hline 3652.168 & 6 & 0.120 & $(0.603)$ & 1.125 & $51 / 2$ & $51 / 2$ & 1.180 & 1.070 \\
\hline 3635.944 & 4 & 0.125 & $(0.061)$ & 0.608 & $31 / 2$ & $2 \frac{1}{2}$ & 0.913 & 1.038 \\
\hline 3634.221 & 4 & & $(0.046)$ & 0.637 & & & & \\
\hline
\end{tabular}


Table 7 (continued)

\begin{tabular}{|c|c|c|c|c|c|c|c|c|}
\hline$\lambda(\stackrel{\circ}{\AA})$ & $\begin{array}{l}\text { Type } \\
\text { of Pattern }\end{array}$ & $\begin{array}{c}\text { Separation } \\
\text { (Lorentz units) }\end{array}$ & $\begin{array}{c}p \\
\text { (Lorentz } \\
\text { units) }\end{array}$ & $\begin{array}{c}n \\
\text { (Lorentz } \\
\text { units) }\end{array}$ & $J_{1}$ & $J_{2}$ & $g_{1}$ & $g_{2}$ \\
\hline 3614.011 & 6 & 0.102 & $(0.356)$ & 1.196 & $31 / 2$ & $31 / 2$ & 1.247 & 1.145 \\
\hline 3610.039 & 4 & 0.277 & $(0.138)$ & 0.749 & $21 / 2$ & $1 \frac{1}{2}$ & 1.165 & 1.442 \\
\hline 3592.776 & 6 & 0.158 & $(0.389)$ & 0.903 & $2 \frac{1}{2}$ & $2 \frac{1}{2}$ & 0.981 & 0.825 \\
\hline 3582.040 & 6 & & $(0.240)$ & 1.126 & $1 / 2$ & $1 / 2$ & 1.366 & 0.886 \\
\hline 3553.124 & 6 & 0.358 & $(0.179)$ & 1.108 & $1 / 2$ & $1 / 2$ & 1.287 & 0.929 \\
\hline 3538.223 & 6 & 0.492 & $(0.245)$ & 0.833 & $1 / 2$ & $1 / 2$ & 1.079 & 0.587 \\
\hline 3498.625 & 6 & & $(0.300)$ & & & & & \\
\hline 3486.521 & 6 & 0.420 & $(0.210)$ & 1.087 & $1 / 2$ & $1 / 2$ & 1.297 & 0.877 \\
\hline 3456.456 & 4 or 5 & 0.424 & $(0.209)$ & & $21 / 2$ & $11 / 2$ & & \\
\hline 3421.204 & 4 & 0.114 & $(0.060)$ & 0.644 & $41 / 2$ & $31 / 2$ & 1.064 & 1.178 \\
\hline 3421.234 & 6 & 0.061 & $(0.392)$ & 1.197 & $51 / 2$ & $51 / 2$ & 1.233 & 1.165 \\
\hline 3419.224 & 6 & 0.107 & $(0.701)$ & 1.116 & $71 / 2$ & $71 / 2$ & 1.169 & 1.063 \\
\hline 3416.440 & 6 & 0.122 & $(0.183)$ & & $11 / 2$ & $11 / 2$ & & \\
\hline 3411.362 & 5 & 0.291 & $(0.146)$ & 2.425 & $31 / 2$ & $21 / 2$ & 1.698 & 1.407 \\
\hline 3407.653 & 4 & 0.234 & $(0.121)$ & 0.330 & $4 \frac{1}{2}$ & $31 / 2$ & 1.149 & 1.383 \\
\hline 3402.721 & 6 & 0.155 & $(0.859)$ & 1.524 & $51 / 2$ & $51 / 2$ & 1.602 & 1.446 \\
\hline 3398.564 & 6 & & $(0.089)$ & 1.101 & $1 / 2$ & $1 / 2$ & 1.190 & 1.012 \\
\hline 3373.499 & 4 & 0.133 & $(0.070)$ & 0.828 & $31 / 2$ & $2 \frac{1}{2}$ & 1.161 & 1.294 \\
\hline 3364.698 & 6 & 0.156 & $(0.687)$ & 1.318 & $4 \frac{1}{2}$ & $41 / 2$ & 1.240 & 1.395 \\
\hline 3343.271 & 4 & 0.300 & $(0.165)$ & 0.667 & $3 \frac{1}{2}$ & $21 / 2$ & 1.417 & 1.717 \\
\hline 3332.47 & 5 & 0.209 & $(0.109)$ & 1.573 & $21 / 2$ & $11 / 2$ & 1.259 & 1.050 \\
\hline 3329.719 & 6 & 0.170 & $(0.622)$ & & $3 \frac{1}{2}$ & $31 / 2$ & & \\
\hline 3322.47 & 5 & & $\langle 0.115\rangle$ & & $2 \frac{1}{2}$ & $11 / 2$ & & \\
\hline 3301.716 & 5 & 0.136 & $(0.068)$ & & $31 / 2$ & $2 \frac{1}{2}$ & & \\
\hline 3282.969 & 4 & 0.127 & $(0.071)$ & 0.696 & $4 \frac{1}{2}$ & $31 / 2$ & 1.141 & 1.268 \\
\hline 3232.74 & 4 or 5 & 0.172 & $(0.086)$ & & $21 / 2$ & $11 / 2$ & & \\
\hline 3229.99 & 6 & 0.270 & $(0.404)$ & & $1 \frac{1}{2}$ & $11 / 2$ & & \\
\hline 3226.412 & 5 & 0.504 & $(0.252)$ & 2.327 & $21 / 2$ & $11 / 2$ & & \\
\hline 3225.616 & 4 & 0.191 & $(0.083)$ & 0.893 & $3 \frac{1}{2}$ & $21 / 2$ & 1.343 & 1.523 \\
\hline 3221.88 & 4 or 5 & 0.152 & $(0.076)$ & & $51 / 2$ & $4 \frac{1}{2}$ & & \\
\hline 3214.416 & 6 & 0.130 & $(0.443)$ & & $31 / 2$ & $31 / 2$ & & \\
\hline 3207.677 & 4 or 5 & 0.256 & $(0.127)$ & & $3 \frac{1}{2}$ & $2 \frac{1}{2}$ & & \\
\hline 3206.275 & 4 & 0.234 & $(0.108)$ & 0.969 & $31 / 2$ & $21 / 2$ & 1.554 & 1.788 \\
\hline 3205.281 & 6 & & $(0.450)$ & 1.981 & $1 / 2$ & $1 / 2$ & 2.431 & 1.531 \\
\hline 3202.132 & 4 or 5 & 0.218 & $(0.109)$ & & $3 \frac{1}{2}$ & $21 / 2$ & & \\
\hline 3195.326 & 4 & 0.166 & $(0.088)$ & 0.381 & $31 / 2$ & $2 \frac{1}{2}$ & 0.796 & 0.962 \\
\hline 3190.11 & 6 & 0.166 & $(0.755)$ & 0.933 & $4 \frac{1}{2}$ & $41 / 2$ & 1.017 & 0.849 \\
\hline 3187.023 & 6 & 0.177 & $(0.629)$ & & $31 / 2$ & $31 / 2$ & & \\
\hline 3165.71 & 5 & 0.244 & $(0.128)$ & 1.602 & $21 / 2$ & $11 / 2$ & 1.236 & 0.992 \\
\hline 3163.920 & 6 & 0.095 & $(0.237)$ & & $2 \frac{1}{2}$ & $2 \frac{1}{2}$ & & \\
\hline 3160.781 & 6 & 0.169 & $(0.590)$ & & $31 / 2$ & $31 / 2$ & & \\
\hline 3160.448 & 6 & 0.263 & $(0.922)$ & & $31 / 2$ & $31 / 2$ & & \\
\hline 3157.90 & 6 & 0.146 & $(0.073)$ & 1.171 & $1 / 2$ & $1 / 2$ & 1.244 & 1.098 \\
\hline 3155.014 & 4 or 5 & 0.124 & $(0.058)$ & & $31 / 2$ & $21 / 2$ & & \\
\hline 3154.594 & 4 or 5 & 0.114 & $(0.052)$ & & $2 \frac{1}{2}$ & $1 \frac{1}{2}$ & & \\
\hline
\end{tabular}


Table 7 (continued)

\begin{tabular}{|c|c|c|c|c|c|c|c|c|}
\hline$\lambda(\stackrel{\circ}{\mathrm{A}})$ & $\begin{array}{c}\text { Type } \\
\text { of Pattern }\end{array}$ & $\begin{array}{c}\text { Separation } \\
\text { (Lorentz units) }\end{array}$ & $\begin{array}{c}p \\
\text { (Lorentz } \\
\text { units) }\end{array}$ & $\begin{array}{c}n \\
\text { (Lorentz } \\
\text { units) }\end{array}$ & $J_{1}$ & $J_{2}$ & $g_{1}$ & $g_{2}$ \\
\hline 3150.457 & 6 & 0.108 & $(0.489)$ & 1.829 & $4 \frac{1}{2}$ & $4 \frac{1}{2}$ & 1.883 & 1.775 \\
\hline 3122.37 & 6 & 0.263 & $(0.395)$ & & $11 / 2$ & $11 / 2$ & & \\
\hline 3099.109 & 5 & 0.465 & $(0.226)$ & 1.948 & $21 / 2$ & $11 / 2$ & 1.251 & 0.786 \\
\hline 3095.764 & 6 & 0.272 & $(0.406)$ & 1.625 & $1 \frac{1}{2}$ & $11 / 2$ & 1.761 & 1.489 \\
\hline 3094.74 & 6 & 0.214 & $(0.756)$ & 1.028 & $31 / 2$ & $31 / 2$ & 1.135 & 0.921 \\
\hline 3075.876 & 6 & 0.242 & $(0.844)$ & 1.231 & $31 / 2$ & $31 / 2$ & 1.352 & 1.111 \\
\hline 3075.668 & 6 & 0.235 & $(0.353)$ & & $11 / 2$ & $11 / 2$ & & \\
\hline 3050.841 & 6 & 0.144 & $(0.373)$ & 1.360 & $2 \frac{1}{2}$ & $2 \frac{1}{2}$ & 1.432 & 1.288 \\
\hline 3031.291 & 5 & 0.171 & $(0.088)$ & 1.704 & $2 \frac{1}{2}$ & $11 / 2$ & 1.448 & 1.277 \\
\hline 3026.131 & 6 & & $(0.064)$ & 1.061 & & & & \\
\hline 3025.435 & 4 & 0.572 & $(0.286)$ & 0.815 & $21 / 2$ & $11 / 2$ & 1.673 & 2.245 \\
\hline 3023.56 & 6 & & $(0.361)$ & 0.616 & $1 / 2$ & $1 / 2$ & 0.979 & 0.253 \\
\hline 3021.46 & 4 & 0.122 & $(0.055)$ & 0.512 & $51 / 2$ & $4 \frac{1}{2}$ & 1.061 & 1.183 \\
\hline 3005.575 & & 0.206 & $(0.103)$ & & $41 / 2$ & $31 / 2$ & & \\
\hline 2998.329 & 5 & 0.306 & $(0.153)$ & 2.092 & $2 \frac{1}{2}$ & $1 \frac{1}{2}$ & 1.633 & 1.327 \\
\hline 2977.225 & 6 & 0.169 & $(0.425)$ & 1.189 & $21 / 2$ & $23 / 2$ & 1.274 & 1.104 \\
\hline 2959.91 & 6 & & $(0.565)$ & & $2 / / 2$ & $2 \frac{1}{2}$ & 1.344 & 1.118 \\
\hline 2944.985 & 6 & 0.278 & $(0.686)$ & 1.202 & $2 \frac{1}{2}$ & $2 \frac{1}{2}$ & 1.341 & 1.063 \\
\hline 2938.393 & 4 or 5 & 0.266 & $(0.128)$ & & $41 / 2$ & $31 / 2$ & & \\
\hline 2934.141 & 5 & 0.177 & $(0.083)$ & 1.888 & $4 \frac{1}{2}$ & $31 / 2$ & 1.269 & 1.092 \\
\hline 2930.913 & 6 & & $(0.082)$ & 1.004 & & & & \\
\hline 2916.44 & 4 or 5 & & $(0.086)$ & & $31 / 2$ & $2 \frac{1}{2}$ & & \\
\hline 2915.54 & 4 or 5 & 0.116 & & & $41 / 2$ & $31 / 2$ & & \\
\hline 2878.638 & 4 & 0.293 & $(0.147)$ & 0.614 & $2 \frac{1}{2}$ & $1 \frac{1}{2}$ & 1.054 & 1.347 \\
\hline 2852.502 & 5 & 0.255 & $(0.123)$ & 2.061 & $4 \frac{1}{2}$ & $31 / 2$ & 1.169 & 0.914 \\
\hline 2848.01 & 6 & 0.254 & $(0.631)$ & 1.370 & $21 / 2$ & $21 / 2$ & 1.497 & 1.243 \\
\hline 2814.574 & 4 & 0.188 & $(0.094)$ & 0.883 & $31 / 2$ & $2 \frac{1}{2}$ & 1.358 & 1.546 \\
\hline 2782.421 & 6 & & $(0.158)$ & 1.264 & $1 / 2$ & $1 / 2$ & 1.422 & 1.106 \\
\hline 2753.100 & 5 & 0.260 & $(0.127)$ & 1.107 & $11 / 2$ & $1 / 2$ & 0.977 & 0.717 \\
\hline 2735.49 & 6 & 0.169 & $(0.423)$ & & $2 \frac{1}{2}$ & $21 / 2$ & & \\
\hline 2731.583 & 4 or 5 & 0.389 & $(0.195)$ & & $31 / 2$ & $21 / 2$ & & \\
\hline 2728.70 & 5 & 0.634 & $(0.317)$ & 1.546 & $11 / 2$ & $1 / 2$ & 1.229 & 0.595 \\
\hline 2723.323 & 4 & 0.164 & $(0.081)$ & 0.922 & $31 / 2$ & $21 / 2$ & 1.332 & 1.496 \\
\hline 2718.798 & 6 & 0.370 & $(0.556)$ & & $11 / 2$ & $11 / 2$ & & \\
\hline 2717.265 & 4 & 0.231 & $(0.114)$ & 0.795 & $2 \frac{1}{2}$ & $11 / 2$ & 1.142 & 1.373 \\
\hline 2715.092 & 6 & 0.137 & & & $4 \frac{1}{2}$ & $4 \frac{1}{2}$ & & \\
\hline 2709.857 & 6 & 0.216 & $(0.756)$ & & $31 / 2$ & $31 / 2$ & & \\
\hline 2709.386 & 4 or 5 & 0.254 & $(0.137)$ & & $31 / 2$ & $2 \frac{1}{2}$ & & \\
\hline 2701.86 & 5 & 0.051 & $(0.024)$ & 1.172 & $1 \frac{1}{2}$ & $1 / 2$ & 1.147 & 1.096 \\
\hline 2695.207 & 4 & 0.154 & $(0.078)$ & 0.562 & $21 / 2$ & $11 / 2$ & 0.793 & 0.947 \\
\hline 2685.55 & 4 or 5 & 0.223 & $(0.105)$ & & $61 / 2$ & $51 / 2$ & & \\
\hline 2677.531 & 6 & 0.333 & $(1.166)$ & 1.163 & $31 / 2$ & $31 / 2$ & 1.330 & 0.997 \\
\hline 2673.922 & 6 & & $(0.359)$ & 0.865 & & & & \\
\hline 2671.458 & 4 & 0.141 & $(0.070)$ & 0.783 & $31 / 2$ & $2 \frac{1}{2}$ & 1.136 & 1.277 \\
\hline 2669.44 & 6 & 0.112 & $(0.056)$ & 1.168 & $1 / 2$ & $1 / 2$ & 1.224 & 1.112 \\
\hline
\end{tabular}


Table 7 (continued)

\begin{tabular}{|c|c|c|c|c|c|c|c|c|}
\hline$\lambda(\AA)$ & $\begin{array}{c}\text { Type } \\
\text { of Pattern }\end{array}$ & $\begin{array}{c}\text { Separation } \\
\text { (Lorentz units) }\end{array}$ & $\begin{array}{c}p \\
\text { (Lorentz } \\
\text { units) }\end{array}$ & $\begin{array}{c}n \\
\text { (Lorentz } \\
\text { units) }\end{array}$ & $J_{1}$ & $J_{2}$ & $8_{1}$ & $g_{2}$ \\
\hline 2667.07 & 6 & & $(0.731)$ & 0.967 & $1 / 2$ & $1 / 2$ & 1.698 & 0.236 \\
\hline 2666.87 & 4 & 0.207 & & 0.986 & $11 / 2$ & $1 / 2$ & 1.090 & 1.297 \\
\hline 2653.33 & 6 & 0.208 & & 0.910 & $3 \frac{1}{2}$ & $3 \frac{1}{2}$ & 1.014 & 0.806 \\
\hline 2647.50 & 6 & 1.369 & $(0.685)$ & 0.937 & $1 / 2$ & $1 / 2$ & 1.622 & 0.252 \\
\hline 2644.679 & 4 & 0.402 & $(0.201)$ & 1.222 & $1 / 2$ & $1 / 2$ & 1.423 & 1.825 \\
\hline 2642.615 & 4 & 0.223 & $(0.110)$ & 0.447 & $31 / 2$ & $21 / 2$ & 1.005 & 1.228 \\
\hline 2635.42 & 6 & & $(0.130)$ & 1.087 & & & & \\
\hline 2635.28 & 5 & 0.219 & $(0.109)$ & 1.631 & $31 / 2$ & $21 / 2$ & 1.084 & 0.865 \\
\hline 2631.048 & 4 or 5 & 0.206 & $(0.104)$ & & $21 / 2$ & $1 / \frac{2}{2}$ & & \\
\hline 2628.37 & 6 & & $(0.899)$ & 1.132 & & & & \\
\hline 2617.83 & 4 & 0.582 & $(0.291)$ & 1.001 & $11 / 2$ & $1 / 2$ & 1.292 & 1.874 \\
\hline 2615.353 & 6 & & $(0.080)$ & 1.110 & & & & \\
\hline 2613.96 & 6 & & $(0.177)$ & 1.132 & & & & \\
\hline 2607.10 & 4 & 0.209 & $(0.106)$ & 1.347 & $21 / 2$ & $1 / 2$ & 1.656 & 1.864 \\
\hline 2606.00 & 5 & 0.137 & $(0.068)$ & 1.666 & $41 / 2$ & $31 / 2$ & 1.187 & 1.050 \\
\hline 2591.547 & 6 & 0.224 & $(0.565)$ & & $21 / 2$ & $21 / 2$ & & \\
\hline 2579.437 & 4 & 0.320 & $(0.160)$ & 0.807 & $1 / 2$ & $1 / 2$ & 0.967 & 1.287 \\
\hline 2563.477 & 4 or 5 & 0.362 & $(0.186)$ & & $21 / 2$ & $1 / 2$ & & \\
\hline 2562.530 & 4 or 5 & 0.159 & $(0.075)$ & & $31 / 2$ & $21 / 2$ & & \\
\hline 2560.94 & 4 & 0.192 & $(0.091)$ & 0.277 & $4 / 2$ & $31 / 2$ & 0.949 & 1.141 \\
\hline 2549.98 & 6 & 0.186 & $(0.651)$ & & $31 / 2$ & $31 / 2$ & & \\
\hline 2537.764 & 6 & 0.228 & $(0.566)$ & & $21 / 2$ & $21 / 2$ & & \\
\hline 2517.948 & 4 or 5 & 0.362 & $(0.181)$ & & $31 / 2$ & $2 \frac{1}{2}$ & & \\
\hline 2494.64 & 6 & 0.189 & $(0.663)$ & 1.303 & $31 / 2$ & $31 / 2$ & 1.398 & 1.209 \\
\hline 2485.976 & 5 & 0.644 & $(0.322)$ & 2.122 & $2 \frac{1}{2}$ & $11 / 2$ & 1.156 & 0.512 \\
\hline 2481.40 & 6 & 0.471 & $(1.178)$ & & $21 / 2$ & $21 / 2$ & & \\
\hline 2470.591 & 4 & 0.153 & $(0.074)$ & 0.734 & $2 \frac{1}{2}$ & $11 / 2$ & 0.964 & 1.117 \\
\hline 2450.81 & 4 & 0.145 & $(0.072)$ & 0.405 & $4 \frac{1}{2}$ & $31 / 2$ & 0.913 & 1.058 \\
\hline 2443.88 & 5 & 0.367 & $(0.176)$ & 1.727 & $21 / 2$ & $11 / 2$ & 1.177 & 0.810 \\
\hline 2436.34 & 6 & & $(0.147)$ & 1.275 & & & & \\
\hline 2404.895 & 4 or 5 & 0.254 & $(0.126)$ & & $31 / 2$ & $21 / 2$ & & \\
\hline 2368.96 & 4 or 5 & 0.264 & $(0.139)$ & & $3 \% 2$ & $2 \frac{1}{2}$ & & \\
\hline 2362.11 & 6 & & $(0.294)$ & 0.563 & & & & \\
\hline 2344.31 & 6 & & $(0.443)$ & 2.721 & & & & \\
\hline 2338.03 & 6 & & $(0.802)$ & 1.823 & & & & \\
\hline 2330.87 & 6 & & $(0.117)$ & 0.644 & & & & \\
\hline
\end{tabular}




\section{THORIUM}

\section{Th I}

90 Electrons

Ground State: $1 s^{2} 2 s^{2} 2 p^{6} 3 s^{2} 3 p^{6} 3 d^{10} 4 s^{2} 4 p^{6} 4 d^{10} 4 f^{14} 5 s^{2} 5 p^{6} 5 d^{10} 6 s^{2} 6 p^{6} 6 d^{2} 7 s^{2}{ }^{3} \mathrm{~F}_{2}$

The analysis of this spectrum is quite incomplete. The levels are taken from the dissertation by Schuurmans and an unpublished list of McNally. More work is needed on all phases of this spectrum.

\section{REFERENCES}

Ph. Schuurmans, Doctoral Dissertation, Amsterdam (1946). (T)

J. N. Lier, Doctoral Dissertation, Amsterdam (1939). (ZE)

J. R. McNally, Jr., unpublished material (1950). (T)

G. L. Stukenbroeker and J. R. McNally, Jr., J. Opt. Soc. Am. 43, 36 (1953). (IS) 
Table 8. Energy Levels of Th I

\begin{tabular}{|c|c|c|c|c|c|}
\hline Configuration & Designation & $J$ & $\begin{array}{l}\text { Level } \\
\left(\mathrm{cm}^{-1}\right)\end{array}$ & Observed $g$ Value & $\begin{array}{c}\sigma_{232}-\sigma_{230} \\
\left(\mathrm{~cm}^{-1}\right)\end{array}$ \\
\hline $6 d^{2} 7 s^{2}{ }^{3} \mathrm{~F}_{2}$ & 1 & 2 & 0.00 & 0.738 & 0.54 \\
\hline $6 d^{2} 7 s^{2}{ }^{3} F_{3}$ & 2 & 3 & 2869.24 & 1.086 & 0.49 \\
\hline $6 d^{2} 7 s^{2}{ }^{3} \mathrm{~F}_{4} ?$ & 3 & 4 & 5093.55 & 1.18 & \\
\hline $6 d^{3} 7 s^{5} \mathrm{~F}_{1} ?$ & 4 & 1 & 5563.18 & 0.067 & \\
\hline \multirow[t]{29}{*}{$6 d^{3} 7 s^{5} F_{2} ?$} & 5 & 2 & 6362.39 & 1.008 & \\
\hline & 6 & 3 & 7502.25 & 1.155 & \\
\hline & 7 & 2,3 & 8545.50 & & \\
\hline & 8 & 0 & 8605.88 & & \\
\hline & 9 & 1 & 8662.29 & $1.49 ?$ & \\
\hline & 10 & 1 & 10806.72 & $1.574 ?$ & \\
\hline & 11 & 4 & 12083.41 & $0.997 ?$ & \\
\hline & 12 & 4 & 13297.42 & 0.997 & \\
\hline & $13^{\circ}$ & 1 & 15396.27 & 0.508 & \\
\hline & $14^{\circ}$ & 1 & 17354.64 & 0.55 & \\
\hline & $15^{\circ}$ & 4 & 19511.76 & 1.305 & \\
\hline & $16^{\circ}$ & $2 ?$ & 19570.87 & & \\
\hline & $17^{\circ}$ & 1,2 & 20423.57 & $1.42^{a}$ & \\
\hline & $19^{\circ}$ & 1,2 & 21252.61 & $0.68^{a}$ & \\
\hline & $20^{\circ}$ & 1 & 21668.94 & 1.571 & \\
\hline & $21^{\circ}$ & 2,3 & 22141.55 & $1.10^{a}$ & \\
\hline & $22^{\circ}$ & 2 & 22248.92 & 1.140 & \\
\hline & $23^{\circ}$ & 2 & 22508.02 & $1.38^{a}$ & \\
\hline & $24^{\circ}$ & 1 & 23481.38 & 0.845 & \\
\hline & $25^{\circ}$ & 2 & 23603.52 & 1.381 & \\
\hline & $26^{\circ}$ & 2 & 24624.00 & 1.252 & \\
\hline & $27^{\circ}$ & 3 & 24769.70 & 1.138 & 0.00 \\
\hline & $28^{\circ}$ & 2 & 25051.01 & 1.131 & \\
\hline & $29^{\circ}$ & 4 & 25059.54 & 1.321 & \\
\hline & $30^{\circ}$ & $3 ?$ & 25401.65 & $0.502 ?$ & \\
\hline & $31^{\circ}$ & $?$ & $25834.91 ?$ & $0.999 ?$ & \\
\hline & $32^{\circ}$ & 3 & 26048.69 & $1.055 ?$ & 0.06 \\
\hline & $33^{\circ}$ & 2 & 26113.27 & 0.985 & 0.14 \\
\hline & $34^{\circ}$ & 2 & $26287.06 ?$ & 0.74 & \\
\hline
\end{tabular}

\footnotetext{
${ }^{a}$ Values taken from Schuurmans.
} 
Table 8 (continued)

\begin{tabular}{|c|c|c|c|c|c|}
\hline Configuration & Designation & $J$ & $\begin{array}{l}\text { Level } \\
\left(\mathrm{cm}^{-1}\right)\end{array}$ & Observed $g$ Value & $\begin{array}{c}\sigma_{232}-\sigma_{230} \\
\left(\mathrm{~cm}^{-1}\right)\end{array}$ \\
\hline & $35^{\circ}$ & 3 & 26508.01 & 1.080 & 0.06 \\
\hline & $36^{\circ}$ & 3 & 26878.17 & 0.900 & 0.17 \\
\hline & $37^{\circ}$ & 2 & 27447.78 & 1.067 & 0.04 \\
\hline & \multirow[t]{2}{*}{$38^{\circ}$} & 3 & 27670.93 & 1.215 & 0.10 \\
\hline & & & & $1.32^{a}$ & \\
\hline & $39^{\circ}$ & 2 & 27674.36 & 1.103 & 0.10 \\
\hline & $40^{\circ}$ & 2 & 27784.34 & 0.863 & 0.06 \\
\hline & $41^{\circ}$ & $3 ?$ & 27789.06 & $1.09 ?$ & \\
\hline & $42^{\circ}$ & $3 ?$ & 27806.03 & $1.04 ?$ & \\
\hline & $43^{\circ}$ & 1 & 28024.70 & 1.030 & 0.05 \\
\hline & $44^{\circ}$ & $4 ?$ & $28666.13 ?$ & $1.07 ?$ & 0.01 \\
\hline & $45^{\circ}$ & $4 ?$ & 28676.29 & 1.063 & 0.11 \\
\hline & $46^{\circ}$ & $3 ?$ & 28884.96 & 1.192 & 0.10 \\
\hline & $47^{\circ}$ & 2 & 28917.90 & $0.98^{a}$ & 0.00 \\
\hline & $49^{\circ}$ & $1 ?$ & 29197.32 & $1.14^{a}$ & \\
\hline & $50^{\circ}$ & $4 ?$ & $29806.91 ?$ & & \\
\hline & $51^{\circ}$ & 2 & 29851.46 & 0.901 & \\
\hline & $52^{\circ}$ & 2 & 29853.17 & $0.90^{a}$ & 0.06 \\
\hline & $53^{\circ}$ & 3 & 30017.12 & 1.128 & 0.11 \\
\hline & $55^{\circ}$ & 3 & 30255.44 & 1.079 & \\
\hline & $56^{\circ}$ & 1 & $31756.63 ?$ & $0.494^{b}$ & \\
\hline & $57^{\circ}$ & $3 ?$ & $32090.40 ?$ & $1.08^{a}$ & \\
\hline & $58^{\circ}$ & 2 & 32356.98 & 0.963 & \\
\hline & $59^{\circ}$ & 2 & 32954.64 & $1.04^{a}$ & \\
\hline
\end{tabular}

$a_{\text {Values taken from Schuurmans. }}$

$b_{\text {Th III?. }}$ 
Table 9. Classified Lines of Th I

\begin{tabular}{|c|c|c|c|c|c|c|c|c|c|c|c|c|}
\hline \multirow{2}{*}{ Ref } & \multirow{2}{*}{$\lambda(\stackrel{\circ}{A})$} & \multirow{2}{*}{$\begin{array}{c}\sigma_{\text {vac }} \\
\left(\mathrm{cm}^{-1}\right)\end{array}$} & \multirow{2}{*}{$\begin{array}{l}\Delta \sigma_{\text {vac }} \\
\left(\mathrm{cm}^{-1}\right)\end{array}$} & \multirow{2}{*}{ Transition } & \multirow{2}{*}{ Ref } & \multicolumn{5}{|c|}{ Zeeman Effect (Lorentz units) } & \multirow{2}{*}{$\begin{array}{c}\sigma_{230}-\sigma_{232} \\
\left(\mathrm{~cm}^{-1}\right)\end{array}$} & \multirow{2}{*}{ Notes } \\
\hline & & & & & & Type & $p$ & $n$ & $g_{1}$ & $g_{2}$ & & \\
\hline$c^{a}$ & 8478.36 & 11791.50 & +0.04 & $14^{\circ}-4$ & & & & & & & & \\
\hline$M^{b}$ & 7567.72 & 13210.39 & -0.20 & $35^{\circ}-12$ & & & & & & & & \\
\hline c & 7361.40 & 13580.64 & -0.11 & $36^{\circ}-12$ & & & & & & & & \\
\hline C & 7353.06 & 13596.04 & -0.01 & $21^{\circ}-7$ & & & & & & & & \\
\hline$M$ & 6829.05 & 14639.30 & 0.00 & $21^{\circ}-6$ & & & & & & & & \\
\hline$M$ & 6727.48 & 14860.31 & -0.08 & $17^{\circ}-4$ & & & & & & & & Th II? \\
\hline$c$ & 6714.01 & 14890.13 & -0.09 & $19^{\circ}-5$ & & & & & & & & \\
\hline$M$ & 6662.274 & 15005.75 & -0.02 & $23^{\circ}-6$ & & & & & & & & \\
\hline$M$ & 6531.348 & 15306.55 & 0.00 & $20^{\circ}-5$ & $M$ & 1 & $(0.000)$ & 0.445 & 1.571 & 1.008 & & \\
\hline$M$ & 6493.29 & 15396.27 & 0.00 & $13^{\circ}-1$ & $M$ & 2 & $(0.000)$ & 0.968 & 0.508 & 0.738 & & \\
\hline \multirow[t]{2}{*}{$M$} & 6413.612 & 15587.54 & 0.00 & $46^{\circ}-12$ & $M$ & 1 & $(0.000)$ & 0.419 & 1.192 & 0.997 & & \\
\hline & & & +0.02 & $38^{\circ}-11$ & & & & & & & & \\
\hline$M$ & 6371.936 & 15689.49 & +0.06 & $19^{\circ}-4$ & & & & & & & & \\
\hline$M$ & 6207.218 & 16105.58 & -0.18 & $20^{\circ}-4$ & & & & & & & & \\
\hline$M$ & 6191.895 & 16145.69 & +0.06 & $23^{\circ}-5$ & & & & & & & & Th $\| ?$ \\
\hline$M$ & 6079.15 & 16645.13 & 0.00 & $28^{\circ}-8$ & & & & & & & & \\
\hline$M$ & 6007.068 & 16642.65 & +0.13 & $15^{\circ}-2$ & $M$ & 2 & $(0.000)$ & 1.960 & 1.305 & 1.087 & & \\
\hline$M$ & 5950.18 & 16801.57 & +0.02 & $46^{\circ} \cdot 11$ & & & & & & & & \\
\hline$c$ & 5899.83 & 16944.96 & +0.12 & $23^{\circ}-4$ & & & & & & & & \\
\hline $\mathrm{K}^{c}$ & 5895.29 & 16958.01 & -0.01 & $55^{\circ}-12$ & & & & & & & & Th $\| ?$ \\
\hline$M$ & 5838.951 & 17121.62 & -0.13 & $26^{\circ}-6$ & & & & & & & & Th $\|$ ? \\
\hline$M$ & 5806.26 & 17218.03 & +0.05 & $43^{\circ}-10$ & & & & & & & & \\
\hline$C$ & 5798.45 & 17241.22 & +0.09 & $25^{\circ} \cdot 5$ & & & & & & & & \\
\hline$M$ & 5789.60 & 17267.57 & +0.12 & $27^{\circ}-6$ & & & & & & & & \\
\hline$c$ & 5782.26 & 17289.49 & +0.08 & $31^{\circ}-7$ & & & & & & & & \\
\hline$M$ & 5760.554 & 17354.63 & +0.01 & $14^{\circ}-1$ & $\mathrm{~L}^{d}$ & & $(0)$ & 0.90 & 0.61 & 0.80 & & \\
\hline$K$ & 5640.703 & 17723.38 & -0.12 & $50^{\circ}-11 ?$ & & & & & & & & Th III? \\
\hline
\end{tabular}

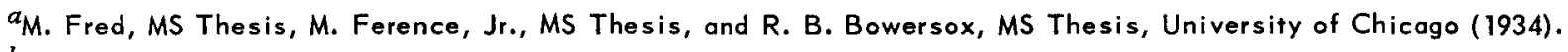

${ }^{b}$ MIT data are taken from either MIT wavelength tables or MIT card files on thorium. MIT Zeeman data are also taken from J. R. MeNally, Jr., G. R. Harrison, and H. B. Park, J. Opt. Soc. Am. 32, 334 (1942).

${ }^{c}$ P. F. A. Klinkenberg, unpublished wavelength list and unpublished Zeeman data.

dj. N. Lier, Doctoral Dissertation, Amsterdam (1939). 
Table 9 (continued)

\begin{tabular}{|c|c|c|c|c|c|c|c|c|c|c|c|c|}
\hline \multirow{2}{*}{ Ref } & \multirow{2}{*}{$\lambda(\stackrel{\circ}{\AA})$} & \multirow{2}{*}{$\begin{array}{c}\sigma_{v a c} \\
\left(\mathrm{~cm}^{-1}\right)\end{array}$} & \multirow{2}{*}{$\begin{array}{l}\Delta \sigma_{\mathrm{vac}} \\
\left(\mathrm{cm}^{-1}\right)\end{array}$} & \multirow{2}{*}{ Transition } & \multirow{2}{*}{ Ref } & \multicolumn{5}{|c|}{ Zeeman Effect (Lorentz units) } & \multirow{2}{*}{$\begin{array}{c}\sigma_{230}-\sigma_{232} \\
\left(\mathrm{~cm}^{-1}\right)\end{array}$} & \multirow{2}{*}{ Notes } \\
\hline & & & & & & Type & $p$ & $n$ & $g_{1}$ & $8_{2}$ & & \\
\hline$M$ & 5579.371 & 17918.21 & +0.01 & $24^{\circ} \cdot 4$ & & & & & & & & \\
\hline C & 5541.58 & 18040.40 & +0.06 & $25^{\circ}-4$ & & & & & & & & \\
\hline$M$ & 5431.123 & 18407.29 & -0.02 & $27^{\circ} \cdot 5$ & & & & & & & & Th $\|$ ? \\
\hline$c$ & 5390.40 & 18546.36 & -0.08 & $32^{\circ} \cdot 6$ & & & & & & & & \\
\hline$M$ & 5260.11 & 19005.74 & -0.02 & $35^{\circ}-6$ & & & & & & & & \\
\hline$M$ & 5258.358 & 19012.08 & +0.01 & $39^{\circ}-9$ & & & & & & & & \\
\hline $\mathrm{C}$ & 5250.87 & 19039.18 & -0.08 & $30^{\circ}-5$ & & & & & & & & \\
\hline$c$ & 5187.31 & 19272.46 & +0.15 & $21^{\circ}-2$ & & & & & & & & \\
\hline K & 5159.58 & 19376.04 & +0.12 & $36^{\circ}-6$ & & & & & & & & \\
\hline$M$ & 5158.607 & 19379.70 & +0.02 & $22^{\circ}-2$ & & & & & & & & \\
\hline$M$ & 5148.215 & 19418.82 & 0.00 & $43^{\circ}-8$ & $\mathrm{~K}$ & & $(0)$ & 1.03 & & & & Th II? \\
\hline$M$ & 5108.25 & 19570.74 & -0.13 & $16^{\circ}-1$ & $L$ & & $(0)$ & 0.74 & & & & Th 111 ? \\
\hline C & 5090.56 & 19638.75 & -0.03 & $23^{\circ}-2$ & & & & & & & & \\
\hline$c$ & 5078.25 & 19686.35 & +0.05 & $32^{\circ}-5$ & & & & & & & & \\
\hline$M$ & 5061.657 & 19750.90 & +0.02 & $33^{\circ} .5$ & & & & & & & & \\
\hline$c$ & 4956.8 & 20168.7 & 0.00 & $38^{\circ}-6$ & & & & & & & & \\
\hline$M$ & 4955.98 & 20172.0 & -0.1 & $39^{\circ}-6$ & & & & & & & & \\
\hline$M$ & 4929.081 & 20282.1 & 0.0 & $40^{\circ}-6$ & & & & & & & & Th $\|$ ? \\
\hline M & 4907.238 & 20372.39 & -0.01 & $47^{\circ} \cdot 7$ & & & & & & & & \\
\hline$M$ & 4894.954 & 20423.52 & -0.05 & $17^{\circ} \cdot 1$ & & & & & & & & \\
\hline$M$ & 4872.926 & 20515.84 & +0.06 & $36^{\circ}-5$ & & & & & & & & \\
\hline$M$ & 4840.826 & 20651.89 & +0.07 & $49^{\circ}-7$ & & & & & & & & \\
\hline$M$ & 4824.029 & 20723.90 & +0.02 & $34^{\circ} \cdot 4$ & & & & & & & & Th II? \\
\hline$M$ & 4821.589 & 20734.27 & -0.01 & $25^{\circ}-2$ & & & & & & & & \\
\hline C & 4771.94 & 20950.0 & +0.1 & $56^{\circ} \cdot 10$ & & & & & & & & \\
\hline$M$ & 4770.77 & 20955.1 & 0.0 & $32^{\circ}-3$ & & & & & & & & Th $\|$ ? \\
\hline$M$ & 4741.32 & 21085.3 & -0.1 & $37^{\circ}-5$ & & & & & & & & \\
\hline$M$ & 4723.683 & 21164.02 & +0.14 & $44^{\circ}-6$ & $M$ & 1 & & 0.787 & 1.063 & 1.155 & & \\
\hline$M$ & 4715.436 & 21201.03 & 0.00 & $50^{\circ}-8$ & & & & & & & & Th II? \\
\hline$c$ & 4702.08 & 21261.3 & -0.1 & $50^{\circ} \cdot 7$ & & & & & & & & \\
\hline$M$ & 4691.628 & 21308.62 & +0.08 & $38^{\circ}-5$ & & & & & & & & \\
\hline$K$ & 4675.33 & 21382.9 & +0.2 & $46^{\circ}-6$ & & & & & & & & \\
\hline$M$ & 4668.20 & 21415.6 & -0.1 & $47^{\circ}-6$ & $M$ & & $(0.000)$ & $\Delta g=0.296$ & & & & \\
\hline$M$ & 4666.804 & 21421.96 & +0.01 & $40^{\circ} \cdot 5$ & & & & & & & & \\
\hline
\end{tabular}


Table 9 (continued)

\begin{tabular}{|c|c|c|c|c|c|c|c|c|c|c|c|c|}
\hline \multirow{2}{*}{ Ref } & \multirow{2}{*}{$\lambda(\stackrel{\circ}{)}$} & \multirow{2}{*}{$\begin{array}{c}\sigma_{\text {vac }} \\
\left(\mathrm{cm}^{-1}\right)\end{array}$} & \multirow{2}{*}{$\begin{array}{l}\Delta \sigma_{\text {vac }} \\
\left(\mathrm{cm}^{-1}\right)\end{array}$} & \multirow{2}{*}{ Transition } & \multirow{2}{*}{ Ref } & \multicolumn{5}{|c|}{ Zeeman Effect (Lorentz units) } & \multirow{2}{*}{$\begin{array}{c}\sigma_{230}-\sigma_{232} \\
\left(\mathrm{~cm}^{-1}\right)\end{array}$} & \multirow{2}{*}{ Notes } \\
\hline & & & & & & Type & $p$ & $n$ & $g_{1}$ & $g_{2}$ & & \\
\hline C & 4615.0 & 21662.4 & +0.1 & $43^{\circ}-5$ & & & & & & & & \\
\hline$M$ & 4595.424 & 21754.70 & +0.14 & $26^{\circ}-2$ & $M$ & 1 & $(0.000)$ & 0.763 & 1.152 & 1.088 & & \\
\hline$M$ & 4589.119 & 21784.59 & -0.03 & $36^{\circ}-3$ & $L$ & 3 & $(1.18)$ & $0.89,1.18$ & 0.89 & 1.18 & & \\
\hline$M$ & 4568.142 & 21884.62 & +0.02 & $37^{\circ}-4$ & & & & & & & & \\
\hline $\mathrm{C}$ & 4564.86 & 21900.4 & -0.1 & $27^{\circ}-2$ & & & & & & & & \\
\hline$M$ & 4515.124 & 22141.60 & +0.05 & $21^{\circ}-1$ & & & & & & & & Th $\| ?$ \\
\hline M & 4505.226 & 22190.24 & -0.06 & $29^{\circ}-2$ & M & 2 & & 1.948 & 1.321 & 1.112 & & \\
\hline$M$ & 4498.950 & 22221.20 & +0.04 & $40^{\circ}-4$ & $M$ & 2 & $(0.000)$ & 1.645 & 0.861 & 0.077 & & \\
\hline$M$ & 4493.340 & 22248.94 & +0.02 & $22^{\circ} \cdot 1$ & $M$ & 3 & $(0.812)$ & $1.140,0.733$ & 1.140 & 0.733 & & \\
\hline$c$ & 4480.26 & 22313.9 & 0.0 & $45^{\circ}-5$ & & & & & & & & \\
\hline c & 4473.20 & 22349.1 & -0.1 & $51^{\circ}-6$ & & & & & & & & \\
\hline$M$ & 4450.787 & 22461.65 & +0.13 & $43^{\circ} \cdot 4$ & & & & & & & & \\
\hline$c$ & 4441.60 & 22508.1 & +0.1 & $23^{\circ}-1$ & & & & & & & & \\
\hline$M$ & 4438.750 & 22522.56 & -0.01 & $46^{\circ}-5$ & & & & & & & & \\
\hline$M$ & 4432.262 & 22555.53 & +0.02 & $47^{\circ}-5$ & & & & & & & & \\
\hline$M$ & 4410.383 & 22667.42 & +0.12 & $54^{\circ}-6$ & & & & & & & & \\
\hline$M$ & 4404.929 & 22695.49 & -0.02 & $41^{\circ}-3$ & & & & & & & & Th II? \\
\hline $\mathrm{C}$ & 4401.59 & 22712.7 & +0.2 & $42^{\circ}-3$ & & & & & & & & \\
\hline C & 4393.75 & 22753.2 & 0.0 & $55^{\circ} \cdot 6$ & & & & & & & & \\
\hline C & 4328.88 & 23094.2 & -0.1 & $56^{\circ}-9$ & & & & & & & & \\
\hline$v$ & 4327.26 & 23102.8 & -0.1 & $44^{\circ}-4$ & & & & & & & & \\
\hline$M$ & 4318300 & 23150.77 & +0.02 & $56^{\circ}-8$ & & & & & & & & Th II? \\
\hline c & 4312.97 & 23179.4 & -0.1 & $32^{\circ}-2$ & & & & & & & 0.43 & \\
\hline$v$ & 4280.57 & 23354.8 & +0.1 & $47^{\circ}-4$ & & & & & & & & \\
\hline$M$ & 4257.502 & 23481.37 & -0.01 & $24^{\circ}-1$ & $M$ & 1 & $(0.000)$ & 0.625 & 0.845 & 0.735 & & \\
\hline$M$ & 4256.102 & 23489.09 & +0.02 & $51^{\circ} \cdot 5$ & & & & & & & & Th $11 ?$ \\
\hline$M$ & 4255.789 & 23490.82 & +0.04 & $52^{\circ}-5$ & & & & & & & & \\
\hline$M$ & 4235.468 & 23603.52 & 0.00 & $25^{\circ}-1$ & $M$ & 3 & $(1.283)$ & $1.381,0.740$ & 1.381 & 0.740 & & \\
\hline V & 4229.17 & 23638.7 & -0.1 & $35^{\circ}-2$ & & & & & & & & \\
\hline
\end{tabular}

e Vatican data from A. Gatterer and J. Junkes, Spektren der Seltenen Erden, p 329-347, Specola Vaticana, Vatican City, 1945. 
Table 9 (continued)

\begin{tabular}{|c|c|c|c|c|c|c|c|c|c|c|c|c|}
\hline \multirow{2}{*}{ Ref } & \multirow{2}{*}{$\lambda(\stackrel{\circ}{\AA})$} & \multirow{2}{*}{$\begin{array}{c}\sigma_{\mathrm{rac}} \\
\left(\mathrm{cm}^{-1}\right)\end{array}$} & \multirow{2}{*}{$\begin{array}{c}\Delta \sigma_{\text {vac }} \\
\left(\mathrm{cm}^{-1}\right)\end{array}$} & \multirow{2}{*}{ Transition } & \multirow{2}{*}{ Ref } & \multicolumn{5}{|c|}{ Zeeman Effect (Lorentz units) } & \multirow{2}{*}{$\begin{array}{c}\sigma_{230}-\sigma_{232} \\
\left(\mathrm{~cm}^{-1}\right)\end{array}$} & \multirow{2}{*}{ Notes } \\
\hline & & & & & & Type & $p$ & $n$ & $g_{1}$ & $g_{2}$ & & \\
\hline $\mathrm{C}$ & 4184.12 & 23983.2 & +0.1 & $55^{\circ}-5$ & & & & & & & & \\
\hline $\mathrm{C}$ & 4163.93 & 24009.0 & +0.1 & $36^{\circ}-2$ & & & & & & & & \\
\hline M & 4115.773 & 24289.94 & -0.05 & $52^{\circ}-4$ & & & & & & & & \\
\hline$M$ & 4067.465 & 24578.42 & -0.12 & $37^{\circ}-2$ & & & & & & & & \\
\hline$M$ & 4036.056 & 24769.69 & -0.01 & $27^{\circ}-1$ & $\mathrm{~L}$ & & $(0.00)$ & 1.98 & 1.190 & 0.792 & 0.54 & Th $\|{ }^{2}$ \\
\hline$M$ & 4030.855 & 24801.65 & -0.04 & $38^{\circ}-2$ & $M$ & 3 & $(0.384)$ & $1.216,1.085$ & 1.215 & 1.085 & 0.39 & \\
\hline$M$ & 4030.293 & 24805.11 & -0.01 & $39^{\circ}-2$ & & & & & & & & Th $\|$ ? \\
\hline$M$ & 4012.497 & 24915.12 & +0.02 & $40^{\circ}-2$ & $M$ & 2 & $(0.000)$ & 1.525 & 0.864 & 1.086 & 0.46 & Th $\| ?$ \\
\hline$M$ & 4011.752 & 24919.75 & -0.07 & $41^{\circ}-2$ & $\mathrm{~L}$ & & $(0)$ & 1.09 & & & & Th $\| ?$ \\
\hline$M$ & 4009.066 & 24936.44 & -0.35 & $42^{\circ}-2$ & $L$ & & $(0.08)$ & 1.04 & & & & \\
\hline$K$ & 3990.730 & 25051.00 & -0.01 & $28^{\circ} \cdot 1$ & $L$ & & $(0.81)$ & $0.71,1.14$ & 1.14 & 0.71 & & Th $\|1\|$ P \\
\hline$M$ & 3935.642 & 25401.65 & 0.00 & $30^{\circ}-1$ & & & & & & & & Th II? \\
\hline C & 3927.82 & 25452.2 & -0.2 & $59^{\circ} \cdot 6$ & & & & & & & & \\
\hline$M$ & 3875.379 & 25796.64 & -0.25 & $44^{\circ}-2$ & $L$ & & $(0)$ & 1.02 & & & 0.38 & \\
\hline$M$ & 3873.823 & 25807.00 & -0.05 & $45^{\circ}-2$ & $L$ & & $(0)$ & 1.07 & & & 0.48 & \\
\hline$M$ & 3869.639 & 25834.91 & 0.00 & $31^{\circ}-1$ & & & & & & & & \\
\hline \multirow[t]{2}{*}{$M$} & 3837.880 & 26048.69 & +0.03 & $47^{\circ}-2$ & $M$ & 2 & $(0.000)$ & 1.425 & 1.021 & 1.156 & 0.49 & Th $\|$ ? \\
\hline & & & 0.00 & $32^{\circ}-1$ & & & & & & & & \\
\hline$M$ & 3828.388 & 26113.27 & 0.00 & $33^{\circ}-1$ & $M$ & 3 & $(0.499)$ & $0.985,0.735$ & 0.985 & 0.735 & 0.40 & \\
\hline$M$ & 3803.077 & 26287.06 & 0.00 & $34^{\circ}-1$ & $K$ & & $(0)$ & 0.74 & & & & Th $\| ?$ \\
\hline$M$ & 3771.377 & 26508.01 & 0.00 & $35^{\circ}-1$ & $M$ & 2 & $(0.000)$ & 1.760 & 1.080 & 0.740 & 0.48 & \\
\hline$M$ & 3759.445 & 26592.14 & -0.11 & $59^{\circ} \cdot 5$ & & & & & & & & \\
\hline$M$ & 3719.436 & 26878.18 & +0.01 & $36^{\circ} \cdot 1$ & $M$ & 2 & $(0.000)$ & 1.221 & 0.900 & 0.738 & 0.37 & \\
\hline v & 3704.86 & 26984.2 & +0.3 & $52^{\circ}-2$ & & & & & & & & \\
\hline$M$ & 3682.490 & 27147.84 & -0.04 & $53^{\circ}-2$ & & & & & & & & \\
\hline$M$ & 3649.737 & 27391.46 & 0.00 & $59^{\circ}-4$ & & & & & & & & \\
\hline$M$ & 3642.249 & 27445.89 & +0.11 & $37^{\circ}-1$ & $M$ & 3 & $(0.669)$ & $1.067,0.730$ & 1.067 & 0.730 & 0.50 & \\
\hline$M$ & 3612.869 & 27670.97 & +0.04 & $38^{\circ}-1$ & & & & & & & & \\
\hline$M$ & 3612.427 & 27674.36 & 0.00 & $39^{\circ}-1$ & $M$ & 3 & $(0.714)$ & $1.103,0.733$ & 1.103 & 0.733 & 0.44 & \\
\hline$M$ & 3598.124 & 27784.36 & +0.02 & $40^{\circ}-1$ & $M$ & 3 & $(0.248)$ & $0.857,0.738$ & 0.863 & 0.738 & 0.48 & Th $\|$ ? \\
\hline$M$ & 3597.498 & 27789.20 & +0.14 & $41^{\circ}-1$ & & & & & & & & \\
\hline$M$ & 3595.323 & 27806.01 & -0.02 & $42^{\circ}-1$ & & & & & & & & \\
\hline$M$ & 3567.266 & 28024.70 & 0.00 & $43^{\circ} \cdot 1$ & $M$ & 1 & $(0.000)$ & 0.438 & 1.030 & 0.735 & 0.49 & \\
\hline
\end{tabular}


Table 9 (continued)

\begin{tabular}{|c|c|c|c|c|c|c|c|c|c|c|c|c|}
\hline \multirow{2}{*}{ Rof } & \multirow{2}{*}{$\lambda(\stackrel{\AA}{A})$} & \multirow{2}{*}{$\begin{array}{c}\sigma_{\text {vac }} \\
\left(\mathrm{cm}^{-1}\right)\end{array}$} & \multirow{2}{*}{$\begin{array}{l}\Delta \sigma_{\text {vac }} \\
\left(\mathrm{cm}^{-1}\right)\end{array}$} & \multirow{2}{*}{ Transition } & \multirow{2}{*}{ Rof } & \multicolumn{5}{|c|}{ Zeeman Effect (Lorentz units) } & \multirow{2}{*}{$\begin{array}{c}\sigma_{230}-\sigma_{232} \\
\left(\mathrm{~cm}^{-1}\right)\end{array}$} & \multirow{2}{*}{ Notes } \\
\hline & & & & & & Type & $p$ & $n$ & $g_{1}$ & $g_{2}$ & & \\
\hline c & 3486.20 & 28676.3 & 0.0 & 1 & & & & & & & & \\
\hline M & 3461.021 & 28884.97 & +0.01 & $46^{\circ}-1$ & M & 2 & $(0.000)$ & 2.096 & 1.192 & 0.739 & 0.44 & \\
\hline M & 3457.075 & 28917.93 & +0.03 & $47^{\circ}-1$ & & & & & & & & \\
\hline M & 3427.03 & 29171.5 & 0.0 & $48^{\circ}-1$ & & & & & & & & \\
\hline M & 3423.991 & 29197.34 & +0.02 & $49^{\circ} \cdot 1$ & & & & & & & & \\
\hline M & 3421.189 & 29221.25 & +0.09 & $57^{\circ} \cdot 2$ & & & & & & & & \\
\hline M & 3353.96 & 29807.0 & +0.1 & $50^{\circ}-1$ & & & & & & & & Th $\|$ ? \\
\hline M & 3348.961 & 29851.46 & 0.00 & $51^{\circ} \cdot 1$ & M & 3 & $(0.320)$ & $0.901,0.725$ & 0.901 & 0.725 & & \\
\hline M & 3348.766 & 29853.20 & +0.03 & $52^{\circ} \cdot 1$ & & & & & & & 0.48 & \\
\hline M & 3330.475 & 30017.18 & +0.06 & $53^{\circ}-1$ & M & 2 & $(0.000)$ & 1.909 & 1.128 & 0.739 & 0.43 & \\
\hline c & 3313.67 & 30169.4 & -0.2 & $54^{\circ}-1$ & M & 2 & $(0.000)$ & 1.366 & 0.914 & 0.744 & & Th III? \\
\hline M & 3304.239 & 30255.48 & +0.04 & $55^{\circ}-1$ & M & 2 & $(0.000)$ & 1.770 & 1.079 & 0.731 & 0.46 & \\
\hline M & 3148.040 & 31756.60 & -0.03 & $56^{\circ}-1$ & M & 2 & $(0.000)$ & 1.059 & 0.494 & 0.769 & & Th $\| 1$ ? \\
\hline M & 3089.631 & 32356.96 & -0.02 & $58^{\circ}-1$ & M & 3 & $(0.416)$ & & 0.963 & 0.767 & & $\begin{array}{l}\text { Th } \| l ? \\
\text { Th } \| 11 ?\end{array}$ \\
\hline
\end{tabular}


Table 10. Zeeman Data of Unclassified Th I Lines

\begin{tabular}{|c|c|c|c|c|c|c|c|c|c|}
\hline$\lambda(\stackrel{\circ}{(\mathrm{A})}$ & Type & $\begin{array}{c}\text { Separation } \\
\text { (Lorentz units) }\end{array}$ & $\begin{array}{c}p \\
\text { (Lorentz units) }\end{array}$ & $\begin{array}{c}n \\
\text { (Lorentz units) }\end{array}$ & $J_{1}$ & $J_{2}$ & $g_{1}$ & $g_{2}$ & Notes \\
\hline 6470.62 & 3 & 0.140 & $(0.266)$ & & 2 & 2 & & & \\
\hline 6457.285 & 2 & 0.141 & $(0.000)$ & & 5 & 4 & 1.006 & 0.865 & \\
\hline 6449.44 & 2 & 0.382 & $(0.000)$ & 1.293 & 2 & 1 & 0.908 & 0.526 & \\
\hline 6411.893 & 1 & 0.071 & $(0.000)$ & 1.101 & 3 & 2 & 1.243 & 1.314 & \\
\hline 6358.629 & 1 & & $(0.000)$ & 0.715 & 5 & 4 & & & \\
\hline 6342.861 & 2 & 0.118 & $(0.000)$ & 1.666 & 4 & 3 & 1.312 & 1.194 & \\
\hline 6077.547 & 1 or 2 & 0.313 & & & 3 & 2 & & & \\
\hline 5437.519 & 1 or 2 & 0.190 & & & 4 & 3 & & & \\
\hline 5382.927 & 1 & 0.182 & $(0.000)$ & 0.538 & 3 & 2 & 0.898 & 1.080 & \\
\hline 4943.57 & 1 & 0.29 & $(0.00)$ & 0.89 & 2 & 1 & 1.19 & 1.49 & \\
\hline 4822.758 & 1 or 2 & & & & 2 & 1 & & & \\
\hline 4677.06 & 3 & 0.117 & $(0.852)$ & & 4 & 4 & & & \\
\hline 4662.800 & 1 & 0.143 & $(0.000)$ & 0.425 & 2 & $\mathfrak{l}$ & 0.568 & 0.711 & \\
\hline 4649.397 & 3 & 0.127 & & & 3 & 3 & & & \\
\hline 4634.130 & 1 or 2 & 0.197 & & & 2 & 1 & & & \\
\hline 4555.742 & 3 & 0.221 & $(0.438)$ & $1.128,0.907$ & 2 & 2 & 1.128 & 0.907 & Th III? \\
\hline 4535.257 & 1 or 2 & 0.157 & & & 2 & 1 & & & \\
\hline 4531.721 & 3 & 0.155 & $(0.472)$ & & 3 & 3 & & & \\
\hline 4469.528 & 3 & 0.100 & $(0.399)$ & & 4 & 4 & & & \\
\hline 4458.006 & 3 & 0.261 & $(0.530)$ & & 2 & 2 & & & \\
\hline 4408.891 & 3 & 0.190 & $(0.439)$ & & 2 & 2 & & & \\
\hline 4399.180 & 3 & 0.165 & & & 3 & 3 & & & \\
\hline 4378.127 & 1 or 2 & 0.060 & & & 3 & 2 & & & \\
\hline 4365.909 & 2 & 0.313 & $(0.000)$ & 1.719 & 3 & 2 & 1.093 & 0.780 & \\
\hline 4346.450 & 1 or 2 & 0.277 & $(0.000)$ & & 2 & 1 & & & \\
\hline 4299.846 & 1 or 2 & 0.065 & $(0.000)$ & & 4 & 3 & & & \\
\hline 4296.95 & 3 & 0.125 & $(0.632)$ & & 5 & 5 & & & \\
\hline 4291.812 & 1 or 2 & 0.112 & $(0.000)$ & & 3 & 2 & & & \\
\hline 4278.323 & 3 & 0.085 & $(0.425)$ & & 5 & 5 & & & \\
\hline 4272.885 & 1 or 2 & 0.166 & $(0.000)$ & & 4 & 3 & & & \\
\hline 4270.332 & 1 or 2 & 0.335 & $(0.000)$ & & 3 & 2 & & & \\
\hline 4260.341 & 2 & 0.192 & $(0.000)$ & 2.139 & 5 & 4 & 1.371 & 1.179 & \\
\hline 4227.421 & 1 or 2 & 0.205 & $(0.000)$ & & 2 & 1 & & & \\
\hline 4170.570 & 3 & 0.120 & $(0.411)$ & & 4 & 4 & & & \\
\hline 4165.813 & 3 & 0.228 & $(0.913)$ & & 4 & 4 & & & \\
\hline 4112.762 & 3 & 0.774 & & 1.549 & 2 & 2 & & & \\
\hline 4102.662 & 1 or 2 & 0.301 & $(0.000)$ & & 3 & 2 & & & \\
\hline 4059.259 & 2 & 0.082 & $(0.000)$ & 1.261 & 3 & 2 & 1.096 & 1.014 & \\
\hline 4053.531 & 3 & & $(1.103)$ & & 4 & 4 & 1.003 & 0.735 & \\
\hline 4022.068 & 1 or 2 & 0.550 & $(0.000)$ & & 3 & 2 & & & \\
\hline 4008.216 & 1 or 2 & 0.272 & $(0.000)$ & & 4 & 3 & & & \\
\hline 3980.884 & 3 & 0.121 & & & 2 & 2 & 1.230 & 1.109 & \\
\hline 3967.426 & 3 & 0.096 & $(0.381)$ & & 4 & 4 & & & \\
\hline 3895.42 & 3 & 0.077 & $(0.327)$ & & 4 & 4 & 0.903 & 0.826 & \\
\hline 3852.154 & 2 & 0.295 & $(0.000)$ & 1.560 & 2 & 1 & 1.254 & 0.959 & \\
\hline 3790.902 & 1 or 2 & 0.188 & $(0.000)$ & & 4 & 3 & & & \\
\hline
\end{tabular}


Table 10 (continued)

\begin{tabular}{|c|c|c|c|c|c|c|c|c|c|}
\hline$\lambda(\stackrel{\circ}{\mathrm{A}})$ & Type & $\begin{array}{l}\text { Separation } \\
\text { (Lorentz units) }\end{array}$ & $\begin{array}{c}p \\
\text { (Lorentz units) }\end{array}$ & $\begin{array}{c}n \\
\text { (Lorentz units) }\end{array}$ & $J_{1}$ & $J_{2}$ & $g_{1}$ & $g_{2}$ & Notes \\
\hline 3790.851 & 1 or 2 & 0.168 & $(0.000)$ & & 3 & 2 & & & \\
\hline \multirow[t]{2}{*}{3788.35} & 1 or 2 & 0.36 & $(0.00)$ & 1.91 & 3 & 2 & 1.19 or 2.63 & 0.83 & \\
\hline & & & & & & & & 2.99 & \\
\hline 3780.97 & 2 & 0.21 & $(0.00)$ & 2.44 & 3 & 2 & 2.02 & 1.81 & \\
\hline 3770.09 & 3 & 0.734 & $(0.734)$ & & 1 & 1 & 0.791 & 0.057 & \\
\hline 3754.113 & 1 or 2 & 0.314 & $(0.000)$ & & 3 & 2 & & & \\
\hline 3733.074 & 3 & 0.140 & $(0.410)$ & & 3 & 3 & & & \\
\hline 3730.470 & 1 or 2 & 0.331 & $(0.000)$ & & 2 & 1 & & & \\
\hline 3727.991 & 3 & 0.181 & $(0.534)$ & 1.259 & 3 & 3 & 1.261 & 1.080 & \\
\hline 3711.31 & 2 & 0.19 & $(0.00)$ & 1.62 & 4 & 3 & 1.05 & 0.86 & \\
\hline 3708.851 & 3 & 0.123 & & & 4 & 4 & & & \\
\hline 3700.410 & 3 & 0.213 & $(0.855)$ & & 4 & 4 & & & \\
\hline 3698.204 & 1 or 2 & 0.934 & $(0.000)$ & & 2 & 1 & & & \\
\hline 3668.147 & 1 & 0.115 & $(0.000)$ & 0.660 & 2 & 1 & 0.776 & 0.891 & \\
\hline 3667.095 & 3 & 0.208 & $(0.838)$ & & 4 & 4 & & & \\
\hline 3655.490 & 3 & 0.160 & $(0.803)$ & & 5 & 5 & & & \\
\hline 3652.272 & 3 & 0.117 & $(0.666)$ & & 5 & 5 & 1.059 & 1.176 & \\
\hline 3643.611 & 1 or 2 & 0.092 & $(0.000)$ & & 3 & 2 & & & \\
\hline 3638.85 & 3 & 0.191 & $(0.570)$ & & 3 & 3 & & & \\
\hline 3608.445 & 1 or 2 & 0.235 & $(0.000)$ & & 4 & 3 & & & \\
\hline 3591.455 & 1 & 0.160 & $(0.000)$ & 0.789 & 3 & 2 & 1.109 & 1.269 & \\
\hline 3584.186 & 3 & 0.108 & $(0.319)$ & & 3 & 3 & & & \\
\hline 3565.39 & 2 & 0.201 & $(0.000)$ & 1.803 & 5 & 4 & 1.000 & 0.799 & Th II? \\
\hline 3561.809 & 2 & 0.200 & $(0.000)$ & 1.601 & 3 & 2 & 1.207 & 1.005 & \\
\hline 3544.014 & 1 & 0.174 & $(0.000)$ & 0.420 & 5 & 4 & 1.116 & 1.290 & \\
\hline 3422.677 & 3 & 0.202 & $(0.402)$ & & 2 & 2 & & & \\
\hline 3415.905 & 3 & 0.153 & $(0.305)$ & & 2 & 2 & & & \\
\hline 3405.574 & 2 & 0.146 & $(0.000)$ & & 3 & 2 & & & \\
\hline 3387.939 & 3 & 0.160 & $(0.631)$ & & 4 & 4 & & & \\
\hline 3195.691 & 1 or 2 & 0.380 & $(0.000)$ & & 3 & 2 & & & \\
\hline 3173.553 & 1 or 2 & 0.209 & $(0.000)$ & & 3 & 2 & & & \\
\hline 3171.281 & 1 or 2 & 0.362 & $(0.000)$ & & 3 & 2 & & & \\
\hline 3145.672 & 1 or 2 & 0.440 & $(0.000)$ & & 3 & 2 & & & \\
\hline 3136.864 & 3 & 0.206 & $(0.412)$ & & 2 & 2 & & & \\
\hline 3124.42 & 3 & 0.30 & $(0.95)$ & $0.64,0.96$ & 3 & 3 & 0.96 & 0.64 & \\
\hline 3069.251 & 1 & & $(0.000)$ & 0.963 & 3 & 2 & & & \\
\hline 3063.026 & 3 & 0.171 & $(0.169)$ & 1.207 & 1 & 1 & 1.207 & 1.378 & Th $\|$ ? \\
\hline 3049.866 & 3 & 0.140 & $(0.457)$ & & 3 & 3 & 1.369 & 1.229 & Th II? \\
\hline 3041.553 & 1 or 2 & 0.185 & $(0.000)$ & & 2 & 1 & & & \\
\hline 3013.649 & 2 & 0.783 & $(0.000)$ & 1.432 & 2 & 1 & 1.246 & 1.063 & \\
\hline 2827.992 & 1 & 0.274 & $(0.000)$ & 0.342 & 3 & 2 & 0.908 & 1.182 & Th $\|$ ? \\
\hline 2787.639 & 1 or 2 & 0.142 & $(0.000)$ & & 4 & 3 & & & \\
\hline 2630.017 & 1 & & $(0.000)$ & 0.776 & 4 & 3 & & & \\
\hline 2614.447 & 1 or 2 & 0.384 & $(0.000)$ & & 2 & 1 & & & \\
\hline \multirow[t]{2}{*}{2442.68} & 1 or 2 & 0.116 & $(0.000)$ & 1.127 & 3 & 2 & 0.895 or 1.359 & 0.779 & \\
\hline & & & & & & & & 1.475 & \\
\hline 2333.730 & 1 or 2 & 0.286 & $(0.000)$ & & 2 & 1 & & & \\
\hline
\end{tabular}


Table 11. Isotope Shift Data of Unclassified Lines

\begin{tabular}{|c|c|c|c|c|}
\hline \multirow{2}{*}{$\lambda \stackrel{\circ}{(\mathrm{A})}$} & \multicolumn{2}{|c|}{ Intensity } & \multirow{2}{*}{$\begin{array}{c}\sigma_{230}-\sigma_{232} \\
\left(\mathrm{~cm}^{-1}\right)\end{array}$} & \multirow{2}{*}{ Probable Spectrum } \\
\hline & Arc & Spark & & \\
\hline 4673.661 & 6 & 2 & 0.6 & Th 1 \\
\hline 4644.371 & 3 & & 0.42 & Th 1 \\
\hline 4408.891 & 6 & 2 & 0.6 & Th I (Zeeman Effect) \\
\hline 4307.185 & 5 & 3 & 0.7 & Th II \\
\hline 4306.381 & 8 & 6 & 0.7 & Th II \\
\hline 4270.332 & 6 & 3 & 0.40 & Th I (Zeeman Effect) \\
\hline 4240.59 & 2 & & 0.6 & Th I \\
\hline 4230.431 & 5 & 1 & 0.4 & Th I \\
\hline 4193.022 & 2 & & 0.5 & Th I \\
\hline 4132.751 & 10 & 12 & 0.38 & Th II \\
\hline 4112.762 & 5 & 5 & 0.40 & Th II (I-Zeeman Effect) \\
\hline 4100.350 & 8 & 5 & 0.4 & Th $\|$ \\
\hline 4005.095 & 3 & 1 & 0.43 & Th I \\
\hline 3990.558 & 8 & 5 & 0.5 & Th 11 \\
\hline 3988.601 & 10 & 8 & 0.6 & Th $\|$ \\
\hline 3972.159 & 15 & 8 & 0.35 & Th 1 \\
\hline 3895.425 & 20 & 10 & 0.39 & Th I (Zeeman Effect) \\
\hline 3874.866 & 5 & 3 & 0.47 & Th 1 \\
\hline 3866.09 & & 2 & 0.6 & Th II \\
\hline 3852.135 & 5 & & 0.5 & Th I (Zeeman Effect) \\
\hline 3742.925 & 20 & 8 & 0.5 & Th 1 \\
\hline 3737.513 & 5 & 1 & 0.5 & ThI \\
\hline 3730.383 & 15 & 3 & 0.45 & Th I (Zeeman Effect ?) \\
\hline 3727.903 & 15 & 5 & 0.4 & Th 1 \\
\hline 3724.739 & 30 & 20 & 0.4 & Th $\|$ \\
\hline 3712.538 & 15 & 8 & 0.5 & Th 1 \\
\hline 3692.571 & 10 & 2 & 0.4 & Th 1 \\
\hline 3669.958 & 5 & 1 & 0.6 & Th I \\
\hline 3635.944 & 8 & 4 & 0.38 & Th 1 \\
\hline 3634.584 & 8 & 4 & 0.40 & Th 1 \\
\hline 3601.040 & 8 & 10 & 0.4 & Th II \\
\hline 3598.786 & & 2 & 0.75 & Th $\|$ \\
\hline 3547.339 & $2 d$ & ld & 0.7 & Th II \\
\hline 3531.451 & 4 & 1 & 0.6 & Th I \\
\hline 3495.703 & 10 & 1 & 0.5 & Th I \\
\hline 3477.705 & 8 & 8 & 0.5 & Th $\|$ \\
\hline 3469.341 & 6 & 3 & 0.42 & Th I \\
\hline 3466.647 & 5 & 2 & 0.4 & Th I \\
\hline 3466.536 & 5 & 1 & 0.5 & Th I \\
\hline 3461.218 & 10 & 4 & 0.56 & Th 1 \\
\hline 3442.581 & 5 & 1 & 0.7 & Th I \\
\hline 3437.310 & 6 & 2 & 0.49 & Th 1 \\
\hline 3429.000 & 6 & 3 & 0.5 & Th I \\
\hline 3210.273 & 10 & 10 & 0.42 & Th $\|$ \\
\hline 3139.822 & 10 & 5 & 0.36 & Th I \\
\hline
\end{tabular}


Table 11 (continued)

\begin{tabular}{ccccc}
\hline \multirow{2}{*}{\begin{tabular}{c} 
(A) $)$ \\
\cline { 2 - 5 }
\end{tabular}} & Arc & Spark & $\begin{array}{c}\sigma_{230}-\sigma_{232} \\
(\mathrm{~cm}-1)\end{array}$ & Probable Spectrum \\
\hline 3100.939 & 12 & 2 & 0.50 & Th I \\
3067.734 & 12 & 20 & 0.45 & Th II \\
2999.093 & 12 & 12 & 0.40 & Th II \\
2925.050 & $10 \mathrm{~s}$ & $15 \mathrm{~h}$ & 0.45 & Th II \\
\hline
\end{tabular}


Table 12. Unclassified Furnace Lines of Thorium

\begin{tabular}{|c|c|c|c|c|}
\hline \multirow{2}{*}{$\lambda(\stackrel{\circ}{(\mathrm{A})}$} & \multicolumn{2}{|c|}{ Intensity } & \multirow{2}{*}{ Notes } & \multirow{2}{*}{ Probable Spectrum } \\
\hline & Arc & Spark & & \\
\hline 5044.72 & 8 & & & Th I \\
\hline 4954.665 & 10 & 2 & II $152^{\circ}-167 ?$ & Th I \\
\hline 4878.739 & 8 & & & Th I \\
\hline 4848.355 & 8 & & & Th I \\
\hline 4831.15 & 10 & 2 & & Th I \\
\hline 4826.40 & 1 & & $1176^{\circ}-18 ?$ & Th I \\
\hline 4809.625 & 2 & & & Th I \\
\hline 4808.127 & 2 & 1 & & \\
\hline 4789.381 & 2 & & II $136^{\circ}-147 ?$ & \\
\hline 4778.287 & 2 & & & \\
\hline 4766.595 & 2 & & & \\
\hline 4563.316 & 2 & & & \\
\hline 4563.228 & 5 & 5 & & Th $\|$ \\
\hline 4537.11 & 20 & 8 & II $135^{\circ}-169 ?$ & Th I \\
\hline 4499.96 & 12 & 6 & & \\
\hline 4493.31 & 12 & 4 & & \\
\hline 4482.170 & 5 & & & \\
\hline 4458.009 & 10 & 2 & II Zeeman Effect? & Th I \\
\hline 4408.886 & 6 & 2 & I Zeeman Effect & Th I \\
\hline 4378.183 & 2 & 1 & I Zeeman Effect? & \\
\hline 4374.139 & 4 & 1 & & \\
\hline 4313.003 & 8 & 4 & & Th I \\
\hline 4210.928 & 8 & 3 & & Th 1 \\
\hline 4210.768 & 3 & 1 & & Th 1 \\
\hline 4112.762 & 5 & $5 w$ & I Zeeman Effect & Th 1 \\
\hline 4097.751 & 5 & & & \\
\hline 3967.412 & 2 & 1 & I Zeeman Effect & Th 1 \\
\hline 3895.424 & 20 & 10 & & ? \\
\hline 3886.918 & 8 & 1 & & Th 1 \\
\hline 3659.632 & 4 & 1 & & Th I \\
\hline 3658.808 & 5 & 1 & & Th 1 \\
\hline 3526.629 & 5 & 1 & & Th I \\
\hline 3521.062 & $3 d$ & Id & & \\
\hline 3518.402 & 6 & 2 & & Th 1 \\
\hline 3511.154 & 5 & & & Th 1 \\
\hline 3498.625 & 6 & 3 & II Zeeman Effect & \\
\hline 3496.809 & 5 & 3 & & \\
\hline 3495.705 & 10 & 1 & & Th 1 \\
\hline 3488.837 & 3 & & & Th 1 \\
\hline 3471.219 & 6 & 2 & & Th I \\
\hline 3469.337 & 6 & 3 & & Th 1 \\
\hline 3466.646 & 5 & 2 & & Th 1 \\
\hline 3466.532 & 5 & 1 & & Th I \\
\hline 3461.216 & 10 & 4 & & Th 1 \\
\hline 3451.699 & 8 & 5 & & Th 1 \\
\hline
\end{tabular}


Table 12 (continued)

\begin{tabular}{|c|c|c|c|c|}
\hline \multirow{2}{*}{$\lambda(\stackrel{\circ}{A})$} & \multicolumn{2}{|c|}{ Intensity } & \multirow{2}{*}{ Notes } & \multirow{2}{*}{ Probable Spectrum } \\
\hline & Arc & Spark & & \\
\hline 3442.580 & 5 & 1 & & Th 1 \\
\hline 3437.303 & 6 & 2 & & Th 1 \\
\hline 3428.990 & 6 & 3 & & Th I \\
\hline \multirow[t]{2}{*}{3422.657} & 3 & 1 & $1119^{\circ}-39 ?$ & Th 1 \\
\hline & & & I Zeeman Effect? & \\
\hline 3417.494 & 3 & & & Th 1 \\
\hline 3413.015 & 5 & 2 & II $149^{\circ}-164$ ? & Th I \\
\hline 3408.748 & 5 & 1 & & Th I \\
\hline 3405.560 & 3 & 4 & I Zeeman Effect & Th I \\
\hline 3398.548 & 5 & 2 & II Zeeman Effect & Th I \\
\hline 3397.518 & 8 & 4 & & Th I \\
\hline 3396.733 & 5 & 1 & & Th I \\
\hline 3389.464 & 3 & & & Th I \\
\hline 3380.883 & 5 & 2 & & Th I \\
\hline 3374.979 & 3 & 3 & & Th II \\
\hline 3373.500 & 2 & 1 & II Zeeman Effect & Th II \\
\hline 3372.794 & $3 d$ & $4 d$ & & Th II \\
\hline 3372.696 & ld & $2 d$ & & \\
\hline 3333.129 & 6 & 2 & & Th 1 \\
\hline 3309.361 & 4 & 1 & & Th I \\
\hline 3301.654 & 6 & $1 \mathrm{~h}$ & $1155^{\circ}-121 ?$ & Th I \\
\hline 3298.054 & 4 & $1 \mathrm{~h}$ & & Th I \\
\hline 3285.750 & 8 & 1 & & Th I \\
\hline 3272.025 & 6 & 1 & & Th 1 \\
\hline 3257.35 & 6 & 2 & & Th I \\
\hline 3253.872 & 5 & 1 & & Th I \\
\hline 3244.450 & $5 d$ & ld & & Th I \\
\hline 3232.307 & 8 & & & \\
\hline 3232.123 & 3 & $25 \mathrm{~h}$ & $+111 ?$ & \\
\hline
\end{tabular}


Table 13. Unclassified Absorption Lines of Thorium

\begin{tabular}{|c|c|c|c|c|}
\hline \multirow{2}{*}{$\lambda \stackrel{\circ}{(\mathrm{A})}$} & \multicolumn{2}{|c|}{ Intensity } & \multirow{2}{*}{ Degree of Absorption } & \multirow{2}{*}{ Probable Spectrum } \\
\hline & Arc & Spark & & \\
\hline 5028.61 & $40 w h$ & 10wh & 3 & Th I \\
\hline 4987.17 & 15 & 4 & 5 & Th 1 \\
\hline 4563.23 & 5 & 5 & 3 & Th $\|$ \\
\hline 4256.23 & 6 & 1 & 5 & Th I \\
\hline 4253.50 & 8 & 3 & 5 & Th I \\
\hline 4171.35 & 10 & 121 & 5 & Th II Zeeman Effect \\
\hline 4168.62 & 8 & 12 & 5 & Th II \\
\hline 4057.51 & & & 5 & \\
\hline 3895.42 & 20 & 10 & 5 & Th I or II Zeeman Effect \\
\hline 3874.85 & 5 & 3 & 5 & Th $\|$ \\
\hline 3839.69 & 15 & 15 & 3 & Th H \\
\hline 3828.43 & 5 & 5 & 5 & Th 11 \\
\hline 3804.70 & 10 & 8 & 3 & Th $\|$ \\
\hline 3720.30 & 15 & 10 & 1 & Th $11 *$ \\
\hline 3711.32 & 30 & 20 & 3 & Th I Zeeman Effect \\
\hline 3700.80 & 15 & 10 & 3 & Th 11 \\
\hline 3693.95 & 2 & 3 & 3 & Th II Zeeman Effect \\
\hline 3669.94 & $5 d$ & ld & 5 & Th 1 \\
\hline 3659.63 & 4 & 1 & 3 & Th 1 \\
\hline 3634.584 & 8 & 4 & 5 & Th II \\
\hline 3617.038 & $4 d$ & $3 d$ & 5 & Th II \\
\hline 3601.040 & 8 & 10 & 2 & Th $\|$ \\
\hline 3589.299 & 5 & 5 & 3 & Th $\|$ \\
\hline 3553.111 & 5 & 6 & 4 & Th $\|$ Zeemon Effect \\
\hline 3511.615 & 10 & 8 & 1 & Th II \\
\hline 3421.189 & 10 & 10 & 5 & Th II + I? Zeeman Effect (II) \\
\hline 3408.750 & 5 & 1 & 3 & Th 1 \\
\hline 3398.548 & 5 & 2 & 5 & Th I II? Zeeman Elfect (II) \\
\hline 3397.87 & & 2 & 5 & Th II \\
\hline 3397.519 & 8 & 4 & 5 & Th I \\
\hline 3389.464 & 3 & & 4 & Th I \\
\hline 3382.36 & & & 5 & \\
\hline 3320.81 & & loh & 5 & Th $\|$ or $\| I$ \\
\hline 3301.347 & $6 d$ & $5 d$ & 2 & Th 11 \\
\hline 3257.369 & 6 & 2 & 3 & Th 1 \\
\hline 3225.409 & & & 3 & \\
\hline 3108.36 & & & 3 & \\
\hline 3082.031 & 12 & 8 & 5 & Th II \\
\hline 3072.18 & & & 3 & \\
\hline 3067.734 & 12 & 20 & 3 & Th II \\
\hline 2999.093 & 12 & 12 & 3 & Th II \\
\hline 2985.31 & & & 4 & \\
\hline 2983.86 & & & 4 & \\
\hline
\end{tabular}

* The line has been classified, but the absorption seems too strong to be consistent with the classification. 
Table 13 (continued)

\begin{tabular}{|c|c|c|c|c|}
\hline \multirow{2}{*}{$\lambda(\stackrel{\circ}{(\mathrm{A})}$} & \multicolumn{2}{|c|}{ Intensity } & \multirow{2}{*}{ Degree of Absorption } & \multirow{2}{*}{ Probable Spectrum } \\
\hline & Arc & Spark & & \\
\hline 2981.56 & & & 3 & \\
\hline 2958.26 & & & 4 & \\
\hline 2957.98 & & & 3 & \\
\hline 2924.99 & & & 3 & \\
\hline 2891.819 & 6 & 5 & 3 & Th II \\
\hline 2891.31 & & & 3 & \\
\hline 2885.10 & & & 3 & \\
\hline 2814.318 & 8 & 5 & 4 & Th $\|$ \\
\hline 2809.09 & & & 3 & \\
\hline 2786.917 & 5 & 8 & 3 & Th II \\
\hline 2786.16 & & & 5 & \\
\hline 2785.56 & & & 5 & \\
\hline 2784.92 & & & 5 & \\
\hline 2783.99 & & & 5 & \\
\hline 2783.44 & & & 5 & \\
\hline 2777.86 & & & 3 & \\
\hline 2762.05 & & & 5 & \\
\hline 2759.415 & 4 & 3 & 5 & Th II \\
\hline 2749.63 & 2 & & 3 & Th \| \\
\hline 2738.46 & & & 5 & \\
\hline 2731.583 & 5 & 4 & 5 & Th II Zeeman Effect \\
\hline 2726.43 & & & 5 & \\
\hline 2723.41 & & & 5 & \\
\hline 2715.092 & 6 & 5 & 5 & Th II Zeeman Effect \\
\hline 2714.617 & $2 \mathrm{~h}$ & & 5 & Th $\|$ \\
\hline 2708.08 & & & 3 & \\
\hline 2701.86 & $2 \mathrm{~h}$ & & 5 & Th II Zeeman Effect \\
\hline 2695.555 & 8 & 10 & 5 & Th II \\
\hline 2672.88 & & & 5 & \\
\hline 2671.44 & & & 5 & Th II Zeeman Effect \\
\hline 2669.43 & & & 5 & Th II Zeeman Effect \\
\hline 2664.02 & & & 5 & \\
\hline 2662.866 & 8 & 5 & 5 & Th II \\
\hline 2659.47 & & & 5 & \\
\hline 2652.02 & & & 5 & \\
\hline 2643.40 & & & 5 & \\
\hline 2642.615 & 5 & 2 & 5 & Th II Zeeman Effect \\
\hline 2640.33 & $5 d$ & $2 d$ & 5 & Th II \\
\hline 2638.32 & & & 5 & \\
\hline 2628.39 & & & 5 & Th II Zeeman Effect \\
\hline 2560.94 & 6 & 2 & 5 & Th II Zeeman Effect \\
\hline 2509.84 & & & 5 & \\
\hline 2502.903 & 6 & 3 & 5 & \\
\hline 2494.57 & & & 5 & Th II Zeeman Effect \\
\hline 2470.597 & 6 & 4 & 3 & Th II Zeeman Effect \\
\hline 2456.86 & 4 & 2 & 5 & Th II \\
\hline
\end{tabular}


Table 13 (continued)

\begin{tabular}{|c|c|c|c|c|}
\hline \multirow{2}{*}{$\lambda \stackrel{\circ}{(\mathrm{A})}$} & \multicolumn{2}{|c|}{ Intensity } & \multirow{2}{*}{ Degree of Absorption } & \multirow{2}{*}{ Probable Spectrum } \\
\hline & Are & Spork & & \\
\hline 2450.81 & 5 & 3 & 5 & Th II Zeeman Effect \\
\hline 2437.56 & 5 & 4 & 3 & Th $\|$ \\
\hline 2423.675 & 6 & 5 & 1 & Th II \\
\hline 2422.99 & 1 & 1 & 4 & Th $\|$ \\
\hline 2411.25 & & & 3 & \\
\hline 2407.51 & & & 4 & \\
\hline 2407.13 & 1 & 1 & 4 & Th $\|$ \\
\hline 2406.68 & 1 & 1 & 4 & Th \| \\
\hline 2395.62 & 3 & 1 & 2 & Th $\|$ \\
\hline 2385.55 & & & 4 & \\
\hline 2384.36 & 1 & 2 & 4 & Th $\|$ \\
\hline 2375.835 & 5 & 3 & 4 & Th $\|$ \\
\hline 2368.88 & & & 2 & Th II Zeeman Effect \\
\hline 2366.984 & 3 & 5 & 2 & Th $\|^{*}$ \\
\hline 2359.95 & & & 5 & \\
\hline 2330.94 & & & 2 & Th \| Zeeman Effect \\
\hline 2308.58 & 1 & 1 & 5 & Th $\|$ \\
\hline 2306.00 & 1 & ih & 5 & \\
\hline 2305.52 & & & 5 & \\
\hline 2281.98 & 1 & $2 \mathrm{~h}$ & 3 & Th II \\
\hline 2265.90 & 1 & 1 & 3 & Th II \\
\hline 2244.89 & & & 5 & \\
\hline 2242.55 & & & 5 & \\
\hline 2240.33 & & & 3 & \\
\hline 2224.30 & & & 5 & \\
\hline 2221.09 & & & 3 & \\
\hline 2200.24 & & & 5 & \\
\hline 2198.70 & & & 5 & \\
\hline
\end{tabular}

* The line has been classified, but the absorption seems too strong to be consistent with the classification. 
-

- 
ORNL-2319

Physics and Mathematics

TID-4500 (13th ed., Rev.)

INTERNAL DISTRIBUTION

1. C. E. Center

2. Biology Library

3. Health Physics Library

4-5. Central Research Library

6. Reactor Experimental Engineering Library

7-26. Laboratory Records Department

27. Laboratory Records, ORNL R.C.

28. A. M. Weinberg

29. L. B. Emlet (K-25)

30. J. P. Murray (Y-12)

31. J. A. Swartout

32. E. H. Taylor

33. E. D. Shipley

34. A. H. Snell

35. M. L. Nel son

36. W. H. Jordan

37. G. E. Boyd

38. R. A. Charpie

39. S. C. Lind

40. F. L. Culler

41. A. Hollaender

42. J. H. Frye, Jr.

43. W. M. Good

44. M. T. Kelley

45. J. L. Fowler

46. R. S. Livingston

47. K. Z. Morgan

48. T. A. Lincoln

49. A. S. Householder

50. C. P. Keim

51. C. S. Harrill

52. C. E. Winters

53. D. S. Billington

54. D. W. Cardwell

55. D. Phillips

56. E. O. Wollan

57. A. J. Miller

58. J. A. Lane
59. P. M. Reyling

60. L. D. Roberts

61. R. N. Lyon

62. W. C. Koehler

63. E. P. Blizard

64. M. E. Rose

65. M. J. Skinner

66. J. E. Sherwood

67. R. B. Murray

68. R. R. Dickison

69. J. A. Harvey

70. A. Simon

71. J. J. Pinajian

72. F. C. Maien schein

73. J. R. McNally

74. P. M. Griffin

75. K. L. Vander Sluis

76. J. A. Norris

77. M. R. Skidmore

78. G. K. Werner

79. W. F. Peed

80. O. B. Rudolph

81. P. A. Staats

82. H. W. Morgan

83. S. J. Ovenshine

84. C. Feldman

85. T. Lee

86. M. Murray

87. L. E. Burkhart

88-107. G. W. Charles

108. W. R. Brode (consultant)

109. R. F. Christy (consultant)

110. M. Goldhaber (consultant)

111. T. Lauritsen (consultant)

112. N. F. Ramsey (consultant)

113. J. A. Wheeler (consultant)

114-115. ORNL - Y-12 Technical Library,

Document Reference Section

EXTERNAL DISTRIBUTION

116. Division of Research and Development, AEC, ORO

117. W. K. H. Panofsky, Stanford University

118. A. Glassgold, University of Minnesota 
119-121. NBS, Spectroscopy Section, Washington (1 copy ea. to W. F. Meggers, C. C. Kiess, and C. M. Sitterly)

122-123. Argonne National Laboratory (1 copy ea. to M. Fred and F. S. Tompkins)

124. G. L. Stukenbroeker, National Lead Company of Ohio, Mt. Healthy Station, Cincinnati

125. G. R. Harrison, Massachusetts Institute of Technology

126. J. G. Conway, University of California Radiation Laboratory

127-730. Given distribution as shown in TID-4500 (13th ed., Rev.) under Physics and Mathematics category ( 75 copies - OTS) 